\title{
FY96-98 Summary Report Mercury: Next Generation Laser for High Energy Density Physics SI-014
}

A. Bayramian, R. Beach, C. Bibeau, J.-C. Chanteloup, C. Ebbers, M. Emanuel, B. Freitas, S. Fulkerson, K. Kanz, A. Hinz, C. Marshall, S. Mills, H. Nakano, C. Orth, J. Rothenberg, K. Schaffers, L. Seppala, J. Skidmore, L. Smith, S. Sutton, S. Telford, L. Zapata

\section{May 23, 2000}

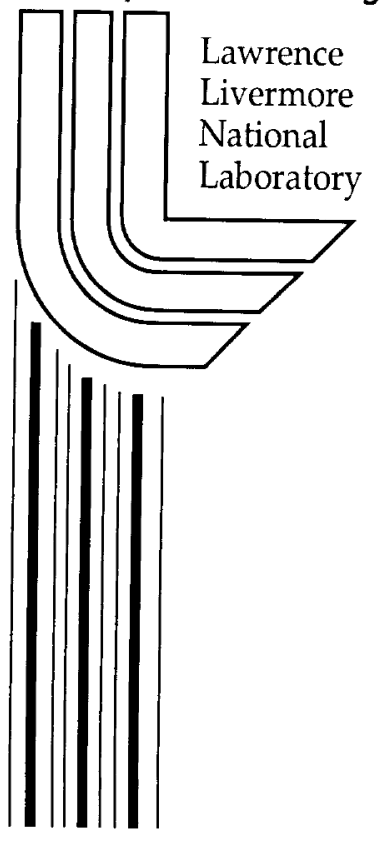




\section{DISCLAIMER}

This document was prepared as an account of work sponsored by an agency of the United States Government. Neither the United States Government nor the University of California nor any of their employees, makes any warranty, express or implied, or assumes any legal liability or responsibility for the accuracy, completeness, or usefulness of any information, apparatus, product, or process disclosed, or represents that its use would not infringe privately owned rights. Reference herein to any specific commercial product, process, or service by trade name, trademark, manufacturer, or otherwise, does not necessarily constitute or imply its endorsement, recommendation, or favoring by the United States Government or the University of California. The views and opinions of authors expressed herein do not necessarily state or reflect those of the United States Government or the University of California, and shall not be used for advertising or product endorsement purposes.

This work was performed under the auspices of the U. S. Department of Energy by the University of California, Lawrence Livermore National Laboratory under Contract No. W-7405-Eng-48.

This report has been reproduced directly from the best available copy.

Available electronically at http://www.doe.gov/bridge

Available for a processing fee to U.S. Department of Energy

and its contractors in paper from

U.S. Department of Energy

Office of Scientific and Technical Information

$$
\text { P.O. Box } 62
$$

Oak Ridge, TN 37831-0062

Telephone: (865) 576-8401

Facsimile: (865) 576-5728

E-mail: reports@adonis.osti.gov

Available for the sale to the public from

U.S. Department of Commerce

National Technical Information Service 5285 Port Royal Road Springfield, VA 22161

Telephone: (800) 553-6847

Facsimile: (703) 605-6900

E-mail: orders@ntis.fedworld.gov

Online ordering: http://www.ntis.gov/ordering.htm

OR

Lawrence Livermore National Laboratory

Technical Information Department's Digital Library

http://www.llnl.gov/tid/Library.html 


\section{FY96-98 Summary Report Mercury: Next Generation Laser for High Energy Density Physics SI-014}
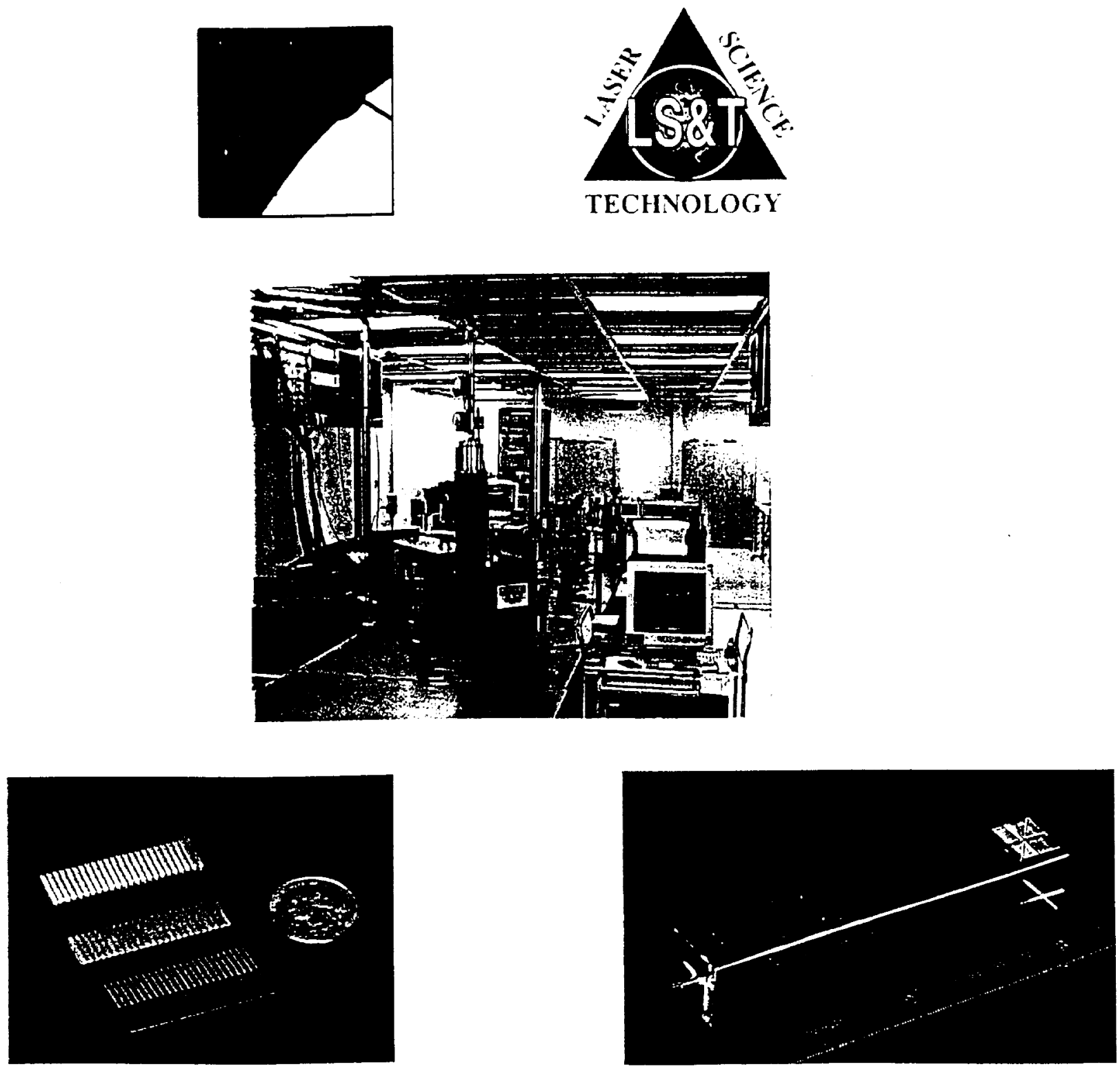

CAUTION: DO NOT PUBLICLY RELEASE THIS DOCUMENT

This technical report is being transmitted prior to DOE patent clearance. No further dissemination or publication shall be made without prior approval of DOE Patent Counsel or LLNL Patent Group. This document will be returned upon request or when no longer needed, unless notification has been received that this document has been cleared for release or publication 


\section{FY96-98 Summary Report Mercury: Next Generation Laser for High Energy Density Physics SI-014}

Contributors: Andy Bayramian, Raymond Beach, Camille Bibeau (P.I.), Jean-Christophe Chanteloup, Christopher Ebbers, Mark Emanuel, Barry Freitas, Steve Fulkerson, Keith Kanz, Andrew Hinz, Chris Marshall, Steve Mills, Hitoshi Nakano, Charles Orth, Josh Rothenberg, Kathleen Schaffers, Lynn Seppala, Jay Skidmore, Larry Smith, Steve Sutton, Steve Telford, and Luis Zapata

Laser Science and Technology Program Program Leader: Howard Powell Associate Program Leader: Steve Payne Lawrence Livermore National Laboratory Livermore, California 94550

May 23, 2000

Revision 1.0 


\section{Table of Contents}

1. Introduction and Motivation for Building the Mercury Laser

2. Conceptual Layout

3. Laser Requirements

4. Major Architectural Elements
A. Gas Cooled Amplitier Head
B. Diode Pump Delivery
C. System Layout

5. Czochralski Crystal Growth
A. Growth techniques
B. Wavefront analysis, bonding technique

6. Laser Diodes
A. $900 \mathrm{~nm}$ Diode Bars
B. Heatsinks
C. Microlensing
D. Backplane Cooler
E. Future Directions

7. Diode Power Conditioning

8. Computer Controls

9. System Utilities

10. System Modeling
A. System Energetics
B. Beam Smoothing Options
C. Thermal Wavefront Distortions
D. Propagation Modeling with the Prop 92 Code

11. Integration Experiments

A. Gain Measurements with Surrogate Nd:Glass Amplifier Media

B. Thermal Wavefront Measurements

C. Measurements of the Temperature Distribution in the Lens Duct and Homogenizer

12. Summary 


\section{Scope of Report}

The scope of the Mercury Laser project encompasses the research. development. and engineering required to build a new generation of diode-pumped solid-state lasers for Inertial Confinement Fusion (ICF). The Mercury Laser will be the first integrated demonstration of laser diodes. crystals. and gas cooling within a scalable laser architecture.

This report is intended to summarize the progress accomplished during the first three years of the project. Due to the technological challenges associated with production of $900 \mathrm{~nm}$ diode-bars. heatsinks. and high optical-quality Yb:S-FAP crystals. the initial focus of the project was primarily centered on the $R \& D$ in these three areas. During the third year of the project. the $R \& D$ continued in parallel with the development of computer codes. partial activation of the laser. component testing. and code validation where appropriate.

\section{Introduction and Motivation for Building the Mercury Laser}

When neodymium laser ions were first doped into a crown silica glass in the early sixties it was soon realized that $\mathrm{Nd}$ :glass could offer many attributes ideally suited to the requirements of an inertial confinement fusion (ICF) laser. The advantages of flexibility in pulse format, wavelength, and spectral width with flashlamp-pumped $\mathrm{Nd}$ :glass lasers have pushed the progress towards unraveling the physics of ICF and to enabling the field of high energy density science. Since the "Janus" flashlamp-pumped $\mathrm{Nd}$ glass laser (Fig. 1) was built in the mid-1970's at LLNL, there was a belief that a scaleable architecture had been identified. for which millisecond-regime flashlamp-pumping could be employed to generate the needed nanosecond-scale pulse to irradiate the target. Since then over five major laser facilities have been developed, each progressively more energetic and culminating with the construction of the multi-megajoule National Ignition Facility. With flashlamp-based systems, the slow shot rate of once every few hours limits the number and type of experiments and applications that can be pursued. This limitation need no longer be imposed by the laser technology as first conceptually assembled in the early 1980s by Krupke and Emmett. ${ }^{1,2}$ The Mercury laser described in this report represents the development of a new class of high repetition-rate fusion lasers and will produce the first rep-rated solidstate fusion laser facility.

Gas-cooled diode-pumped Yb:crystal lasers are envisioned to be the next-generation ICF solid state laser system. NIF and the advanced diode-pumped solid state lasers (DPSSLs) discussed herein nonetheless share a great deal of common features, especially with regard to fundamental issues: multipass amplifiers, laser propagation, energy storage, extraction, pumping, linear and nonlinear wavefront distortions. frequency-conversion, and beam-smoothing. The DPSSL approach builds on the last two decades of solid-state laser development but also adds several imposing challenges -- repetition rate, reliability, efficiency and cost. Flashlamp-pumped solid-state lasers built for ICF studies have been optimized for the $\$ /$ Joule figure-of-merit and for their ability to match the target requirements. They are inherently single-shot devices, requiring several hours to recover from thermal distortions. The goal of developing ICF lasers with high repetition rate and efficiency has not yet been significantiy addressed. Solutions to these issues are:

- $\quad$ Employing near-sonic helium cooling of the laser slabs

- Trading the flashlamps for low-cost, large-size laser diode arrays

- Using laser Yb:crystals with greater energy storage and thermal conductivity than $\mathrm{Nd}$ :glass 
Laser diode arrays represent an enabling technology for realizing solid-state lasers for ICF. Not only are the diode technical pertormance specifications such as brightness more demanding than what is currently available. the diode array manufacturing costs will eventually have to be reduced by an order of magnitude. Developing a suitable diode package has been a major focus of our effort.

The goals of the crystal growth efforts for the Mercury project are to produce high quality $\mathrm{Yb}: \mathrm{S}-\mathrm{FAP}\left[\mathrm{Yb}^{3+}: \mathrm{Sr}_{3}\left(\mathrm{PO}_{4}\right)_{3} \mathrm{~F}\right]$ crystals to serve as the gain media. The Mercury Laser gas cooled architecture is being designed in a modular format. in which the laser slabs are mounted in vane elements supporting the gas-tlow. Importantly. this cooling technique is scaleable to larger aperture-size and hence greater energy/pulse. Gas-cooled laser architectures offer the promise of good beam quality due to minimal thermal distortion simultaneous with other essential parameters such as high peak power. reprated operation. high wall-plug efficiencies. and beam smoothness. The Mercury Laser is the first step in integrating these new approaches. and in producing new capabilities for irradiating ICF targets. These new facilities with rep-rated capability will provide a paradigm-shift for experiments and measurements in high energy density physics.

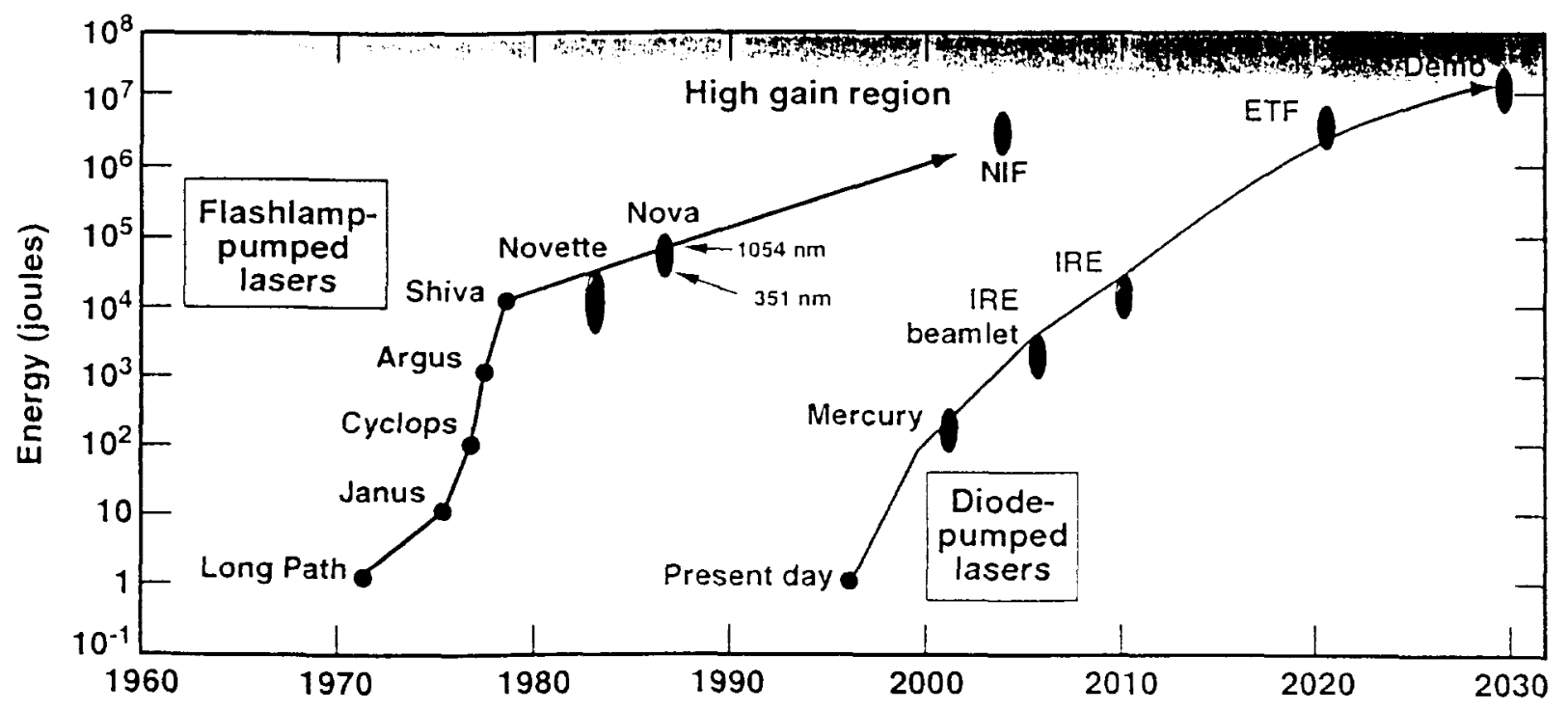

Fig. 1 Plot of the energy from flashlamp-pumped $\mathrm{Nd}$ :glass lasers as a function of time. and the potential time-line for diode-pumped laser development. 


\section{Conceptual Layout of the Mercury Laser}

The conceptual design for the laser system is shown in Fig. 2. The overall layout employs an angularly multiplexed scheme with a front-end laser and two gas-cooled amplifiers. The front-end laser system will provide temporally and spatially shaped pulses with energies up to $100 \mathrm{~mJ}$. In addition. the frequency spectrum will be conditioned to have $>30 \mathrm{GHz}$ of bandwidth to help mitigate intensity modulation generated from optical Fresnel reflections. The low energy input beam will be injected offaxis and near the focus to take advantage of angularly multiplexed concepts which avoid the use of optical switches (i.e. Pockel cells or Faraday rotators) at large apertures and high average powers (Fig. 3 ). A deformable mirtor either placed at the end of the amplifier path (as shown) or within the reverser optics path. will to correct tor low order (i.e. thermal) wavefront distortions incurred during amplification. After the beam has reflected back from the deformable mirror it returns to a different spatial location near the focus. A series of mirrors will be used to reinject the beam back into the amplifier chain for two final passes. The relay planes are located at the amplifier heads. detormable mirror. and within the reverser path. The amplitication stages are accomplished through four passes of the beam through two gas-cooled amplitier head assemblies.

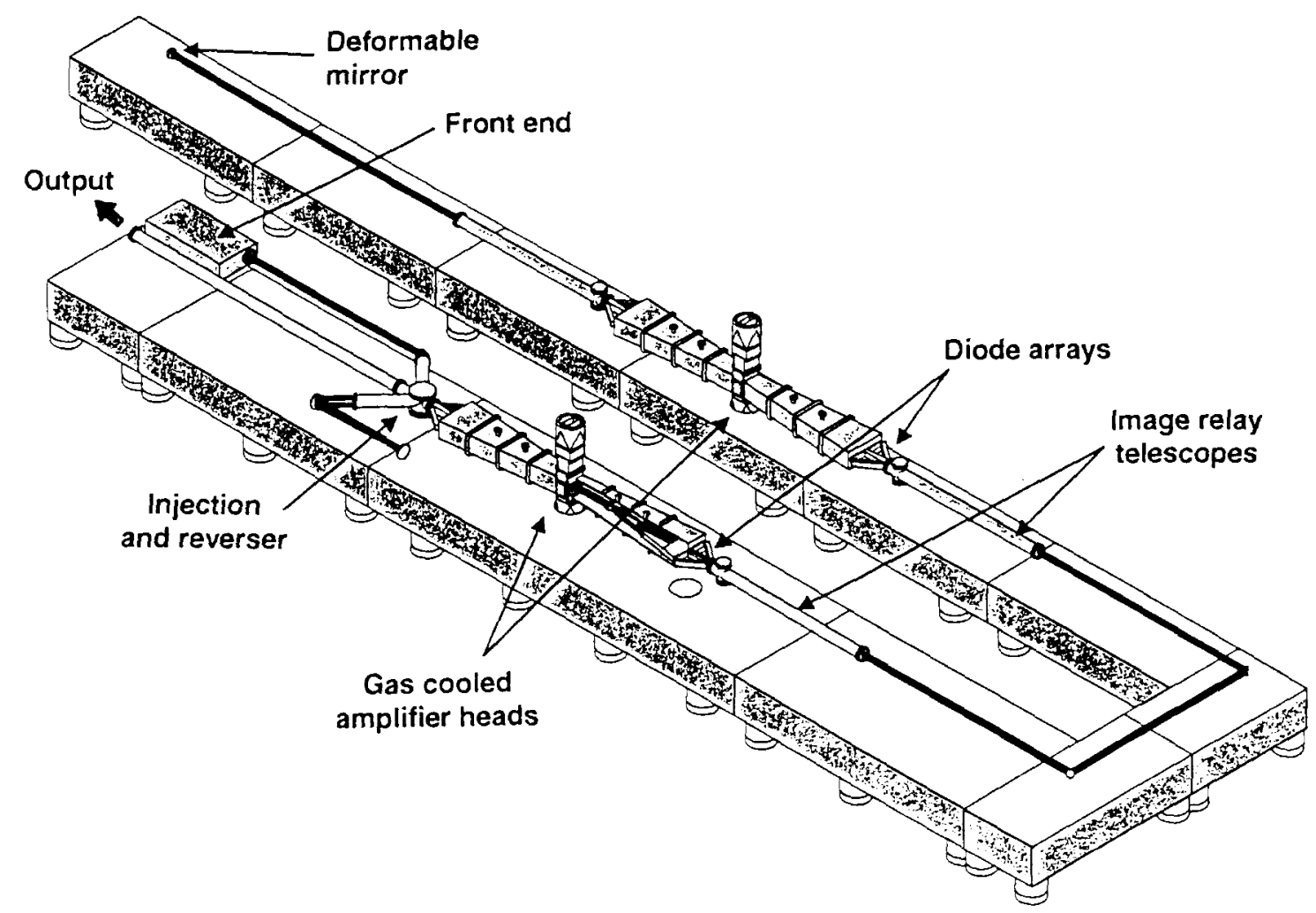

Fig. 2 Mercury laser system layout.

The pump delivery design has undergone a modification from the original baseline design in order to improve the diode pumping efficiency, scalability and robustness of the system. Since the design change took place after a small number of baseline optical components were purchased. we took advantage of the available hardware in order to benchmark critical codes (pump and gain) and increase confidence in our models and assumptions. 


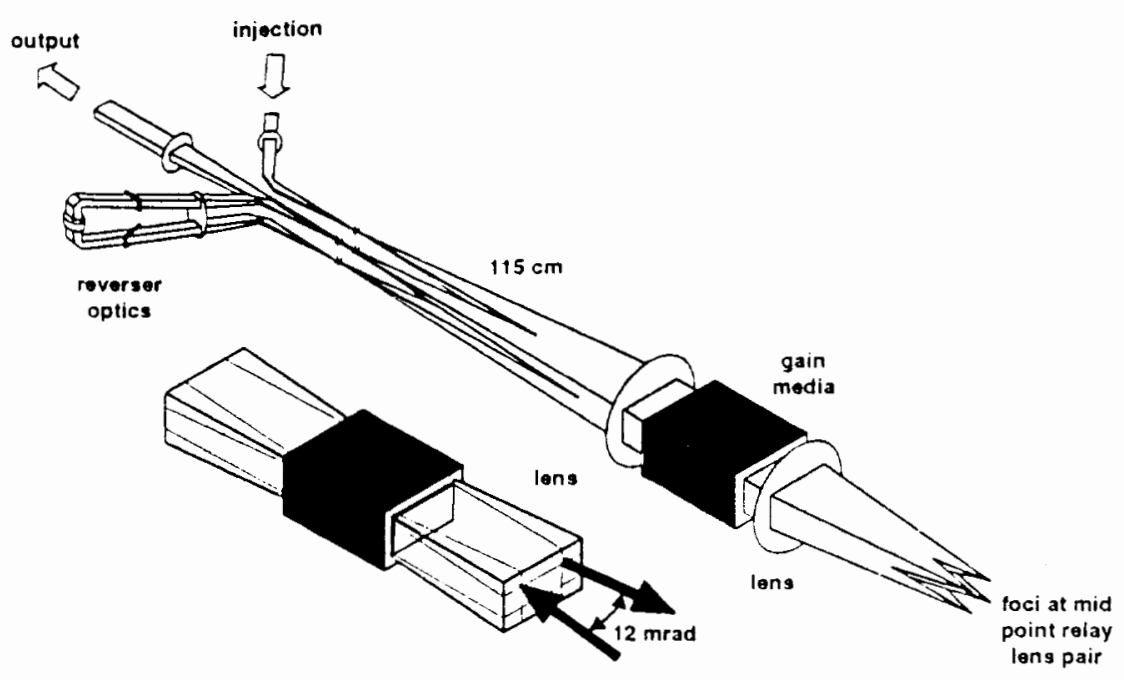

Fig. 3 The small multiplexing angles allow for a high fill factor in the amplifiers.

A detailed picture of the original baseline pumping geometry is shown in Fig. 4. The amplifier head will be optically pumped from both sides. The dual pumping design allows for more uniform pumping and thermal loading on the crystals. The light from the diode array light is first condensed with a hollow lens duct ${ }^{3}$ followed by a hollow non-concentrating element that homogenizes spatial profile of the pump beam. Both the duct and the homogenizer are coated on the inside surfaces with a protected silver coating. The silver coating was chosen for its high reflectivity ( $>99 \%)$ at grazing incidence angles. The duct and homogenizer also serve to contain stray diode light. The light emerging from the output of the homogenizer is imaged onto the gain media with a set of four SF-6 glass condenser lenses designed to minimize the spatial aberrations. The angled dichroic beam splitters allow the pump beam to pass through the optic and into the amplifier head while allowing the extraction beam to be reflected. The size of the dichroic was reduced to lower fabrication costs and more importantly increase the transfer efficiency by only placing the coating in a region where the laser light was expected to reflect off of the splitter.

The over all pump efficiency of the baseline system was measured to be $\sim 80 \%$ with the condenser lenses contributing to the majority of the transport losses $(86.8 \%)$. In assessing the baseline design more carefully, we found that the high $\mathrm{f}$-number condenser lenses created significant pincushion distortion in the relayed image of the diode light. Furthermore, in assessing the probability of damage with propagation code runs, we found that optics place away from relay planes, such as the dichroic splitter, were more at risk. In particular, the intensity modulation on the dichroic splitter was sufficiently high enough to cause damage. Consequently, if the dichroic beamsplitter were to fail, the extraction beam would propagate directly into the diode array and cause catastrophic damage. Finally, in assessing the baseline optical layout in the context of a bundled beamline for IFE. we found a number of problems with the ability to gracefully combine several apertures into a single compact beamline. 


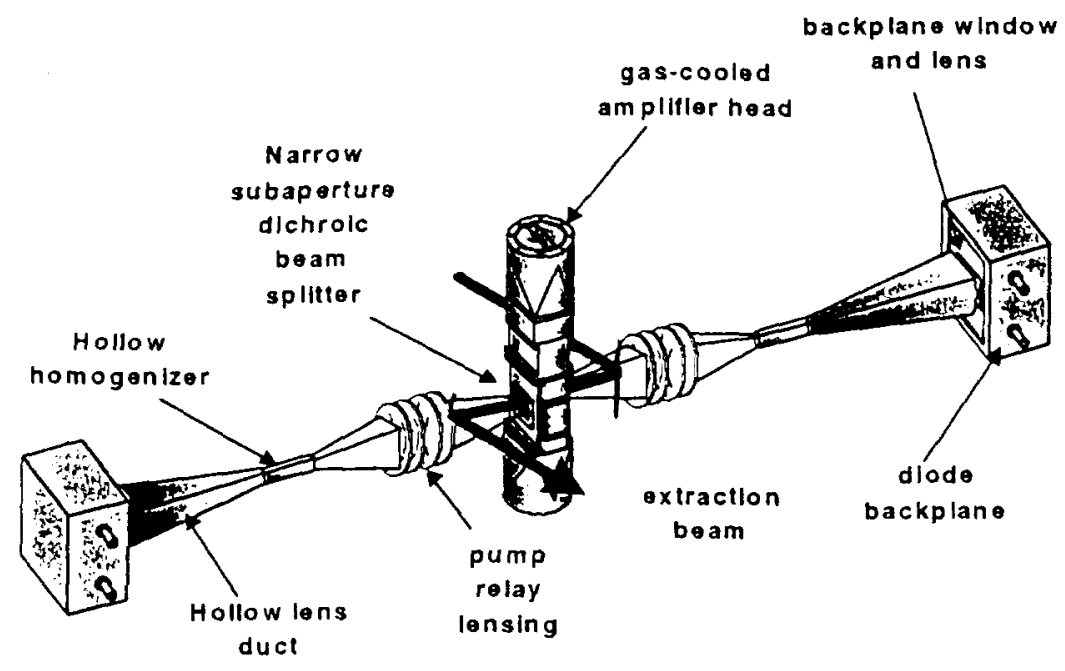

Fig. 4 Baseline pumping arrangement.

The advanced design eliminates the condenser optics and the dichroic splitter, thus increasing the overall pump transport efficiency while reducing the number of components. The main difference between the baseline and advanced designs is that the backplane hardware must be divided into two arrays to allow the laser beam to pass through the middle of the diode array (Fig. 5). The split in the diode array decreases the pump brightness and uniformity. We can compensate for the lower brightness by the overall higher transport efficiency. In addition, the homogenizer can be lengthened to help smooth the beam profile. Finally, in order to keep the separation between the diode arrays as small as possible, the relay telescope lenses were placed next to the amplifier thereby allowing the extraction beam to focus just beyond the diode arrays. The placement of the telescope lenses next to a relay plane helps prevent damage from occurring in the final amplification stages. Finally, this design scales more gracefully to larger apertures and beam bundles.

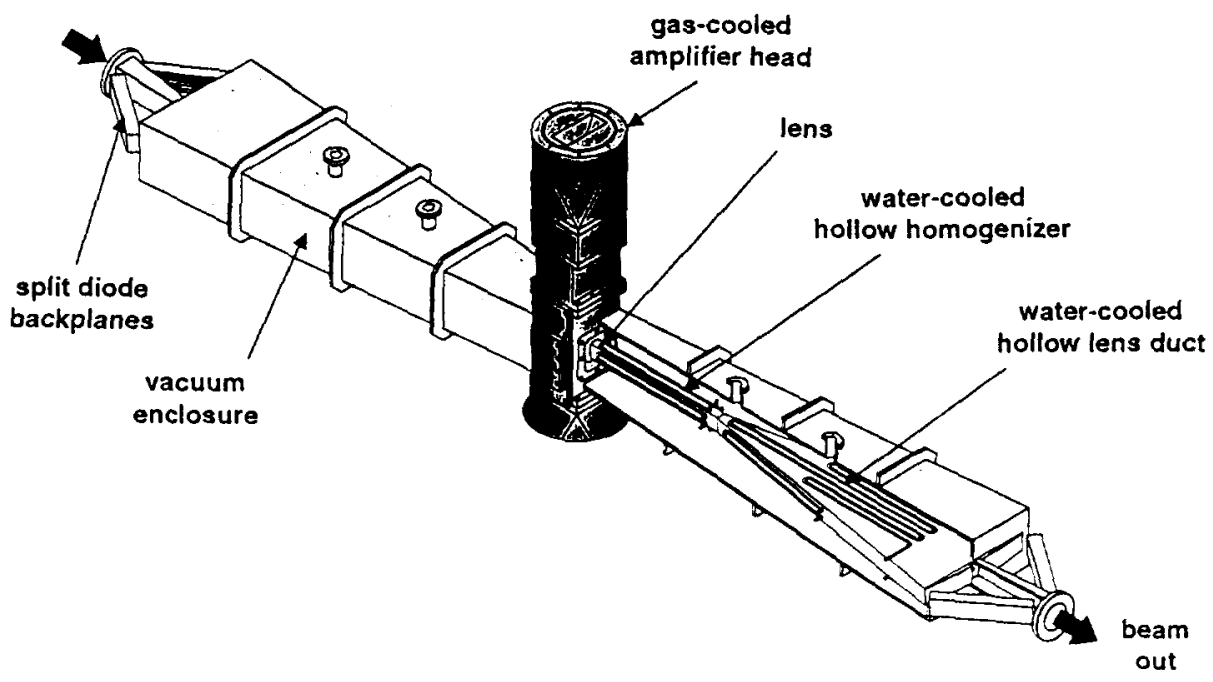

Fig. 5 Dual pumping geometry with the baseline and advanced pump delivery designs. 


\section{Laser Requirements}

The primary performance goals for the Mercury Laser are:

- $10 \mathrm{~Hz}$ repetition rate

- $10 \%$ efficiency

- $\quad 1.0+7 \mu \mathrm{m}$ wavelength $(1 \omega)$

- 2-10 nsec pulsewidths

- 100 Joules energy

- $\quad 5 x$ diffraction-limited beam quality

- $\quad>10^{7}$ shor lifetime

- Scalable architecture

These requirements are based on an in-depth study of IFE beamline requirements ${ }^{31}$. In particular the efficiency, lifetime, and scalability requirements have been particularly challenging. When completed Mercury will be the highest energy/pulse diode-pumped laser ever built by an order of magnitude. It is noteworthy that the $100 \mathrm{~J}$ energy is the same as that of the Janus Laser. which is based on flashlamppumped Nd:glass and originally built in 1973. In addition. the Mercury Laser will be upgradable to $3 \omega$ generation, and to picosecond operation. We have begun assessing future requirements (such as pointing stability, pulse shaping, etc.) that would make Mercury an attractive shots on demand facility for high energy physics experiments and offline NIF experiments to assess damage. debris studies and diagnostics calibration. 


\section{Major Architectural Elements}

\section{A. Gas Cooled Amplifier}

The Mercury gas cooled amplifier design was predicated on three important design constraints:

- The design must minimize cooling induced optical distortions in the laser slabs

- The cooling flow must have minimal impact on laser performance

- The design must be scaleable and consistent with IFE system requirements

Miny general aspects of the design are based on earlier work where the concept of face cooling slabs was first identified. applied, and the fundamental principles developed. The earliest study involved the gas cooled disk amplifier testbed (GCSTB). ${ }^{4.5}$ A fundamental result of this work was the identification of helium as the only viable candidate for the cooling gas because of its significantly reduced optical distortions (when compared to other gases. such as nitrogen) and because of the improved heat transfer rate. In this earlier work, acceptable flow regimes for IFE applications were identified. primarily from the standpoint of the impact of the cooling system power consumption on the overall efficiency of an IFE plant. $^{+}$

More recently, these cooling concepts were applied a single diode-pumped laser slab, referred to as the gas cooled diode-pumped laser (GCSL). ${ }^{6}$ This was the first diode pumped laser to employ gas cooling. This demonstrated the utility of face cooling with helium at heat fluxes typical of the Mercury laser and in a channel architecture that was related to that which is used in the Mercury design.

A comparison of the Mercury laser cooling requirements with the bounds established in previous work is given in Table 1. Efforts have been made to ensure that the cooling channel thickness, flow Mach number, and gas pressure are at levels consistent with those employed in previous studies as well as consistent with projections for an IFE plant design. ${ }^{7}$ The flow Mach number is limited to an upper bound of approximately 0.1 in order to minimize the impact of cooling flow power consumption on system efficiency.

Table 1 Comparison of cooling requirements.

\begin{tabular}{|c|c|c|c|}
\hline Parameter & Mercury laser & $\begin{array}{c}\text { Gas cooled disk } \\
\text { Amplifier test bed } \\
(1992)\end{array}$ & $\begin{array}{c}\text { GCS diode pumped } \\
\text { KJ-class beamline }\end{array}$ \\
\hline Cooling gas & helium & nitrogen & helium \\
\hline Gas pressure & $4 \mathrm{~atm}$ & $2-4 \mathrm{~atm}$ & $4 \mathrm{~atm}$ \\
\hline Flow Mach no. & $\sim 0.1$ & $0.05-0.3$ & $\sim 0.1$ \\
\hline Channel thickness & $\sim 1 \mathrm{~mm}$ & $1-5 \mathrm{~mm}$ & $\mathrm{~mm}$ \\
\hline Surface heat tlux & $0.5-1.5 \mathrm{~W} / \mathrm{cm}^{2}$ & $1-5 \mathrm{~W} / \mathrm{cm} 2$ & $0.3-3 \mathrm{~W} / \mathrm{cm}^{2}$ \\
\hline$\%$ of fracture & $\sim 10 \% \mathrm{max}$ & - & up to $30 \%$ \\
\hline
\end{tabular}


One fundamental requirement of the architecture is that the slabs in the amplifier head be nearly parallel to one-another. as depicted in Fig. 6a. A cooling channel exists between slab pairs through which helium flows to remove the waste heat deposited in the slabs. On either end of the "slab stack" is a window through which both the diode light and laser light pass. To provide symmetric cooling for the slabs adjacent to the windows, a cooling channel exists between the window and adjacent slab. A fundamental aspect of this approach is that cooling considerations do not dictate the number of slabs in the stack. That parameter is entirely dictated by laser performance issues. This "slab stack" is housed in the amplifier head. as shown in Fig. 6b. with each slab potted into an aerodynamic vane element as shown in Fig. $6 \mathrm{c}$.

(a)

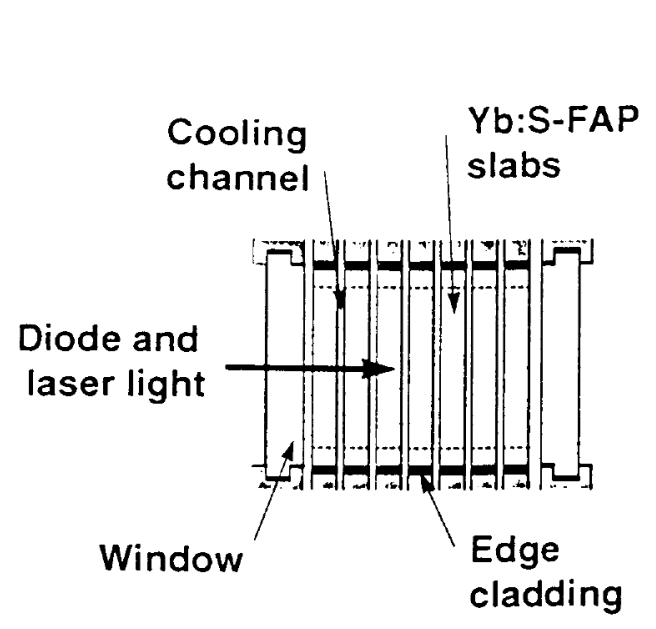

He gas

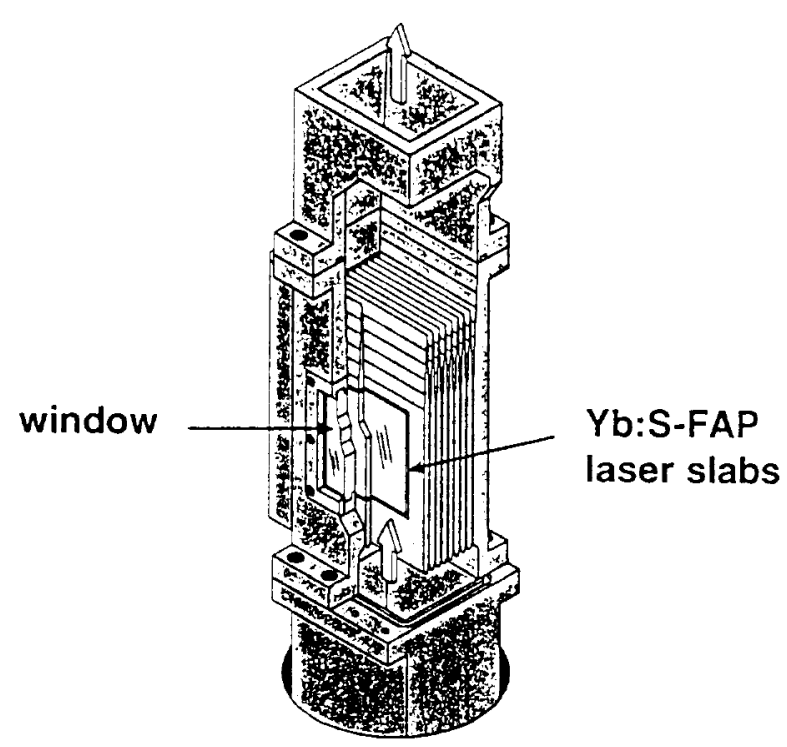

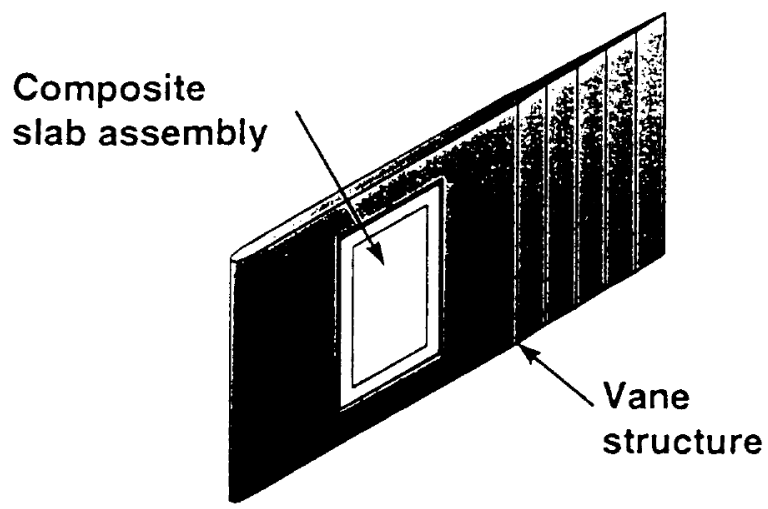

(c)

Fig. 6 An overall picture of the amplifier architecture. (a) A representation of the cross-section through the assembly showing the cooling flow channels. (b) A cut-away of the amplifier head showing the slabs and vane assembly. (c) An individual vane showing the potted slab. 
There are a number of important details of the laser head design that are dictated by flow considerations and the modularity requirement. Fig. 7 gives a schematic cross-section of the Mercury amplifier head. Upstream of the vane elements is a nozzle section where relatively slow velocity cooling gas is accelerated. Each pair of vanes forms an aerodynamic channel consisting of an entrance section where the gas is further accelerated. the constant area channel section that contains the slab. and the trailing diftuser section where the flow is decelerated. The diffuser is broken into two regimes: (1) the diffuser elements that are integral to the vane assembly. and (2) diffuser elements downstream of the vane assembly.

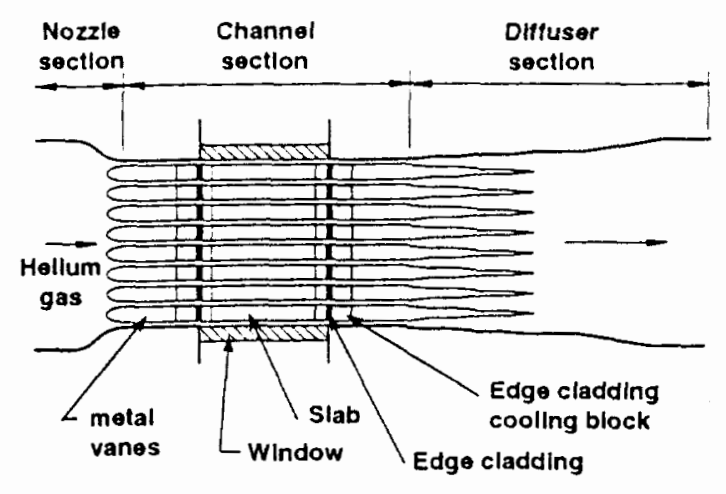

Fig. 7 A pictorial representation of the flow features of the Mercury amplifier head design. Shown are the nozzle section where the tlow is accelerated to near Mach 0.1 , the constant area channel section that includes the laser slabs, and the diffuser section where the flow is decelerated.
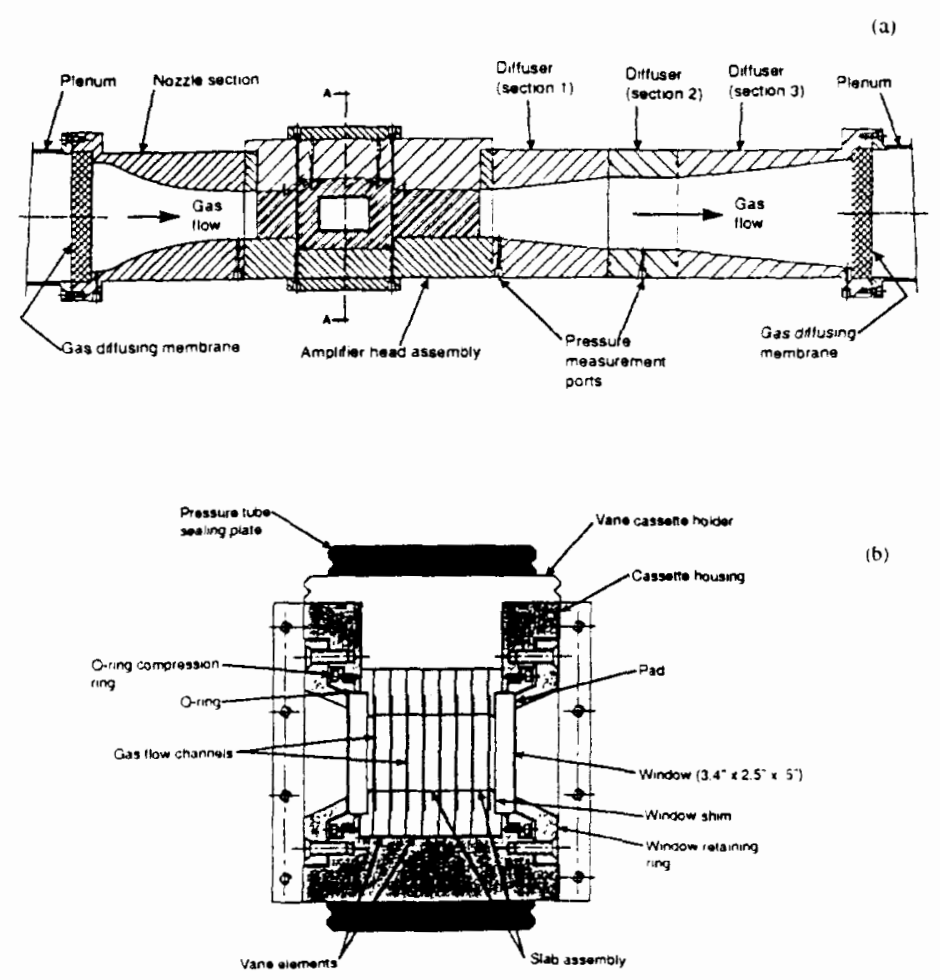

(b)

Fig. 8 Further details of the Mercury amplifier head. (a) A cross-section through one of the flow channels, showing details of the nozzle section and the diffuse sections downstream of the vanes. (b) A side view of the amplifier hardware that holds the window and vanes. 
The actual character of the flow elements used in the Mercury amplifier head are given in the cross-sections of Fig. 8. Referring to Fig. 8a. besides the diffuser elements in the vane assembly. additional diffuser elements (diffuser sections 1.2. and 3) were place downstream do further decelerate the flow. The "gas diffusing membranes". which were merely porous elements, were placed at the entrance and exit to ensure spatially uniform flow entering the nozzle section and exiting the last diffuser section. Fig. 8a shows a cross-section through the vane region. This cross-section shows the window constraint and sealing mechanism that consists of an O-ring compression ring and a window retaining ring. The window is mounted so that it's inside surface forms a wall of the outside channel.

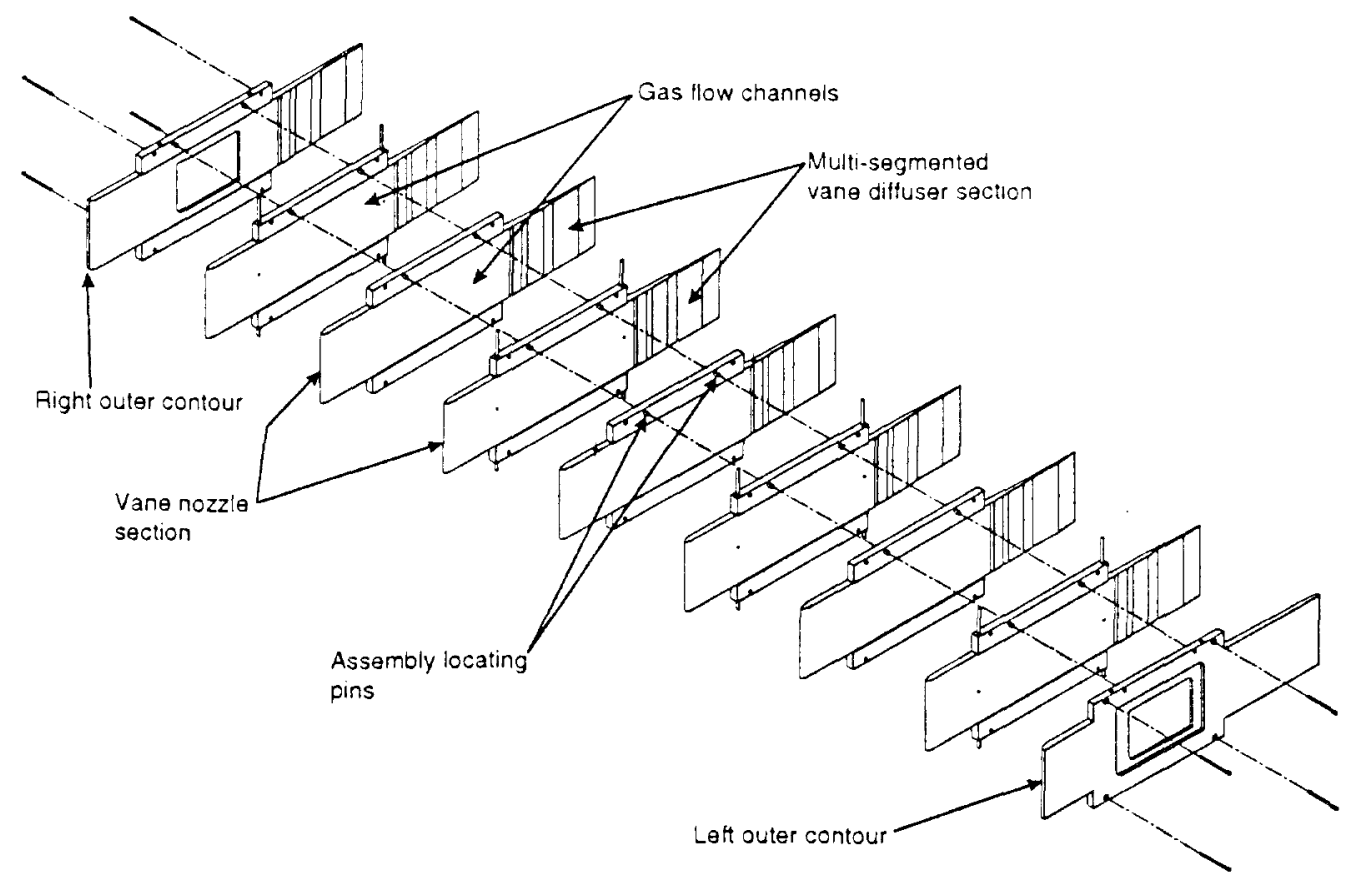

Fig. 9 An exploded view of the vane assembly, showing the 7 vane elements and the two outer contour elements that accept the window and provide the proper nozzle and diffuser profiles for the outer channels.

Assembly of the amplifier head is depicted in Figs. 4 through 11. Fig. 9 shows the manner in which the vane package is assembled using guide pins and retaining bolts. This assembly is then attached to the cassette holder plate as shown in Fig. 10a. with the entire assembly placed in the amplifier head housing (Fig. 10b). Seal plates are then attached to form the final pressure barrier (Fig. 10c). The windows are inserted into the amplifier head, as shown in Fig. 11, using the O-ring and window retaining rings.

\section{Design Analysis}

A key feature of this design is the diffuser sections. particularly those in the vane element region. In these regions the flow is decelerated for return to the heat exchanger and fan unit. This deceleration is necessary to ensure minimal pressure losses in the remainder of the system. Of concern during this deceleration process is the potential for flow instabilities to develop, such as separation that could induce 
(a)

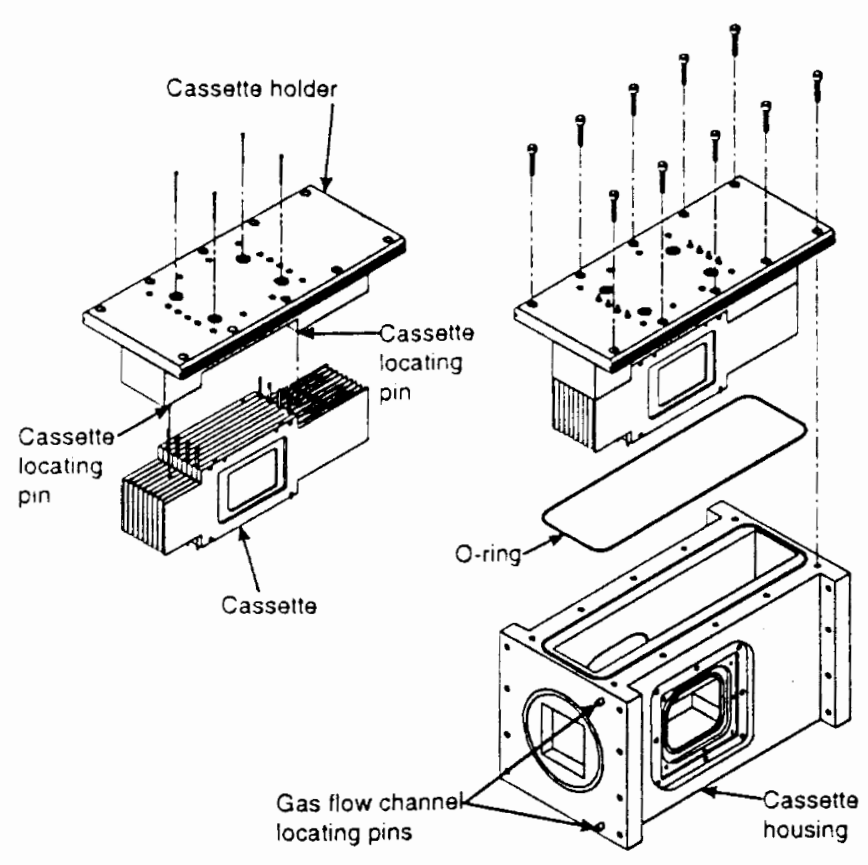

(c)

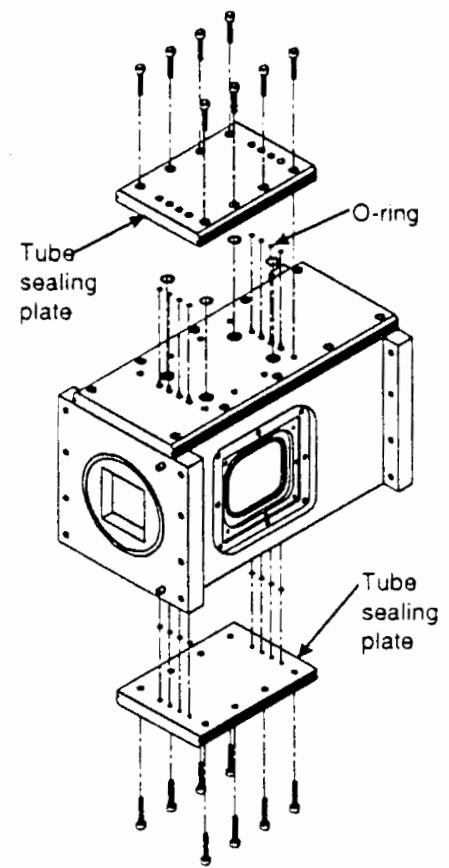

Fig. 10 Sequence of sketches showing the assembly of the amplifier head. (a) Joining of the vane cassette assembly to the cassette holder. (b) Insertion of the vane cassette into the amplifier head. (c) Attachment of the pressure seal plates.

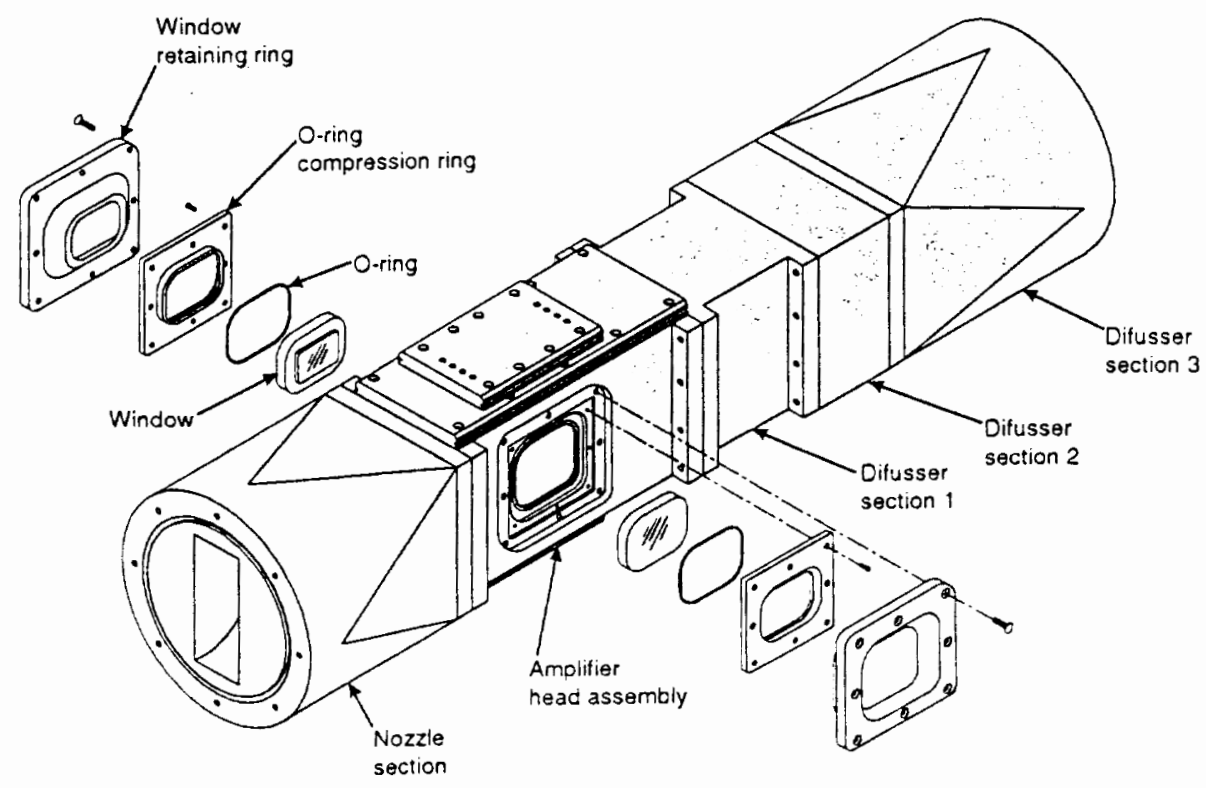

Fig. II Sketch showing final assembly of the windows into the Mercury amplifier head. 
mechanical vibration of the laser head. To properly design these diffuser sections. detailed finite element fluid dynamics calculations were pertormed that directed and quantified the design details. ${ }^{3}$ Fig. 12 gives details of the vane diffuser region that is formed between two vanes. The diffuser region consists of 5 expansion sections. separated by a constant width section that we term a relaxation region. A representative finite element calculation is shown in Figs. 13 and 14 . Fig. 13 shows velocity vector plots through two adjacent expansion sections. Careful examination of these plots shows that as the flow progresses through the expansion section. not only does the flow velocity reduce due to the increased flow area. but also there is a preferential tendency for reduction near the walls. This is quantified in Fig. 14. which gives velocity profiles across the channel at expansion section entrances and exits. The locations of the profiles are shown in Fig. $1+c$. Profiles " $a$ " and " $c$ " are taken at the expansion section entrances. while profiles " $b$ " and " $d$ " are taken at the expansion section exits. Fig. Itil gives the profile over the entire channel half-width. while Fig. $1+b$ isolates the region next to the wall. Starting at location "a". the velocity profile is characteristic of a fully developed turbulent profile in a two-dimensional channel. At the exit of the first expansion (curve "b"). the flow not only shows the effects of an overall deceleration, but at the wall there is a dramatic flattening of the velocity profile, which is indicative of a tendency toward separation. However. because there is no evidence of flow reversal. which would appear as negative velocities. we have not yet expanded to the point of separation. The purpose of the intermediate constant width section is to relax the flow, i.e.. allow momentum diffusion to fill in the velocity profile. This is seen in curve " $c$ " which once again shows characteristics of a fully developed profile. At the end of this "relaxation" section, the flow is once again ready to undergo the next expansion. which is terminated prior to the onset of separation. This process is repeat through 5 expansion sections in this diffuser design.

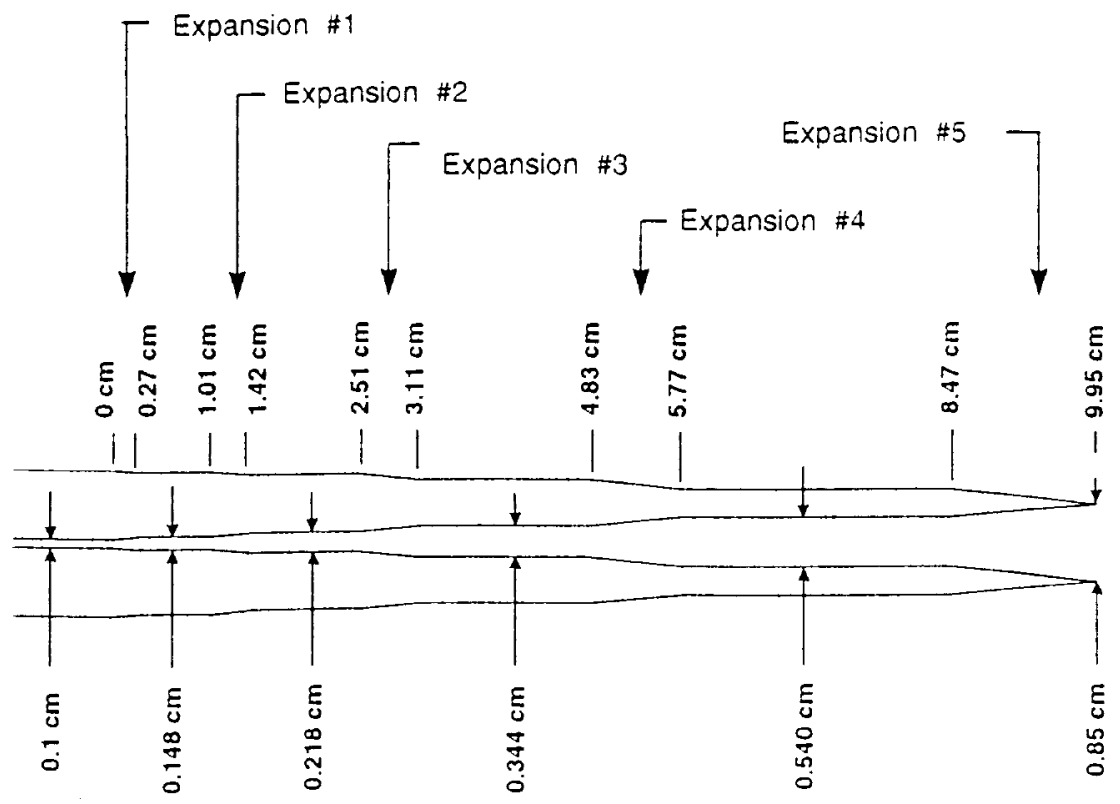

Fig. 12 Geometry and dimensional details of the vane diffuser segment. which consisted of five expansion regions separated by constant area relaxation zones. 

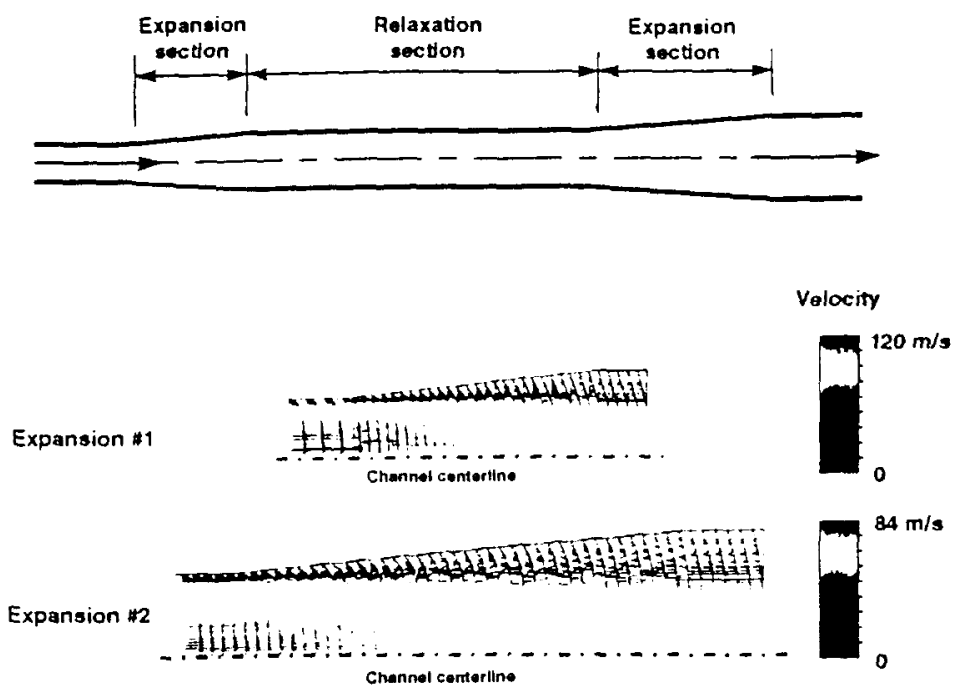

Fig. 13 Representative velocity vector plots in two expansion sections of the vane diffuser element. The plots show the deceleration of the flow as well as the profile development that results from the relaxation section.
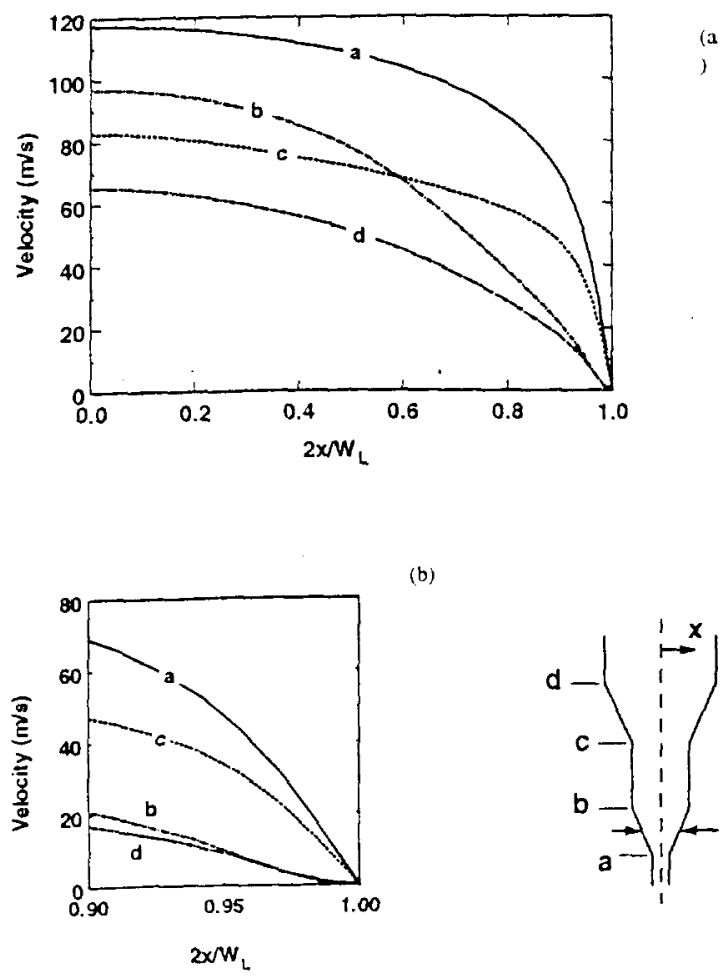

(b)

(c)

Fig. 14 Velocity profiles across the channel at the entrances and exits to two successive expansion sections in the vane diffuser element. (a) Profiles across the entire channel half-width. (b) Isolation of the velocity profiles in the near-wall region. (c) Locations of the profiles. 


\section{Flow Performance Experiments}

In this design, the 8 channel flows merge at the end of the vane diffuser region. Uniform pressure drops in the channels is required for this to be successful. Fig. 15 shows the measured pressure drop in the constant area section of each channel (Fig. 15a) and the channel-to-channel pressure differential at the channel exit (Fig. 15b). Referring to Fig. 15a. the average pressure drop is 0.775 psi. with a variation of $+0.056 \mathrm{psi} .-0.052 \mathrm{psi}(\mathrm{or}+7.2 \% .-6.7 \%$ ). This pressure variation translates to a velocity variation of $3.6 \%$ over the set of 8 channels. which is considered adequate to preclude formation of wake disturbances when the flows merge at the trailing edge of the vane.
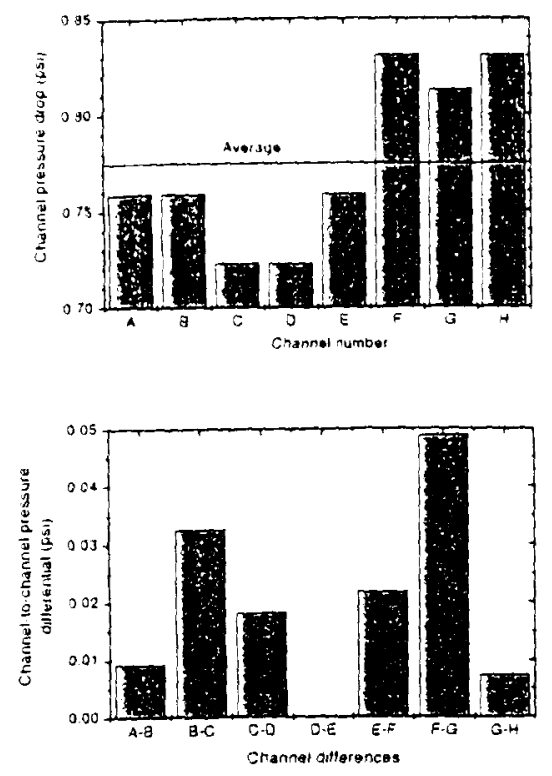

Fig. 15 Measured pressure variations in the channel region with solid vanes. (a) Individual channel pressure drops. (b) Exit pressure differentials.

Another important consideration is flow related vibration of the amplifier head and laser tables. Measurements found that the principle source of vibration was the cooling system mechanical equipment. such as compressors, fans, and pumps. ${ }^{9,11}$ Vibration measurements were made at 10 locations in the Mercury laboratory as shown in Fig. 16.

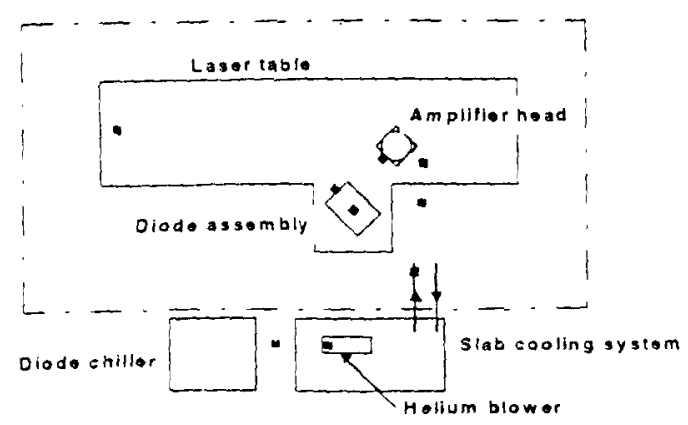

Fig. 16 Location of transducers used to measure the vibration frequency spectrum. 


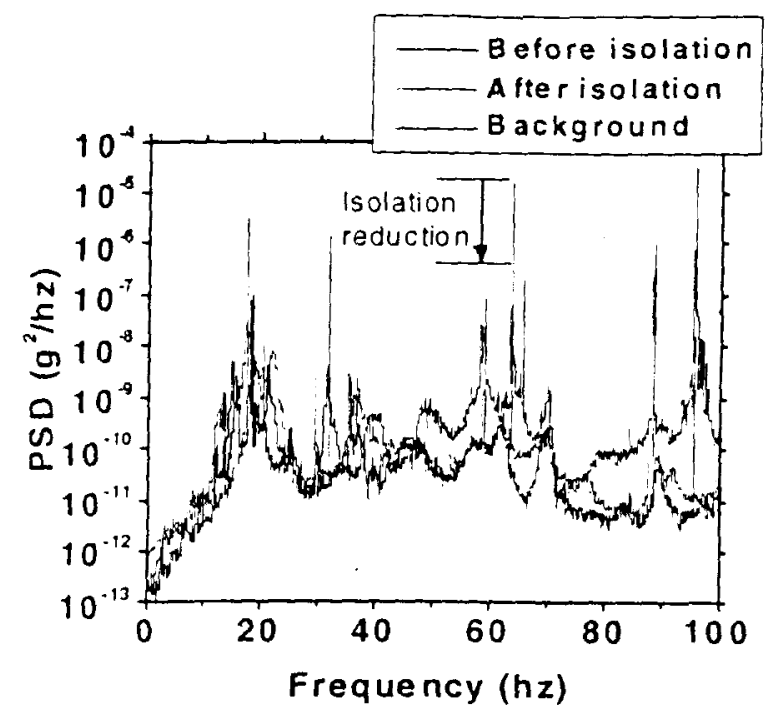

Fig. 17 Power spectral density (PSD) plots of the horizontal acceleration.

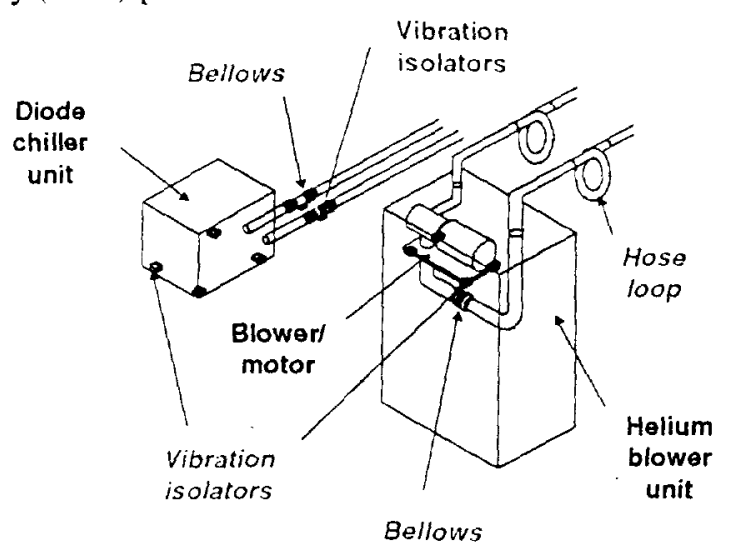

Fig. 18 Pictorial representation of the vibration isolation features incorporated into the Mercury gas supply hardware.

Fig. 17 gives power spectral density (PSD) plots of the horizontal acceleration on the laser table next to the amplifier head. The background curve is with the room HEPA filters on, and no other mechanical equipment associated with the Mercury laser activated. The "before" curves refer to measurements made with the helium recirculating system operational "betore" efforts were made to vibration isolate the helium drive system from the amplifier head and laser table. Evident in this curve are power peaks at $60 \mathrm{~Hz}$ and its harmonics. which is indicative of reciprocating machinery. The "after" curve refers to measurements made after isolation. The isolation features that were implemented are described in Fig. 18. They consisted of (1) placing isolation pads under the blower/motor assembly to reduce the transmission of blow vibration through the ground and to the table, and (2) inserting loops of flexible hose in both the supply and return lines to reduce the direct mechanical coupling of the blower to the amplifier head. Comparing the "before" and "after" curves shows at least a 2 decade reduction in the peak powers with vibration isolation. 
One tinal vibration point relates to the potential effects of potting slabs into the vanes. If irregularities exist that alter the channel profile. there is the potential for flow instabilities to develop. An uncalibrated accelerometer was placed on the amplifier head. with the acceleration spectrum measured with solid vanes and potted vanes as shown in Fig. 19.

\section{Solid Vane}

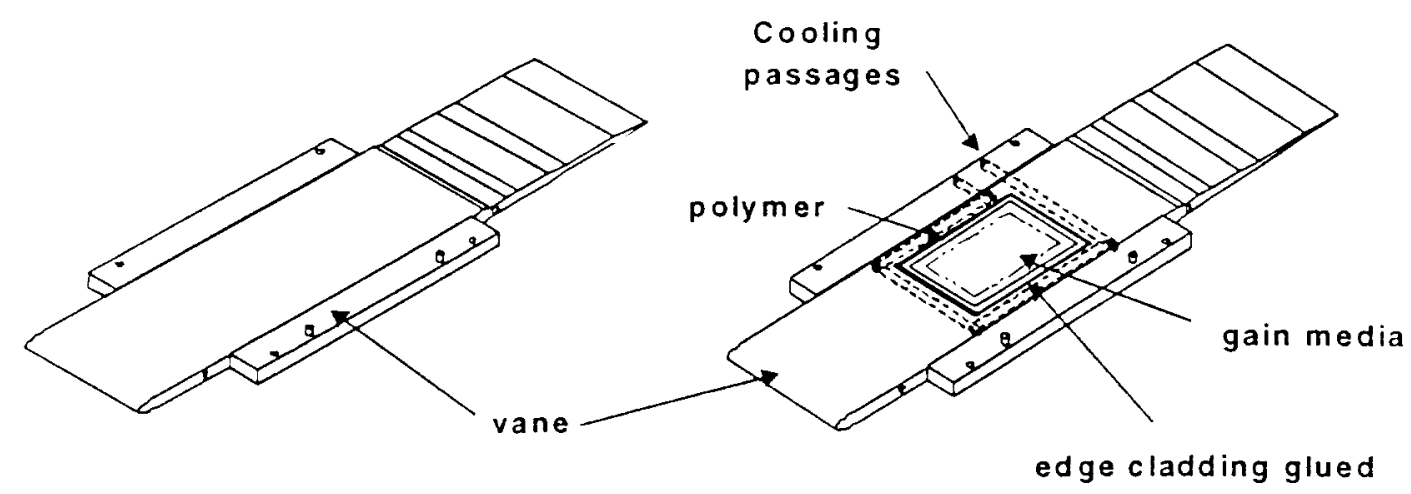

Fig. 19 The hollow vanes are potted with surrogate gain media in order to test the flow characteristics of the amplifier design.

A comparison of those measurements is given in Fig. 20. These measurements show negligible difference between the solid and potted flow conditions, which indicates that any instabilities from flow irregularities in the flow channel due to potting are not significant enough to be differentiated from the overall system vibration sources.

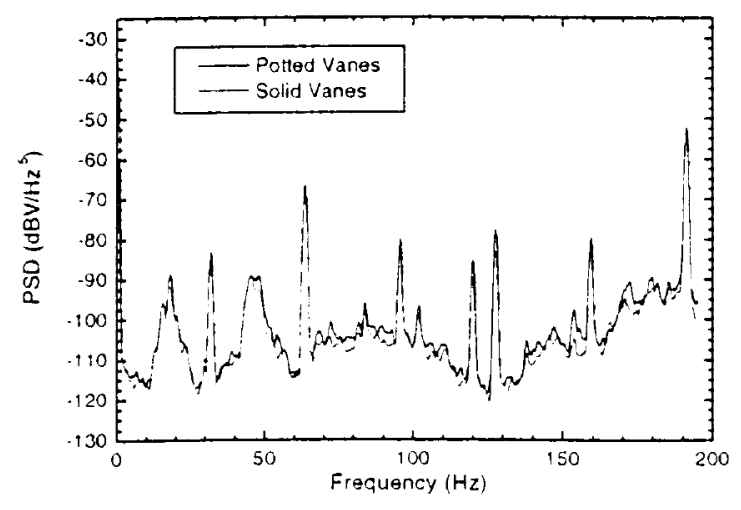

Fig. 20 Qualitative characterization of the impact of slab potting on system vibrations. These measurements were made with an uncalibrated accelerometer, so only a relative comparison is appropriate. 


\section{B. Pump Delivery System}

\section{Original baseline layout}

A schematic and picture of the baseline pump delivery hardware are shown in Fig. 21. A description of the individual components is discussed in detail below followed by a summary of the experimental data.
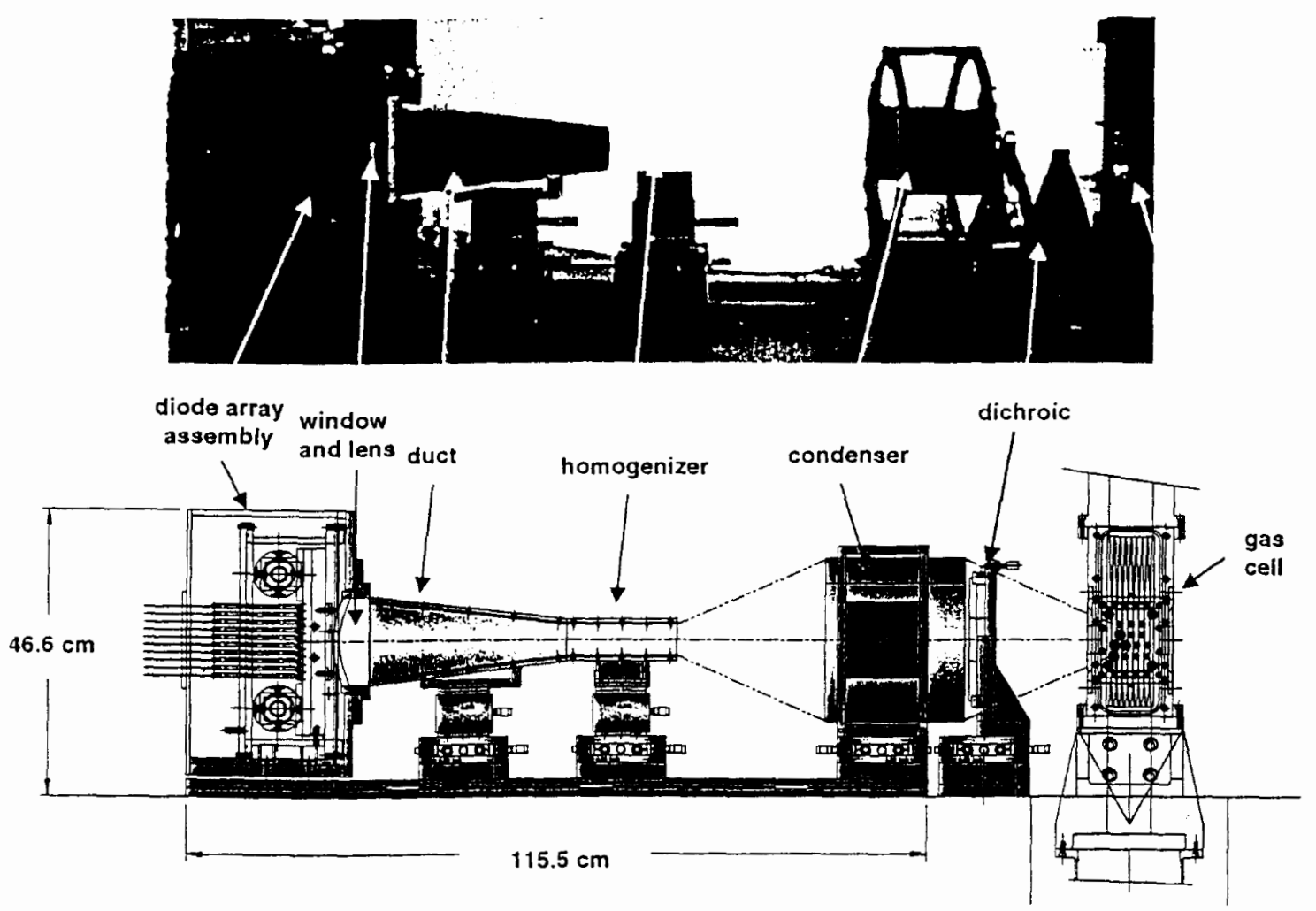

Fig. 21 Baseline pumping scheme. The yellow shaded triangle illustrates the diode light collection angle of the condenser lenses.

\section{Diode array}

The diode array and its double enclosure appear on the left. The array is capable of holding up to 40 tiles ( 1600 diodes) to produce a combined peak-power output of $160 \mathrm{~kW}$ at $900 \mathrm{~nm}$. The tiles are mounted to a copper backplane. which contains the cooling channels and electrical feed-throughs. The coolant. hydrotluoroether (HFE7100). allows the diodes to reach temperatures as low as $-40^{\circ} \mathrm{C}$. The option of operating the diodes at low temperatures allows for wing-pumping the surrogate $\mathrm{Nd}$ :glass slabs. The diodes are typically operated at $12^{\circ} \mathrm{C}$ for $\mathrm{Yb}: \mathrm{S}$-FAP. In order to avoid condensation from occurring on the diodes. the backplane is sealed with two optics to provide the double enclosure and purged with nitrogen. An AR-coated thin window and a rectangular lens provide the primary and secondary enclosures respectively. 


\section{Hollow duct and homogenizer}

The lens duct and homogenizer are the primary components that modify the spatial and angular distribution of the diode light. The optimal design of these components is based on simultaneously meeting the following criteria:

- High transport efficiency

- Uniform angular distribution with minimal increase in divergence

- Uniform pump beam protile

The lens duct is a non-imaging optic and serves to concentrate the diode light to a $3 \times 5 \mathrm{~cm}^{2}$ aperture. The lens duct consists of a rectangular lens and a hollow metal light guide or duct. The lens duct combination serves to concentrate the light to the required aperture with a uniform angular distribution. The second optic. a homogenizer. is used to smooth the spatial near field profile without altering the angular distribution. The homogenizer consists of four parallel plates. The internal surfaces of the optical guides can reflect the $900 \mathrm{~nm}$ light with greater than $98 \%$ efficiency. Both the duct and the homogenizer are fabricated from polished aluminum plates with a protective silver coating.

\section{Condenser}

The condenser lenses are made of a high index SF- 6 glass. The condenser lenses were designed to image the outpur of the homogenizer onto the first slab with minimum distortion and losses. Since the Fnumber was extremely low due to the high collection angle. the optics were challenging to design for high collection efficiency and high beam quality. We chose a t-lens scheme to help reduce optical aberrations; however, we still observed pincushion effects as well as low transport efficiency $(86 \%)$

\section{Dichroic splitter}

The dichroic beam splitter is uses a complex multilayer dielectric coating to reflect the $1047 \mathrm{~nm}$ extraction beam and transmit the $900 \mathrm{~nm}$ pump light. The dichroic splitter allows the main extraction beam to exit the pump path and be directed into the relay telescopes. The manufacturability of the splitter was very difficuli due to the small separation in wavelength between the pump and laser beam and the low losses desired at both wavelengths. Although the manufacturer was able to produce an acceptable optic in terms of losses, experimental results showed several waves of distortion that were probably due to dielectric layer stress-induced surtace deformations.

\section{Ray trace modeling and experimental data}

The ray trace modeling is accomplished by using a commercially available sottware package called Opticad on a desktop computer. The Opticad software allows one to build solid models of optical components and then nonsequentially trace rays through the optical system. The data dump files consist of intensity distribution profiles at user specified locations. In order to accurately mock up the V-BASIS diode light distribution for Mercury. the experimentally measured fast and slow axis far field data is imported directly into the code. The code does not take into account interference or diffraction phenomena. Nevertheless. the $>100.000$ emitters from each diode array can be considered temporally incoherent with respect to each other and interference effects can be neglected. The user specifies the number of rays to launch for each computer run. Typical launch numbers involve over 2 million rays and several hours to run. The code was benchmarked with experimental data taken in the laboratory with the baseline pump delivery optics as shown in Fig. 22. As can be seen from the data. the largest loss in efficiency is from the set of condenser lenses. Near field images were taken at several locations. With $1 / 4$ of the pump light turned on. several images were captured in different planes. As can bee seen from Fig. 23 the model and experimental data are in close agreement. 


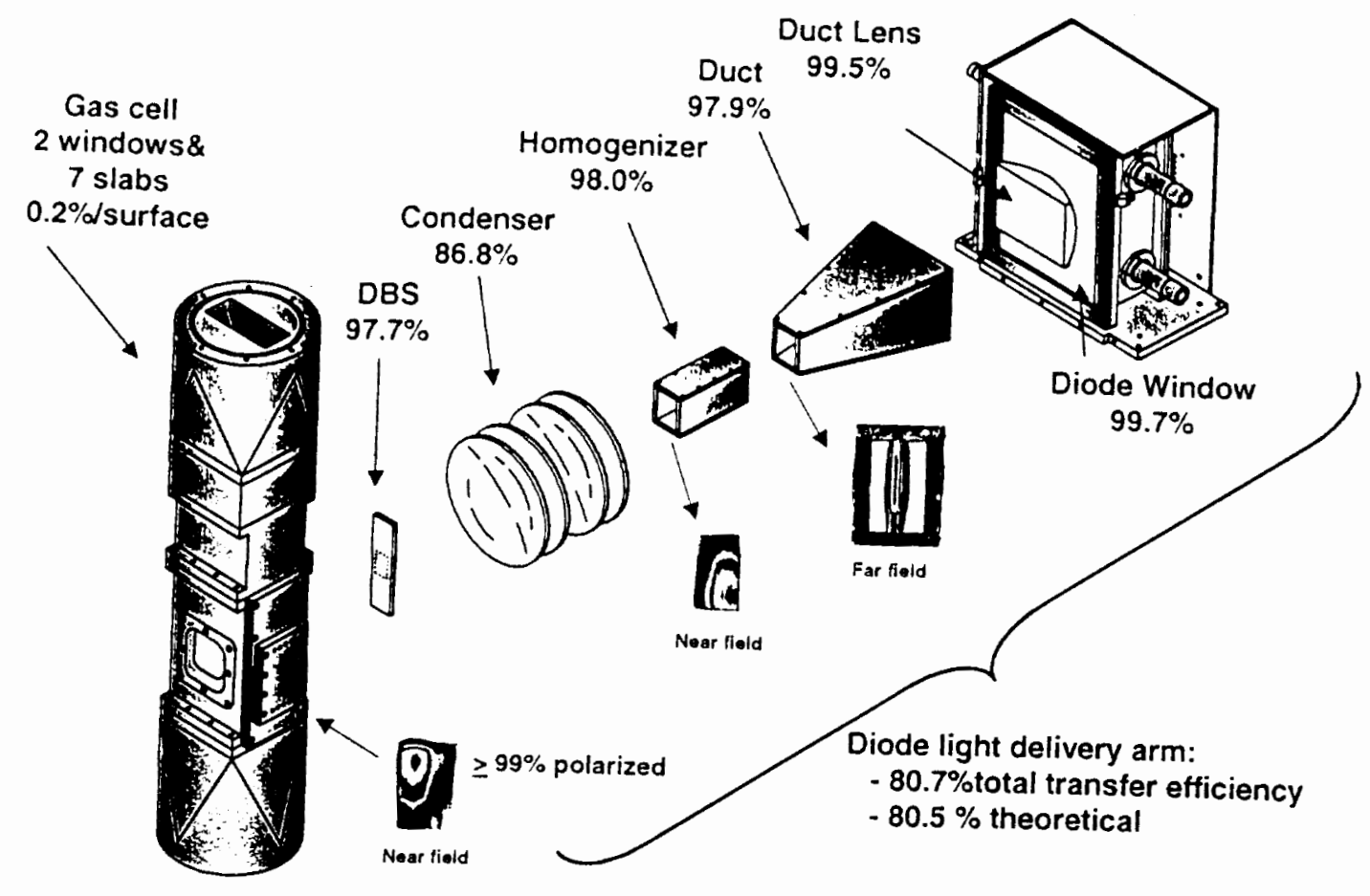
Fig. 22 A summary of the measure transport efficiencies of each optic in addition to the output diode light
profiles at the exit of each optic
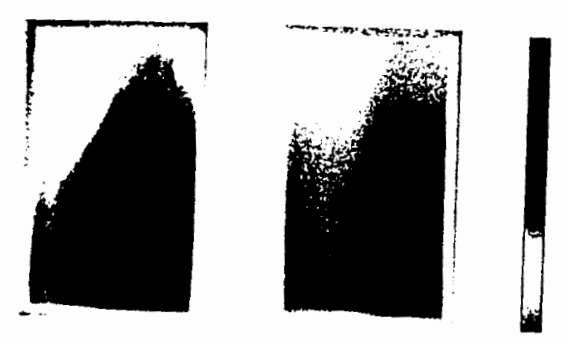

Experimental Data Model

Fig. 23 The model and experimental data agree reasonable well. Only one quadrant of the pump array was
utilized. 


\section{Advanced Pump Design}

An advanced pump design was considered for several reasons:

- The transition from BASIS to V-BASIS heat sink architecture (Section 6)

- Reduction of damage risk to optical components

- Preference for a more robust and scalable architecture

The transition from BASIS to V-BASIS heat sink architecture created a new challenge in designing the pump delivery hardware since the diode light emits at $-55^{\circ}$ from the normal axis compared to a $90^{\circ}$ direction with BASIS. This required us to cant the backplane at a $55^{\circ}$ angle to keep the light parallel to the optical axis. Although the overall brightness $\left(\mathrm{W} / \mathrm{cm}^{2} / \mathrm{sr}\right)$ remains the same, a redesign of the pump delivery hardware was required to accommodate the new backplane geometry. The concept of splitting the backplane into two components. removing the imaging optics. and allowing the beam to be completely collinear with the pump light was successfully demonstrated in a smaller IJ laser system."

Several criteria were considered in creating a new pump architecture:

- Maximize the transfer efficiency between the diodes and first four slabs

- Obtain an intensity distribution as homogeneous as possible over the $3 \times 5 \mathrm{~cm}$ extracted area

- Minimize the number of components

- Minimize distance between two backplanes

- Minimize multiplexing angle so as to minimize the backplane separation and yet not damage the injection optics

- Keep the overall length as compact as possible

- Reduce diode light scatter back in to the laser chain

- Create a robust and scalable design

\section{Diodes backplane geometry}

The new optimized backplane geometry is shown in Fig. 24. The tile arrangement was chosen to be 5 tiles high by 7 tiles wide since this geometrical arrangement of tiles allows for the optimum transfer efficiency and uniformity in space and angle.

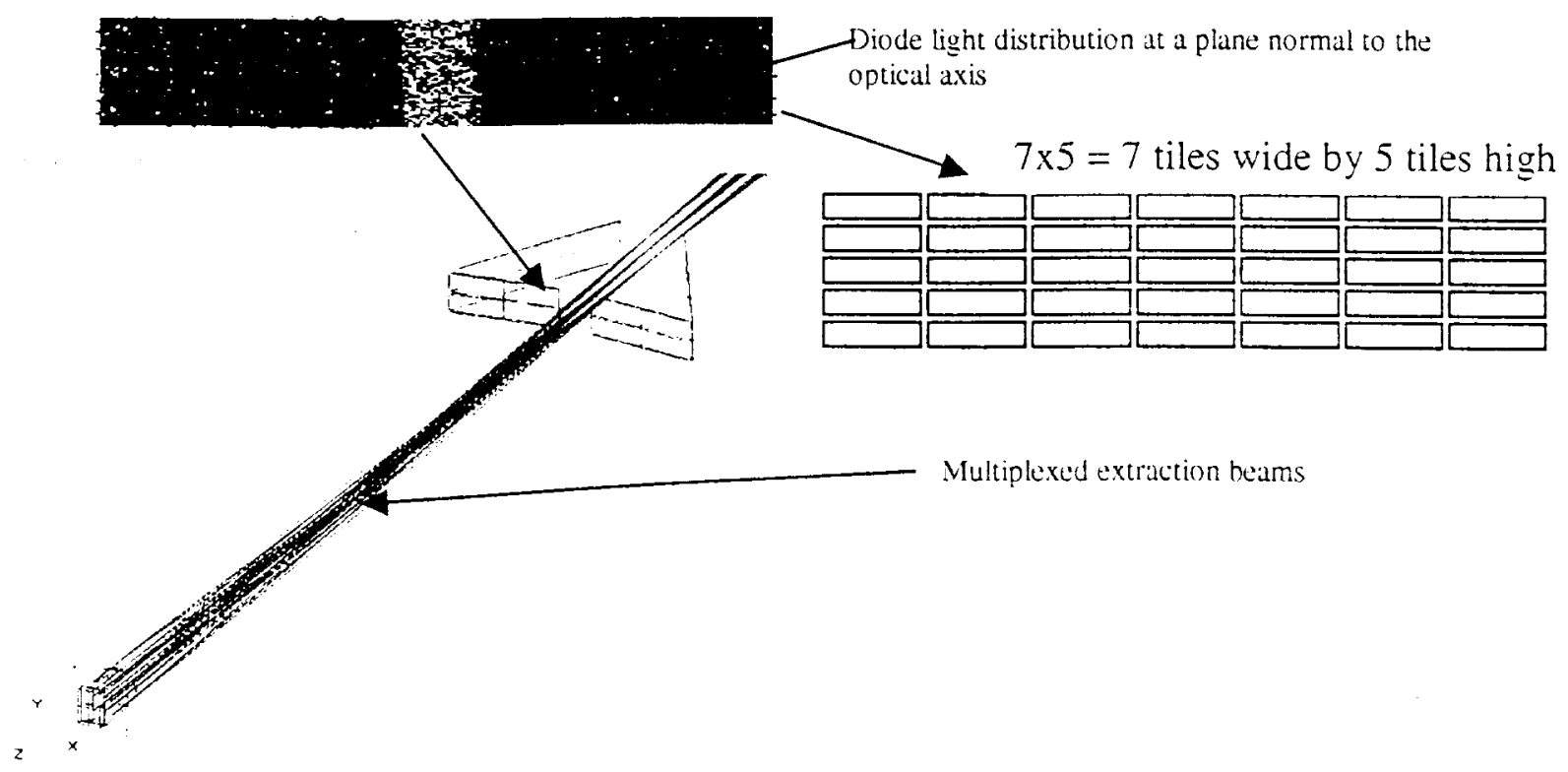

Fig 24. 3D view of one pump delivery system. The four angularly multiplexed beams (blue rays) are shown and can be more easily distinguished at the focal plane level where the beams are geometrically separated. 
A snap shot of a ray trace plane located normal to the ray path is shown in Fig $2+$ where the 14 columns of 5 tiles are can be distinguished. The two rectangular half backplanes consisting of 35 tiles each are separated by 6 to 7 centimeters in order to allow the angularly multiplexed extraction beams to pass through the back. The columns located on the far right and left are very close to the plane and are therefore sharper.

\section{Modeled Transmission Efficiency}

The transfer efficiency is defined as the number of diode light rays that intersect a $3 \times 5$ extracted area in each slab. "Film" planes. which are placed at the front face of every slab, are transparent objects within the Opticad code. to keep track of the rays that pass through a given area. After each computer run. the ray "hits" on each film plane are added up. The details of absorption are not included during the initial optimization runs since only achieving the highest number of hits on each plane is desired. An example of the baseline design and advanced design are compared in Fig. 25. Iterations are performed with different tile geometries to optimize the efficiency. The $6 \times 6$ tile arrangements give the best performance when homogeneity of the pump light distribution is considered as well.

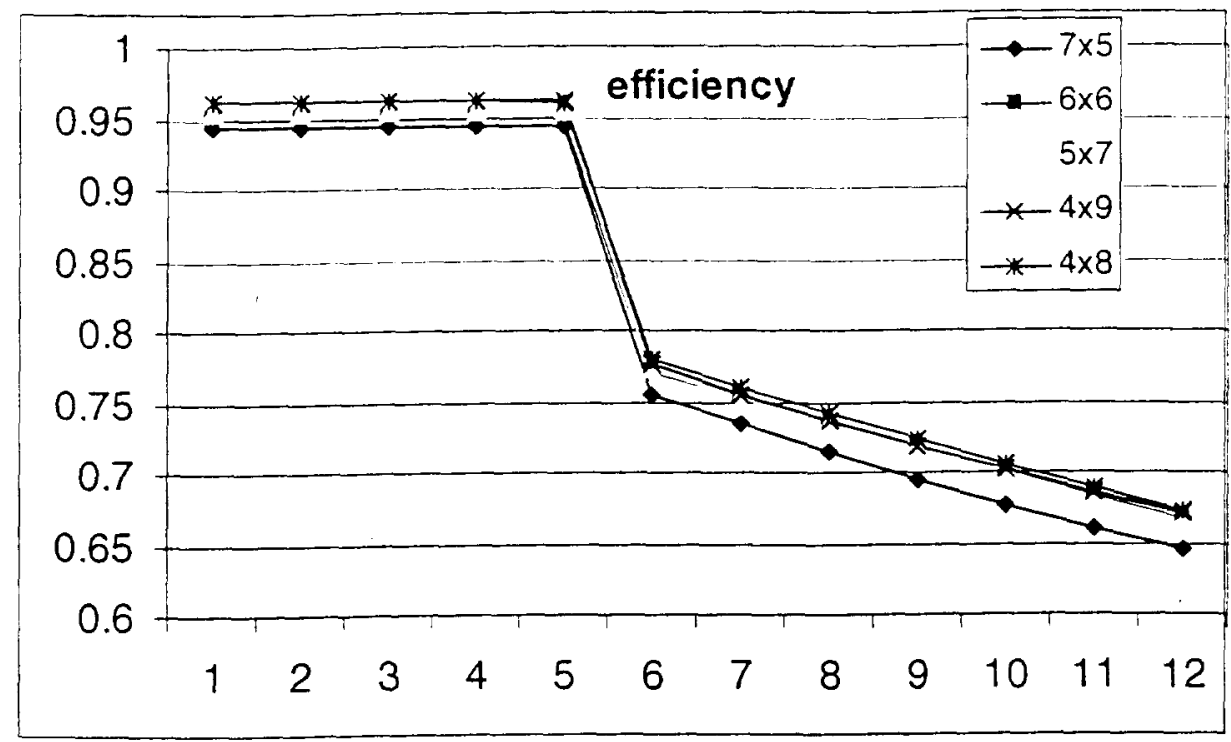

Fig. 25 Transport efficiency through the homogenizer and slabs as a function of the tile arrangement. Points 1-5 are within the homogenizer. Points 6-12 are within the 7 individual slabs.

\section{Pumping homogeneity}

The next step in the optimization involves creating a uniform intensity distribution in the slabs. The length of the homogenizer is varied to obtain the best results. The pump light distribution at each of the seven slabs. including the exit plane of the homogenizer is shown in Fig. 26. The fill factor and uniformity is very high in the first slab. However. as the beam propagates further into the amplifier. the high angle light is stripped and leads to a narrower and more non-uniform spatial distribution of light. Horizontal lineouts of the first and last slab are shown in Fig. $27 \mathrm{a}$ and b. As the beam propagates further in to the amplifier the intensity roll-off becomes more pronounced. The dual ended pumping scheme allows for some compensation of this roll off since most of the light is predominately absorbed in the first four slabs on each side. 


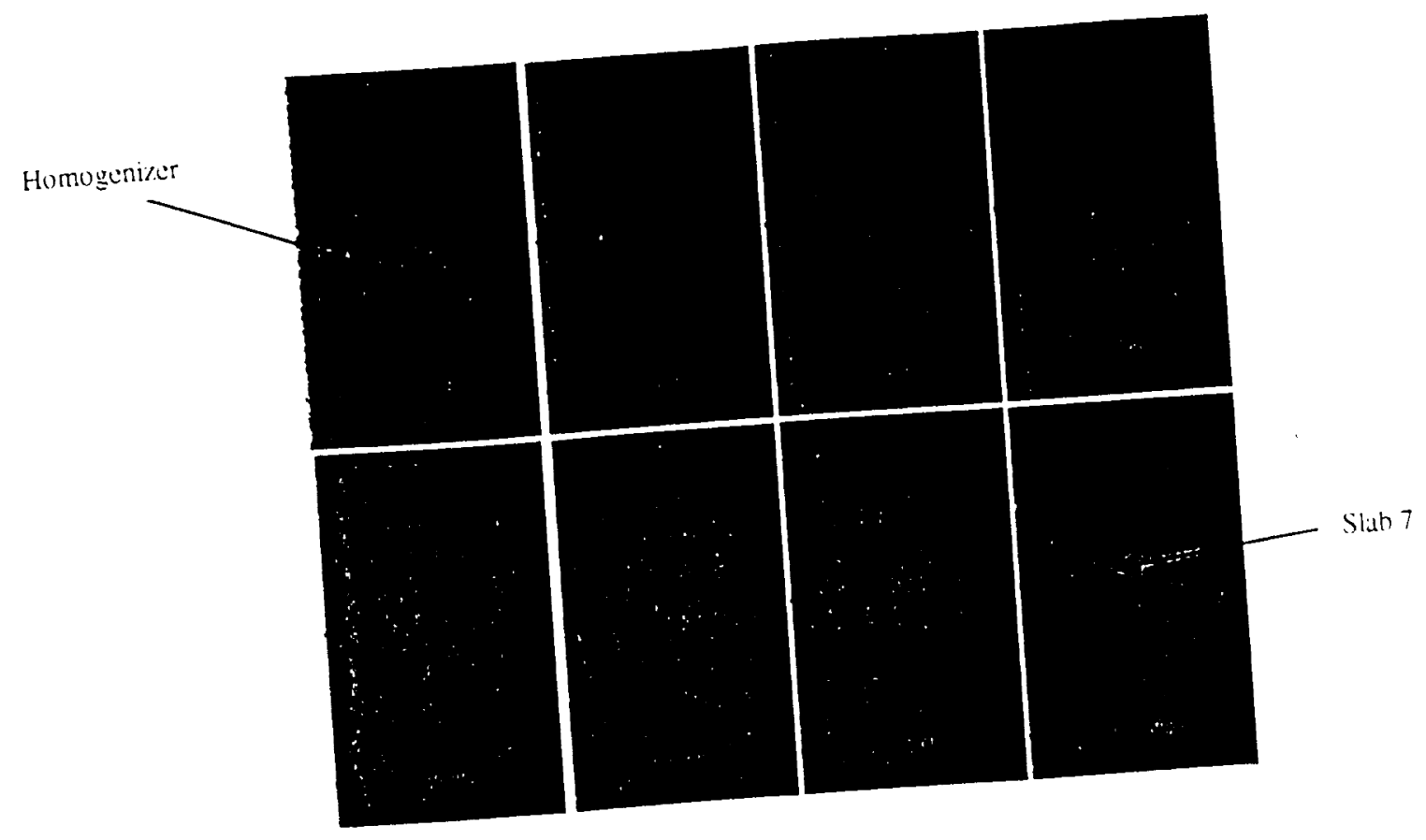

Fig. 26 Single-sided pump distributions without absorption included.

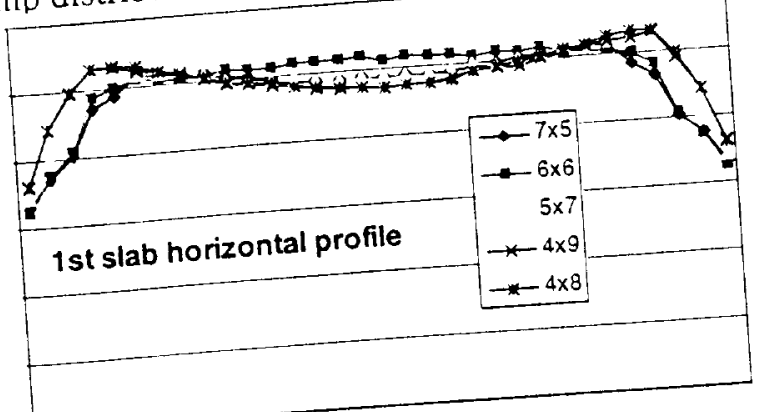

Fig 27a Horizontal lineouts in the first slab to compare different tile arrangements.

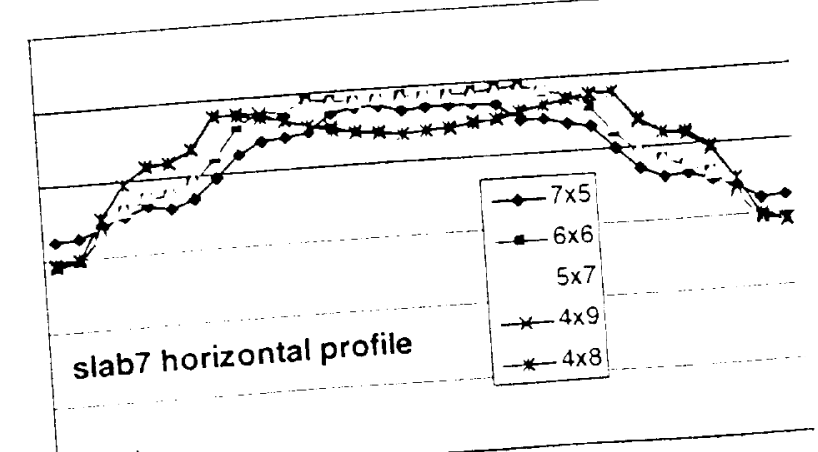

Fig $27 \mathrm{~b}$ Horizontal lineouts in the last slab to compare different tile arrangements. 
The tinal design employs a $6 \times 6$ tile arrangement with a $40 \mathrm{~cm}$ long homogenizer. Improvements to the overall performance can be achieve with either higher power diode bars, so that less tiles are required or a smaller divergence per tile. Future diode designs are likely to employ both of these improvements. 


\section{System Design}

\section{Angular multiplexing}

The t-pass angular multiplexing scheme used in Mercury follows a similar concept employed on the French laser system (Laser Megajoule). This multipass architecture scheme avoids the use of expensive. large aperture. high average power optical switches. In Mercury. after two passes through laser system. the beam is turned around with tuming mirrors near the injection optic and reinjected into the laser for two final passes.

In the original baseline design. the injection hardware. diode pump hardware and optical telescopes for the extraction beam were independent. In the new design. all of the hardware components are intimately coupled. The relay telescopes have been designed to be slightly longer in order to allow the extraction beam to focus outside of the hollow duct enclosure (see Fig 28). This is required since the baftles or large pinholes placed at the focal plane would intertere with the transport of the diode light to the slabs. In order to keep the split backplanes as close as possible. one of the telescope lenses is placed next to the amplifier head. This creates a smaller focal beam pattern near the backplane. The lens placement next to the amplifier head also helps mitigate optical damage as discussed below. Another hidden advantage is that the angular separation for the multiplexed beams becomes nonsymmetrical about the focal plane. which allows for the insertion of a larger injection mirror (see near focal plane in Fig 28).

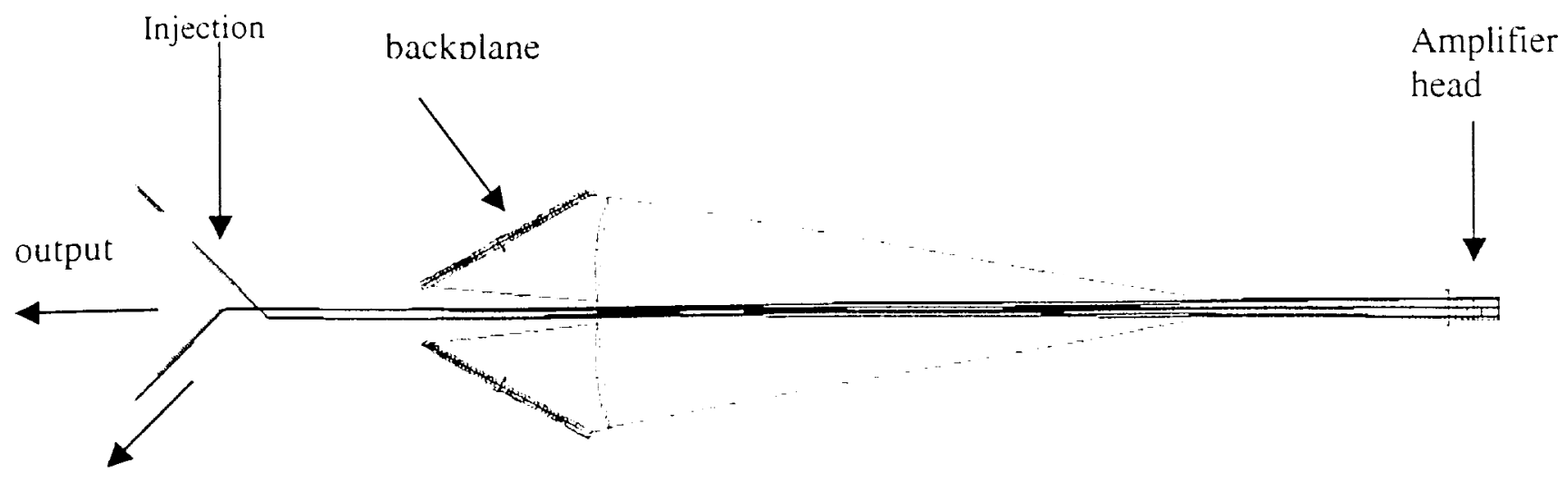

Towards

reverser

Fig. 28 Top view showing injection scheme.

\section{Damage issues}

We are in the process of completing a thorough analysis of potential damage concerns with the advanced design. The primary issues involve ghost foci from telescope lenses, pencil beams, etaloning effects from the nearly parallel amplifier slabs. diode stray light. ASE. and B-integral. The Opticad code is being used to mock up the entire laser system to help assess these concerns and help reduce the risk of damage.

\section{Proposed system arrangement}

The overall layout of the advanced design is shown in Fig 29 along with several key design considerations. The near term goals are to resolve the remaining damage concerns and begin building hardware for full system demonstrations. 


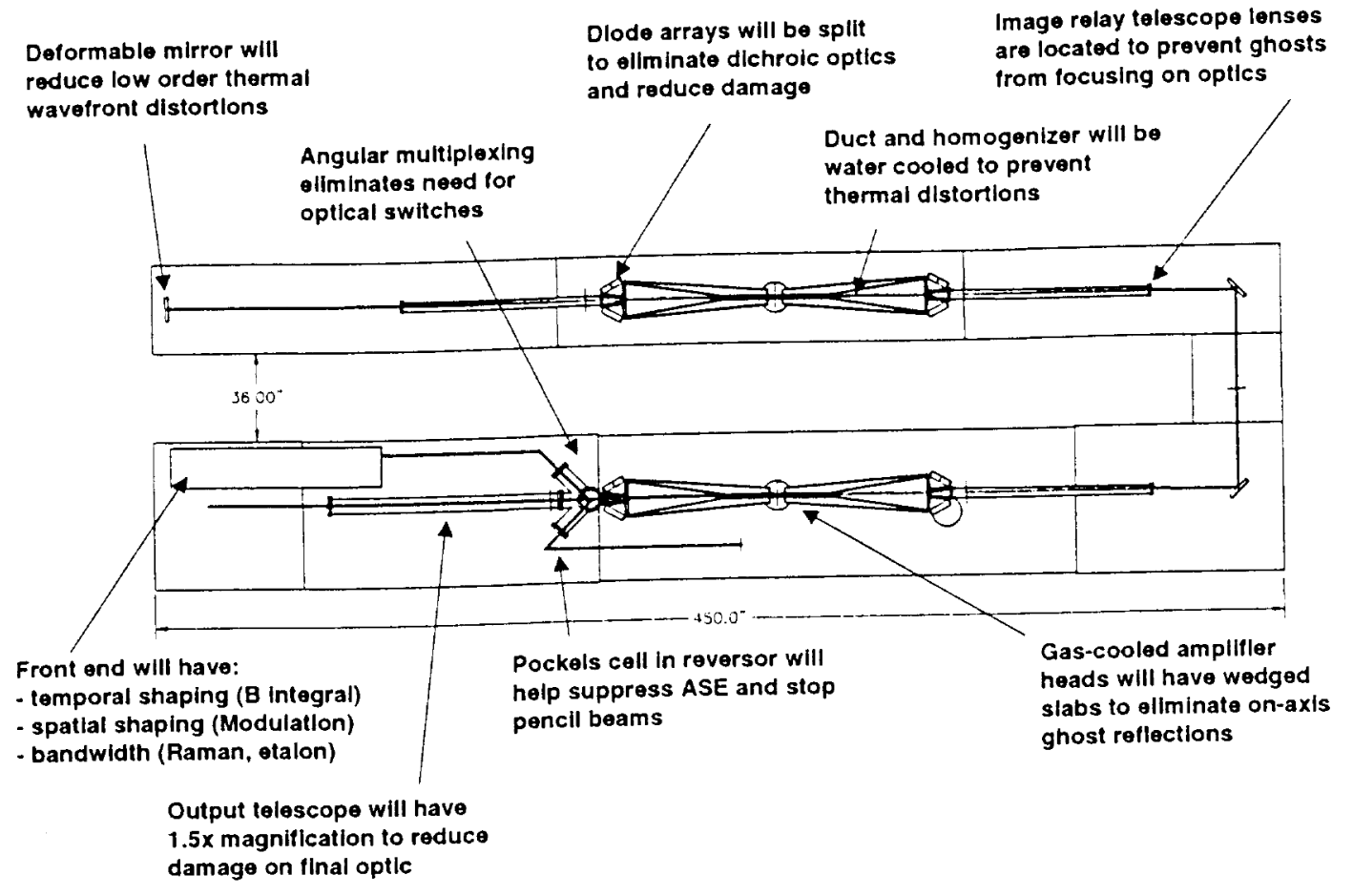

Fig. 29 The advanced optical layout along with key elements highlighted. 


\section{Czochralski Crystal Growth}

\section{A. Growth Methology}

The goals of the crystal growth effort are to produce high quality $\mathrm{Yb}: \mathrm{S}$-FAP $\left[\mathrm{Yb}^{3+}: \mathrm{Sr}_{5}\left(\mathrm{PO}_{4}\right)_{3} \mathrm{~F}\right]$ crystals: this involves developing an outside company resource for the growth of full size crystals and assessing the feasibility of bonding smaller crystals together to produce full aperture crystals with no compromise to the optical quality. The Mercury design requires crystalline slabs of dimension $4 \times 6 \times$ $0.75 \mathrm{~cm}$. Fig. 30 , which will be potted into vanes and mounted into two amplifier heads: seven slabs will be required for each head. The growth of full aperture crystals has been a challenge due to a number of defects. including: cloudiness in as-grown boules. bubble core defects. grain boundaries, anomalous absorption. and cracking in larger diameter boules $>4.0 \mathrm{~cm}$. Over the last two years, a third Czochralski crystal growth station has been assembled to accelerate the growth effort. chillers have been added to control cooling water temperatures to the power supplies thus regulating the heat load. and a motor generator was installed to damp out fluctuations in the incoming power to the power supplies.

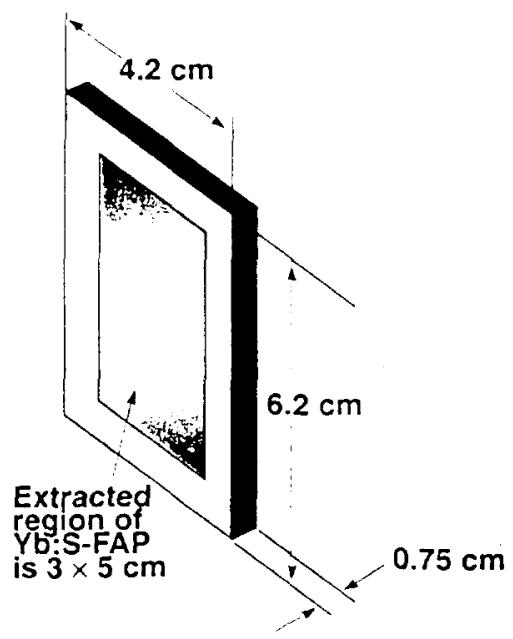

Fig. 30. Schematic of the slab dimensions required for the Mercury design.

Significant progress has been made in understanding the growth characteristics and defect chemistry of Yb:S-FAP crystals. In an effort to understand the defect issues in Yb:S-FAP and find a feasible growth technique for producing high optical quality crystals, a collaborative research effort has been put forth within LLNL and among experts in the growth of high temperature crystals at commercial crystal growth companies: (1) Dr. Robert C. Morris (formerly of Allied Corp.). a world-renowned leader in solving defect problems in optical crystals, has been participating as a consultant in the growth of Yb:S-FAP. (2) Robert S. Feigelson of Stanford University has provided a great deal of technical insight and expertise. (3) Ralph Hutchinson at Scientific Materials (Bozeman. MT) has grown several crystals in a "high thermal gradient" furnace regime (will be described in more detail below). Most recently. LittonAirtron. Synoptics Group has been engaged and have shown a great deal of interest in the development of $\mathrm{Yb}: \mathrm{S}-\mathrm{FAP}$ as an industrial laser crystal. They initially explored the growth of this material with their own internal research funding thus far: however, they were recently awarded a contract by LLNL to begin growing crystals. One advantage of working with Litton is that they have a proven facility in place for the growth of high quality crystals of the large dimension needed for Mercury and next generation lasers. 


\section{Growth Process}

Crystals of Yb:S-FAP are grown by using the Czochralski method in three growth stations one of which is shown in Fig. 31 a along with a schematic of the furnace design and basic growth parameters. Fig. $3 \mathrm{lb}$. The growth station consists of a Czochralski crystal puller. a $25 \mathrm{kWatt}$ induction power supply. and a computer program that is used for automatic diameter control (ADC) of the crystal. Automatic diameter control is achieved by weighing the crystal as it is growing and calculating the mass gain per unit time (grams/hour or $\mathrm{g} / \mathrm{h}$ ): is utilized so that the diameter of the crystal is constant at a given pull rate. A 4 " diameter by + " tall Iridium crucible was chosen to contain the melt because it does not chemically react with Yb:S-FAP and has a high melting temperature. Zirconia is the main insulation component in the furnace as shown in Fig. $3 \mathrm{lb}$. The Iridium crucible and melt therein is heated by inductive coupling of the crucible and copper induction coil.
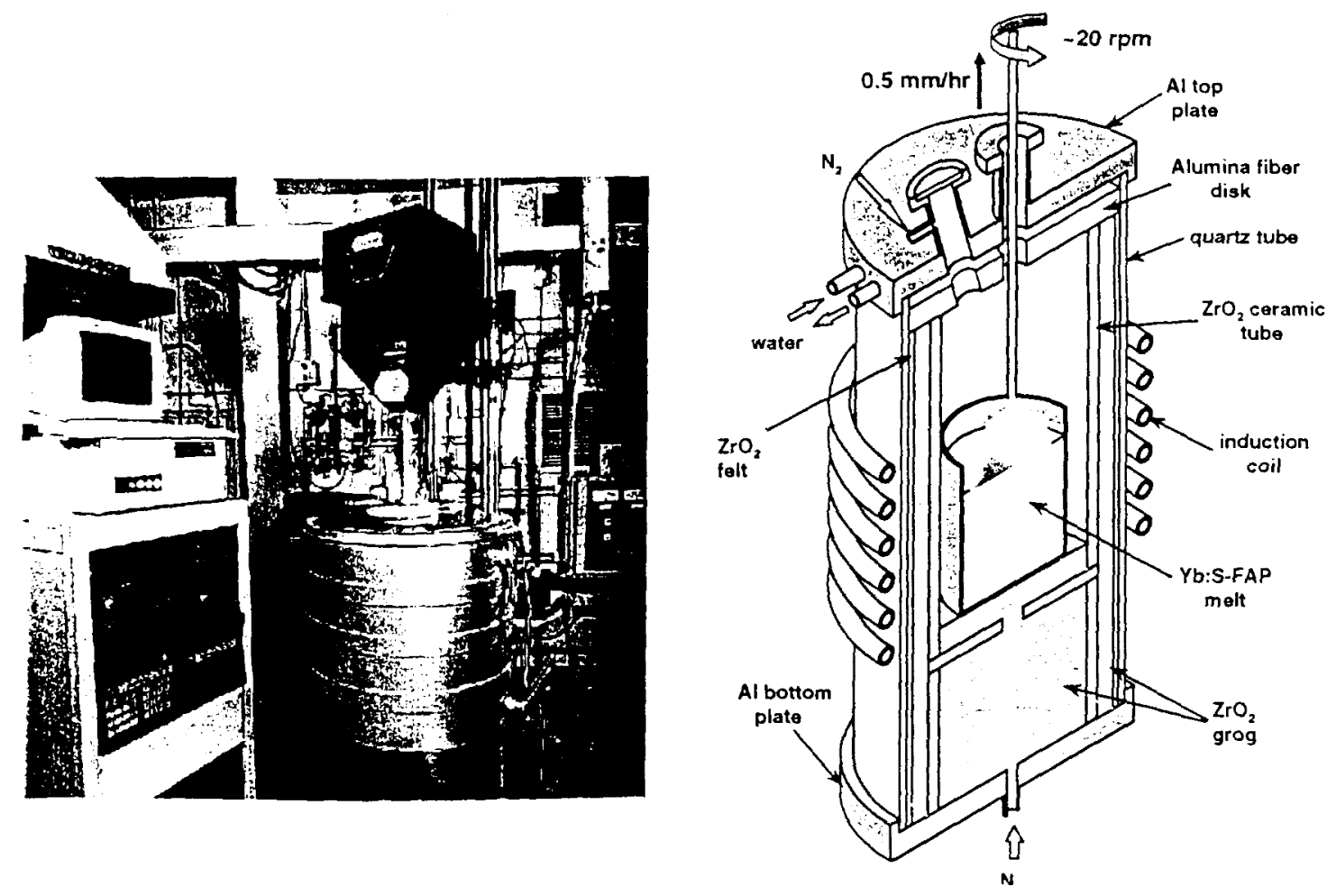

Fig. 31. (a) Picture of the Czochralski crystal growth station used for Yb:S-FAP growth. (b) Schematic of the fumace design with approximate growth parameters.

A melt is loaded into the crucible in two steps: a decomposition step followed by the addition $\mathrm{SrF}_{2}$ to the melt. The basic chemical formulas for the two step reaction are described below (Eqns $1 \& 2$ ) where 1 atomic percent $(\mathrm{At} \%)$ or 1.17 weight percent (wt\%) Yb doping is used. Specifically, the strontium hydrogen phosphate. carbonate. and dopant are loaded into the crucible and allowed to fully decompose overnight forming strontium phosphate. The pre-decomposition reaction prevents the $\mathrm{SrF}_{2}$ from oxidizing to $\mathrm{SrO}$ thereby removing fluorine from the melt and creating defects in the boule during growth. An inert nitrogen atmosphere is also used for this reason as well as for preventing oxidation of 
the Iridium crucible. There is a loss of $\mathrm{SrF}$, due to vaporization at the melting temperature of $1800^{\circ} \mathrm{C}$. therefore excess fluoride is added to the starting melt. The amount of excess will be discussed in more detail later in the defect section of this report since it has a significant effect on the cloudiness in as grown boules. The melt is allowed to equilibrate for $2-+$ hours before seeding. The chemical process maybe described as:

$$
\begin{aligned}
& 6 \mathrm{SrHPO}_{4}+2.90 \mathrm{SrCO}_{1}+0.10 \mathrm{Yb}_{2} \mathrm{O}_{3} \rightarrow 3 \mathrm{Sr}_{2} \cdot \mathrm{n}_{7} \mathrm{Yb}_{1,1033}\left(\mathrm{PO}_{4}\right)_{2}+2.90 \mathrm{CO}_{2}+3 \mathrm{H}_{2} \mathrm{O} \\
& 3 \mathrm{Sr}_{2}, y_{7} \mathrm{Yb}_{1,13}\left(\mathrm{PO}_{4}\right)_{2}+\mathrm{SrF}_{2} \rightarrow 2 \mathrm{Sr}_{4}, \mathrm{Yb}_{0.015}\left(\mathrm{PO}_{4}\right)_{3} \mathrm{~F}
\end{aligned}
$$

The time required for the current growth geometry of Yb:S-FAP crystals of suitable size to harvest slabs is approximately 15 days and encompasses a series of four steps that are critical to producing high quality material. Fig. 33. The first step requires that the seed be extended to grow out the grain boundaries from the crystal. The seed extension is necked in to $2-3 \mathrm{~mm}$ diameter to aid in growing the defects out toward the sides of the crystal where they will terminate. The cone section of the boule is the next critical step since the overall growth rates tend to be much higher than in other parts of the growth process and defects can form. For this reason the cone angle is kept very small or basically. the diameter of the boule is slowly increased to the full diameter. In the cylinder section of the boule. it is essential that stable growth conditions be maintained to preserve the defect free nature of the crystal from which slabs will be cut. Finally. the crystal is necked in at the bottom and allowed to cool in the melt to decrease the thermal gradients through the boule during cooling and reduce cracking. A pull rate of 0.5 $\mathrm{mm} / \mathrm{hr}$ is currently used since higher rates have shown an increase in the magnitude of the defects. The rotation rate is typically $\sim 20 \mathrm{cpm}$ to provide a convex melt-interface shape that is stable and does not introduce unwanted fluctuations that can perturb the growth. This specific growth technique has significantly reduced the number of defects and yielded high quality material that nearly meets the Mercury specifications. A 4" diameter by 4" tall iridium crucible was chosen to contain the melt because it does not chemically react with Yb:S-FAP and has a high melting temperature. Zirconia is the main insulation component in the furnace as shown in Fig. $3 \mathrm{lb}$. The iridium crucible and melt therein is heated by inductive coupling of the crucible by the copper induction coil.

Herein. a summary of each of the defects will be described along with methods for improving or eliminating them. Of the five prevalent defect issues, solutions for each have been formulated and put into practice: however, the bubble core is the remaining issue. Our strategy is to either eliminate it or concentrate it to the center of the boule so that usable material can be cut from the areas around it. The latter technique has previously been used by Litton-Synoptics in their high-temperature growth of large crystals such as YAG and GGG, which with have concentrated core in the boule centers.

\section{Growth Improvements}

Over the last year, several improvements have been implemented to increase the ability to produce high optical quality Yb:S-FAP with reproducibility. The installation of a second growth station has allowed for an increased number of erystal growth experiments to be performed so that the parameter space can be explored in a shorter period of time. Water recirculation chillers have been added to the power supplies to damp out periodic fluctuations in the cooling water temperature. which caused ridges on the crystal due to instabilities in the growth interface. This instability is a source of defect formation and therefore a critical variable to control. In addition, a motor generator is being added for power conditioning on the incoming supply line that feeds into the induction power supplies (Fig 34). The power stability will increase interface control and decrease the number of crystal growth failures from power surges and drops. A third furnace has been dedicated to decomposing starting materials in an effort to better control the exact ratios of the melt components. It is believed that the root of most of the defect 
structures is related to issues with composition variations. Each of these improvements will provide better control of the variables in the crystal growth and help to increase reproducibility from growth to growth.

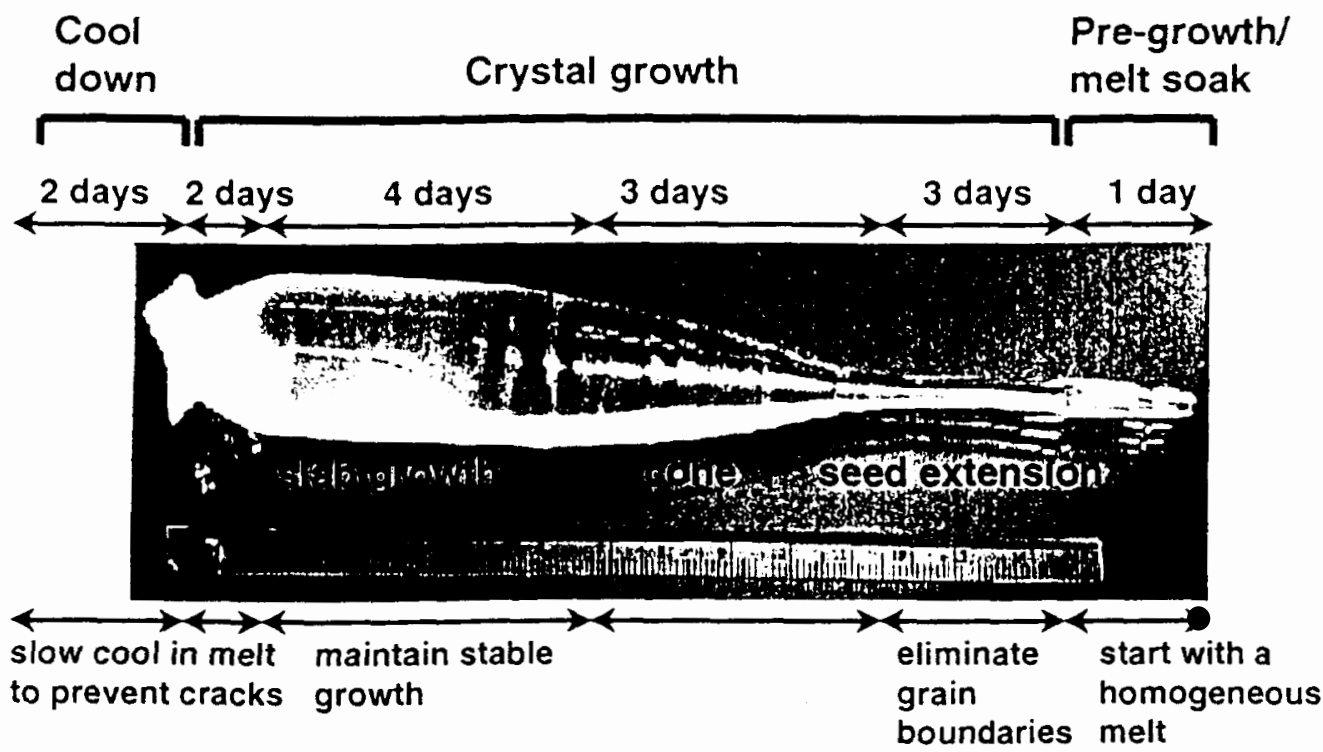

Fig. 33. Current technique for the growth of Yb:S-FAP crystals
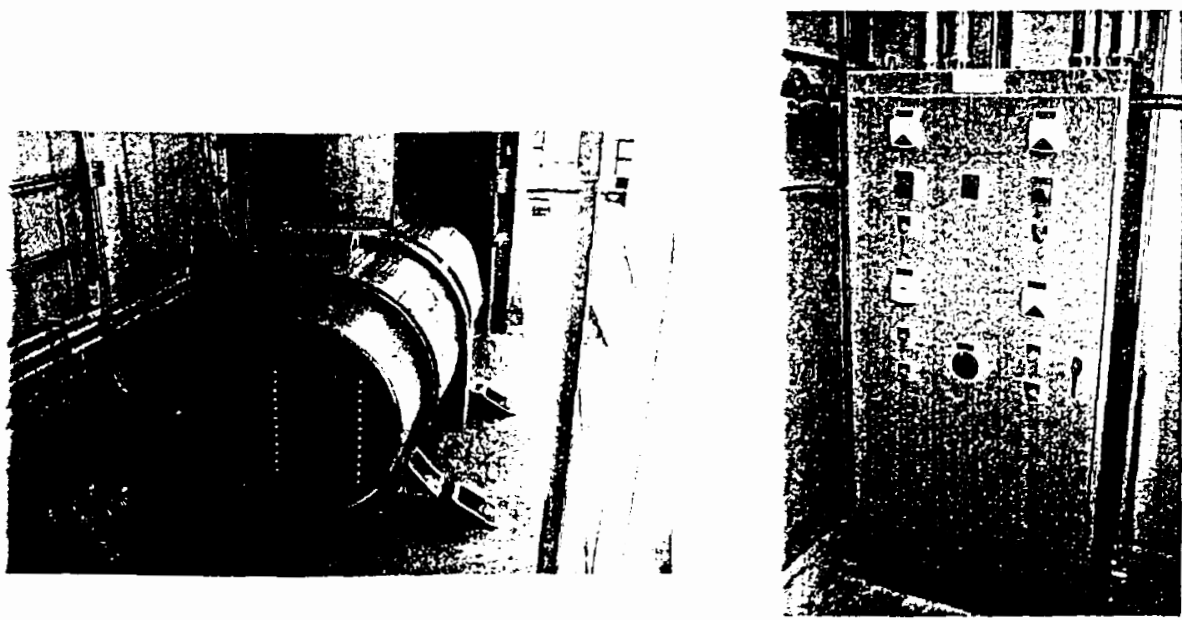

Fig. 34. Pictures of the motor generator and control panel used for power stabilization.

\section{B. Defect Chemistry}

Yb:S-FAP crystals can show a number of defect structures that occur in various forms. In particular, cloudiness, an anomalous absorption, bubble core defects, grain boundaries, and cracking in larger diameter boules with a significant number of bubbles. Each of these defect structures has been 
studied in considerable detail and solutions to reduce and /or eliminate them have been implemented as outlined below in Table 2 .

Table 2. Summary of defect issues and the solutions.

\begin{tabular}{|c|c|c|c|}
\hline Issue & $\begin{array}{c}\text { Physical } \\
\text { Understanding }\end{array}$ & Action/Plan & Status \\
\hline Cracking & $\begin{array}{l}\text { Results from high thermal } \\
\text { gradients and internal defects }\end{array}$ & $\begin{array}{l}\text { 1) cool crystals in the melt } \\
\text { 2) reduce core defects }\end{array}$ & Acceptable \\
\hline Cloudiness & $\begin{array}{l}\text { Second phase precipitation on } \\
\text { line defects }\end{array}$ & $\begin{array}{l}\text { 1) Annealing } \\
\text { 2) excess SrF, }\end{array}$ & Resolved \\
\hline $\begin{array}{l}\text { Anomalous } \\
\text { absorption }\end{array}$ & $\mathrm{Yb}^{3+}$ in a different type of site & $\begin{array}{l}\text { 1) grow } 45^{\circ} \text { to } c \text { orientation } \\
\text { or along c axis }\end{array}$ & Resolved \\
\hline Grain boundaries & $\begin{array}{l}\text { Planar groups of dislocations } \\
\text { that propagate from the seed or } \\
\text { originate at the core }\end{array}$ & $\begin{array}{l}\text { 1) seed extensions } \\
\text { 2) eliminate core defects } \\
\text { 3) stabilize growth interface }\end{array}$ & $\begin{array}{l}\text { Nearly } \\
\text { Resolved }\end{array}$ \\
\hline Core defects & $\begin{array}{l}\text { Caused by constitutional } \\
\text { supercooling }\end{array}$ & $\begin{array}{l}\text { 1) stabilize growth interface } \\
\text { (chillers, motor generator) } \\
\text { 2) minimize abrupt changes } \\
\text { in diameter } \\
\text { 3) Concentrate core in center } \\
\text { with high thermal gradient or } \\
\text { grow boules } 2.3 \mathrm{~cm} \text { in size }\end{array}$ & $\begin{array}{c}\text { Under } \\
\text { Investigation }\end{array}$ \\
\hline Size & $\begin{array}{l}\text { Current growth capability limits } \\
\text { crystal diameter to } \sim 4-5 \mathrm{~cm}\end{array}$ & $\begin{array}{l}\text { 1) diffusion bond two pieces } \\
\text { together to make a full size } \\
\text { slab }\end{array}$ & Resolved \\
\hline
\end{tabular}

\section{Cracking}

Cracking in the crystals has been almost completely eliminated with improved growth conditions (Fig. 35a). Major causes of cracking are high thermal gradients in the crystal during the cooling phase and stress from internal defects such as core. The crystals are now cooled in the melt to reduce the thermal gradients during the cool down process. This keeps the boule in contact with the thermal bulk of the melt and the heat transferred to the crystal lowers the gradient from top to bottom reducing the induced stresses created by a high thermal gradient that often leads to cracking. In addition, reduction of internal defects, such as core, will greatly reduce cracking in the crystals.

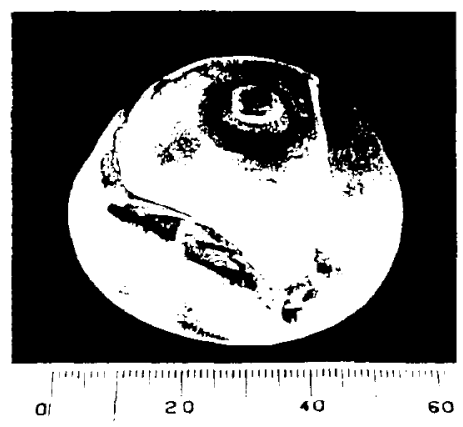

Fig. 35a Previous large diameter growth conditions led to cracking. 


\section{Cloudiness}

Cloudiness in as-grown boules is believed to be associated with precipitation of a second phase onto line defects in the crystal lattice (Fig. $35 \mathrm{~b}$ ). It can be eliminated by simply adding $33 \mathrm{~mol} \%$ excess $\mathrm{SrF}$ : (a component making up the S-FAP structure) to the initial melt composition. Completion of a partial phase diagram for S-FAP. currently underway at NIST (National Institute of Standards and Technology). should provide insight into these affects of composition on crystal quality as well as determining the congruent melting composition. The cloudiness can also be eliminated a second way, by holding the boule above the melt for several days and annealing away the microscopic defects.

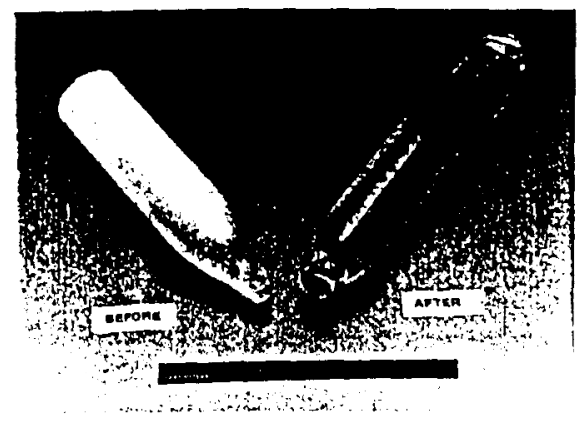

Fig. 35b Annealed and un-annealed Yb:S-FAP boules.

\section{Anomalous absorption}

Recent absorption spectra have shown the presence of a broad anomalous absorption feature at $975 \mathrm{~nm}$ that is attributed to $\mathrm{Yb}^{3+}$ in a different environment than gives rise to sharp absorption features at $\sim 900 \mathrm{~nm}$. There are two distinct $\mathrm{Sr}$ sites in the $\mathrm{Sr}_{5}\left(\mathrm{PO}_{4}\right)_{3} \mathrm{~F}$ structure; the $\mathrm{Sr}_{1}$ site is nine fold coordinated to $\mathrm{O}\left(\mathrm{Sr}_{\mathrm{l}} \mathrm{O}_{9}\right)$ and the $\mathrm{Sr}_{\mathrm{I}}$ site is bonded to six oxygens and one fluorine $\left(\mathrm{Sr}_{\mathrm{d}} \mathrm{O}_{6} \mathrm{~F}\right)$. $\mathrm{Yb}^{3+}$ on the $\mathrm{Sr}_{\mathrm{II}}$ site is charge compensated by replacing the $\mathrm{F}$ with an $\mathrm{O}^{2-}$ and this $\mathrm{Yb}-\mathrm{O}$ bond is what gives rise to specific vibrational modes that result in the desired laser characteristics for the material ${ }^{12}$. The anomalous absorption is believed to be $\mathrm{Yb}^{3+}$ on the $\mathrm{Sr}_{1} \mathrm{O}_{9}$ site, which has no straightforward internal charge compensation mechanism. Recent results of a crystal grown at Scientific Materials ${ }^{13}$ indicate that it may be possible to eliminate the presence of the anomalous absorption by growing crystals along a direction that is $45^{\circ}$ to the $c$-axis. We have recently discovered that the growth along the c-axis also reproducibly eliminates the anomalous absorption.

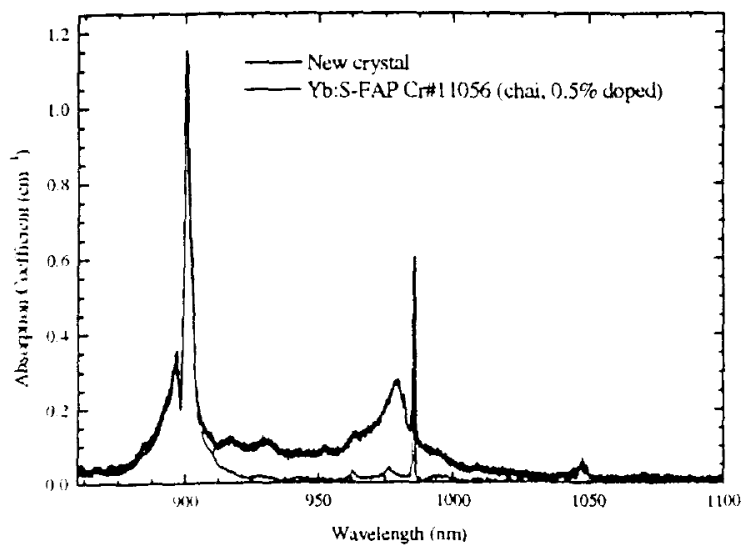

Fig. 35c The broad absorption peak is due to $\mathrm{Yb}^{3+}$ in an unwanted site. 


\section{Grain Boundaries}

Another critical issue associated with Yb:S-FAP crystals if that of low-angle grain boundaries. (Fig. 35d). This phenomenon appears as slight changes in the refractive index of the crystal or waves running through the crystal with a "columnar-type" orientation perpendicular to the $c$-axis. These boundaries are formed by propagation from the seed to the crystal and strain-induced detormations caused by an unstable growth intertace. Reducing the number of grain boundaries has required using "defectfree" seeds and careful consideration of the growth rate and intertace shape to stabilize growth and prevent strain in the crystals. We have successfully developed a method to remove the grain boundaries from the seed by "necking down" the crystal during the growth to propagate the defects out of the crystal. Also. stabilization of the growth interface has proven effective in preventing new boundaries from forming and has been dramatically improved with the closed-cycle water chiller and the installation of a motor generator for power conditioning.

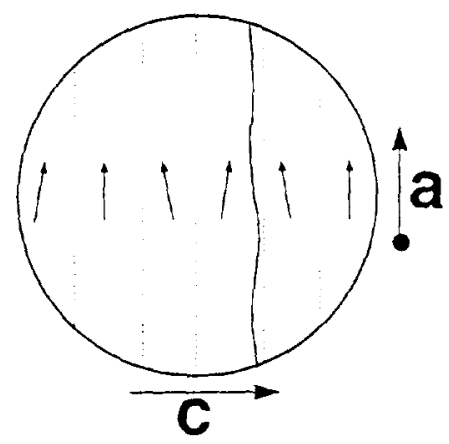

Fig. 35d Grain boundaries appear to eye as wavy lines due to the variation in index of refraction.

\section{Bubble core}

The bubble core (Fig. 35e) is the main focus of continuing research, since methods for reducing or eliminating all other defects have proven successful. Bubble core defects are found to be attributed to an unstable growth interface between the crystal and melt causing pockets of trapped liquid which form bubble vacancies upon solidification and is attributed to constitutional supercooling ${ }^{14}$.

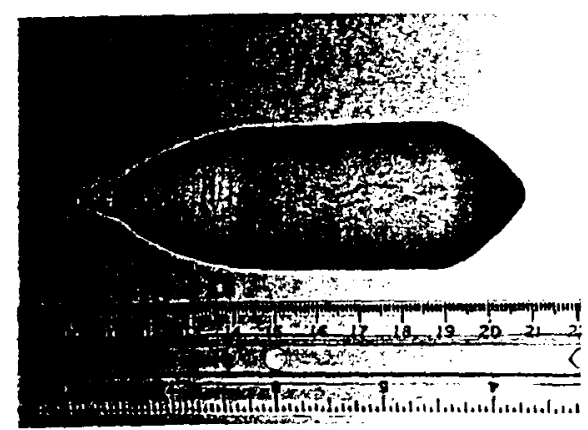

Fig. $35 e$ Core defects can be seen along the growth axis of the boule

The composition of the liquid pockets is a product of the rejected components in the diffusion boundary layer between the crystal-melt interface. Scanning electron microscopy in conjunction with Xray analysis (SEM-EDS) have indicated that $\mathrm{Yb}_{2} \mathrm{O}_{3}$ and $\mathrm{Sr}_{3}\left(\mathrm{PO}_{4}\right)_{2}$ are associated with the bubble vacancies, Fig. 36. These are natural by-products from a deficiency of $\mathrm{SrF}_{2}$ at the growth interface caused by its evaporative loss. Slabs cut from a recently grown crystal are shown in Fig. 38. where the $c$-axis is along the long direction of each slab. cut from top-to-bottom (a-to-d) of a $45^{\circ}$ to $c$-axis crystal. In these cut slabs. it has been found that the bubble core tends to propagate along the $c$-axis in the crystals beginning at a diameter of about $2.5 \mathrm{~cm}$ and then increasing as the crystals approach full diameter $(\sim-.5$ 


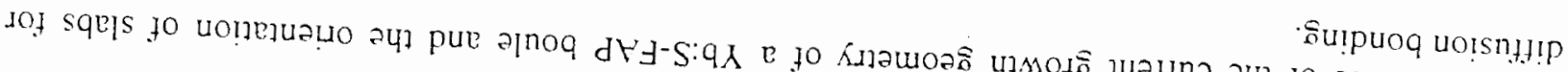

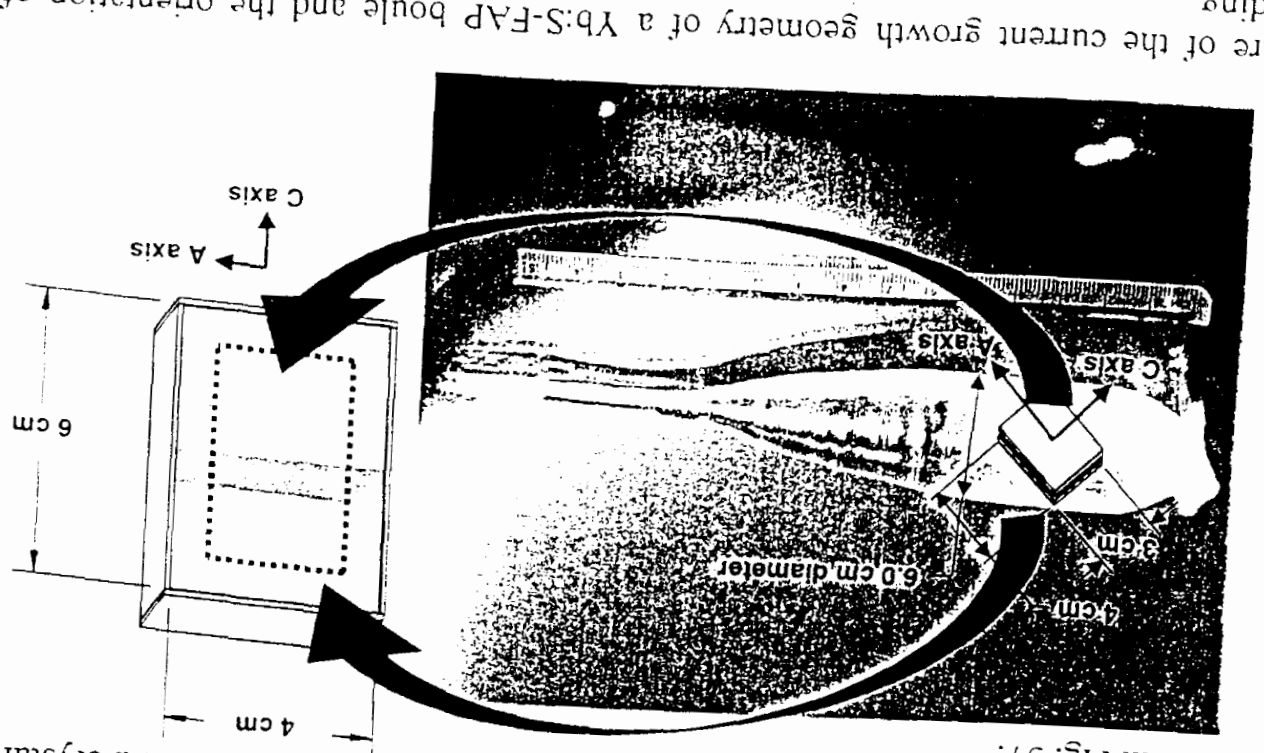

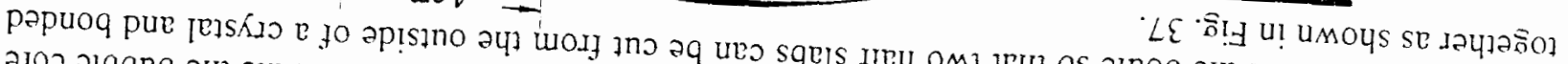

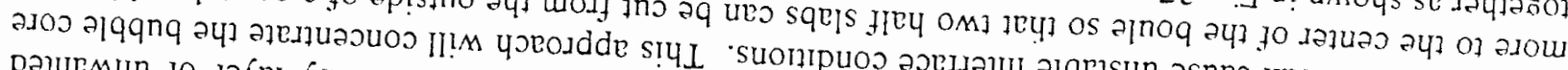

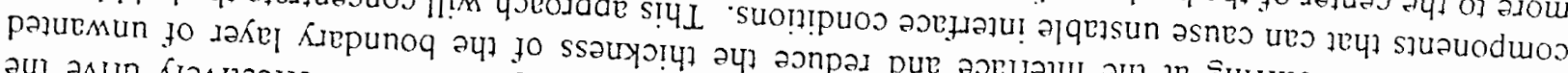

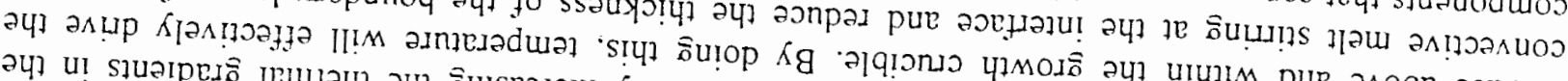

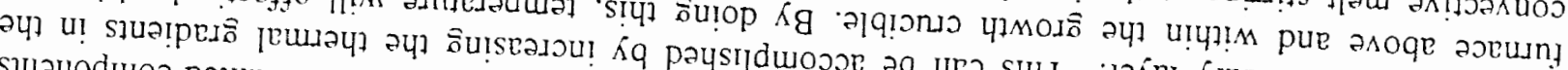

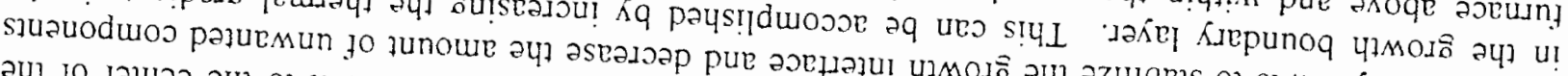

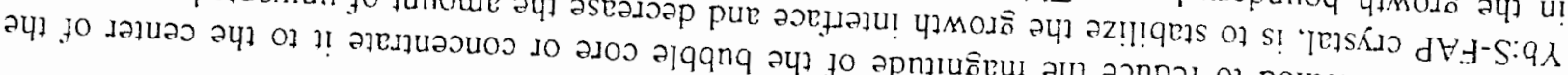

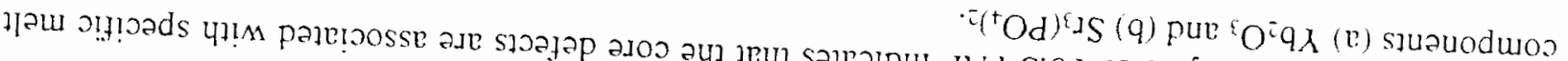
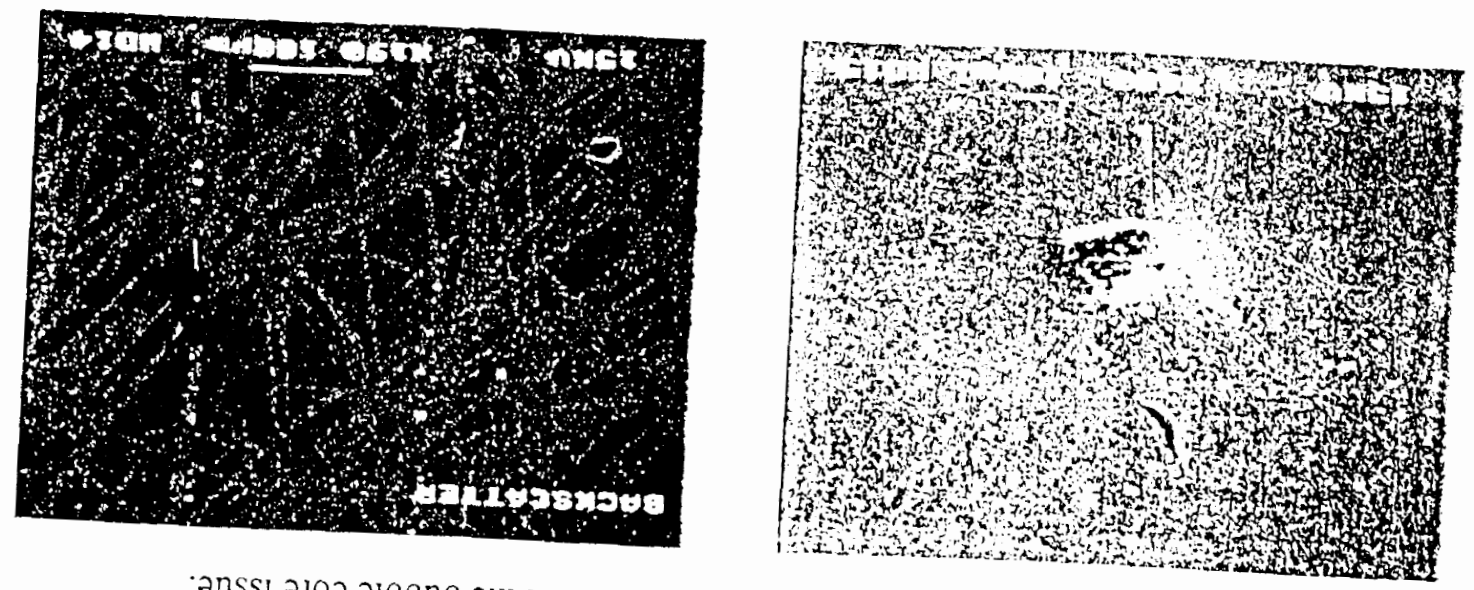

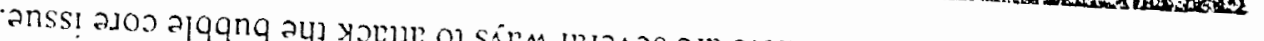

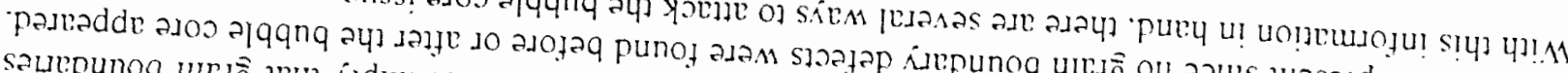

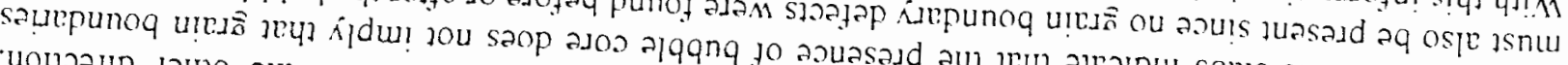

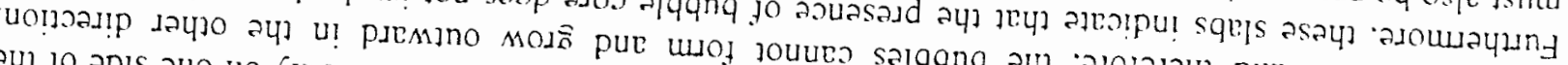

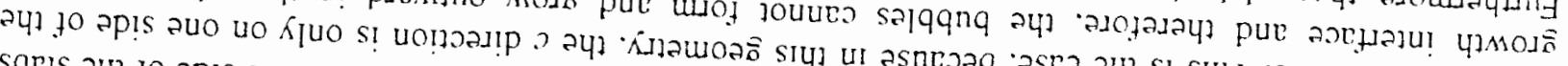

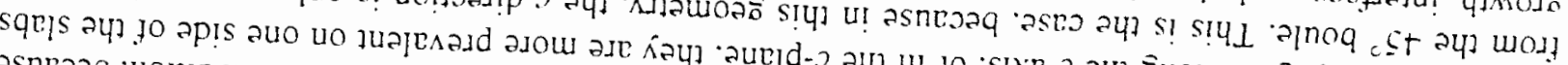

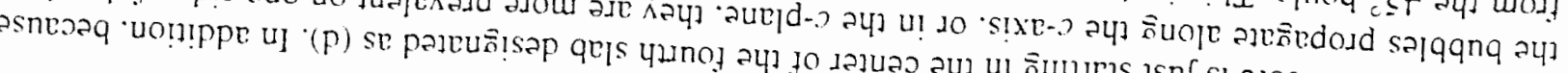


A second approach for attacking the bubble core issue is to grow smaller diameter boules of about $2.5 \mathrm{~cm}$. which is smaller than the diameter where the bubbles begin to form. see Fig.38. From the smaller diameter boules. quarter slabs could then be cut and bonded together. The ability to bond a 4 -piece slab has recently been demonstrated by ONYX Optics. for slightly sub-scale pieces than what will be necessary for $a+x 6 \mathrm{~cm}$ slab for Mercury. Fig. 39. It should be noted that the pieces that make up this slab were trom previously cut crystals and are not precisely oriented therefore bond lines are observable (not seen in picture). This slab was just recently received and measurements will determine if this is a viable approach to obtaining a full size slab for Mercury. However. the ability to bond four pieces together is encouraging for a near term solution to the crystal growth issues for Yb:S-FAP.

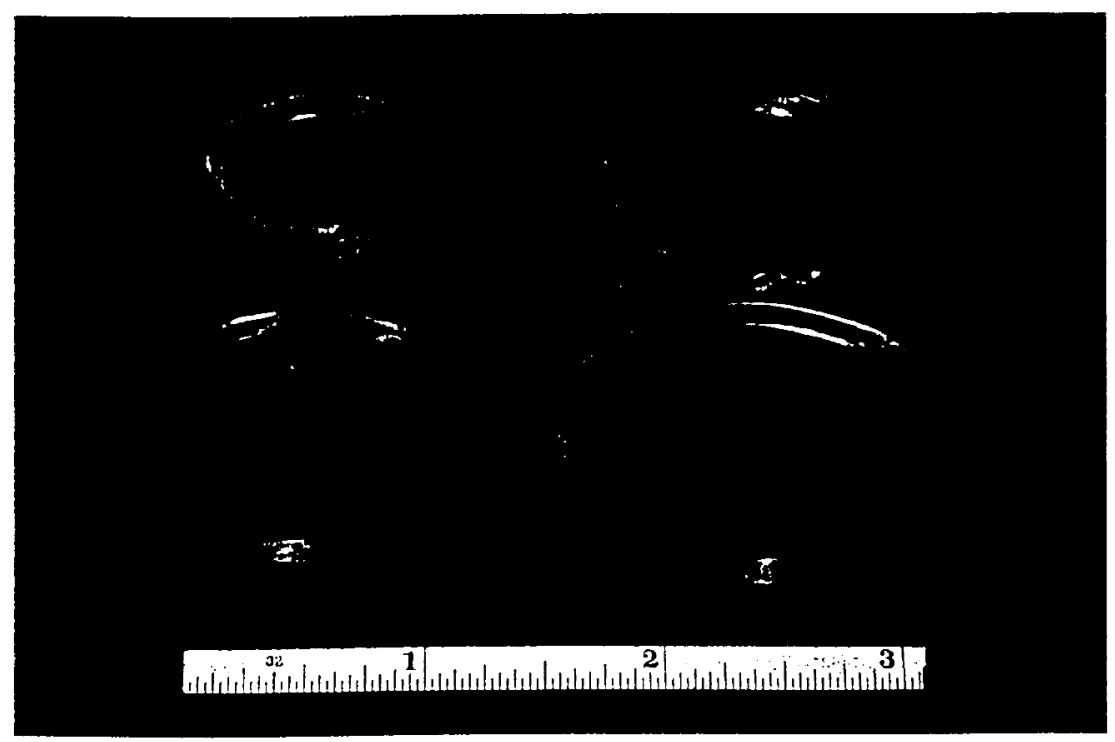

Fig. 38 Picture of crystal slabs cut from a $45^{\circ}$ to $c$-axis grown boule of $\mathrm{Yb}$ :S-FAP from top to bottom (a-d).
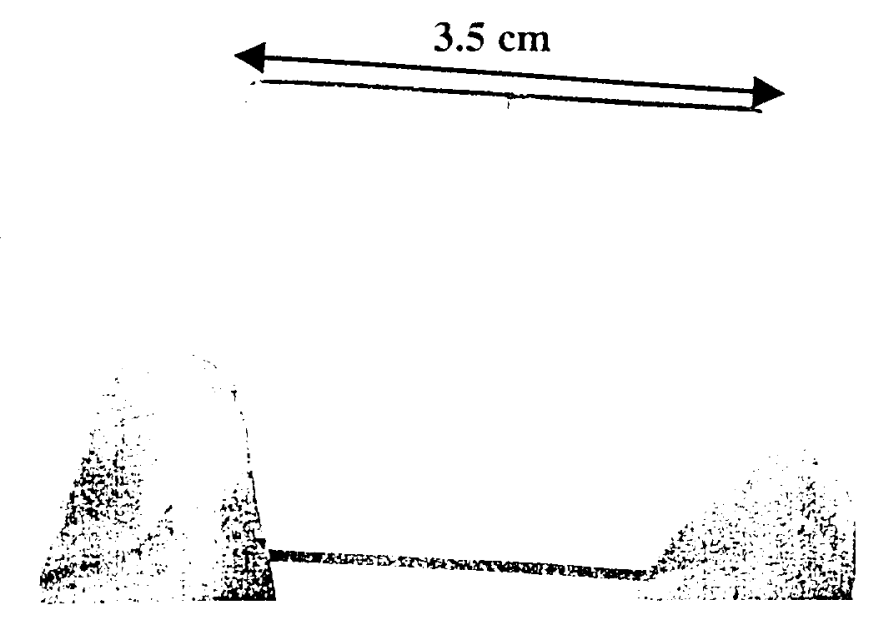

Fig 39. Picture of a 4-piece slab that was recently bonded by ONYX Optics, from slightly sub-scale pieces than will be necessary for $a+x 6 \mathrm{~cm}$ slab for Mercury. 
In summary. significant progress has been made in understanding and controlling each of the defect structures in an effort to develop a reproducible growth process for high optical quality $\mathrm{Yb}$ :S-FAP crystals. To date. over 10 crystals have been grown with the growth profile previously shown in Fig. 37 . Results have produced boules with greatly reduced defects that have optical properties that nearly meet the Mercury specifications. Future crystal growth experiments will be directed at concentrating the bubble core with higher thermal gradients or growing smaller diameter crystals from which subscale slabs can be cut. If high quality crystals of $\sim 4-5 \mathrm{~cm}$ diameter can be grown then two half slabs can be cut and diffusion bonded together. In the case where only smaller diameter crystals can be produced with high optical quality. four $1 / 4$ slabs will be bonded together to yield a full size slab. 


\section{Wavefront Measurements}

Interferometry techniques are utilized as a diagnostic tool for determining the optical quality of the $\mathrm{Yb}$ :S-FAP crystals that are produced. The bulk crystal homogeneity is measured with an interferometer as a means to quantify the crystal quality. Then realistic phase maps for the entire $3 \times 5$ $\mathrm{cm}$ aperture are created from a measured phase map of a subscale crystal for use in the 2-D propagation simulations. The phase maps of the homogeneity are converted to Power Spectral Density (PSD) plots. which are the square of the Fourier amplitude spectrum divided by the width of the frequency intervals (eqn. 3).

$\operatorname{PS} \mid)\left(v_{v}\right)=\int \operatorname{SS} \operatorname{Sin}\left(v_{1}, v_{y}\right) d v_{y}=\int \frac{\left|\operatorname{D}\left(v_{1}, v_{v}\right)\right|^{2}}{\Delta v_{v} \Delta v_{y}} d v_{y}$

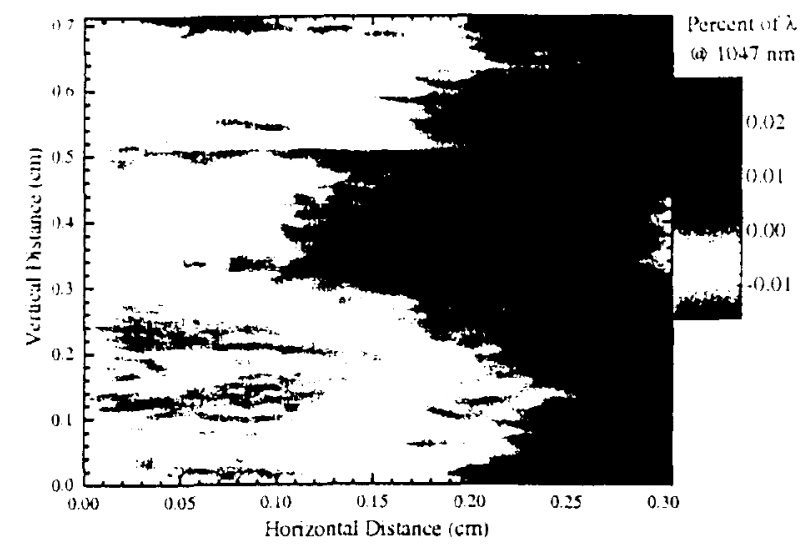

Fig. 41 Phase map of an Yb:S-FAP crystal with a thickness of $6.54 \mathrm{~mm}$.

where PSD $\left(v_{x}\right)$ is the 1-D PSD. PSD $\left(v_{x}, v_{y}\right)$ is the 2-D PSD. $\Phi\left(v_{x}, v_{y}\right)$ is the discrete fourier transform. $\Delta v_{x}$ and $\Delta v_{y}$ are the frequency steps. and $x . y$ can be rotated to any directions of interest. The maximum tolerable PSD in any frequency interval is defined by the desired beam characteristics at the output of the laser system and affects the diffraction limited spot size possible. Propagation calculations have shown that grain boundaries. core defects, or any imperfections visible to the naked eye have an undesirable affect on the transmitted wavefront. Fig. 41 displays the phase map of a section from one of the best $\mathrm{Yb}: \mathrm{S}$-FAP boule grown to date. This phase plot was taken from a clear sub-apertured region of the crystal that still had bubble core defects. The two dimensional PSD plot of the phase map was then integrated along different directions (eqn. 3) to look for discrete frequency components arising from polishing, grain boundaries, scatter defects, etc. As observed in Fig. 42, the sub-apertured region appears independent of the angles considered. Since the measurement is taken over a finite area with a camera having a finite resolution. the regions of validity are restricted to the area between the vertical lines on the plot. The high frequency valid data limit is defined as halt of the maximum frequency that can be displayed by the pixels of the camera where a full wave is five pixels. The low frequency data valid line is defined as six times the lowest frequency. a half wave over the full aperture. In addition. the focus and tilt from the phase data affects the PSD value of the lowest trequencies. The straight line through the data corresponds to a fractal model ${ }^{15}$ given by eqn. + . where $v$ is the spatial frequency. and $\alpha$ and $\beta$ are constants.

$$
F(v)=a v^{-\beta}
$$


The $\beta$ value was determined by actual measurements of high quality optics, while the $\alpha$ value is determined by the desired diffraction limit specification of the laser system. For Mercury, our goal is a $5 x$ diffraction limited within a $3 \times 5 \mathrm{~cm}$ beam.

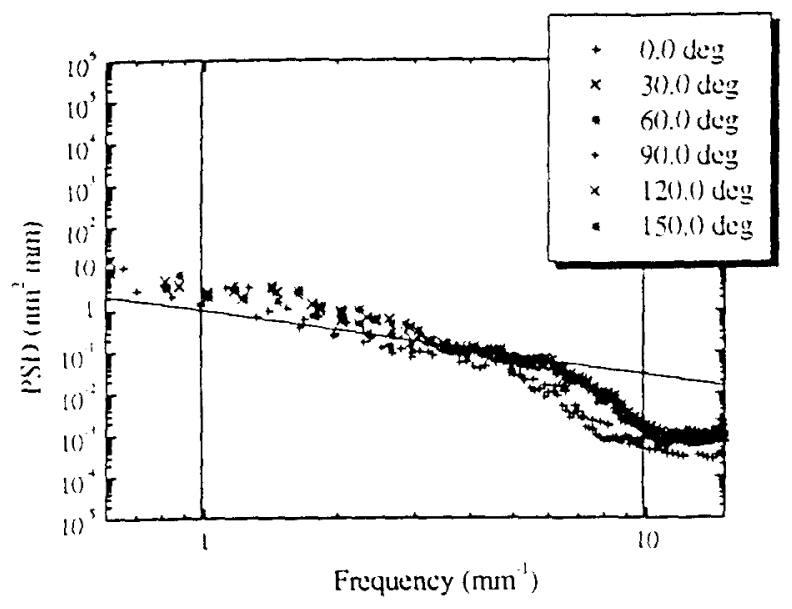

Fig. 42 Power Spectral Density (PSD) plot of Yb:S-FAP crystal $6.54 \mathrm{~mm}$ thick PSD plots for different angular linouts.

\section{Bonding Technique}

In the event that full $3 \times 5 \mathrm{~cm}$ aperture crystals cannot be grown, we have investigated the possibility of diffusion bonding smaller crystals together either in a $1 \times 2$ or $2 \times 2$ bond configuration. Diffusion bonding can offer additional advantages since the core and any other defects in a crystal can be removed by cutting around them and diffusion bonding the defect-free pieces together as shown in Fig. 43. We have been working closely with a company (Onyx Optics, Inc., Dublin, CA) to bond small S-FAP crystals together.

a)

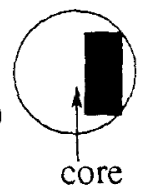

c)

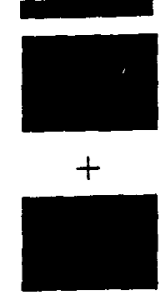

b)

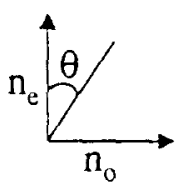

$\longrightarrow$

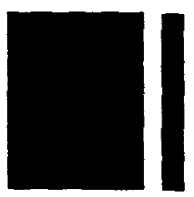

Fig. 43 (a) Boule cross section showing where slabs are cut out of boule with respect to the index/core defects. (b) Coordinate system of the indices of refraction. (c) Cartoon of bonding steps including possible misalignment of crystalline axis. 
The bonded regions of the crystals appear not to have a measurable effect on the phase front. We used an angular bond technique to gradually overlap the two regimes so as not to create a step function effect. The angular orientation that the crystals are cut and bonded is shown in Fig. $+3 b$, where the angle is defined relative to the extraordinary index of refraction. which is along the $c$-axis. This bonding scheme accounts for possible errors in misorientation (Fig. +3a) or differences in crystal homogeneity from slab to slab. Fig +4 shows a phase map of an S-FAP crystal from which a refractive index core section has been removed from the middle and then the two outer pieces were diffusion bonded back together. The vertical dotted lines indicate the width of the bonded region created by the angular overlap. Fig. 45 compares the 1-D PSD of one half of the crystal that does not include the bond to the 1-D PSD of the whole crystal including the bond. Clearly, the two 1-D PSD's show no measurable difference. thus validating this technique as an option for using sub-aperture crystals to produce a full-scale slab. We are also investigating a scheme where + sub-scale $2 \times 3 \mathrm{~cm}$ sections of crystal are bonded together in a two step process to produce the required crystal size as shown in Fig. $43 \mathrm{c}$. Concerns which arise from this more complicated bond include: angular mismatch of the crystalline axis, dopant mismatch for sub-slabs originating from different boules or from top to bottom of a boule. and the strength of a slab with three diffusion bonds.

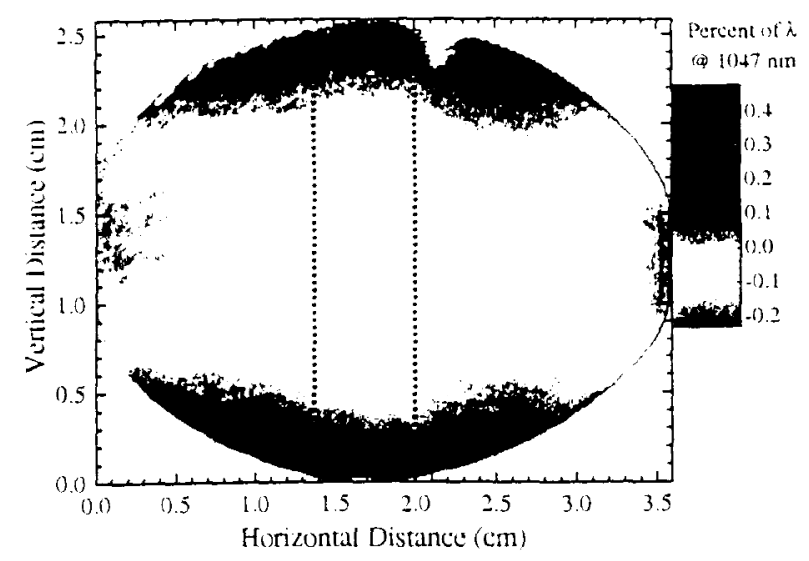

Fig. 44. Phase map of bonded S-FAP crystal

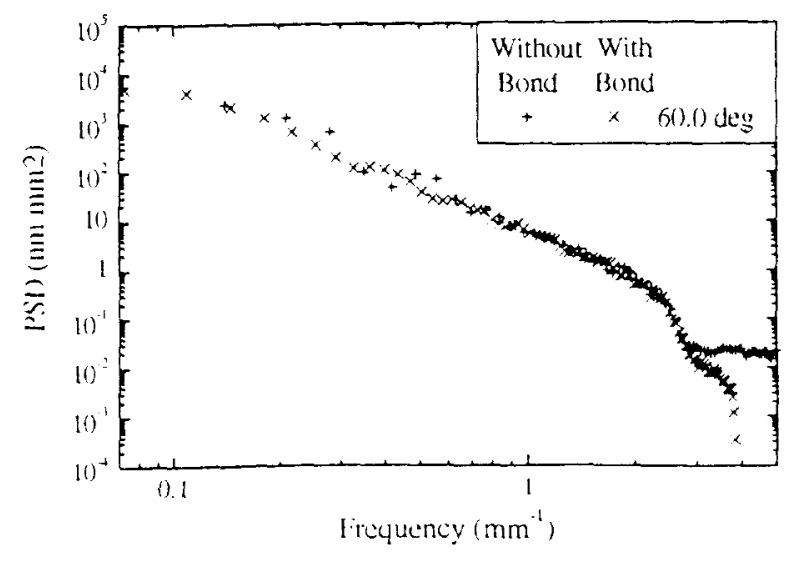

Fig. 45. 1-D PSD comparing the crystal phase map in Fig. 44 before and after bonding. 
As shown in Fig. 46. even for a large angular mismatch of a degree $\left(<0.1^{\circ}\right.$ expected $)$. the optical path difference through the slab is negligible $(\sim 0.01$ wave). X-ray Laue orientation will assure that the angular mismatch of each of the slabs relative to one another is reduced and careful selection of the crystals to be bonded together will be used to address dopant mismatch issues. For example. one boule will yield two half slabs and these slabs will be cut from the same position along the length of the boule and from each side of the core region. Also. a 4 -slab composite was tabricated at Onyx Optics for analysis and to assess the ability to successtully bond four smaller sections of crystal and maintain strength and optical quality. The size of the subscale slabs is affected by the bond angle. $\Omega$. between the slabs, which in turn effects the size of the boules that are required (Fig. +7). The current bond angle is expected to be approximately $15^{\circ}$ to prevent distortion and minimize the diameter of the boules that must be grown. Note that the decision to grow these boules along the a-axis (or c-axis) versus $45^{\circ}$ to the $c$-axis can further reduce the requirement on the boule diameter by as much as $10 \%$.

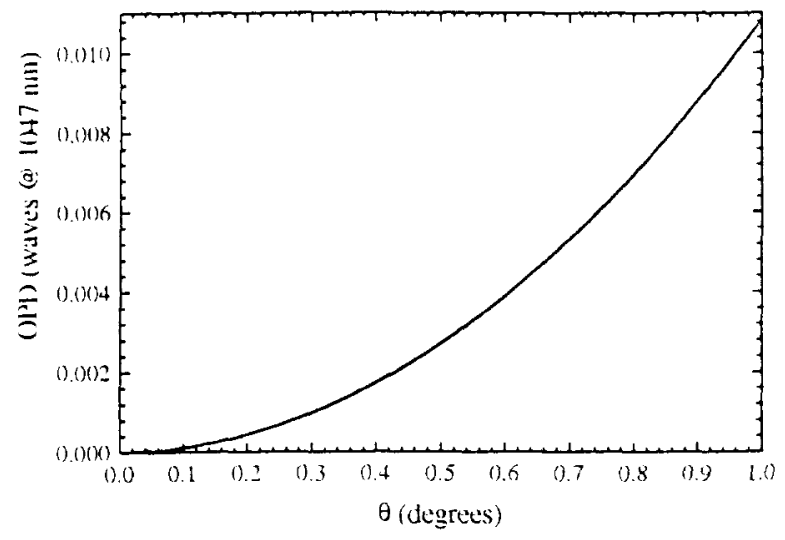

Fig. 46. Optical Path Difference (OPD) given in waves at $1047 \mathrm{~nm}$ (for an S-FAP slab $0.75 \mathrm{~cm}$ thick) vs. angular mismatch of crystalline axis.

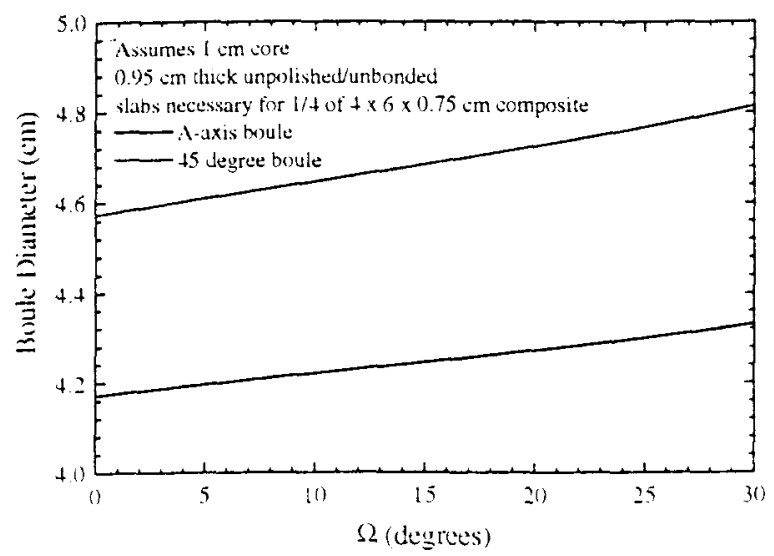

Fig. 47. Required boule diameter for $1 / 4$ slabs with bond angle between slabs. 


\section{Diodes}

\section{A. 900 nm Diode Bars}

The laser diode bars needed for the Mercury laser present several unique challenges. Both the high-peak power and long-pulse operation impose extreme thermal/optical stresses to the output and reliability of the diode bar. In addition. because of the narrow absorption band of Yb:S-FAP crystal, the material growth uniformity as well as the wavelength chirp during the pulse must be kept to a minimum compared to what is usually desired. Finally. it is worth noting that prior to the beginning of this project. no $900 \mathrm{~nm}$ diodes. of any kind. were yet commercially available. So in addition to the performance/reliability requirements that are unique to Mercury. it was imperative to sort out issues specifically related to growing high quality $900 \mathrm{~nm}$ diode material. In particular we were concerned because the $900 \mathrm{~nm}$ wavelength appeared to fall between the AlGaAs and InGaAs semiconductor material systerns. Outlined below are the specific requirements for the diode bars:

- $900 \mathrm{~nm}$ peak wavelength

- $10 \mathrm{~nm}$ FWHM at 750 us pulse (includes chirp and material uniformity)

- 100W peak output power per bar

- $10 \%$ droop over pulse

- 50\% wall plug efficient

- 10:1 polarization (TE)

- $10^{8}$ shot lifetime

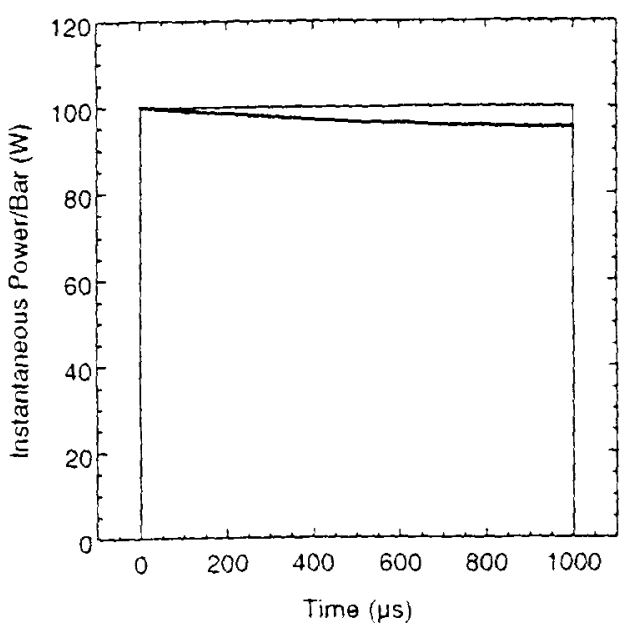

Fig. 48 (a) Instantaneous peak power vs. time into the pulse. Note that the power level (or droop) has decreased by $\sim 5 \%$ at the end of the pulse (i.e.. $750 \mu$ s). (b) Wavelength shift (i.e., chirp) during the pulse is approximately $5 \mathrm{~nm}$.

We have selected CoherentTutcore as a supplier for the $900 \mathrm{~nm}$ diode bars. All of the specifications shown above have been successfully demonstrated. Shown in Fig. 48 is the power droop and wavelength chirp of a typical $900 \mathrm{~nm}$ diode bar at the design point. The droop is kept to within $6 \%$ of the peak power level. which is within our design specifications. Also shown is the wavelength shift during the pulse (often referred to as "chirp"). which is also within specification. Both the droop and wavelength chirp have been modeled previously assuming one-dimensional heat flow with good 
agreement. The excellent thermal performance is due primarily to three factors: diode bar design, cavity length. and heatsink material properties. The diode material used here is "AAA" material: Al-free in the active region. but with $\mathrm{Al}$ contained in the cladding layers. The idea is to remove $\mathrm{Al}$ from the active layer where it would degrade reliability (e.g.. facet damage and bulk defects). while preserving Al-containing compounds in the cladding to maintain good carrier confinement (i.e.. higher band gap) than is possible without Al. The high band-gap cladding layer allows the use of higher band gap confinement layers that mitigate carrier leakage to provide lower threshold current $(18 \mathrm{~A})$. better slope efficiency $(>1 \mathrm{~W} / \mathrm{A})$. and lower droop than is feasible with entirely Al-free structures. Higher efficiency mitigates the effects of positive thermal feedback during the pulse which was earlier observed to be a serious parasitic (in terms of power loss and wavelength broadening). Other improvements include the use of a long cavity ( $\mathrm{I} \mathrm{mm})$ for better heat dissipation, consistent with the excessive pulse widths needed for Yb:S-FAP. In addition. the peak wall plug efficiency is better optimized for $1 \mathrm{~mm}$ cavities, as shown in Fig. 49. Shorter cavities have too high of resistive losses at the design point (100W/bar) while longer cavities ( $>1 \mathrm{~mm}$ ) have excessive parasitic optical loss. Even at power levels as high as $150 \mathrm{~W} / \mathrm{bar}$ the $1 \mathrm{~mm}$ cavity gives nearly optimum performance in wall plug efficiency.

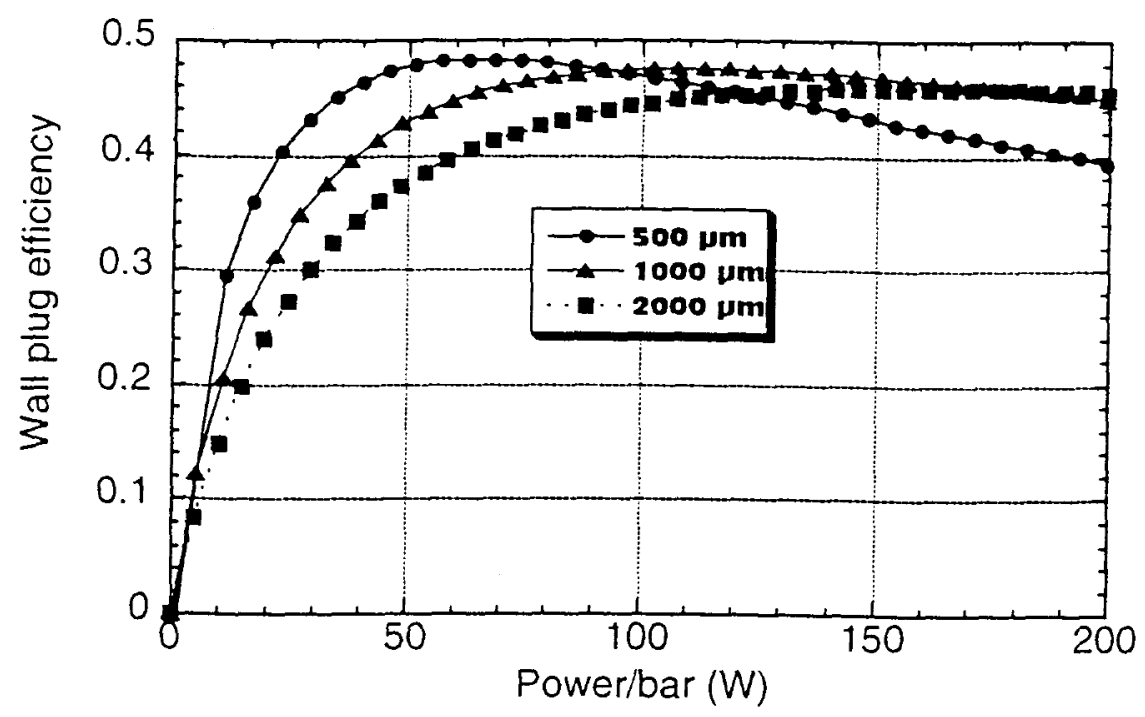

Fig. 49 Wall plug efficiency vs. power level of bar for various cavity lengths. The peak wall plug efficiency is $45 \%$ for the $1 \mathrm{~mm}$ cavity length over a range of $100-150 \mathrm{~W} / \mathrm{bar}$.

Diode lifetime is a complex issue since there are many factors that can negatively impact reliability. Accordingly, many of the key practical considerations will be discussed in later sections. For the moment. we concentrate on early failures and long term degradation mechanisms that are specifically associated with the diode bar itself under normal operation. As stated previously, the AAA material represents an optimum design in performance and reliability due to the incorporation of In and the elimination of $\mathrm{Al}$ in the active layer which reduces early failures and minimizes the propagation of darkline defects. We have not observed early failures. and have shown good long-term degradation rates with our packaging approach. Shown below in Fig. 50 is a typical $900 \mathrm{~nm}$ diode bar operated at $120 \mathrm{~W}$ at the beginning of test under accelerated Mercury conditions ( $750 \mu$ s pulse at $100 \mathrm{~Hz}$ rep. rate). We have tested over a hundred bars that show similar output pertormance and lifetime characteristics. Note that the lifetest is typically operated at $120 \mathrm{~W}$ : the $20 \%$ safety margin is needed to ensure an average $100 \mathrm{~W} / \mathrm{bar}$ over the life of the entire array (i.e.. $640 \mathrm{~kW}$ peak power) planned for Mercury. Because of the anisotropy 
of gain in the Yb:S-FAP crystal. the diodes should be highly polarized. Due to compressive strain in the quantum well. the diode output is polarized in the transverse electric (TE) direction. Measured data on several arrays indicates that the wavelength polarization is $>0.95$ TE polarized.

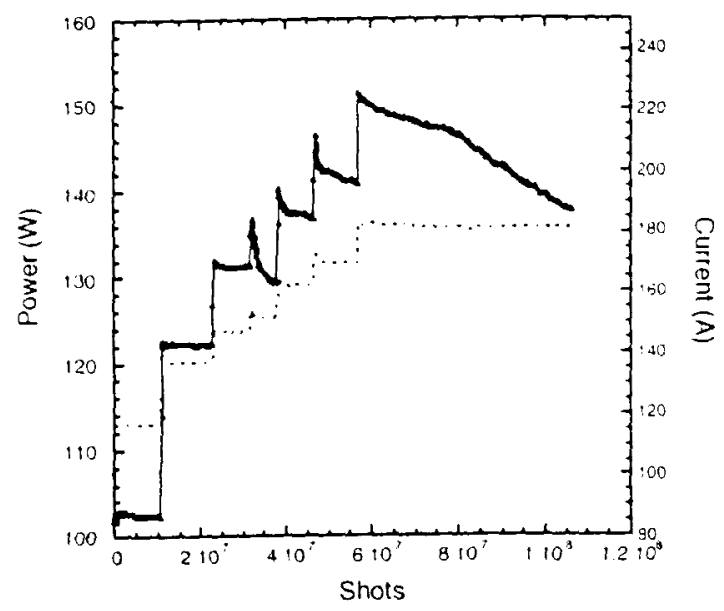

Fig. 50. Laser diode reliability of $900 \mathrm{~nm}$ diode bars. The diode was operated at constant current conditions with the diode junction near room temperature. The $20 \mathrm{~W}$ safety margin at the start of test is ensures reliable power output across the entire diode array.

\section{B. Diode Heatsinks}

Dramatic improvements in both the performance and cost of packaging laser diode arrays will be critical for demonstrating a plausible pathway to advanced target shooters and to IFE. A significant impediment to reducing packaging costs is eliminating the time and effort associated with handling large quantities of discrete components. We have chosen an approach that addresses these issues and introduces the following attributes:

- monolithic (to reduce the number of parts/processes and maintain accurate registration)

- formed from Si substrates (for large volume with micron-precision tolerances)

- reliable (using tluxless thin film Indium soldering process)

- minimal dead space (to increase exitance)

- simplicity (for low cost)

The laser diode array architecture is called Bars And Springs In Slots (V-BASIS) and a schematic is shown in Fig. 51 . The $V$ prefix indicates that this architecture employs a $V$-shape design for mounting the diode bars, and that the laser emission is angled with respect to the substrate. The heatsink submount can be easily fabricated from a Si wafer that is wet etched at a preferred orientation to exploit the highly anisotropic nature of crystal etch planes. A metal layer deposited on the top of the substrate provides electrical continuity of neighboring diode bars, and a notch at the bottom of the $V$-groove produces an electrical break so as to avoid shorting the diode bars. There is a spring (in the shape of a V) that makes contact to the adjacent wall and provides electrical continuity. Since the active layer of the diode bar rests firmly against one side of the groove, a single microlens frame can be used to collimate the emission of all diode bars simultaneously. 
Conceptually, the $V$-groove architecture balances the trade off between two competing problems for "IFE-like" diode arrays. On the one hand. a high peak irradiance is desirable which tends to reduce the diode spacing. However. the diode spacing must be kept to some minimum spacing to prevent thermal cross talk between neighboring diodes during the thermal transient of the pulse. In the case of long pulses $(0.75-1 \mathrm{~ms})$ appropriate for IFE. the diode spacing must be kept greater than $\sim 500 \mu \mathrm{m}$. With the $\mathrm{V}$ groove design, there is negligible thermal cross talk while still maintaining a reasonably close packing density. The full angle of $109.5^{\circ}$ produces a $1.7 \mathrm{~mm}$ linear pitch of the diode (which increases the thermal footprint). but allows an optical pitch of $1.0 \mathrm{~mm}$ to increase the effective exitance. Note that the $\mathrm{V}$-groove also allows other manufacturing advantages (such as a simple means for diode insertion and inspection of the submount) that lead to a reliable. low-cost package.

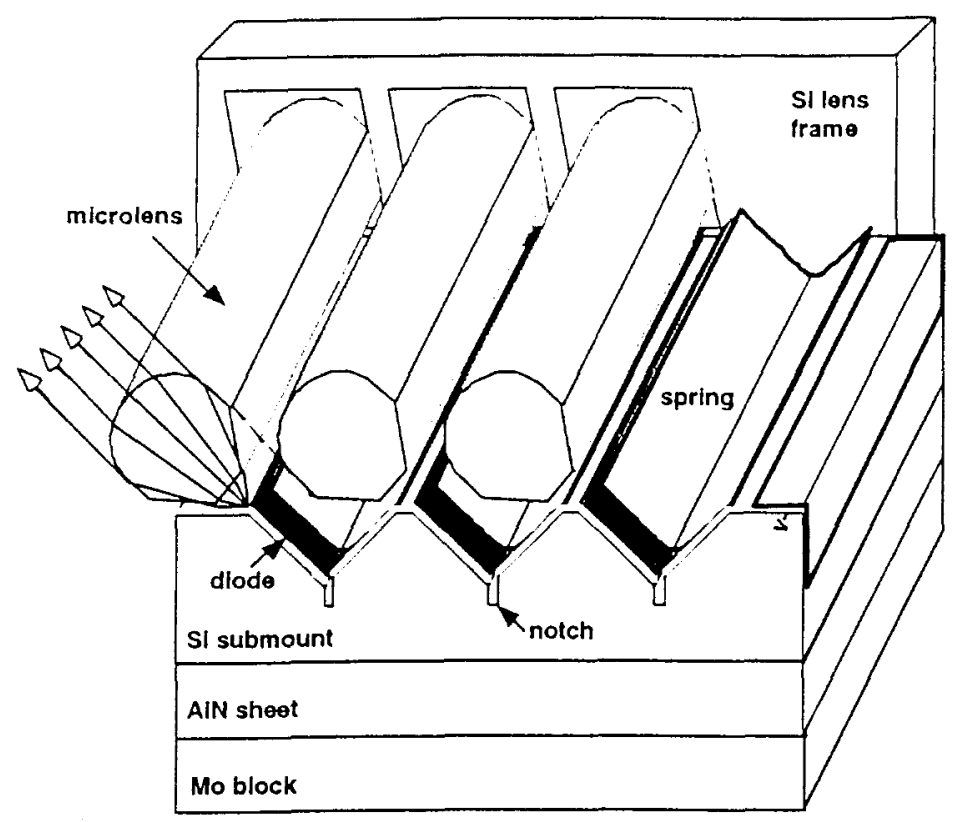

Fig. 51. Schematic diagram of V-BASIS laser diode array ( 3 of 23 diode bars are shown). Both the lens and laser diode array have precise registration to enable a single active lens alignment.

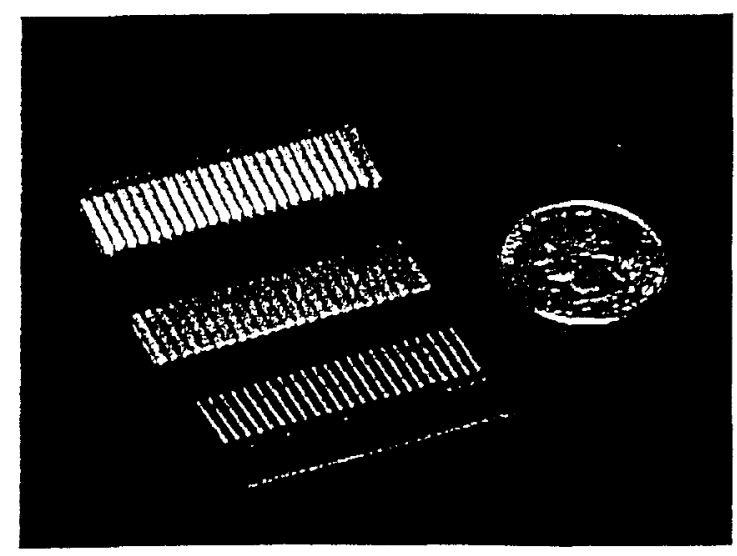

Fig. 52. Schematic diagram of V-BASIS "tile". The tiles are bolted to a larger cooled backplane in a modular fashion. 
A prototype V-BASIS tile is shown in Fig. 52. Each "tile" is $1 \mathrm{~cm} x+\mathrm{cm}$. which can hold 23 diode bars in series. We have built and characterized earlier prototypes on a related heatsink called BASIS. which used BeO and required precision machining. Diode bars have been tested up to 1 t0 A with no roll over and a slope efficiency of $\sim 1 \mathrm{~W} / \mathrm{A} / \mathrm{b}$ ar. The peak wall plug efficiency is $+5 \%$ at the design point (note that the actual laser diode efficiency is slightly higher than shown since the background resistance has not been subtracted from the measurement). The $900 \mathrm{~nm}$ diode bars have been lifetested on this prototype packige under accelerated conditions (as high as $140 \mathrm{~W}$ peak power at $100 \mathrm{~Hz}$ and 750 us with no failures. The long-term degradation is similar to that shown previously in Fig. 50 .

Previously, we had investigated the proper heatsink material to be chosen for Mercury-operated diodes. In this regine. the thermal stresses are greatest during the transient pulse. so that a figure of merit (FOM) could be associated with a material in its ability to prevent heating of the laser diode during that pulse. The $\mathrm{FOM}$ is given by $[\mathrm{pCK}]^{1 / 2}$ (i.e.. the temperature rise is inversely proportional to the square root of the volumetric heat capacity times the thermal conductivity). Note that this expression assumes one-dimensional semi-infinite heat flow for time limits appropriate to Mercury. There are several key trade offs determining an optimum choice of starting material. including: thermal performance. cost. manufacturability. electrical conductivity, toxicity, and thermal expansion mismatch. The two most promising candidate materials were determined to be $\mathrm{BeO}$ and Si. From a thermal-performance point of view, $\mathrm{BeO}$ was preferable in having a $\sim 40 \%$ higher FOM than Si (Fig.53).

However. it was unknown if the heatsinks could be fabricated within the requisite tolerances and cost goals suitable for Mercury. Accordingly, we began a parallel $R \& D$ effort to investigate precision sawing/fabrication of BeO heatsinks. After exhausting the most advanced manufacturers (both external and internal to LLNL), we concluded that state-of-the-art machining had not advanced to the point to be acceptable as feature control and tolerances were insufficient for producing high-radiance, low cost. high volume. highly reliable diode arrays based on BeO. The Si-heatsink was thereby down selected as the baseline Mercury approach. It is noteworthy that the remarkable improvements in diode bar performance that Coherent achieved led to a near equivalence of $\mathrm{BeO}$ and Silicon heatsink performance. Therefore, the final outcome of identifying and developing a low cost. high performance diode array technology was successfully attained.

In order to mitigate the effects of wavelength chirp, we designed the V-BASIS architecture to accept $1 \mathrm{~mm}$ cavity length diodes (which decreases the thermal heat flux at the diode junction). Shown in Fig. 53 is the wavelength chirp as tunction of time into the pulse under Mercury conditions (i.e.. 100 W/bar). This corresponds to a thermal flux of $\sim 1.2 \mathrm{~kW} / \mathrm{cm}^{2}$ at the junction. At the design point of 750 us. this produces a wavelength chirp of $5 \mathrm{~nm}$. When the wavelength chirp is convolved with the intrinsic spectral bandwidth of the array, this only contributes $\sim 0.5 \mathrm{~nm}$ of additional spectral broadening, which is acceptable to the system.

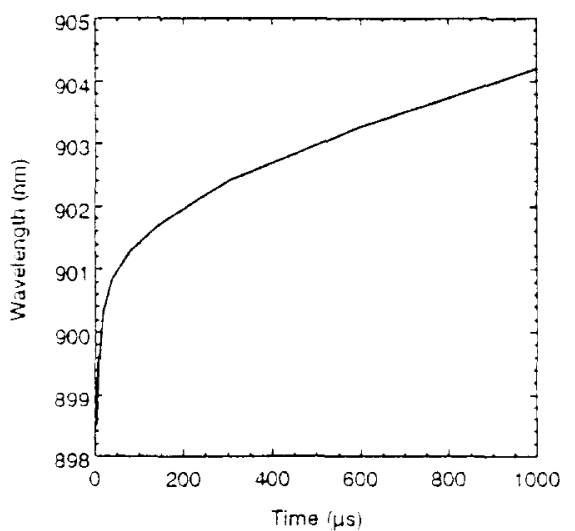

Fig. 53 Wavelength chirp as a function of time into the pulse under Mercury conditions. The wavelength chirps $-5 \mathrm{~nm}$ for a 750 us-long pulse in agreement with thermal calculations. 


\section{Microlensing}

High brightness is a critical feature of the Mercury diode array. Outlined below are the specific requirements:

- 40 mrad x 200 mrad collimated angular divergence (at $85 \%$ of the CDF or cumulative distribution function)

- minimal dead space

- manufacturable (formed from Si substrates with micron-precision tolerances)

- robust

- low cost (single active alignment)

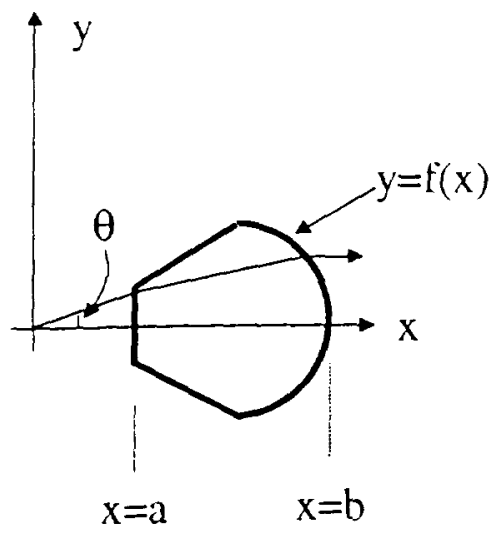

Fig. 54 The coordinate system used to design the plano-convex cylindrical-microlens used in with the vBASIS diode array. The diode aperture is located at the origin and $\theta$ denotes the launch angle of various rays with respect to the optic axis.

A sketch of the cross-sectional protile of the plano-convex shaped-cylindrical-microlens that is used to collect and collimate the output radiation of the laser diode bars in the v-basis arrays is shown in Fig 54 . The profile on the curved output surface of the lens is designed using a variational calculation based on Fermat's principle. The result of this calculation is a set of coupled first-order differential-equations that describe the curved output surface of the lens in terms of the Cartesian coordinate pairs $(x, y)$,

$$
\begin{aligned}
& {\left[\begin{array}{cc}
(x-a)-\frac{1}{n} \sqrt{(x-a)^{2}+(y-a \tan \theta)^{2}} & y-a \tan \theta \\
\frac{-(y-a \tan \theta)}{(x-a)^{2}} & \frac{1}{(x-a)}
\end{array}\right]\left[\begin{array}{l}
\frac{d x}{d \theta} \\
\frac{d y}{d \theta}
\end{array}\right]=} \\
& {\left[\begin{array}{c}
-\frac{1}{n} \frac{a-\sin \theta}{\cos ^{2} \theta} \sqrt{(x-a)^{2}+(y-a \tan \theta)^{2}}+(y-a \tan \theta) \frac{a}{\cos ^{2} \theta} \\
\frac{a}{(x-a) \cos ^{2} \theta}+\frac{n^{2} \cos \theta}{\left(n^{2}-\sin ^{2} \theta\right)^{3 / 2}}
\end{array}\right]}
\end{aligned}
$$

where $\mathrm{n}$ is the refractive index of the lens material and $\theta$ is a parametric variable that denotes the launch angle of various rays with respect to the optic axis. The solution to these coupled equations is implemented numerically, and uses the initial condition $x(\theta=0)=b$ and $y(\theta=0)=0$. 
The microlenses shown above were fiber-pulled from a precision-ground boule. To minimize/optimize angular divergence. the lens diameter is only sligthly smaller ( $4 \%$ ) than the optical pitch of the diode array. Precision lens frames were fabricated from Si via wet etching. V-grooves formed in the frame can maintain tolerances within $\pm 2 \mathrm{um}$. Note that the key advantage of this approach is a monolithic architecture for reducing cost while preserving. and perhaps improving the angular divergence. One of the most time consuming steps is the active alignment step. and in this case the process is accelerated by over an order of magnitude compared to a single lense-bar alignment procedure. We have verified that the angular divergence is within our expectations. Fig 55 shows a plot of the angular divergence (fast axis) for the same array after microlensing. The collimated angular divergence is $25 \pm 5 \mathrm{mrad}$ with $85 \%$ collected emission.

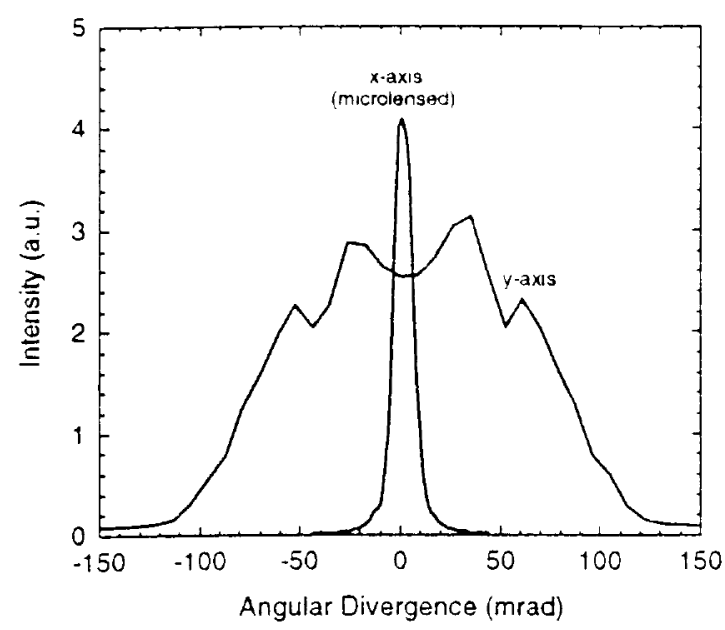

Fig. 55 Angular divergence of a monolithic lens frame assembly. The fast axis divergence is collimated to $25 \pm 5 \mathrm{mrad}$ with $85 \%$ of the collected light.

Note that to increase brightness and power levels the diode bars are designed with a high fill factor (i.e., $90 \%$ of the active region lases). Given that the aperture is virtually "full", the etendue limit prevents us from gaining any significant advantage by collimating the slow-axis direction (i.e., parallel to the diode active layer).

\section{Backplane Cooler}

Integration of the diode array tiles into a full array assembly is a challenging task with various trade offs. We designed the backplane with the following goals in mind:

- minimal dead space (for high exitance)

- negligible array pointing errors $(<1 \mathrm{mrad})$

- minimize optical delivery losses

- adequate thermal performance

- negligible thermal gradient (to avoid wavelength broadening)

- maintainability of the array (modular tiles)

- mechanical/environmental protection of the diode arrays

As shown in Fig. 56, the concept chosen was to use a very large fluid-cooled backplane that individual diode array "tiles" are mounted. This scheme is modular so that they can be individually removed in case of failure. To enhance brightness. the dead space between tiles is minimized to $\sim 25 \%$ (to allow for lens 
frames, electrodes. and mechanical tolerances). Electrical power is brought up through the backside of the backplane. The positive electrode attaches to one side of the tile. and all tiles share a common ground (i.e.. the backplane itselt). The bottom of each tile is tapped to accept bolts that come though the backplane for good alignment (i.e.. horizontal, vertical and angular). In addicion. there is an indium foil gasket (not shown) between the mating surfaces to ensure good thermal contact. Adjacent to the bolts are coolant channels that are $20 \mathrm{~mm} \times 0.5 \mathrm{~mm}$ in size. The deep. narrow channels are necessary for reducing the thermal impedance of the system. $R_{t h} \sim 1.2{ }^{2} \mathrm{C}-\mathrm{cm}^{2} / \mathrm{W}$. Despite the relatively low average power under Mercury conditions. the thermal power dissipated is a serious issue for the performance/reliability of the diodes. as well as for preserving wavelength control. At the design point. the junction temperature of the diodes is specified to have a temperature rise of $-15^{\circ} \mathrm{C}$ (roughly same amount of temperature rise during a single 750 us pulse).

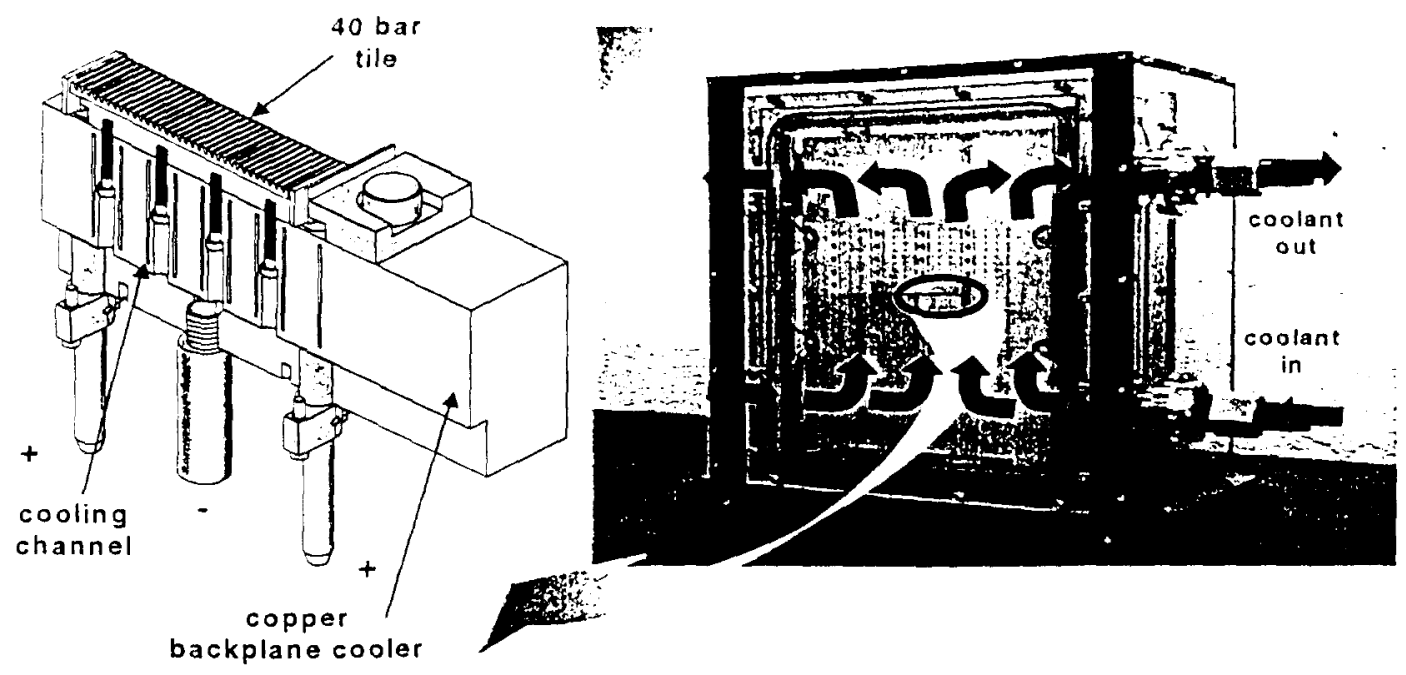

Fig. 56 The backplane assembly. The tiles are modular and can be replaced individually. The electrical feedthroughs and coolant channels are located though/on the backplane itself to minimize dead space across the array.

The backplane has been fabricated and experimentally verified using prototype diode array tiles. Ten 900 nm microlensed BASIS tiles were mounted onto the backplane and tested under Mercury conditions: 100 W/bar, $750 \mu \mathrm{s}$, at $10 \mathrm{~Hz}$ rep. rate. In order to test for thermal cross-talk effects, the wavelength spectra were taken for all ten tiles separately. When individual spectra for each tile are convolved together, the data matches the overall spectrum indicating that there is negligible steady-state thermal cross-talk between neighboring tiles. High flow rates are used to prevent any significant caloric rise that would lead to thermal wavelength broadening across a row of adjacent tiles $\left(\Delta \mathrm{T}<1^{\circ} \mathrm{C}, \Delta \lambda<0.3 \mathrm{~nm}\right.$ ). The backplane is designed to operate from $-40^{\circ} \mathrm{C}$ to $20^{\circ} \mathrm{C}$. This wide temperature range allows for more flexibility of repetition rate and wavelength tuning. To prevent any possible condensation. the diodes are hermetically sealed and are insulated by an outer enclosure that provides adequate thermal insulation. as well as mechanical protection of the diodes. The diode array backplane was characterized in Figs. 57-59. As shown in Fig. 57. the wavelength can be tuned over a very wide range $\left(\mathrm{d} \lambda / \mathrm{dT}=0.33 \mathrm{~nm} /{ }^{\circ} \mathrm{C}\right.$ for $900 \mathrm{~nm}$ diodes). As expected. there is virtually no bandwidth variation observed over the tuning range of interest. We have also characterized the diode array performance as a function of repetition rate (Fig. 58) and duration to address long-term wavelength stability (Fig. 59). There is little wavelength broadening due to the increase in repetition rate. and the wavelength of the diode-array ensemble is stable for any length of time. From these data it appears that all of the backplane performance specifications were comfortably satistied. 


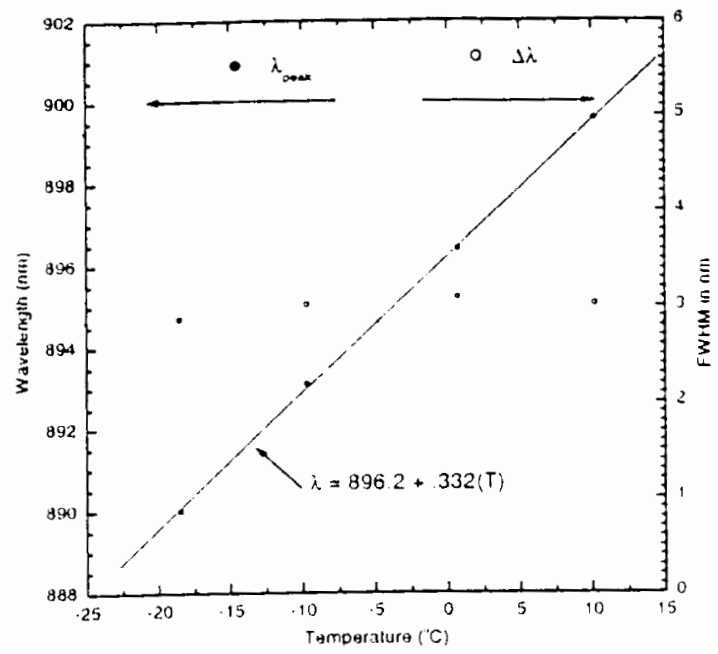

Fig. 57 Peak wavelength and spectral bandwith versus temperature. The wide tuning range enables efficient wing pumping of surrogate $\mathrm{Nd}$ :glass slabs.

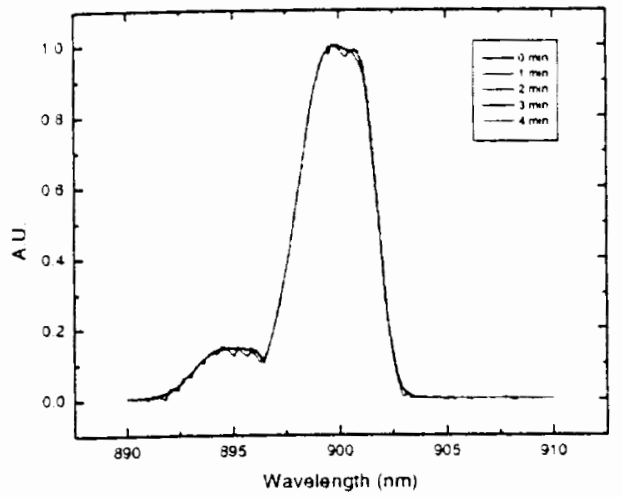

Fig. 58 Wavelength spectrum as a function of time. Thermal equilibrium is reached in under a minute and remains stable for long periods

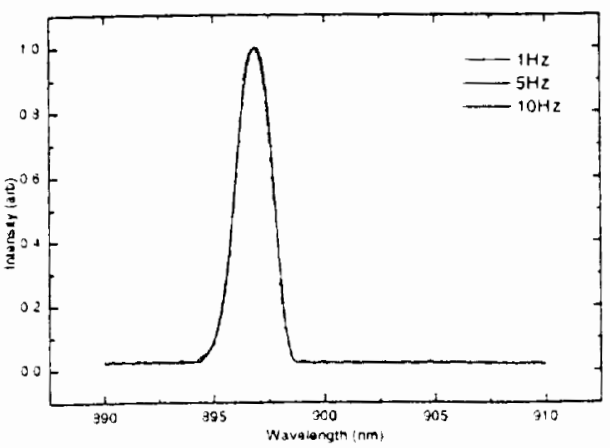

Fig. 59 Wavelength spectrum as a function of repetition rate. The increase in repetition rate has no change on the peak intensity. and shifts the peak wavelength $<0.5 \mathrm{~nm}$. 


\section{E. Future Directions}

As presently visualized, the largest single expense in the construction of a laser-driven IFE power plant will be the laser diode pump arrays. A 1000 -megawatt plant will require approximately 20 gigawatt of peak diode pump power. In order for such a plant to be profitable. it has been estimated that the price of the pump arrays must be less than $7 \mathrm{e}$ per peak Watt ${ }^{32}$. This would make the cost of the arrays $\$ 1 . t$ billion - still a substantial sum but much less than the $\$ 200$ billion that would be required at today"s market price of $\$ 10$ per peak Watt.

Reducing diode costs by more than two orders of magnitude over the next $t 0$ years is an aggressive but achievable goal. Given the incredible cost reductions seen over the past twenty years in the silicon integrated circuit (IC) industry it would seem reasonable that high-power laser diode costs might follow a similar model. and yet diode costs remain stubbornly high. This points out the major economic difference between the costs of laser diodes and silicon ICs. The IC market is a highly competitive. highvolume commodity market with small protit margins (generally a few tens of percent). while the highpower laser diode industry remains a low-volume "specialty" market dominated by a few companies. with much larger profit margins $(300 \%$ to $600 \%$ ). In addition. the technology for manufacturing ICs has been developed to a high art with extremely high levels of process automation while laser diode fabrication remains a manual labor-intensive process. In order to realize the cost goals, the laser diode industry must follow the path of the silicon IC industry.

It is very illuminating to examine today's costs of producing high-power laser diode bars. Consider a $1 \mathrm{~cm} \times 1 \mathrm{~mm}$ diode bar capable of producing $100 \mathrm{~W}$ peak at $900 \mathrm{~nm}$. The retail price for this bar is approximately $\$ 200$, making the cost of unmounted diode bars $\$ 2$ per peak Watt. Mounting this bar in an appropriate heatsink adds another $\sim \$ 5$ per Watt. bringing the total cost of ready-to-use diode power to around $\$ 7$ per peak Watt which is consistent with the best price quotes today from commercial suppliers. Let us now compare these prices to the actual estimated cost of production. The total cost of materials for the diode bar is approximately $\$ 7(\$ 0.07 / \mathrm{W})$, and the labor required to produce it is about 0.4 hours. which costs $\$ 20(\$ 0.20 / \mathrm{W})$ assuming a loaded labor rate of $\$ 50 /$ hr for assembly personnel. Thus, the time-and-materials required to produce a single bar is $\$ 27$. Facility overhead and depreciation expenses roughly double this. making the total cost of production $\sim \$ 50$. Recalling that a typical bar sells for approximately $\$ 200$, the price mark-up is seen to be $400 \%$. Such a large profit margin is indicative of both the small market and lack of competition. The cost of packaging bars into arrays depends on the particular approach taken, but analyses of several different approaches has shown that 50 to $75 \%$ of the loaded production cost of a finished diode array is attributable to packaging expenses.

What is seen from the analysis above is that the present price drivers are manual labor, facility and depreciation expenses, and most importantly a modest market size. Manual labor expenses can be reduced through process automation. Present diode bar manufacturing processes are mostly lacking in automation. in part because of the expense of developing extremely specialized robotic equipment for such a small market. Facility and depreciation contributions to cost are minimized by maximizing the utilization of the facility - all equipment in the manufacturing line should be in constant use for three shitts of operation. This is quite different from typical diode bar fabrication operations where most equipment sits idle much of the time. However. operating a diode fabrication at full capacity only makes economic sense if there is sufficient market to absorb products that are manufactured: this is not presently the case for laser bars. Profit margins will remain high until there is a much larger market with healthy competition. Today"s small diode bar market presents a classic "chicken-or-egg" situation: until the market is larger. manufacturers will not shift to high-volume and low-cost fabrication techniques. but today's high prices are impeding market growth.

In a "fusion economy" the diode bar market situation changes drastically. Instead of today"s market size of several tens of thousands of bars per year. the market would be on the order of hundreds of million bars per year. This enormous shift would propel the diode bar industry towards the silicon IC industry model. We have done a detailed projection of the future cost of peak diode power for a 
manufacturing facility producing 10 gigawatts of ready-to-use peak diode power each year. We make the following assumptions:

- $200 \mathrm{~W}$ peak per diode bar (increased from today's $100 \mathrm{~W}$ )

- Large-volume discounts for raw materials

- 3 shitt plant operation

- 5 year facility depreciation

- Fully-automated tibrication. assembly and testing

- $80 \%$ overall process yield

- Sale price is twice the fully-loaded cost of production (as in the silicon IC industry. $\sim 10 \%$ is profit and the remainder is R\&D. advertising. etc.)

The most challenging assumption in this projection is that of full automation. Laser bars are extremely fragile components - at the later stages of their manufacture they are only $100 \mu \mathrm{m}$ thick. as thick as a human hair - and their reliability is very sensitive to how they are handled throughout the manufacturing process. Although the fabrication of silicon ICs is far more complex. for various reasons their tolerance of robotic handling is much greater than that of diode bars. Nonetheless. we are confident that appropriate specialized robotic equipment can be developed for diode bar manufacture: the difficulty lies in estimating its cost and capacity. We have attempted to make conservative but reasonable projections based on today's technology so as not to underestimate the total cost of diodes produced.

Our projections for the $10 \mathrm{GW} / \mathrm{yr}$ diode bar array factory result in a facility reminiscent of today's silicon IC fabrication. It has 130 employees devoted to the fabrication of diode bars and another 580 devoted to bar mounting and array assembly and test. The facility and its 125 pieces of major fabrication and test equipment have an initial value of $\$ 390 \mathrm{M}$. The yearly cost of operation (wages, supplies, depreciation, utilities and maintenance) is $\$ 211 \mathrm{M}$. We find that this factory can produce ready-to-use diode power at a sale price of $5.6 \%$ per peak Watt. This projection is encouraging in that it is less than the $7 \notin / W$ calculated by Orth $^{32}$ as the maximum cost for profitable power plant operation. We emphasize that that the uncertainties in our model are quite large. We will refine our model and projections as the technologies advance. 


\section{Diode Power Conditioning}

The basic element in the diode power conditioning system is the LDD-200 pulse current source manufactured by Directed Energy. Inc. of Fort Collins. Colorado where aspects of the module were designed in conjunction with LLNL electrical engineers. Each pulser is connected to a single laser diode tile through a low inductance strip line cable (Fig. 57a). The sources are mounted in overhead racks above the laser tables. External DC power is shared among several pulser chassis. the number pulsers sharing a common supply will vary depending on the required output power. The driver consists of two independent channels in a common enclosure. The drivers share common AC support power. DC high voltage and water-cooling. Each channel is independently controlled via an RS-485 port. The driver provides analog. real-time voltage and current monitors that can be monitored using an oscilloscope or digitizer for real-time data acquisition of the voltage and current profiles for each pulse during operation. In addition. a digitized sample of the current and voltage waveform amplitudes are available through the RS-485. The electrical specifications and limitations are listed in Tables 3 and 4.

The driver features advanced circuitry to protect both the diode and driver. These features include: A relay closure that mechanically shorts the output to ground when power to the driver is turned off. The internal capacitor bank is also shorted to ground. At turn on, at any time the driver is not pulsing. and during a fault condition, the output is electronically crow-barred to ground, ensuring that no current flows through the diode except during the on period of the pulse. If a fault is set during operation. the output pulse is truncated (turned off) prior to activation of the crow-bar circuitry.
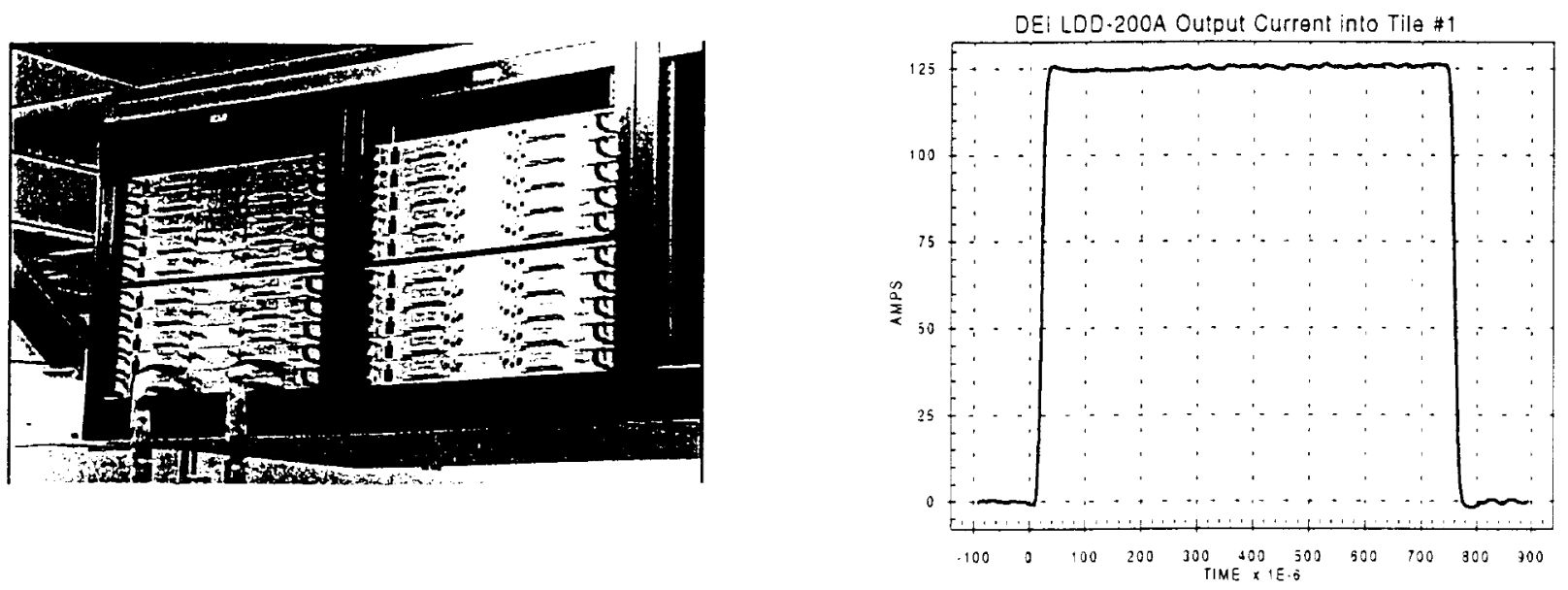

Fig 57 (a) Photo of 20 rack-mounted 2-channel pulsers installed in Mercury. (b) Typical output current waveform.

The nominal operating point for a single laser diode tile is $70 \mathrm{~A}$ at $80 \mathrm{~V}$ for $750 \mathrm{uS}$ at $10 \mathrm{~Hz}$ (example waveform Fig. 57b). Each channel of an LDD-200 can deliver up to $200 \mathrm{~A}$ at $150 \mathrm{~V}$ for $1.5 \mathrm{~ms}$ with a $20 \mathrm{~Hz}$ repetition rate. This allows for a very flexible experimental envelope and leaves plenty of capacity for future expansion. The current sources monitor themselves for internal failures and "out of specification" computer commands. If a fault is detected the output pulse is immediately terminated. a "crowbar" switch is placed across the output. the high voltage DC power supply is disconnected. and the internal capacitor bank is discharged. A fault indicator lights on the front panel and fault code is made available to the control system. The pulser may not be re-enabled until the fault condition has been cleared. 
Table 3. Electrical Specifications

\begin{tabular}{|c|c|}
\hline PARAMETER & VALUE \\
\hline \multicolumn{2}{|l|}{ PULSE OUTPUT CURRENT } \\
\hline Amplitude Range & $5 \mathrm{~A}$ to $200 \mathrm{~A}$ in $1 \mathrm{~A}$ steps \\
\hline Pulse Rise and Fall Time & $\leq 50 \mu \mathrm{s}(10 \%-90 \%)$ \\
\hline Pulse Width & $100 \mathrm{us}$ to $2 \mathrm{~ms}$ in $500 \mathrm{~ns}$ steps \\
\hline Pulse Recurrence Frequency Range & Single Shot to $20 \mathrm{~Hz}$ \\
\hline Maximum Duty Cycle and Average Current & $5 \%$ \\
\hline Pulse droop and ripple (@200) $2 \mathrm{~ms} \mathrm{PW}$ ) & $<1 \%$ \\
\hline Voltage Over/undershoot & $<2 \%$ \\
\hline Output Connector & Sub- $\Omega$ Micro-Stripline. Front Panel \\
\hline \multicolumn{2}{|l|}{ COMPLIANCE VOLTAGE } \\
\hline Range & $15 \mathrm{~V}$ to $150 \mathrm{~V}$ in $1 \mathrm{~V}$ steps \\
\hline \multicolumn{2}{|l|}{ CURRENT LIMIT } \\
\hline Range & $5 \mathrm{~A}$ to $200 \mathrm{~A}$ in $1 \mathrm{~A}$ steps \\
\hline \multicolumn{2}{|l|}{ VOLTAGE LIMIT } \\
\hline Range & $15 \mathrm{~V}$ to $150 \mathrm{~V}$ in $1 \mathrm{~V}$ steps \\
\hline \multicolumn{2}{|l|}{ TRIGGER IN } \\
\hline Type & Positive Edge Trigger \\
\hline Trigger Input & $\begin{array}{l}\text { TTL or }+5 \mathrm{~V} \pm 1 \mathrm{~V} \text {. into } 50 \Omega \text {, 100ns minimum } \\
\text { width }\end{array}$ \\
\hline \multicolumn{2}{|l|}{ CURRENT MONITOR OUTPUT } \\
\hline Analog CVR Monitor & $50 \mathrm{~A} / \mathrm{V}$ into $50 \Omega . \pm 1 \%$ \\
\hline Digital Current Monitor & 12 Bit AVD with pulse profiling capability \\
\hline \multicolumn{2}{|l|}{ VOLTAGE MONITOR OUTPUT } \\
\hline Analog Voltage Monitor & $50 \mathrm{~V} / 1 \mathrm{~V}$ into $50 \Omega . \pm 1 \%$ \\
\hline Digital Voltage Monitor & 12 Bit A/D with pulse profiling capability \\
\hline \multicolumn{2}{|l|}{ GENERAL } \\
\hline Dimensions ( $\mathrm{H} \mathrm{XWX} \mathrm{X})$ & $13 / 4 " \times 17^{\prime \prime} \times 21^{\prime \prime}$ \\
\hline Cooling & Water@1GPM.40 0 max. inlet temperature \\
\hline
\end{tabular}

Table 4. Comparison of diode operating points and pulser limitations

\begin{tabular}{|l|l|l|}
\hline PARAMETER & $\begin{array}{l}\text { MAXIMUM LASER DIODE } \\
\text { OPERATING POINT }\end{array}$ & $\begin{array}{l}\text { MAXIMUM PULSER } \\
\text { OUTPUT }\end{array}$ \\
\hline Current & $130 \mathrm{~A}$ & $200 \mathrm{~A}$ \\
\hline Voltage & $90 \mathrm{~V}$ & $150 \mathrm{~V}$ \\
\hline Pulse Width & $750 \mathrm{uS}$ & $2 \mathrm{mS}$ \\
\hline Repetition Rate & $10 \mathrm{~Hz}$ & $20 \mathrm{~Hz}$ (at output above) \\
\hline Ave. Power Output & $88 \mathrm{~W}$ & $1200 \mathrm{~W}$ \\
\hline
\end{tabular}




\section{Laser Diode Protection System}

The laser diodes represent a significant cost factor in the Mercury Laser. Protection of these diodes during operation is a major design consideration of the pulsed power system. The pulsers developed for the Mercury Laser have a large reserve of available power and in fact are capable of delivering at least a factor of $1+$ more power than required at the maximum operating point of the laser diode tiles.

In normal operation it would be quite possible for the pulser to destroy its diode load. Additionally at the nominal operating point each pulser's capacitor bank stores approximately $200 \mathrm{~J}$. In the event of a pulser failure this energy must not be delivered to the diode.

A four-pronged approach is taken to the problem:

1. Intelligent operators

2. Control Software to keep the diodes in their safe operating region regardless of operator input

3. Firmware within the pulser units

4. Hardware limits for some parameters and pulser faults

Each critical parameter and its solution is described below with a summary outlined in Table 5.

\section{Diode Current}

The control system software prevents the operator from requesting current outside the safe operating region. This value is stored in a computer database that can only be edited by an administrative user with the proper password.

The pulsers will reject any command outside of it's operating parameters. The pulser will continue to operate as before the illegal command, but an error status will be returned to the computer control system. Currently this limit is set at $220 \mathrm{~A}$ ( $10 \%$ above the $200 \mathrm{~A}$ rated output of the unit). Future versions of the firmware will store this value in static RAM so this power-on value can be modified by the control system.

In case of a fault that results in current above the set point, the fault protection system is triggered. The output pulse is immediately terminated (within 50uS), a "crowbar" switch is placed across the output, the high voltage DC power supply is disconnected, and the internal capacitor bank is discharged though a series of "dump" resistors in the pulser chassis. A fault indicator lights on the front panel and fault code is made available to the control system. The pulser may not be re-enabled until the fault condition has been cleared.

\section{Diode Voltage}

The operator has no control of this parameter. A V/I curve for each tile is measured by the control system and stored in a database. The operating voltage expected for any requested current is known from this data base. A "trip" voltage two volts below this value is automatically selected. If more than a single diode bar fails this lower limit will be exceeded and a fault as described above will occur. The amount of "Offset" from the stored V/l curve may be changed by an administrative user as above. An upper limit of $150 \mathrm{~V}$ is built into the DEI firmware. This protection feature defends both the pulser itself as well as the diode load. If many diodes bars in an array should short the pulser would be asked to dissipate too much power possibly resulting in failure of the pulser output switches.

Additionally, the output voltage during the interpulse period is monitored (nominally $V_{\text {out }}$ and $I_{\text {uut }}$ are zero during this period). If this voltage exceeds $10 \%$ of the nominal diode "on" voltage above, a fault condition is generated. Voltage output during this time period indicates a pulser fault that is causing $\mathrm{CW}$ current to flow in the load. 


\section{Pulse Width}

As with diode current. the control system software will prevent the operator from requesting a pulse width outside the sate operating region. A "gated" mode in which the width of the triggered pulse determines the output pulse width is available but not used. As configured for Mercury. the pulser is edge triggered and an onboard digital counter determines the output pulse width. The DEI tirmware has a hard limit of $2 \mathrm{~ms}$. This parameter will also be changed to static RAM in future units. As an additional protection the trigger distribution chassis limits (in hardware) the input pulse to lus. So that in the unlikely event that a pulser was placed in the gated mode. only a very short (and therefore safe) pulse could be generated.

\section{Pulse Repetition Rate (PRF)}

The control system software will prevent the operator from requesting a repetition rate outside the sate operating region. PRF is limited in the Mercury system by hardware in the trigger regeneration chassis. A timed gate rejects any repetition rates greater than $1 \mathrm{OHz}$. Currently there are no firmware limits to PRF in the pulsers. Future units will include a programmable gate to limit maximum repetition rates.

Table 5. Summary of laser diode protection system.

\begin{tabular}{|l|l|l|l|l||}
\hline PARAMETER & OPERATOR & SOFTWARE & FIRMWARE & TRIGGER CHASSIS \\
\hline $\begin{array}{l}\text { values set by the } \\
\text { operator }\end{array}$ & $\begin{array}{l}\text { values limited } \\
\text { by the control } \\
\text { system software }\end{array}$ & $\begin{array}{l}\text { values limited } \\
\text { inside the pulser } \\
\text { chassis }\end{array}$ & $\begin{array}{l}\text { values limited inside the } \\
\text { trigger regeneration } \\
\text { chassis }\end{array}$ \\
\hline Diode Current & $\mathbf{X}$ & $\mathbf{X}$ & $\mathbf{X}$ & \\
\hline Diode Voltage & & $\mathbf{X}$ & $\mathbf{X}$ & $\mathbf{X}$ \\
\hline Pulse Width & $\mathbf{X}$ & $\mathbf{X}$ & $\mathbf{X}$ & $\mathbf{X}$ \\
\hline Repetition Rate & $\mathbf{X}$ & $\mathbf{X}$ & $*$ & \\
\hline
\end{tabular}

*future upgrade/retrofit

\section{Electrical Efficiency}

The efficiency of the pulsers at the nominal operating point is about $8+\%$, and improves to about $91 \%$ at maximum output. Repetition rate has little effect on efficiency $(1-2 \%$ improvement at $20 \mathrm{~Hz})$. At the nominal operating point the overall electrical efficiency is about $78 \%$ (Table 6 ). These calculations are based on the following assumptions: 1) The high voltage DC to the pulsers will be supplied by an external switching power supply with a $A C$ line to DC output efficiency of $82 \%-88 \%$. 2) A minimum of $10 \mathrm{~V}$ "head room" must be maintained across the switching elements (FETs) during the output pulse to assure control stability. 3) The each pulser will have a capacitor bank of 35,000uF.

Table 6. Summary of electrical etficiencies.

\begin{tabular}{|l|l|l|}
\hline Operating Point & Nominal & Maximum Output \\
\hline Diode Voltage & $80 \mathrm{~V}$ & $90 \mathrm{~V}$ \\
\hline Diode Current & $70 \mathrm{~A}$ & $130 \mathrm{~A}$ \\
\hline Pulse Width & $750 \mu \mathrm{s}$ & $1.5 \mathrm{~ms}$ \\
\hline Pulser Efficiency & $\approx \mathbf{8 4 \%}$ & $\approx 91 \%$ \\
\hline $\begin{array}{l}\text { Power Supply } \\
\text { Efficiency }\end{array}$ & $\approx 84 \%$ & $\approx 87 \%$ \\
\hline Overall Efficiency & $\approx 71 \%$ & $\approx 79 \%$ \\
\hline
\end{tabular}


There are two primary sources of lost energy in the pulser. The first is due to capacitor bank droop (Area A in Fig. 58). In order for the pulser to maintain a constant current out during the pulse as the capacitor bank droops more voltage must be dropped across the FETs at the beginning of the pulse. Making the capacitor bank larger can decrease this loss. but only small improvements in efficiency are obtained for relatively large capital costs in capacitors.

The second (and major) source of lost power for the pulsers comes from FET headroom (Area B in Fig. 58). At the nominal operating point 0.525 joules per shot are lost. however at the maximum output the loss increases to $3 \mathrm{~J} / \mathrm{shot}$. Overall efficiency at the nominal operating point can be improved by about $1.3 \%$ per volt of reduced headroom. Experiments conducted using an absolute minimum of headroom (i.e. the pulse just begins to droop) have shown pulser efficiencies greater than $95 \%$ at the nominal operating point. This is not a realistic operating condition for the pulser but it does demonstrate that the numbers quoted in the chart above are conservative.

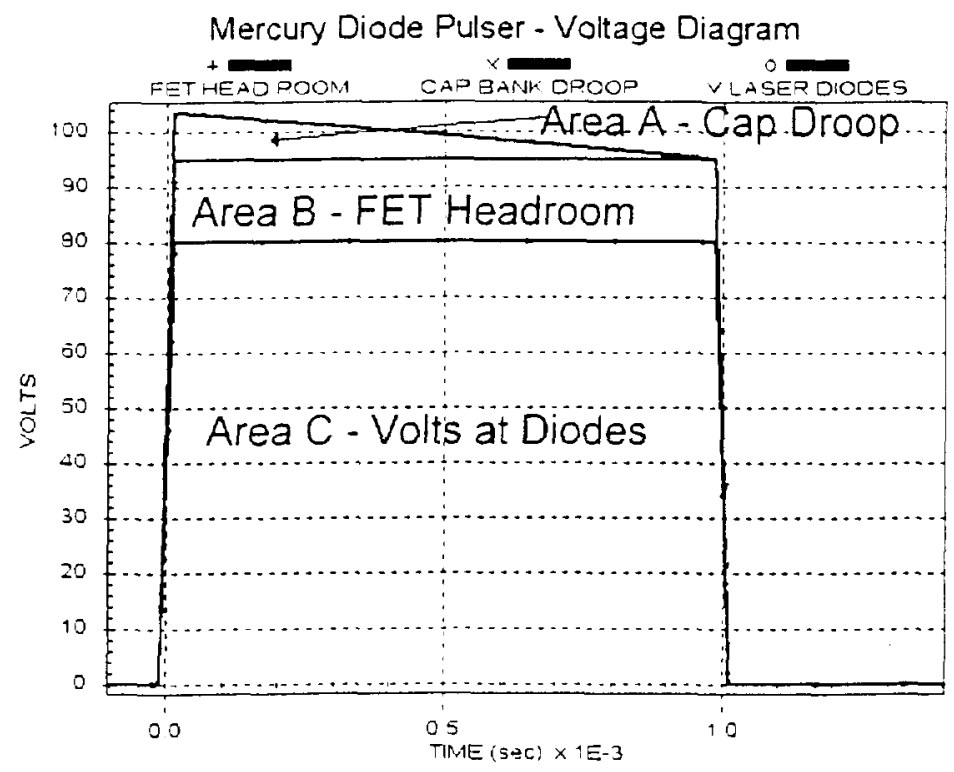

Fig 58. Description of electrical efficiency headroom

\section{Future Development Areas}

Two major areas for pulsed power development for DPSSLs are overall electrical efficiency and the integration of the drive electronics to the diode pump arrays. The most important performance parameter associated with the pulsed power for the pump arrays is electrical efficiency. Current state of the art drivers are based on an analog current regulator in conjunction with a switch mode DC power supply. The overall efficiency of this type of combination is limited to about 80 percent. A more integrated approach that uses pulse width modulation and the elimination of the separate DC supply should be capable of efficiencies in the 90 to $95 \%$ range. Effectively the switch mode power supply would become the pulser.

Future DPSSL systems feature very closely packed arrays of diode modules. As an example. the Mercury Laser will have four groups of diode modules, each requiring up to $48 \mathrm{~kW}$ average electrical power. with a frontal area of only 5.3 by 12 inches. Using current techniques, this would require at least four six foot equipment cabinets for each array. Clearly, a more integrated approach is required. The next step in integration will be the development of Hybrid MicroCircuits (HMC) in which switching, current 
sensors. control circuitry and diode protection all reside in a single compact module. The goal will be a pulse power package with the same frontal area as the laser diode module it drives. Improvements in electrical efficiency noted above should also reduce thermal management issues resulting in more compact systems. The resulting reduction in both volume and weight would be particularly important to defense applications. 


\section{Computer Controls}

The architecture of the Mercury Laser necessitated that an automatic control system be developed to meet the needs of the system. In its final configuration the Mercury Laser will have $1+0$ diode pulsers. 20 DC power supplies. + timing control boxes. + diode cooling systems. 2 slab gas cooling systems. and several diagnostic systems. A block diagram of the Mercury laser from the controls perspective is shown in Fig. 59. The control system is also responsible for personnel and equipment satety on a day-to-day basis. The control system was designed to be tlexible and easily adaptable as the laser development evolves.

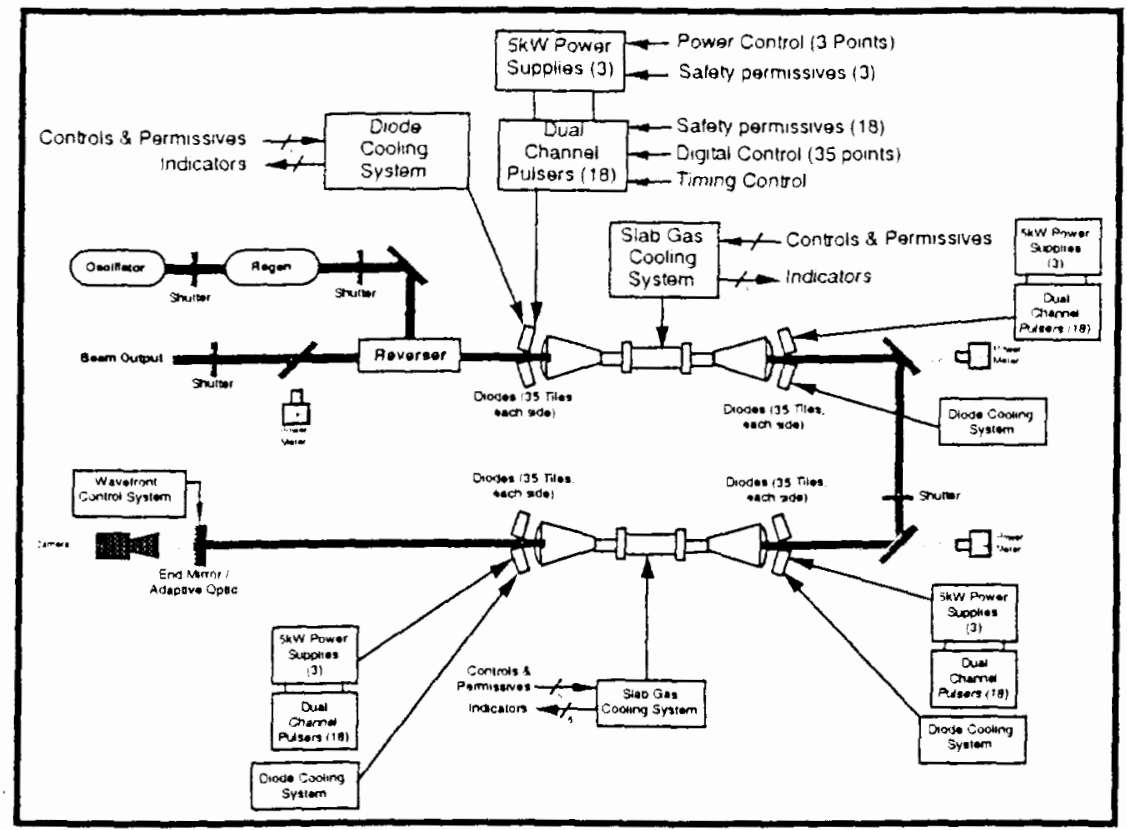

Fig. 59 Controls block diagram.

\section{System Hardware Architecture}

A block diagram of the Mercury control system hardware architecture is shown in Fig. 60. Figs. 61 and 62 highlight the PLC in the overall architecture of the control system. The control system utilizes a computer and programmable logic controller (PLC). These two devices are connected via a proprietary bus with an VO card located in the computer. The computer is tasked with controlling the power to the diodes by controlling the amplitude and duration of current sent to the diodes. The PLC is tasked with control of the diode cooling systems. glass slab cooling systems. and with personnel \& equipment safety. The status of the PLC is monitored by the computer system and the PLC accepts commands from the computer system.

The PLC controls and monitors the personnel safety interlocks which includes doors into the laboratory, equipment cabinet doors. as well as other safety monitors as required. The PLC provides permissives to the power supplies and pulsers when requested by the control computer provided that all interlocks are in the safe condition. The PLC removes the permissives to the power supplies and pulsers whenever a condition occurs that could present a personnel hazard or a condition that would harm the equipment. An example of a personnel hazard is if a laboratory door were to be opened without the proper access code being provided. An example of a condition that could cause harm to the equipment would be if the cooling system operated outside of its preset limits. 


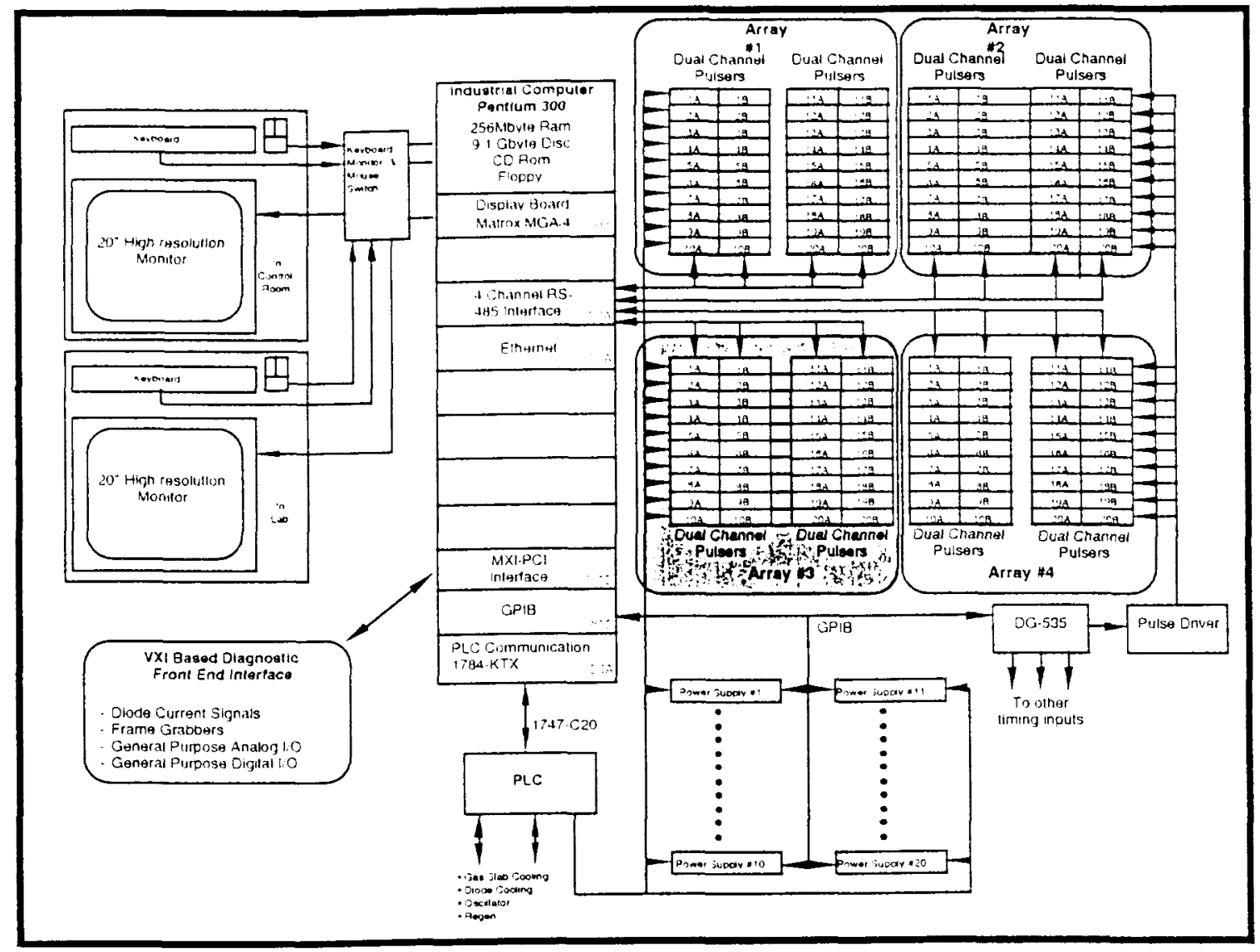

Fig. 60 Mercury control system hardware architecture.

\section{System Software Architecture}

A block diagram of the Mercury Control System Software Architecture is shown above. The software in the control computer is written in LabVIEW. version 5.1. The software consists of three main modules: 1) PLC interface: 2) Power control; and 3) Power monitor. At the completion of Phase 1 the first two modules have been written and are being used to operate the Mercury Laser. Asynchronous communication between the major sottware modules is done using Queues and Globals.

The system was designed to control $1+0$ diode pulsers. 20 power supplies. and 4 timing control boxes. One portion of the power control screen contains the command buttons used to control the major functions of the control system: the pulser configuration. power supply configuration. and timing configuration. Another section of the control screen is used to control and display status of the 140 pulsers, up to 35 at a time. The bottom of the display provides status information for the power supplies. which are connected to the pulsers being displayed. The configuration of the system that defines the connections between power supplies. pulsers. and diode arrays is logged in an EXCEL data file that is loaded at the beginning of the control program. Multiple configurations are available in this file to allow for multiple experimental setups. 


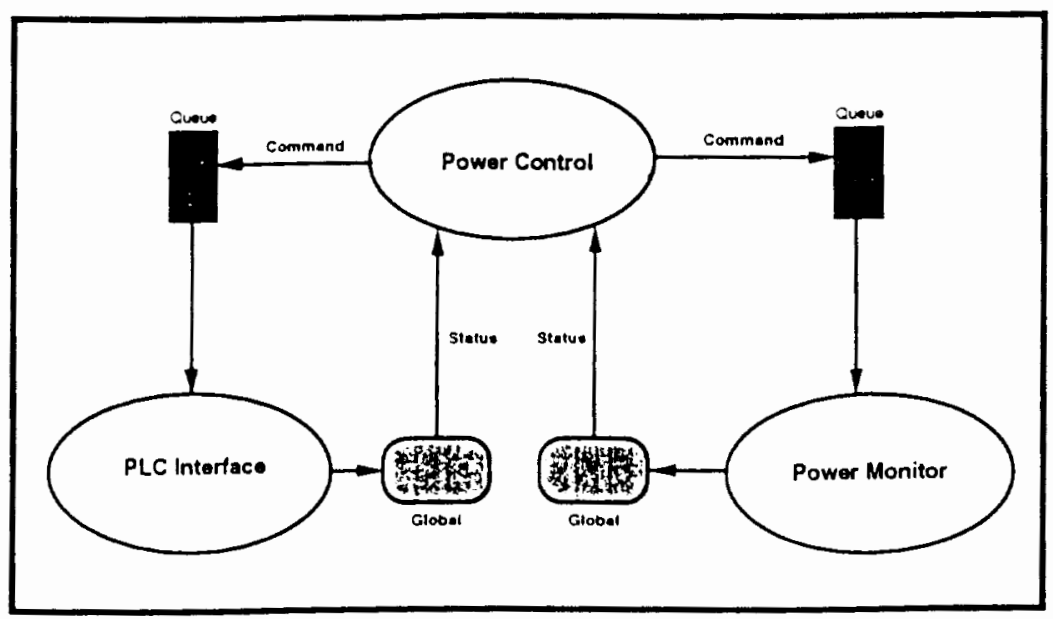

Fig. 61 PLC block diagram

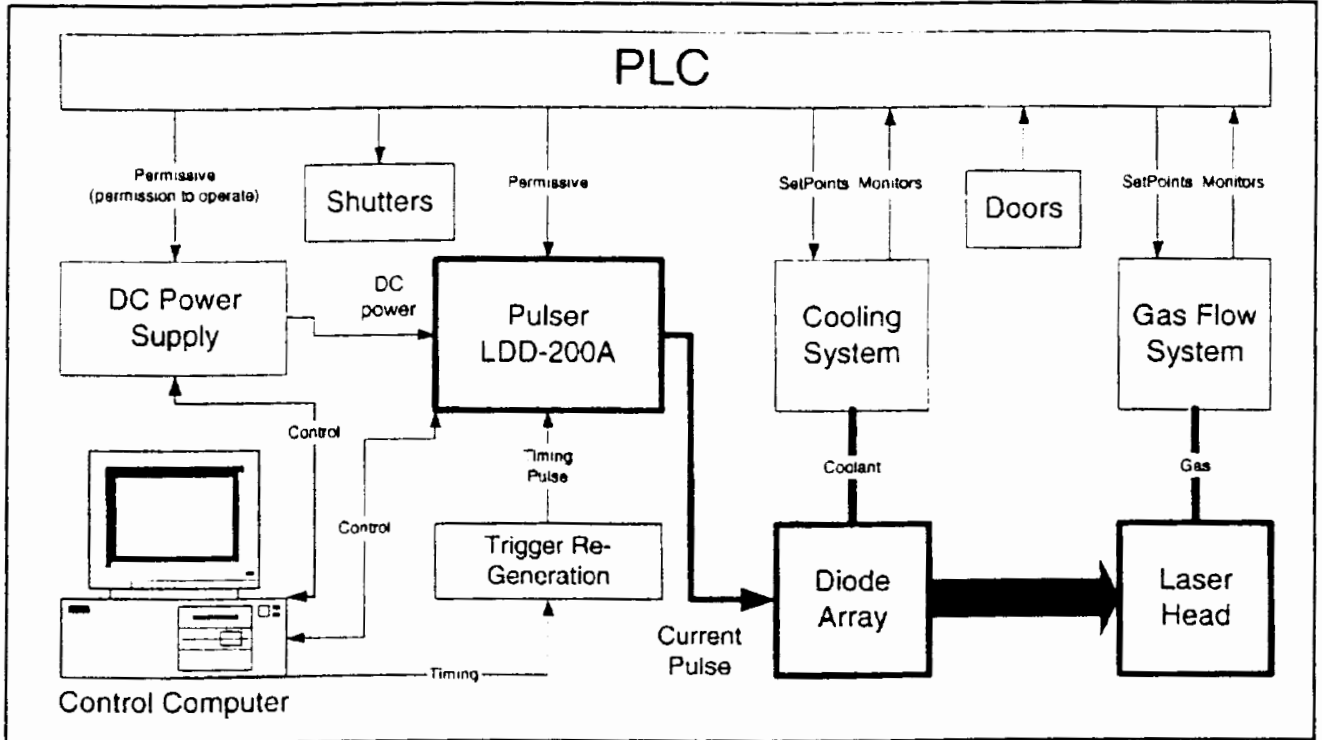

Fig. 62 PLC block diagram

\section{Future Activities}

The actual current \& voltage being applied to each diode array is not currently monitored. The original plan was to query each laser diode pulser for this information and provide a real time display. However, the current hardware and data acquisition system are incompatible for high speed, high quantity data retrieval \& display. Future activities will include the development of an independent monitor for the current \& voltage being applied to each array. A scanning A/D converter will be mounted in a VXI chassis to sample current and voltage for each pulse for each array. This information will then be displayed on the control front panel. The current and voltage as well as shot count on each diode array needs to be archived in order to support long-term analysis of the performance of the system. This will also be developed in the next phase of the project. 


\section{System Utilities}

The Mercury Laboratory $\left(1680 \mathrm{ft}^{2}\right)$ consists of two separate rooms: the laser/target laboratory and the control room. which are located in the west end of Building 381. Two of the original rooms in the building were recontigured into one larger room in order to provide adequate space for the laser system and power supplies (see Fig. 63) A new insulated inner wall was added on both the south and west sides of the room to provide better than \pm 1 " $\mathrm{C}$ thermal stability. The upper ceiling above the future laser/target room was also insulated to improve temperature stability.

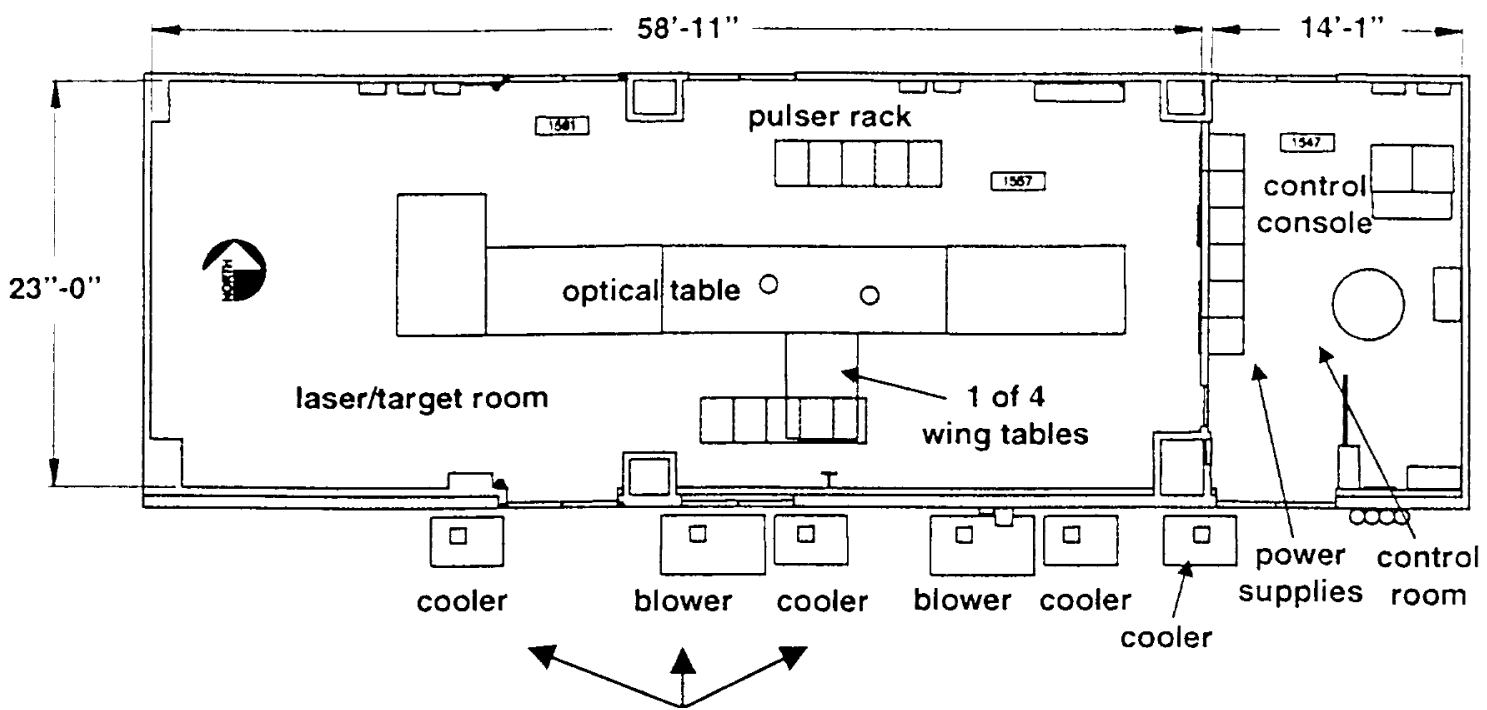

Utilities currently installed

Fig. 63 Mercury Floor Plan

An important issue with any high power laser system is cleanliness of the surrounding environment. Dirty optics and dust floating in the beam can result in decreased power, poor beam quality. and damage to optics. A new ceiling with HEPA filtration was installed in the facility to keep the environment clean to better than a class 10,000 rating. The existing air conditioning system was modified to supply cool air into the plenum area above the room ceiling.
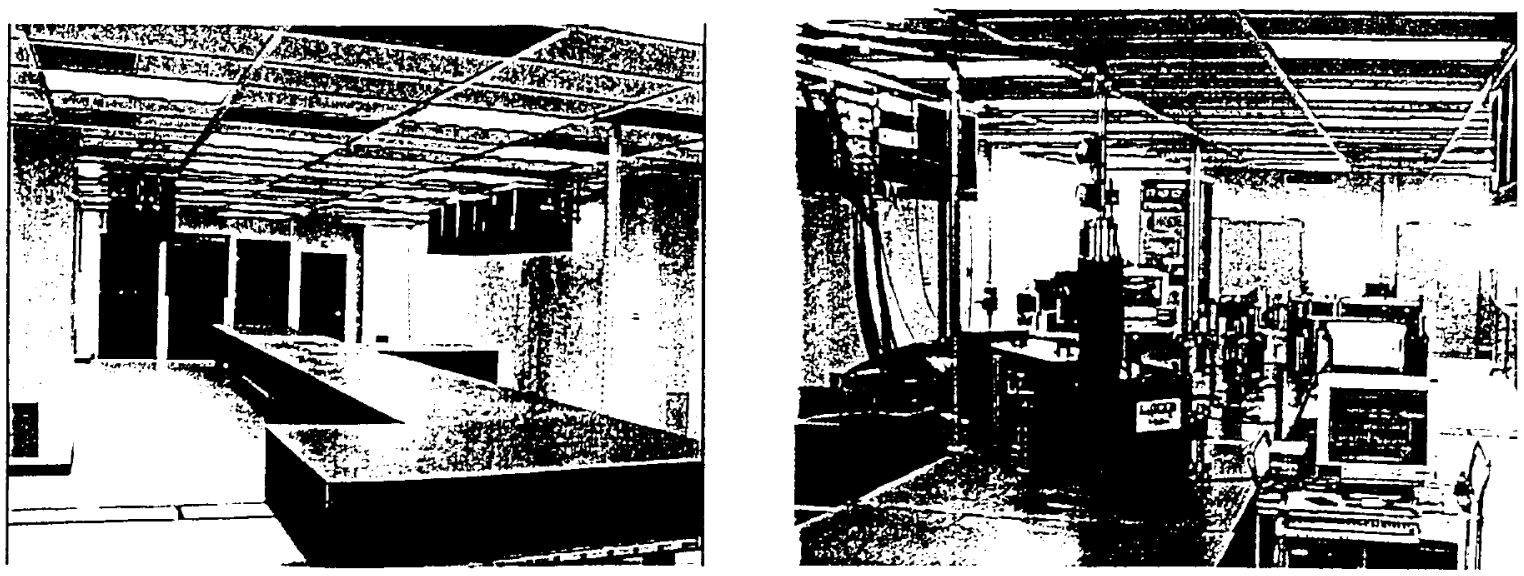

Fig. 64 Mercury Facility at completion of room modification phase (left) and after adding hardware. 
HEPA filters were installed above the optical tables so as to provide an adequate level of clean air in the laser laboratory (<class 10.000). Return air ducts were located close to the room walls. This filtration system was designed to provide a circular air pattern from the center of the room ceiling downward and toward the outer walls.

The electronics racks for the control and pulse power system were installed as a wall between the laser/target room and the control room to provide easy access. The racks also act as a protective barrier. preventing laser exposure in the control room. Racks for locating the diode pulsers were mounted from the ceiling grid in the laser lab to minimize space issues. The special equipment for cooling the laser diodes and slab amplifiers was installed on the outside of the building. under a protective awning. As shown in Fig. 64. cable trays were installed to link the electronics racks with the pulser racks, the special equipment outside of the lab. and the Programmable Logic Controller (PLC) that controls the system interlocks.

Cooling systems for the laser diode arrays and laser amplifiers constitute the bulk of the required utilities for the facility. Due to the high heat load generated by operation of the laser diodes, the diode arrays were designed to be liquid cooled. Table 7 contains the general specifications for cooling the laser diode arrays. The table lists both the nominal as well as the maximum expected requirements for normal operation. A liquid coolant other than water was required in order to allow for low temperature operation and temperature tuning of the diodes. Coolants such as Fluorinert or Galden are commonly used in laser diode cooling systems since they have outstanding heat transfer properties. The cost and availability issues of these fluids required us to use an alternative coolant. A new tluid class from $3 \mathrm{M}$. Hydrofluoroethers. or HFEs, had properties that were very similar to the Fluorinert fluids. however the cost/gallon was significantly lower and it was more environmentally friendly. As a result, HFE-7100 was chosen as the coolant for use in the laser diode chillers.

Table 7. Cooling specifications for each laser diode array

\begin{tabular}{|l|l|l|}
\hline & Nominal & Maximum \\
\hline Heat Capacity $(\mathrm{kW})$ & 2.4 & 4.0 \\
\hline Temperature Range $\left({ }^{\circ} \mathrm{C}\right)$ & -40 to +20 & \\
\hline $\begin{array}{l}\text { Temperature Stability } \\
\left({ }^{\circ} \mathrm{C}\right)\end{array}$ & $+1-0.3$ & \\
\hline Flow Rate (GPM) & 26 & 32 \\
\hline Pressure (PSI) & 47 & 70 \\
\hline Available Power & $480 \mathrm{~V}, \quad 80 \mathrm{~A}$, & \\
\hline Case Dimensions & $3 \varnothing$ & $\begin{array}{l}60 " \mathrm{w} \\
48 \text { x } 48 " \mathrm{~d}\end{array}$ \\
\hline
\end{tabular}




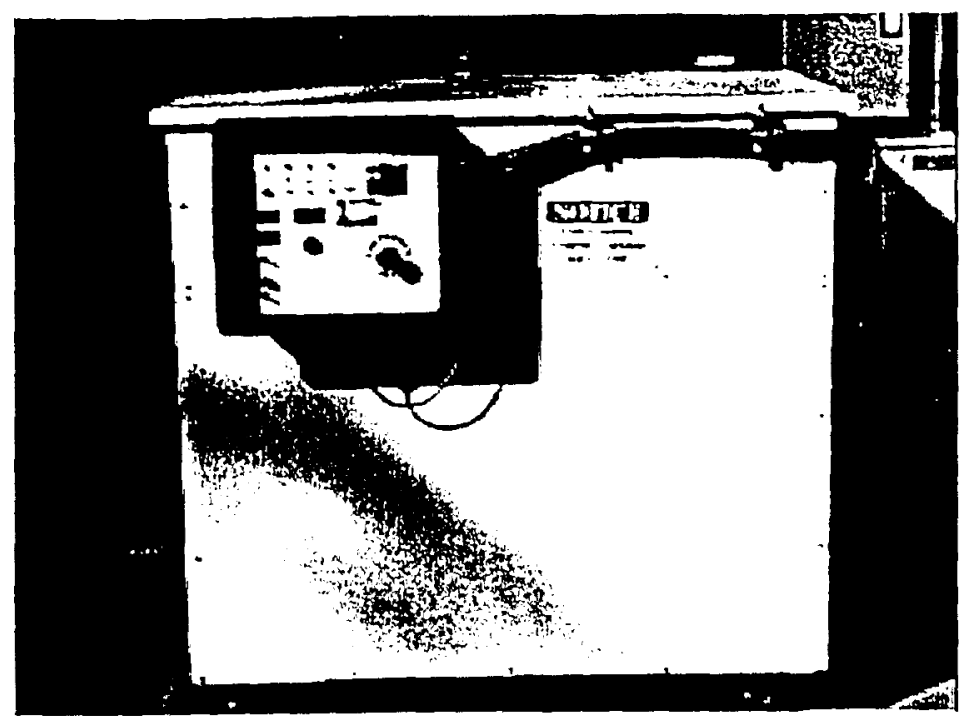

Fig. 65 Diode array chiller.

A custom engineered Bay Voltex chiller was selected for this application (Fig 65). Two of the required four units were procured and one was installed for testing the first laser diode array. The unit provides $22-26 \mathrm{GPM}$ of coolant to the array at $65 \mathrm{PSI}$ and operates from -50 to $+20^{\circ} \mathrm{C}$. The unit typically operates at $208 \mathrm{~V}, 52 \mathrm{~A}$. 30 . The use of alternative fluids such as HFE-7100 required compatible materials within the diode chiller as well as the supply and return lines to the diode array. Thread seals were noted as a potential problem area (leaks), and hose materials with elastomers were to be avoided (static charge storage). Stainless steel, brass and copper were selected for the bulk of the construction materials, with small lengths of Teflon/EPDM hose to be used to minimize pump-induced vibrations. The copper and brass pipe and fittings were brazed to obtain leak-tight connections, while stainless steel tubing was employed in the supply and return lines and utilized compression type fittings to prevent leaks

Table 2 lists the specifications for the gas blower/cooler manufactured by Wes-Co Industries of Oakland, California. The first of two systems was procured, installed and tested during FY99. The system, shown in Fig. 66, is in a 72"w x 44"d x 84"h frame, and contains a variable speed blower with variable frequency drive, water cooled chiller, gas to water heat exchanger, ULPA filter, and proportional cooling control valve. The system was connected to the slab amplifier cell by way of 2 " stainless steel tubing with compression type tittings.

In summary, the construction phase of the facility is complete. Minor modifications to the facility may be required if there are significant changes to the optical system. Installation and activation of the experimental hardware has occurred. 
Table 8. Gas blower/cooler specifications

\begin{tabular}{|c|c|c|}
\hline Feature & Specification & Intertace \\
\hline Gas till & Helium. $100 \%$ & \\
\hline Gas tlow rate (CFMI) & $80-150(320-600 \mathrm{sctm}+1-0.5)$ & 0 -10VDC output \\
\hline Blower control & Variable: off to full speed & $0-10 \mathrm{VDC}$ input \\
\hline $\begin{array}{l}\text { Desired maximum volume } \\
\text { of equipment }\left(\mathrm{ft}^{3}\right)\end{array}$ & 6 & \\
\hline Cooling capacity & $1.5 \times(0.5 \mathrm{~kW}+$ blower heat load $)$ & \\
\hline Gas temperature range $(" \mathrm{C})$ & $15-25$ & $0-10$ VDC input \\
\hline Gas temperature stability ("C) & $+1-0.3$ & \\
\hline Gas temperature monitor (") $\mathrm{C})$ & $0-40+1-0.5$ & $0-10$ VDC out \\
\hline $\begin{array}{l}\text { Cooler temperature monitor } \\
(" \mathrm{C})\end{array}$ & $0-30$ or greater & $0-10$ VDC output \\
\hline Particulate filtration & ULPA $(99.9999 \%$ at $0.003 \mathrm{~m})$ & \\
\hline Pressure range (PSIG) & $15-45$ & \\
\hline Pressure test & 90 PSIG & \\
\hline Vacuum test & 1 Torr & \\
\hline Available power & $208 \mathrm{~V}, 100 \mathrm{~A}, 30$ & \\
\hline
\end{tabular}

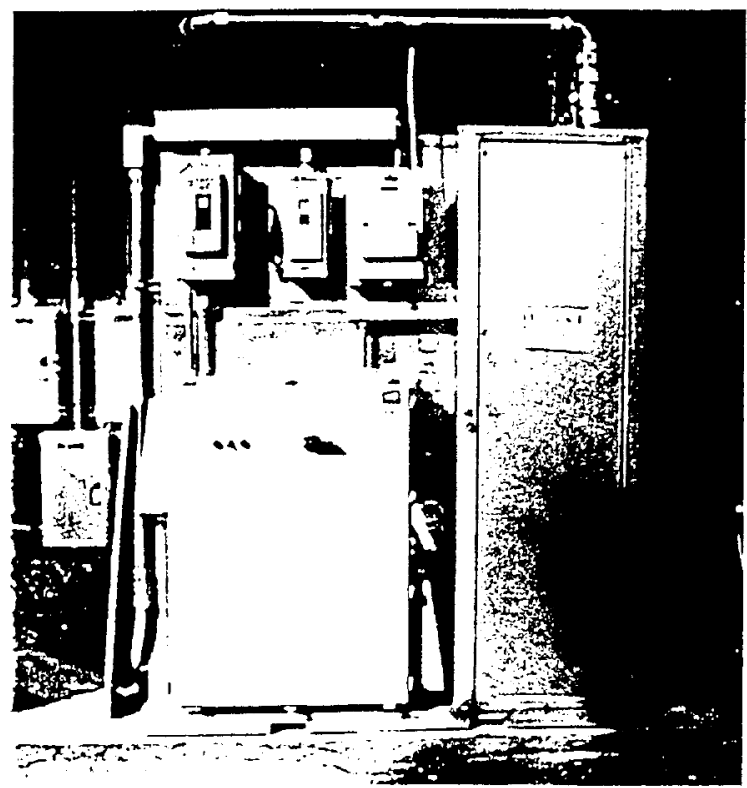

Fig. 66 Gas blower/recirculator 


\section{System Modeling}

\section{A. System Energetics}

We have developed a baseline performance code to model energy tlow in the Mercury laser system. This modeling code. written in BASIC. was developed to pertorm quick optimization and tradeoff studies to help zero in on a system design before two dimensional. detailed propagation codes were exercised. To keep the analysis manageable. several approximations and simplifications have been made in describing the pump excitation and laser energy extraction physics as detailed below.

To begin. we model the laser diode pump array performance including the effects of transient heating during the laser diode pump pulse. Transient diode heating is accounted for by dynamically tracking the roll-off in diode array output power. and the red shift in the diode emission spectrum. with increasing temperature during the pulse. In addition to the contribution of diode chirp to the pump spectrum. the entire array is characterized by a Gaussian distribution of wavelengths representing the spread in the center wavelengths of the approximately 6400 individual diode bars that will comprise the entire pump array. The peak power capability of the array is $6+0 \mathrm{~kW}$. The following equation is used to describe the temporal and spectral dependence of the diode array output energy

$$
\frac{\partial^{2} E}{\partial t \partial \lambda}=\frac{P(t)}{\sqrt{\pi} \lambda_{1 / t t w W}} e^{-\left(\frac{\lambda-\lambda_{1}(t)}{\lambda_{1, r t w}}\right)^{2}}
$$

where $\lambda_{\text {letrw }}$ is the 1/e spectral halfwidth that describes the pump array radiation and $\lambda_{0}(\mathrm{t})$ is the instantaneous center wavelength of the pump pulse at time t. A thermal diffusion model that is anchored to experimental data is used to describe the transient temperature rise experienced by the diode array during a pump pulse. Calling $\Delta \mathrm{T}(\mathrm{t})$ the transient temperature rise of the diodes during a pump pulse. the instantaneous central wavelength of the diode array, $\lambda_{0}(t)$, and the instantaneous optical power developed by the array, $P(t)$, are given by

$$
\lambda_{i}(t)=\lambda_{(0)}+\frac{d \lambda}{d T} \Delta T(t)
$$

and

$$
P(t)=P_{0}(1-\beta \Delta T(t))
$$

respectively. where $\mathrm{d} \lambda / \mathrm{dT}$ and $\beta$ are constants that characterize the diode bars used in the pump array. The ransient temperature rise is given by

$$
\Delta T(t)=\alpha \sqrt{t}
$$

where $\alpha$ is a constant characteristic of the diffusion properties of the silicon $V$-basis backplane the diode bars are bonded to. The values of the various constants that describe the diode pump array in the modeling that follows are as given in Table 9. 
Table 9 Parameter values used in diode pump pulse model.

\begin{tabular}{|l|l|}
\hline$\lambda_{\text {1/chlW }}$ & $4.8 \mathrm{~nm}$ \\
\hline$P_{01}$ & $640 \mathrm{~kW}$ \\
\hline $\mathrm{d} \lambda / \mathrm{dT}$ & $0.3 \mathrm{~nm} /{ }^{\circ} \mathrm{C}$ \\
\hline$\alpha$ & $632^{\circ} \mathrm{C} / \mathrm{sec}^{1 / 2}$ \\
\hline$\beta$ & $0.00251 /{ }^{\circ} \mathrm{C}$ \\
\hline
\end{tabular}

As described in an earlier section, the diode pump radiation is delivered from the diode array to the laser slabs using a combination of optical conditioning on the laser diode bars and non-imaging transport through a ducted delivery system. In conjunction with this ray trace modeling code. a 1 dimensional pump pulse propagation model has been developed. This propagation code dynamically calculates the gain loading of the individual laser slabs using a treatment that includes the effects of $\mathrm{Yb}^{\text {it }}$ ground state depletion. spectrally selective pump wave depletion and $\mathrm{Yb}^{3+}$ fluorescence decay. The impact of amplified spontaneous emission (ASE) and radiation trapping in the laser slabs is also included in the model using a simplified treatment previously developed and presented by C.D. Orth et al. ${ }^{31}$. The following equations are used to model the double-ended pump excitation on the slabs.

$$
\begin{aligned}
& \frac{\partial I_{r}(z, t)}{\partial z}+\frac{n}{c} \frac{\partial I_{r}(z, t)}{\partial t}=I_{r} \sigma_{p}\left[\left(f_{a}^{p}+f_{b}^{p}\right) n_{2}-f_{a}^{p} n_{0}\right] \\
& -\frac{\partial I_{l}(z, t)}{\partial}+\frac{n}{c} \frac{\partial I_{l}(z, t)}{\partial t}=I_{l} \sigma_{p}\left[\left(f_{a}^{p}+f_{b}^{p}\right) n_{2}-f_{a}^{p} n_{0}\right] \\
& \frac{\partial n_{2}(z, t)}{\partial t}=-\frac{1}{h v_{p}}\left[I_{r}(z, t)+I_{l}(z, t)\right] \sigma_{p}\left[\left(f_{a}^{p}+f_{b}^{p}\right) n_{2}-f_{a}^{p} n_{0}\right]-\frac{n_{2}(z, t)}{\tau(t)}
\end{aligned}
$$

Where $I_{i}$ and $I_{r}$ represent the left and right propagating pump waves, respectively, $n$ is the refractive index of S-FAP, $\mathrm{n}_{2}$ is the $\mathrm{Yb}^{3+}{ }^{2} \mathrm{~F}_{5 / 2}$ excited manifold number density, $\sigma_{p}$ is the spectroscopic pump absorption cross section, and $\mathrm{f}_{\mathrm{a}}^{\mathrm{p}}$ and $\mathrm{f}_{\mathrm{b}}^{\mathrm{p}}$ are the ${ }^{2} \mathrm{~F}_{7 / 2}$ and ${ }^{2} \mathrm{~F}_{5 / 2}$ Boltzmann occupation factors of the initial and final Stark levels coupled by the pump radiation, respectively. The output of this 1 -dimensional code is used to normalize the 2-dimensional energy deposition profiles calculated using our ray trace code. As an example of what is done, Fig. 66 depicts the gain loading in one of the slabs. The assumed cross sectional dimensions of the slabs are $4 \mathrm{~cm}$ by $6 \mathrm{~cm}$ and they are $0.75 \mathrm{~cm}$ thick. Only the central $3 \mathrm{~cm}$ by $5 \mathrm{~cm}$ is excited by the pump beam leaving a $5 \mathrm{~mm}$ skirt around the perimeter of the slab to allow the slab edge cladding to be spatially separated, and somewhat insulated, from the high intensity pump radiation.

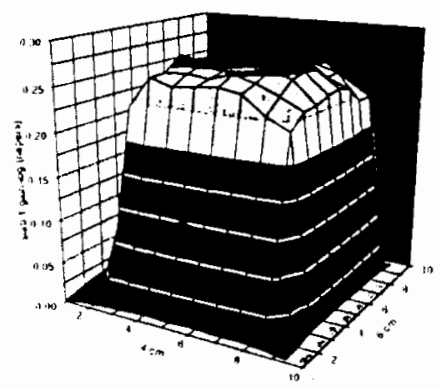

Fig. 66. 2-D pump deposition profile of one of the Yb:S-FAP slabs that is calculated using our 3-D ray trace code with its output normalized to our 1-D pump excitation code.

Our pump propagation code contains a 1-D analysis that more fully describes the physics than is done with our 3-D ray trace code. For example the $1-D$ code includes spectral profiles of the pump that 
are dynamically updated as it is propagated through the stack of slabs. as well as the spectral protiles of the $\mathrm{Yb}^{3+}: \mathrm{S}-\mathrm{FAP}$ absorption. The spectral profile of the generated pump radiation is calculated using the model described above in equations (1) through (4). This ability to model the pump radiation and its absorption in the slabs in some detail has turned out to be an important lever in the optimization of the laser system. primarily due to the relatively narrow pump absorption line in the vicinity of $900 \mathrm{~nm}$ that is being utilized in the Yb:S-FAP excitation as shown in Fig. 67. This narrow absorption feature critically determines the center wavelength of the laser diode array required for system optimization.

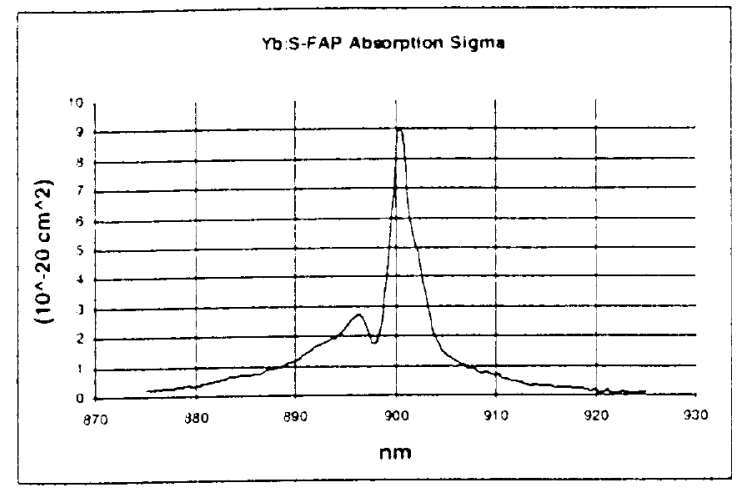

Fig. 67 The spectrally resolved room temperature absorption cross section of $\mathrm{Yb}: \mathrm{S}$-FAP in the vicinity of the $900 \mathrm{~nm}$ feature that will be used for excitation

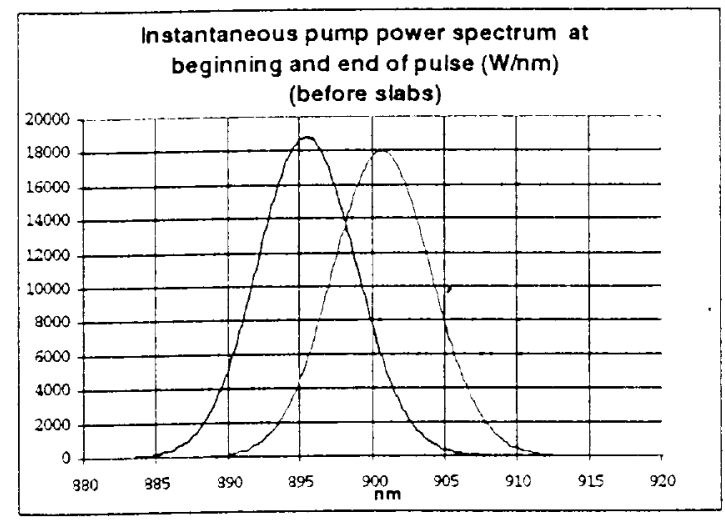

Fig. 68 Plots of the modeled instantaneous pump power spectrum at the beginning and end of a 750 usec pump pulse and at the diode array. The diode spectral modeling includes the impact of chirp during the pulse as well as droop. both the result of the transient heating of the diode bar during a pulse.

As an example of how our modeling tracks the dynamic development of the laser pump excitation pulse. Fig. 68 depicts the instantaneous pump power spectra generated by the laser diode array at both the beginning and end of a $750 \mu \mathrm{sec}$ long pump pulse for an array with optimized wavelength. In accordance with the previously discussed model. there is both a red shift and a roll-off in output power evident between the beginning and the end of the pulse. Fig. 69 shows one impact of the narrow absorption feature on the pump excitation radiation. The integrated pump power spectrum after the slabs exhibits a spectral hole burned into it by the absorption of the $\mathrm{Yb}^{3+}:$ S-FAP. Because one of the driving design considerations of the laser system is the optimization of its efficiency, the developed code has been very useful in specifying desired diode operating characteristics as well as performing system sensitivity studies of these diode characteristics. 


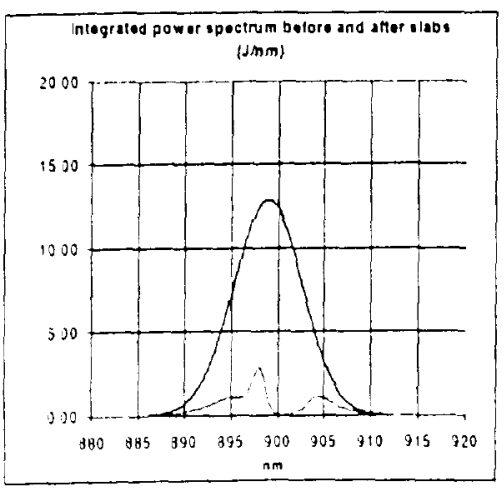

Fig. 69 Plots of the integrated pump power spectrum prior to entering the first slab and after exiting the last slab. The impact of the narrow $\mathrm{Yb}$ :S-FAP absorption feature is evidenced by the spectral hole in the emerging pump radiation.

One of the issues that we have addressed with our modeling capability is the specification of the slab-to-slab dopings to both optimize laser performance and keep the thermal load from slab to slab balanced. The balancing of the thermal load from slab to slab is an important consideration for the optimum performance of the gas cooling being employed in the system. By using three different doping values of $1.4 \times 10^{19} / \mathrm{cm}^{3}, 1.7 \times 10^{19} / \mathrm{cm}^{3}$, and $2 \times 10^{19} / \mathrm{cm}^{3}$, as shown in Fig. 70 it is possible to hold the thermal loading from slab to slab to better than $10 \%$ as shown in Fig. 71 . Using the stress fracture parameter value of $1.2 \mathrm{~W} / \mathrm{cm}$ appropriate for $\mathrm{Yb}^{3+}: \mathrm{S}-\mathrm{FAP}$, the laser slabs are projected to be at $11 \%$ of fracture with the system operating at full average power $(10 \mathrm{~Hz})$.

In addition to modeling the average thermal loading of the Yb:S-FAP slabs we have also looked at the time resolved thermal loading of individual slabs during the application of a pump excitation pulse. Fig. 72 depicts this time resolved thermal loading for the first slab in the stack (slab number 1 in Figs 69 and 70). This time resolved profile was calculated to assess the impact of the thermal shock imparted to the slab during a single pump excitation pulse, which could for example lead to ringing in the slab and impact beam quality as a result. Looking at the deposition profile in Fig. 71, it is seen that the thermal load actually increases slightly during the first $50 \mu \mathrm{sec}$ of the pulse as a result of the chirping diode wavelength. At the beginning of the pump pulse, the wavelength of the diode is slightly to the blue side of the Yb:S-FAP peak absorption feature, but this feature is traversed due to the red shifting of the diodes as they dynamically heat up during a pump pulse. The slow roll off of the thermal deposition after the first $100 \mu \mathrm{sec}$ is due to a combination of the diode wavelength continuing to red shift past the peak absorption feature, a droop in the actual diode power output during the pulse, and a drop in the absorption of the slab due to ground state depletion.

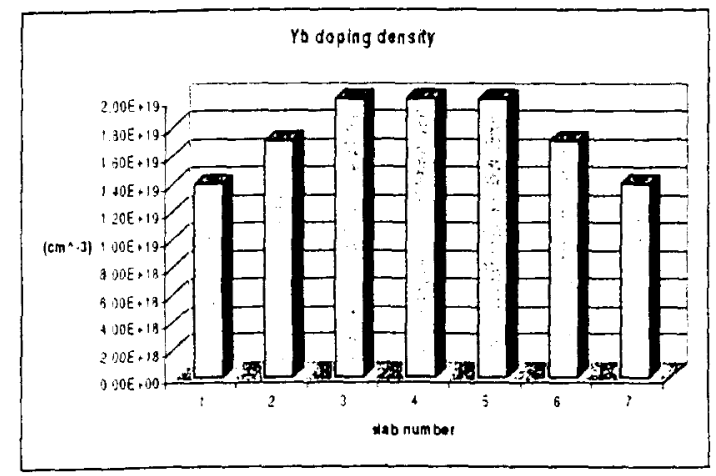

Fig. 70 The doping density of the slabs is feathered from stack center to edge slab to maintain a near uniform thermal loading from slab to slab in the presence of pump depletion 


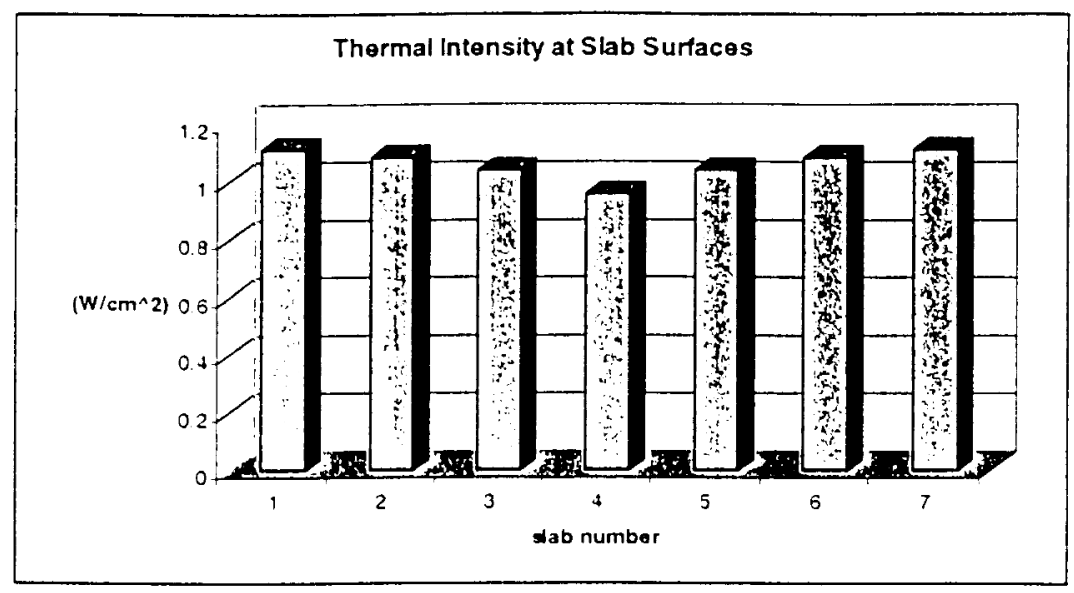

Fig. 71 The feathered doping from slab to slab permits the thermal loading of the individual slabs to be held within $\pm 10 \%$ of each other in the presence of strong pump wave depletion.

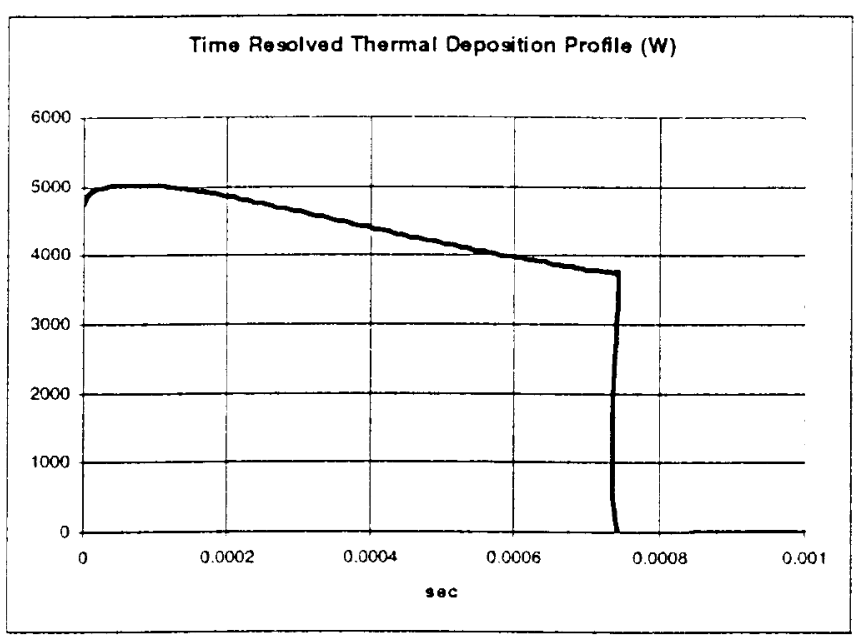

Fig. 72 The time resolved thermal deposition profile for the first slab in the stack. Time resolved profiles were modeled to assess issues such as potential ringing in the slabs as a result of the thermal shock during a single pulse

Energy extraction modeling uses a standard Frantz-Nodvik analysis that has been modified to account for the quasi-three level nature of the $\mathrm{Yb}^{3+}$ ion to calculate the gain-log, $\mathrm{g}_{\mathrm{i}}$, and saturation fluence. $\Gamma_{i}$, after each pass through the system.

$$
\begin{aligned}
& g_{i}=-\ln \left[1-e^{-\Gamma_{i-1} / \Gamma_{s a a}}\left(1-e^{-\xi_{i-1}}\right)\right] \\
& \Gamma_{i}=\Gamma_{s a i} \ln \left[1+e^{g_{a}}\left(e^{\Gamma_{t-1} / \Gamma_{s a t}}-1\right)\right]
\end{aligned}
$$

where 


$$
\begin{aligned}
& g=\left[\left(f_{b}^{e}+f_{b}^{e}\right) n_{2}-f_{c}^{e} n_{b}\right] \sigma_{e m}^{\ell} \\
& \Gamma_{s a t}=\frac{1}{f_{c}^{e}+f_{b}^{e}} \frac{h v_{e}}{\sigma_{e m}}
\end{aligned}
$$

In (7). $\sigma_{\mathrm{em}}$ is the spectroscopic emission cross section of the $10+7 \mathrm{~nm} \mathrm{Yb:S-FAP} \mathrm{line,} \mathrm{which} \mathrm{has} \mathrm{a} \mathrm{value}$ of $6 \times 10^{-11} \mathrm{~cm}^{2}$ and $\mathrm{f}_{\mathrm{d}} \mathrm{c}^{\circ}$ and $\mathrm{t}_{\mathrm{h}}{ }^{\circ}$ are the ${ }^{2} \mathrm{~F}_{7 / 2}$ and ${ }^{2} \mathrm{~F}_{5 / 2}$ Boltzmann occupation factors of the Stark levels coupled by the laser radiation. respectively. Fig. 73 shows the extracting pulse fluence and the net gainlog of the $1+$ slabs used in the Mercury amplifiers as a function of the pass number during the four-pass extraction. The final slab to be traversed by the extracting pulse is projected to see a tluence of $9 \mathrm{~J} / \mathrm{cm}^{2}$. underscoring the importance of high damage threshold optics and optical coatings. As a result of the ground state absorption present in $\mathrm{Yb}^{3+}: S-F A P$, not all of the stored laser energy can be extracted. This can be understood by examining the definition of $\mathrm{g}$ (the gain) in eq. (7). Examining this definition. it is seen that a certain amount of population inversion must be present just to reach optical transparency at the laser wavelength. For the presently considered Mercury system. this minimum stored energy required to reach transparency is $23 \mathrm{~J}$. which represents $12 \%$ of the $190 \mathrm{~J}$ of total stored energy at the time of extraction. In addition to tracking the extracting fluence and the gain-log as function of pass number, the code also performs a path integral of the local extraction pulse irradiance. assuming temporally square extracting pulses and ignoring pulse shape distortions due to gain saturation. This path integral is then used to calculate a B-integral for the system, which for an assumed 10 nsec duration extraction pulse is displayed in Fig. 74.
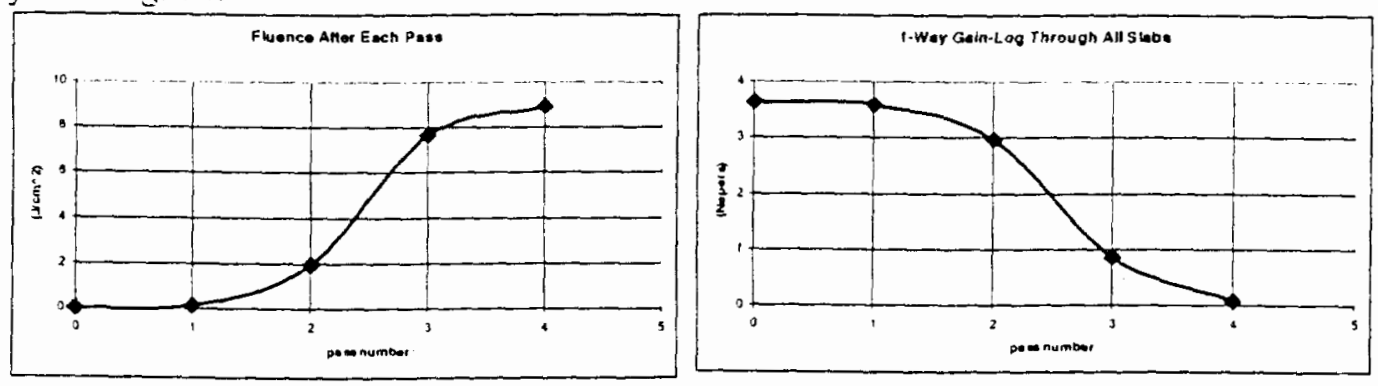

Fig. 73. Extracting tluence and net gain-log of the 14 slabs that comprise the amplifier system as a function of the pass number during the four-pass extraction.

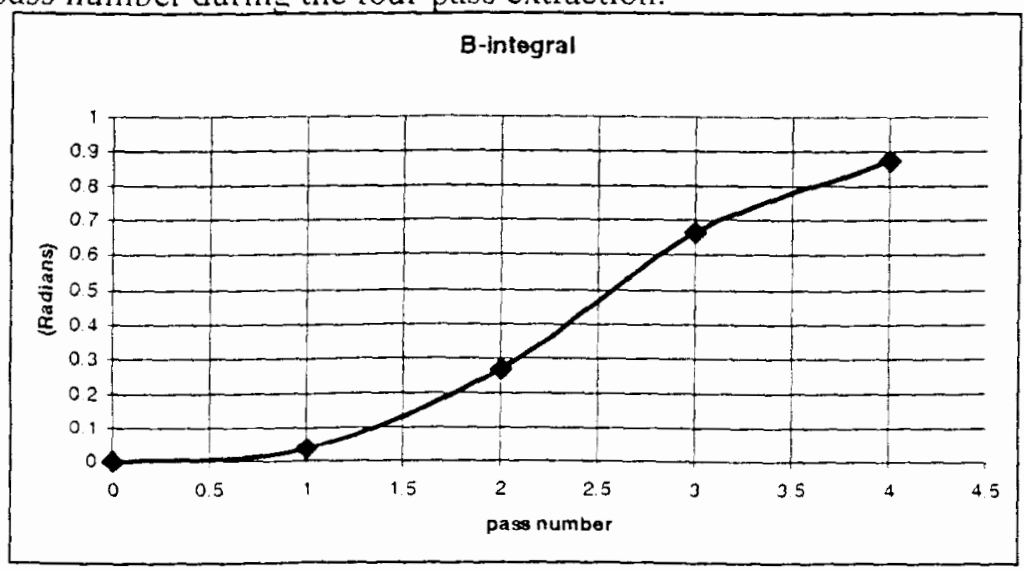

Fig. 74. Calculated B-integral for a 10 nsec extraction pulse assuming a temporally square extracting pulses and ignoring pulse shape distortions due to gain saturation 
As already mentioned, one of the motivations for developing our energetics model for the Mercury laser system is to have the ability to perform quick sensitivity and optimizations studies. One example of a sensitivity study is shown in Fig. 75 where both efficiency and output pulse energy are plotted against the pump pulse duration. In terms of pulse energy output and overall efficiency, the goal of the Mercury laser system is $100 \mathrm{~J}$ and a wallplug efficiency of $10 \%$. Using an assumed diode efficiency (electrical-to-optical) of $45 \%$ these goals can be met with a pump pulse duration of $750 \mu \mathrm{sec}$. Another function that can be performed with the energetics code that we have developed is to scope out the energy flow-down in the system. i.e. the various channels in which the generated pump light tlows out of the Mercury laser system. Using the pie chart in Fig. 75 to represent the total optical pump energy developed by the laser diode pump arrays in a $750 \mu \mathrm{sec}$ pump pulse. the breakout of major energy channels is shown.

Yb:S-FAP Mercury Laser

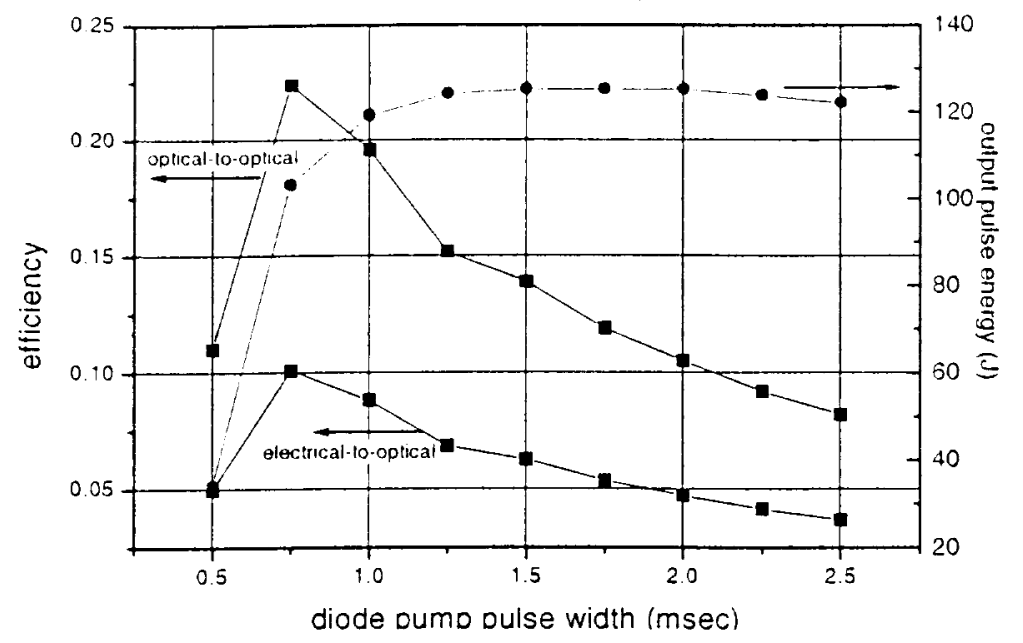

Fig. 75. Mercury laser system performance versus diode pump pulse duration. An assumed diode wall plug efficiency of $45 \%$ (electrical-to-optical) was used in generating the electrical-to-1047 nm optical efficiency curve.

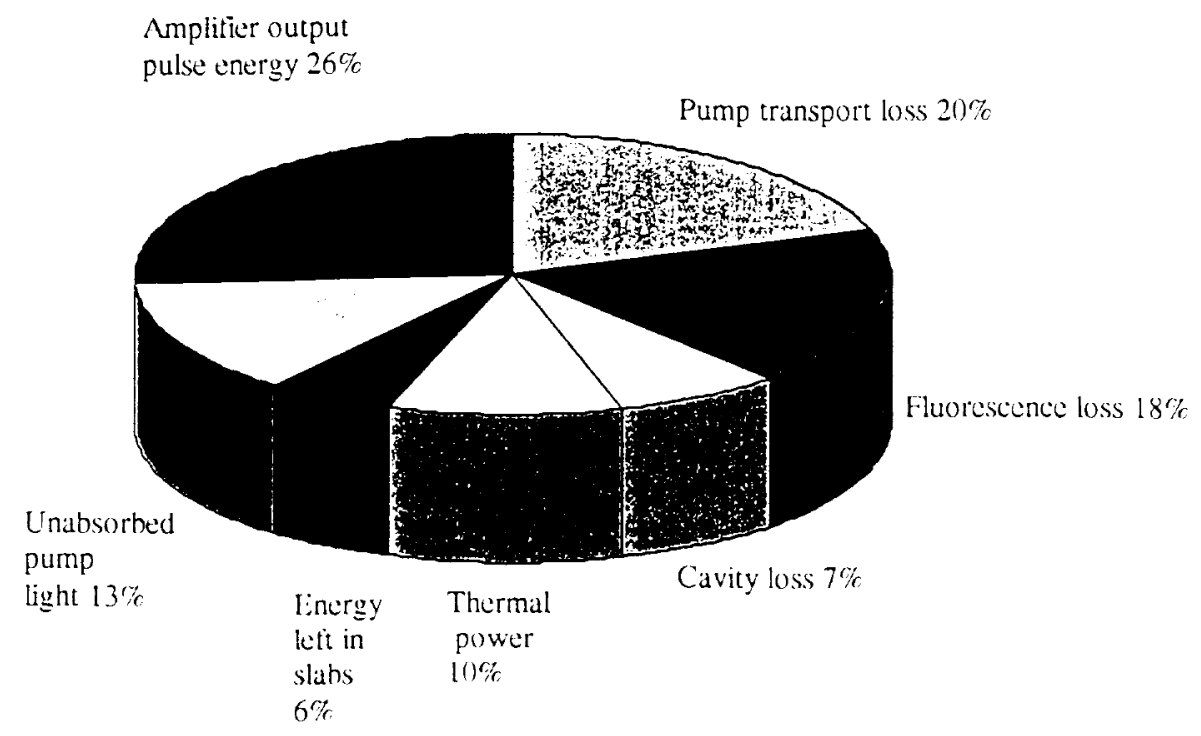

Fig. 76 The energy channels for the pump energy developed in a $750 \mu \mathrm{sec}$ long pump pulse at the end of a pump and extraction cycle. 


\section{B. Beam Smoothing}

We have initiated investigations into the bandwidth capability of the Mercury laser system. Particularly for direct drive applications where irradiation smoothing at a target is important. the ability to generate amplifier output pulses with bandwidths up to several hundred $\mathrm{GHz}$ in the infrared is an important consideration. Due to the relatively narrow emission feature of Yb:S-FAP. as shown in Fig. 77. it is not immediately obvious that a high bandwidth pulse could be efficiently generated in the presence of the strong gain narrowing that will be required to push the amplifier into an efficient mode of operation.

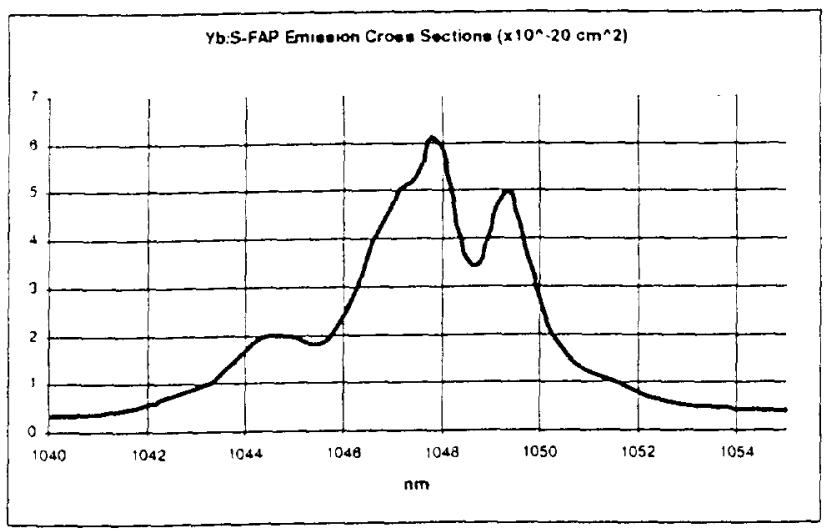

Fig. 77 Spectrally resolved emission cross section of Yb:S-FAP. Note that at a wavelength of $1 \mu \mathrm{m}$. a frequency spread of $0.33 \mathrm{THz}$ (corresponding to $1 \mathrm{THz}$ at the third harmonic) corresponds to $\Delta \lambda=1 \mathrm{~nm}$.

Using the spectral sculpting technique that has been applied to short pulse laser amplification, ${ }^{16}$ it now appears that bandwidths on the order of $0.33 \mathrm{THz}$ are well within the capability of the Mercury amplifier system. As a specific example, and to illustrate the viability of the sculpting technique. we consider the input pulse that would be required to generate an output pulse with a bandwidth of $0.33 \mathrm{THz}$ $(\Delta \lambda=1 \mathrm{~nm})$ centered at the $1047 \mathrm{~nm}$ peak of the Yb:S-FAP emission spectrum. Although there are several choices for the frequency modulation format that could be used, for simplicity we will focus on analyzing a simple linear chirp given by,

$$
v(t)=v_{0}+\beta v_{\text {mod }}\left(2\left[t \cdot v_{\text {mod }} \bmod 1\right]-1\right) .
$$

where $v(t)$ is the instantaneous frequency, and the overall bandwidth of the pulse is given approximately by $2 \beta v_{\text {mod }}$. Taking $v_{\text {mod }}=2 \mathrm{GHz}$ and $\beta=83.3$, for a total bandwidth of $0.33 \mathrm{THz}$, the instantaneous wavelength of the $10 \mathrm{nsec}$ duration extraction pulse that we will consider here chirps over approximately $1 \mathrm{~nm}$, as shown in Fig. 78. The effect of the wavelength chirp depicted in Fig. 78 is to modulate the instantaneous gain cross section seen by the extracting pulse between a value of $5 \times 10^{-20} \mathrm{~cm}^{2}$ and $6 \times 10^{-20}$ $\mathrm{cm}^{2}$, as shown in Fig. 79. If this trequency modulation were applied to a pulse with a flat-in-time intensity profile, then the modulated emission cross section and the resulting modulated gain seen by the chirped pulse would result in the output amplifier pulse being badly amplitude modulated. This is the so called FM to AM problem and is deleterious to the laser amplifier's performance as large AM on the amplifier output pulse can drive the B-integral beyond safe operating limits. However, this is where our new "spectral sculpting" technique can be used to generate an output pulse with a flat temporal amplitude profile in the in the presence of the frequency modulation on the input pulse. Fig. 80 depicts the intensity protile of our linearly chirped input amplifier pulse that is corrected for both square pulsemdistortion (SPD) and FM to AM conversion at the amplifier output. The FM to AM conversion is compensated with this input pulse by adjusting the intensity of the pulse to be relatively high when the instantaneous 
emission cross section. and so gain seen by the pulse. is relatively low and vice-versa. This procedure effectively allows the amplifier output pulse's temporal intensity protile to be free of any AM. Beyond the $2 \mathrm{GHz}$ AM modulation on the input pulse. which is used to compensate FM to AM at the output. the input pulse is seen to have steadily increasing average amplitude with time. This steadily increasing amplitude compensates the gain saturation that occurs during the extraction pulse. and ensures the amplifier output pulse is flat in time without sag.

Fig. 81 shows the frequency spectrum of the linearly chirped input pulse with and without spectral sculpting. As illustrated in Fig. 81. the pulse without spectral sculpting has a that spectral protile. which corresponds to a t7at in time amplitude protile. The reason that this (unsculpted) pulse develops strong AM on passing through the amplifier system is due to the narrow gain feature of the $\mathrm{Yb}$ :S-FAP that preferentially amplifies the central part of the spectrum but not the wings. On the other hand. the spectrally sculpted pulse. which has the AM profile shown in Fig. 80. is suppressed near its center where the gain is high and has higher intensity in its spectral wings where the gain of the Yb:S-FAP amplifier is smaller. The asymmetric shape of the spectrally sculpted pulse in Fig. 81 is due to the asymmetric shape of the gain cross section in the vicinity of its peak. as shown in Fig. 77. Both the spectral protile and the intensity profile of the amplified pulse are shown in Fig. 82. demonstrating the effectiveness of spectral sculpting at eliminating any AM on the amplifier output pulse. Fig. 83 shows the temporally resolved Bintegral accumulated by the pulse as it four passes the Mercury amplifier system. The slight ripple that is evident in the time resolved B-integral is due to contributions made to the B-integral before the AM that is purposely put on the input pulse is smoothed away by the spectrally peaked gain.

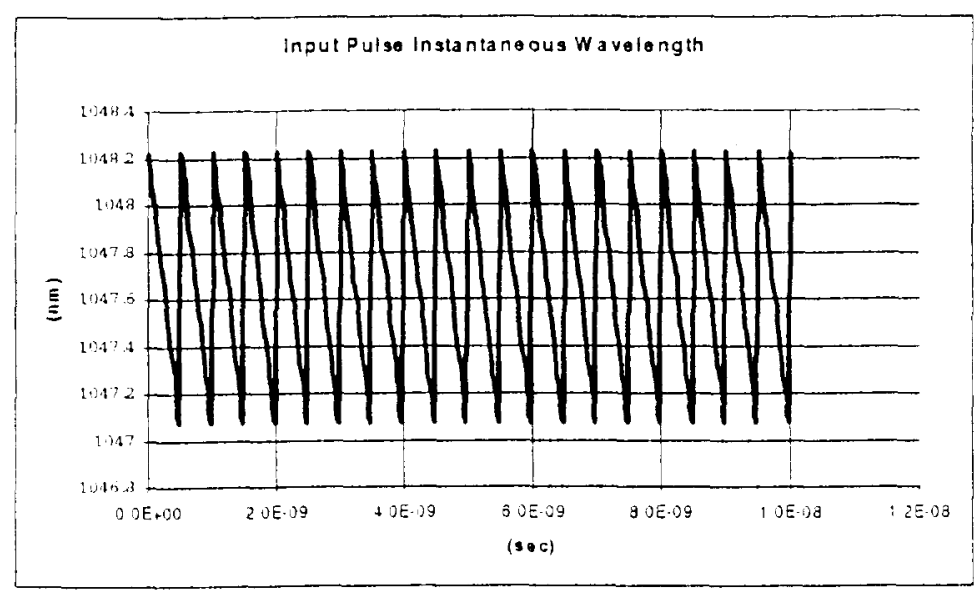

Fig. 78. Instantaneous wavelength of 10 nsec long extraction pulse having a linear chirp as given by eq. (8) with $n_{\bmod }=2 \mathrm{GHz}$ and $\mathrm{b}=83.3$. The pulse is seen to chirp over approximately $1 \mathrm{~nm}$ at the modulation frequency of $2 \mathrm{GHz}$. 


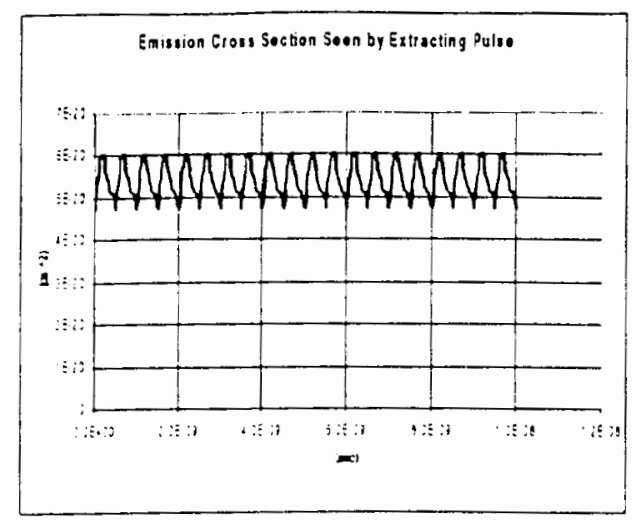

Fig. 79 Instantaneous emission cross section experienced by the chirped extraction pulse.

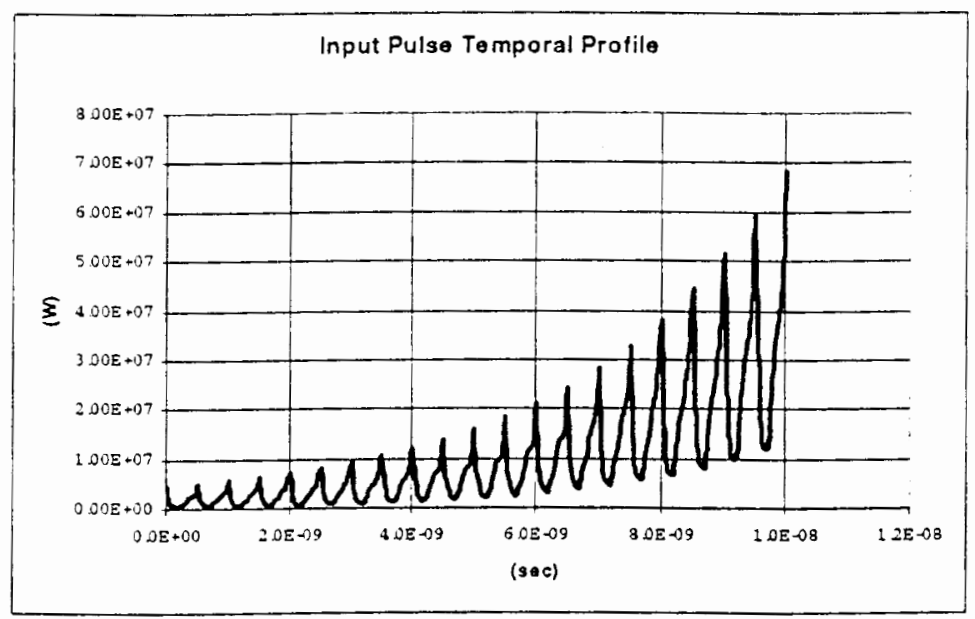

Fig. 80 Input pulse that is spectrally sculpted to suppress FM to AM at the amplifier output.

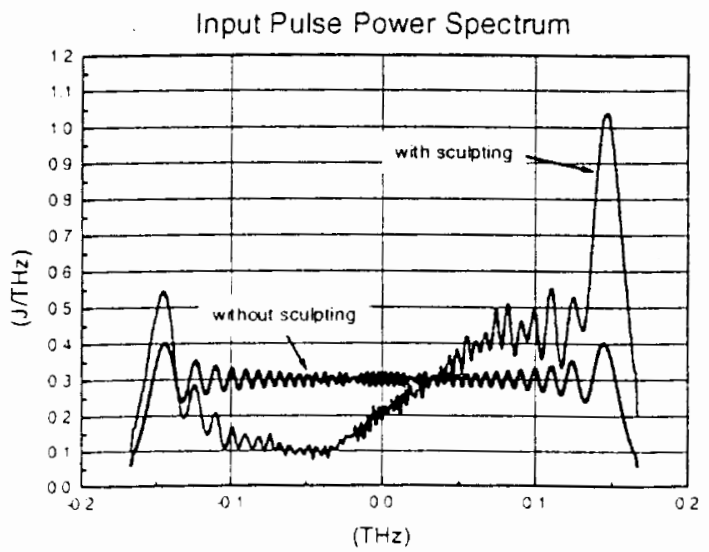

Fig. 81 Frequency spectrum of the linearly chirped input pulse to the amplifier with and without spectral sculpting. The center of the spectrum $(0.0 \mathrm{THz})$ corresponds to a wavelength of $1047.6 \mathrm{lnm}$. 

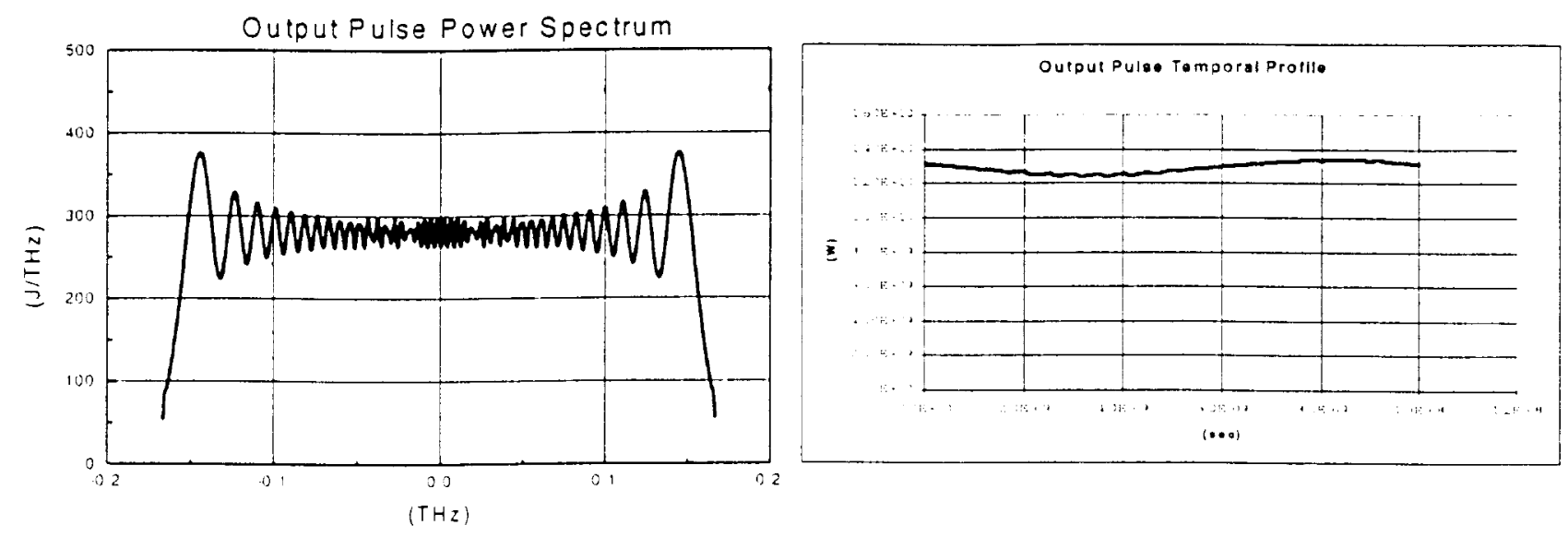

Fig. 82 Spectral and temporal profiles of the Mercury amplifiers output pulse using the spectrally sculpted input pulse as described in the text. In particular. note that the output pulse temporal profile is devoid of any $2 \mathrm{GHz}$ AM which would be present in the absence of sculpting.

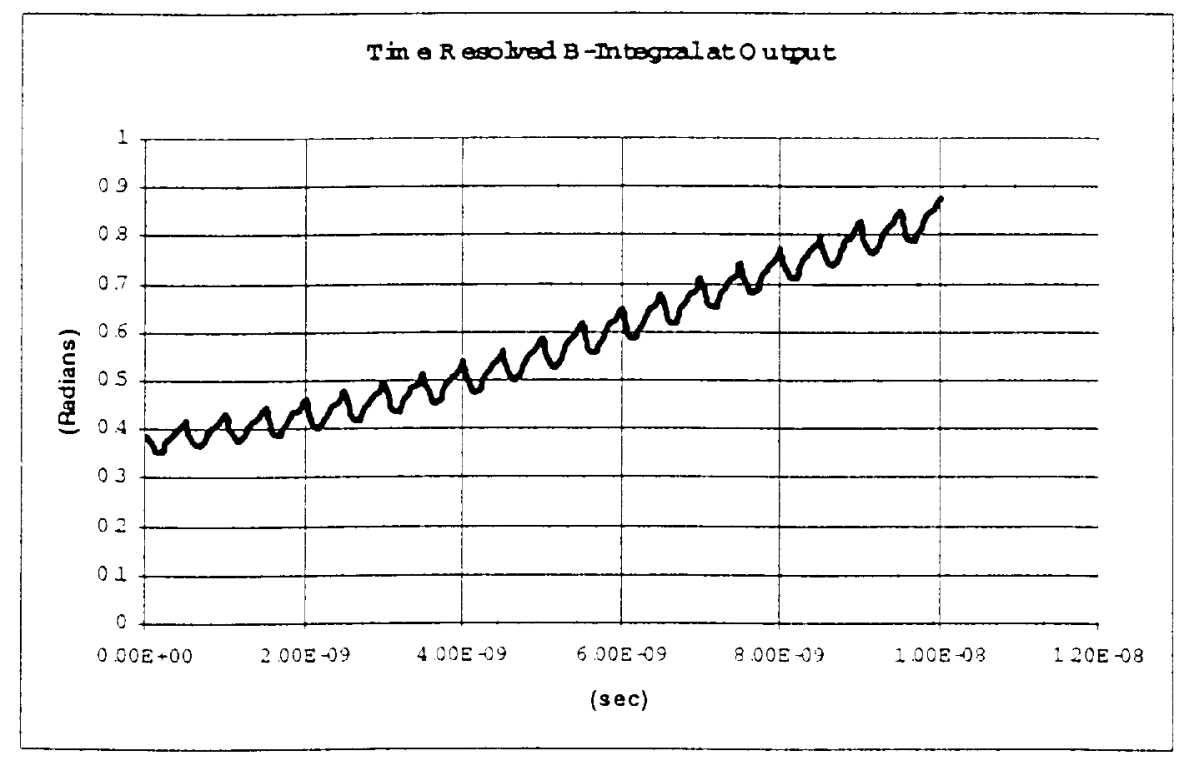

Fig. 83 Time resolved B-integral at the amplifier output. The slight modulation at $2 \mathrm{GHz}$ is due to contribution made during the early stages of extraction before the AM on the input pulse has been smoothed away by the spectrally peaked gain of the Yb:S-FAP. 


\section{Optical Wavefront Distortions}

Detailed calculations of the temperatures, stresses, and optical distortions in the Mercury laser slabs have been performed. This required several solution steps:

(1) Calculation of the thermal source distribution in the slabs and edge cladding

(2) Calculation of the convective boundary condition parameters on the faces of the slab. using the TEXSTAN" boundary layer computer code

(3) Calculation of the temperature distribution in the slab. using the TOPAZ3 ${ }^{18}$ finite element heat transfer code. The boundary and source condition information developed in steps 1 and 2 was applied in this analysis.

(t) Calculation of the thermally induced stresses and displacements using the NIKE3D ${ }^{19}$ mechanics code and the temperature distribution developed in step 3.

(5) Calculation of the optical phase distortion in the slab using the $\mathrm{OPL}^{20}$ code. The calculated temperature (step 3), stress (step t). and displacement (step 4) distributions were used in this analysis.

Through this calculation sequence we are able to precisely account for all pertinent effects on the optical distortion. and properly design the slab and cooling geometry to minimize the distortions. Further details of the calculation procedure are given in Ref. ${ }^{21}$.

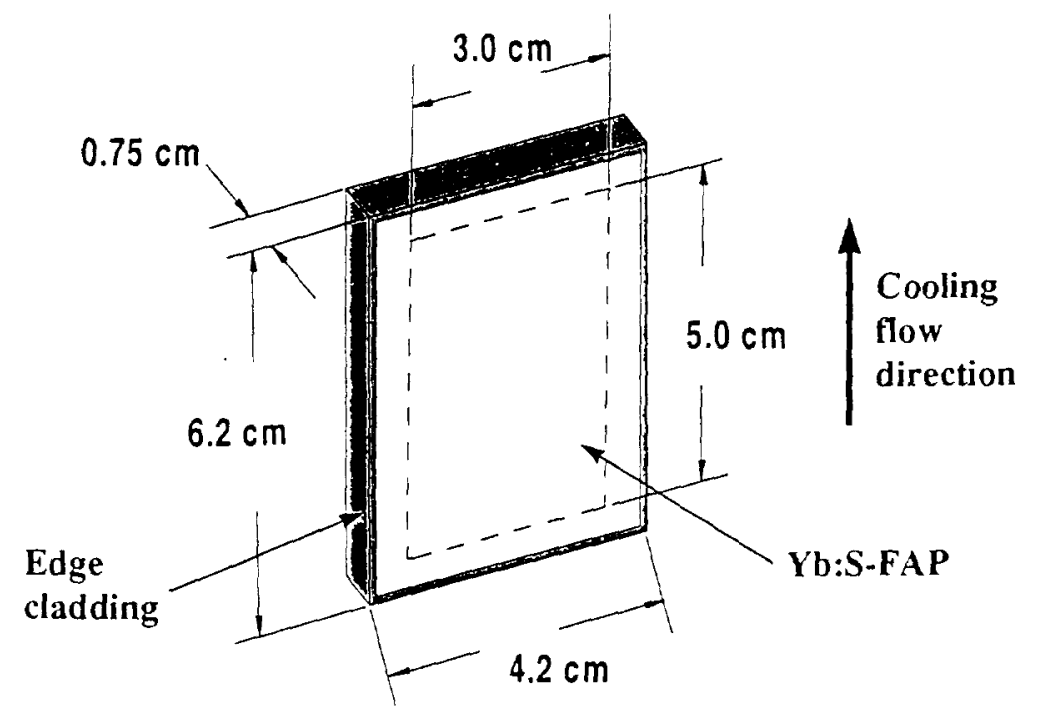

Fig. 84 The slab geometry applied in this analysis. A $0.5 \mathrm{~cm}$ unextracted border rims the $3.0 \mathrm{~cm} \times 5.0 \mathrm{~cm}$ extracted aperture (indicated by the dashed rectangle). The edge cladding is $0.1 \mathrm{~cm}$ thick. 
The slab geometry is shown in Fig. 84. with overall dimensions of $4.2 \mathrm{~cm} \times 6.2 \mathrm{~cm} \times 0.75 \mathrm{~cm}$, an extracted aperture of $3 \mathrm{~cm} \times 5 \mathrm{~cm}$. and an edge cladding thickness of $0.1 \mathrm{~cm}$. The cooling tlow is in the direction of the long dimension of the slab. A representative thermal source distribution is given in Fig. 85. which is extracted trom the calculated pump distribution (see section 10B). The dotted line in Fig. $85 \mathrm{~b}$ denotes the extracted aperture. Note that there is as much as a $50 \%$ roll-off of the source near the edges of the extracted aperture.
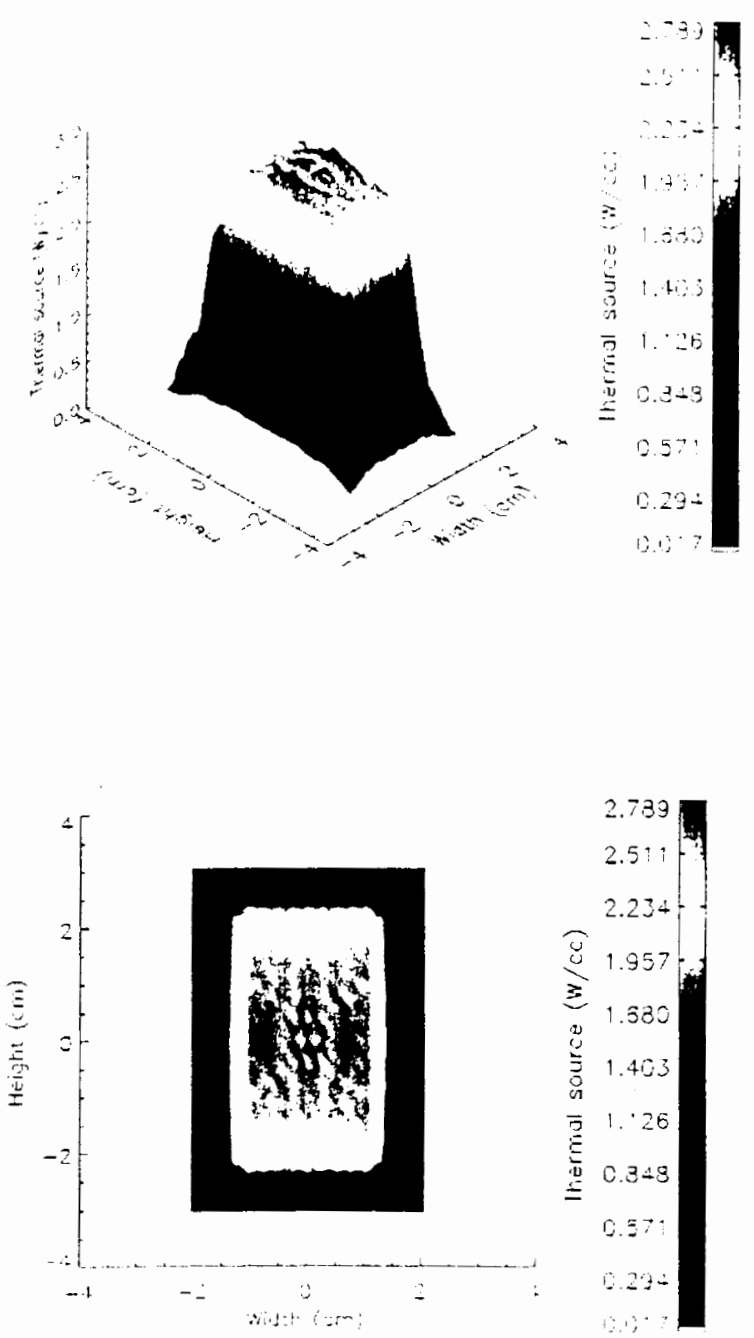

Fig. 85 The thermal source applied in the TOPAZ3D calculation of the temperature distribution in the center slab of a 7-slab stack. The dotted line denotes the extracted portion of the laser slab. Note the rolloff of the source. with a nearly $50 \%$ reduction in intensity between the center and edge of the extracted aperture. (a) a surface plot of the source, (b) a filled-fringe plot of the source. 
The thermal source of Fig. 85 was applied in the TEXSTAN ${ }^{\prime}$ code to evaluate the convective heat transfer coefticient to be applied in the thermal analysis of the slab. Fig. 86 gives plots. across the slab height. of the heat transfer coefficient (Fig. 86a) and the caloric temperature rise of the fluid (Fig. 86b). The perturbations at the edges of the slab are due to variations in the thermal source. The gradual variation down the length of the slab is due to boundary layer development and reduction of the gas density due to heating.
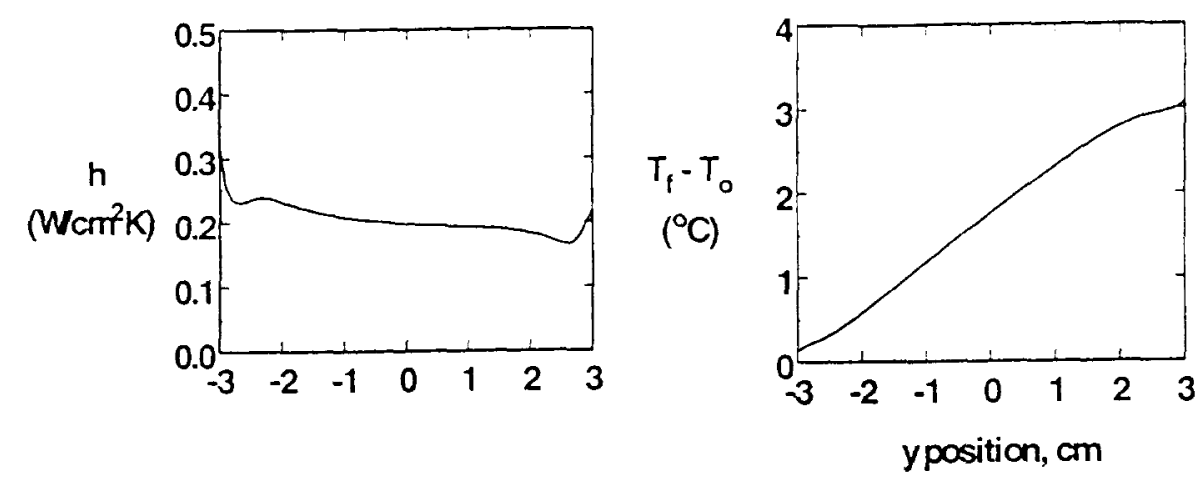

Fig. 86 Plots of the convective heat transfer coefficient (a) and the bulk fluid temperature (b) that were used to describe the convective cooling boundary condition on the large flat faces of the laser slab assembly. Note that the extracted aperture extends from $y=-2.5 \mathrm{~cm}$ to $y=2.5 \mathrm{~cm}$.

(a

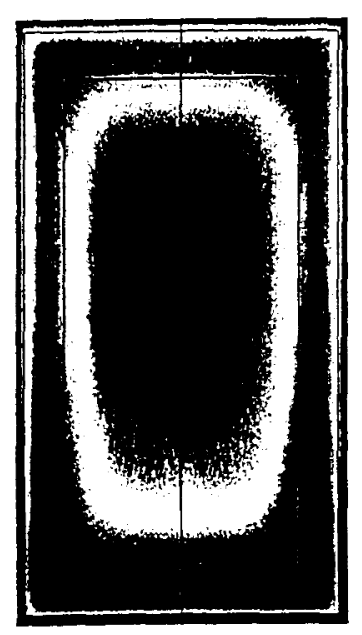

(b

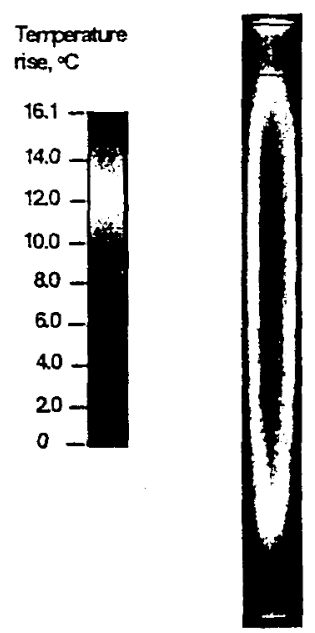

Fig. 87 The temperature distribution in a slab with cooling variations included. All temperature quantities are in ${ }^{\text {}} \mathrm{C}$ temperature rise above the cooling fluid inlet temperature. (a) This view is of a slice taken through the mid-point of the slab thickness, looking in the propagation direction. (b) This view is on slice taken through the mid-width of the slab. The propagation direction in this case is left-to-right. 
The temperature distribution in the slab, relative to the cooling fluid inlet temperature. that results from application of the calculated thermal source distribution (Fig. 85) and convection boundary condition (Fig. 86) is given in Fig. 87. The temperature distribution of Fig. 87a is taken on a plane through the mid-thickness of the slab. The inner rectangular box is the extracted aperture. The roll-off of temperature at the edges of the extracted aperture is due to both a roll-off of the thermal source, and conduction of heat from the hotter regions in the center of the slab to the colder regions at the slab edges that result from the reduced pump intensity. The slight asymmetry from top-to-bottom is due to variations in the heat transfer coefficient as well as the nearly linear increase in the gas temperature as it flows from the bottom-to-top of these figures. Fig. $87 \mathrm{~b}$ gives the temperature distribution on a slice taken through the half-width of the slab. Again we see the roll-off near the edges of the extracted aperture. as well as the convective cooling effects. Also evident in this figure is the center-to-edge temperature gradient through the slab thickness. It is primarily this gradient that generates the thermal stresses.

Total: -0.12 waves

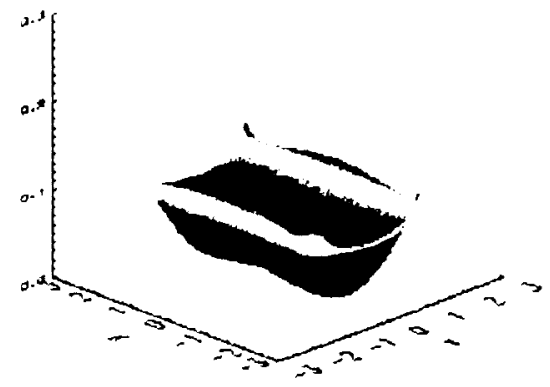

Displacement:

-0.28 waves

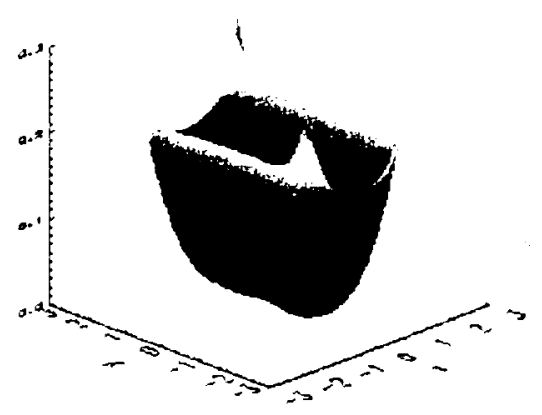

$d n / d T: 0.2$ waves

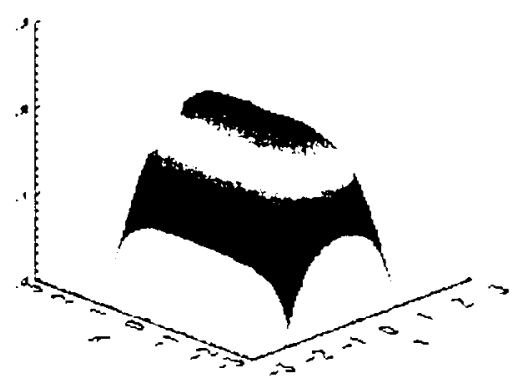

Stress: -0.05 waves

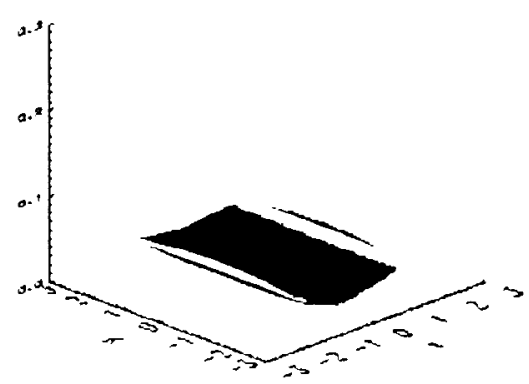

Fig. 88 Decomposition of the optical phase distortion into contributions from temperature $(\mathrm{dn} / \mathrm{dT})$ and stress effects on the index. as well as bulk displacement 
The resulting optical distortions are summarized in Figs. 88 and 89 . Fig. 88 shows the relative contributions of temperature and stress effects on the index of refraction as well as the contribution due to bulk deformation of the slab. It is important to note that for S-FAP nearly $55 \%$ of the combined stress and displacement contributions are compensated by the $\mathrm{dn} / \mathrm{dT}$ contribution. Fig. 89 gives the total phase distortion across the extracted aperture. where we can see the effects of pump roll-off in both directions as well as cooling effects in the vertical direction. Provided that each Mercury slab behaves similarly. we anticipate a total phase distortion of 6.7 waves $(+$ passes through $1+$ slabs). It is important to note. however. that the distortion given in Figs. 86 and 90 is low-order in structure. thus it is easily corrected by either a static corrector or a deformable mirror.
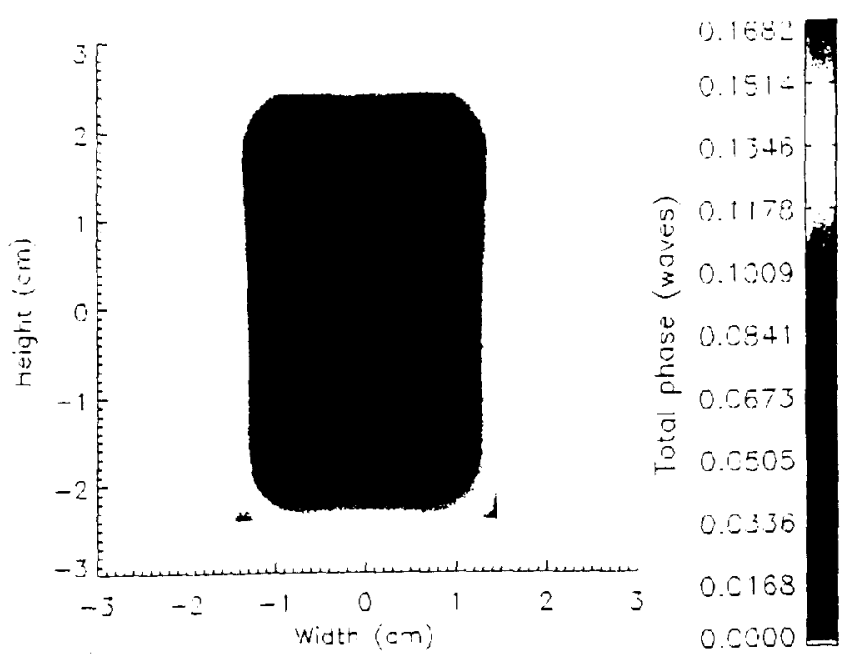

Fig 90. The total phase change across the extracted aperture. Note the slight wedge that is introduced in the height $(\mathrm{y})$ direction. This wedge is approximately $15 \%$ of the overall phase change. 


\section{Propagation Modeling with the Prop 92 Code}

PROP92: $=$ is used to perform numerical simulations of laser beam propagation in the Mercury Laser. PROP92 uses a Fourier technique to solve the nonlinear Schrödinger equation. yielding a representation of the single-polarization complex electric field $\mathrm{E}(\mathrm{x} . \mathrm{y} . \mathrm{z} . \mathrm{t})$ in two transverse directions (x.y) plus time (i.e., in 2D) as the beam transports through and is affected by a chain of optical elements. Signal gain and nonlinear-index effects are calculated in the near field. and propagation is done in the far field in

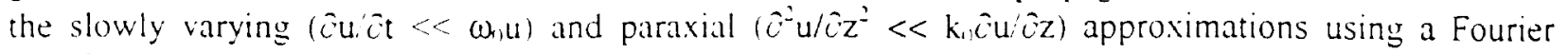
transform and its inverse according to

$u(\mathbf{r}, z+\delta z)=\frac{R}{R} \int \frac{d^{2} \kappa}{(2 \pi)^{2}} e^{i \frac{R_{11}}{R} \mathrm{k} \cdot \mathbf{r},-\frac{i k_{1}}{2 k_{1} R} \kappa^{-2} j z} \times \int d^{2} \mathbf{r}^{\prime} e^{-i \mathbf{k} \cdot \mathbf{r}^{\prime}} u\left(\mathbf{r}^{\prime}, z, t\right)$

Here the wave number $k_{11}=n_{11} \omega_{1} / c$ where $n_{1}$ is the index of refraction in the medium of propagation, $\omega_{n}$ is the laser's optical frequency. $c$ is the speed of light in vacuum. and $u(r, z, t)$ is the wave function remaining after the dominant plane-wave portion of the beam and its center-point position, tilt $K$. and curvature $R=$ $R(z+\delta z)$ have been removed from $E(x, y, z, t)$, where $R_{i}=R(z)$. Because $u$ is represented on a discrete mesh, the continuous transform above is actually calculated by a discrete fast Fourier transform (FFT).

Additional physics in PROP92 is implemented using various models. The safety margin against the collapse of intensity $I_{0}$ perturbations is tracked in nonlinear media to provide information on selftocusing (filamentation) based on the maximum transverse $\Delta \mathrm{B}$ integral exceeding a limit usually set at 2.3 radians $\left[\Delta \mathrm{B}=\left(2 \pi / \lambda_{\text {laser }}\right) \int\left(\mathrm{I}_{0} \gamma\right) \Delta z\right.$ for nonlinear index $\left.\gamma\right]$. A warning message is printed if the maximum projected self-focused beam intensity exceeds a user-entered breakdown intensity in any optic.

Damage from large optical fluence is based on a formula for Gaussian pulse shapes

$$
F_{D}=\alpha \tau^{\beta}
$$

and a phenomenological diffusion-like extension ${ }^{23}$ for non-Gaussian pulses. Here $F_{D}$ is the fluence at which damage sites first appear. $\tau$ is the Gaussian pulse width, and $\alpha$ and $\beta$ are material-dependent constants derived from the extensive experimental database of damage fluences for various materials for exposures with Gaussian pulses between $10 \mathrm{ps}$ and $100 \mathrm{~ns}^{2+}$ For each optic for which damage calculations are desired, PROP92 evaluates the extended damage integral ${ }^{25}$ for every spatial grid point in the calculational mesh. and issues a damage warning and the damaged area fraction if the integral exceeds the damage limit based on the inputted $F_{D}$. For Mercury, we chose the NIF-based values for $F_{D}$ given in Table 10. In Table 11, the other optical parameters.

Table 10. Optical damage fluences $F_{D}$ in $\mathrm{J} / \mathrm{cm}^{2}$

\begin{tabular}{|c|c|c|c|c|c|c|}
\hline & $\begin{array}{l}\text { AR } \\
\text { coatings }\end{array}$ & $\begin{array}{l}\mathrm{HR} \\
\text { coatings }\end{array}$ & $\begin{array}{l}\text { Yb:S-FAP } \\
\text { slabs }\end{array}$ & KDP & $\begin{array}{l}\mathrm{SiO}_{2} \\
\text { Polarizer in } \\
\text { trans. }\end{array}$ & $\begin{array}{l}\mathrm{SiO}_{2} \\
\text { Polarizer } \\
\text { in refltn. }\end{array}$ \\
\hline $\begin{array}{ll}1 \omega & (1047 \\
n m)\end{array}$ & $2+.6 \tau^{(1+4)}$ & $1+.9 \tau^{0.35}$ & $12.3 \tau^{0.35}$ & $24.2 \tau^{150}$ & $10.5 \tau^{0.35}$ & $12.4 \tau^{0.35}$ \\
\hline $\begin{array}{l}\text { Value } \quad \tau= \\
5 \text { ns }\end{array}$ & 46.8 & 26.2 & 21.6 & 54.1 & 18.4 & 21.8 \\
\hline
\end{tabular}


Table 11 Optical losses, refractive indices, and nonlinear coefficients.

\begin{tabular}{|c|c|c|c|c|c|c|c|}
\hline Item & Mirrors & $\begin{array}{l}\text { All } \\
\text { Windows }\end{array}$ & KDP & $\begin{array}{l}\text { Yb:S- } \\
\text { FAP } \\
\text { Slabs }\end{array}$ & Polarizer & $\begin{array}{l}\text { S.F. } \\
\text { Lenses }\end{array}$ & $\begin{array}{l}3 \omega \text { Focus } \\
\text { Lens }\end{array}$ \\
\hline $\begin{array}{l}\text { Single- } \\
\text { surface } \\
\text { transmissi } \\
\text { on } \\
\end{array}$ & 0.995 & 0.998 & 0.995 & 0.998 & $\begin{array}{l}.985 \text { trans } \\
.980 \text { retl. }\end{array}$ & 0.998 & 0.998 \\
\hline Bulk loss $/ \mathrm{cm}$ & & $6.3 \times 10^{-17}$ & $5.8 \times 10^{-2}$ & $5.0 \times 10^{-4}$ & $\begin{array}{l}\text { (Included } \\
\text { in above) }\end{array}$ & $6.3 \times 10^{-6}$ & $6.3 \times 10^{-6}$ \\
\hline $\begin{array}{l}\text { Retractive } \\
\text { indices } \\
(1 \omega)\end{array}$ & & $1 .+498$ & 1.50 & 1.620 & $1.4+98$ & $1.4+98$ & 1.4767 \\
\hline $\begin{array}{l}\text { Nonlin. } \\
\text { Coeff. } \\
\left(\mathrm{cm}^{2} / \mathrm{GW}\right)\end{array}$ & & $2.7 \times 10^{-7}$ & $2.7 \times 10^{-7}$ & $4.1 \times 10^{-7}$ & $2.7 \times 10^{-7}$ & $2.7 \times 10^{-7}$ & $3.55 \times 10^{-7}$ \\
\hline
\end{tabular}

We also included a 39-actuator deformable-mirror (DFM) model based on using a Hartmann sensor. The DFM was set to remove all optical phase aberrations acquired in two passes through the chain (i.e., in one round trip). Such a mirror is required to remove the crystal thermal aberrations because fixed corrector plates cannot adapt to the different thermal loads produced by varying the laser shot frequency.

\section{Optical wavefront distortions}

Our PROP92 modeling included a wavefront distortion (aberration) phase map for every optical surface in the laser. We obtained these phase maps by measuring the optical distortions for comparably sized components (i.e., for 7-cm NIF optics or few-cm Yb:S-FAP crystals) using a phase-stepping interferometer and a P-HOM technique. ${ }^{26}$ The P-HOM technique uses measurements of the reflected wavefront from both surfaces to determine the bulk phase properties of a sample from the transmitted wavefront. To obtain maps for more components than were measured. we calculated a Fourier transform of the measured map, took the complex square of the transform scaled by the length of the measurement to obtain what is called a power spectral density ${ }^{27}$ (PSD) in $\mathrm{nm}^{2} \cdot \mathrm{mm}$ for aberration spatial frequency $v$ in $\mathrm{mm}^{-1}$, took the square-root of this result multiplied by a random phase factor, and then calculated the inverse Fourier transform. The resultant phase maps contain the same spectral information, but randomized distortions. For ID maps. the 2D measurements were projected onto various angular orientations on the optic to obtain the worst effect.

We used the best grown small aperture Yb:S-FAP crystal to generate 14 randomized 2D phase maps. Due to the sub aperture crystals used. the phase maps are accurate up to spatial wavelengths of approximately one half the size of the Mercury gain slabs. In the modeling, we assumed that the DFM could remove the longer spatial wavelength effects not included in the PSDs. The shape of the PSD for the crystal is similar to that seen in many NIF optics which use the $v^{-1.55}$ power law scaling. We also obtained ID phase aberrations for each amplifier slab by sampling a pure power-law form, $\operatorname{PSD}(v)=\mathrm{A} \mathrm{v}^{-1.55}$. Such power-law PSDs are motivated by fractal considerations, because Church ${ }^{28}$ has shown that the aberration noise spectrum typical for highly finished optics is fractal in nature, and such a fractal spectrum has a power-law PSD. 


\section{D Modeling Results}

Our initial 2D calculations with $512 \times 512$ grid points showed that an input of $0.03 \mathrm{~mJ} / \mathrm{cm}^{2}(0.8$ $\mathrm{mJ}$ before shaping) would produce a total energy output of $91 \mathrm{~J}$. However. in our original baseline design we found that only $40 \%$ of the energy could be focused inside $5 x$ diffraction limited spot. In addition. several of the transport spatial filter optics were up to $21 \%$ over their damage thresholds. The damage problem arises primarily because the SF lenses are at non-relayed positions. Specifically, our calculations showed that the peak fluence increases up to $30 \mathrm{~J} / \mathrm{cm}^{2}$ for planes only $20 \mathrm{~cm}$ after the last Yb:S-FAP crystal. We have also found that the use of spatial-filter (SF) pintoles can greatly reduce the threat of optics damage to the lenses and mirrors. but with only marginal effect for the crystals (which are near relay planes). For example. if the transport SF magnification is set to 1.5 and the reverser SF magnification is set to unity, the use of one pinhole at a radius of 200 microradians at the entrance to the reverser lowers all lens and mirror peak fluences to values less than $60 \%$ of their damage thresholds for $5 \mathrm{~ns}$ pulses. Alternatively. if the pinhole radii on the four laser passes are set respectively to 200. 300 . 400 . and 500 microradians. the peak fluences are less than $50 \%$ of their damage thresholds even if the reverser magnification is lowered to its minimum value $(\sim 0.8)$ to allow placement of all components of the optical-switch isolator comfortably inside the reverser. In this case. the only consideration for damage is the reverser pickoff mirrors, which must be placed far enough (e.g.. $>+0 \mathrm{~cm}$ ) away from the SF focal point to avoid HR damage (or replaced with fused-silica prisms). The modeling then predicts entirely safe laser operation provided the pinholes can avoid significant damage while absorbing $\sim 1 \mathrm{~J}$ per shot $(\sim 1 \mathrm{~W}$ at $10 \mathrm{~Hz}$ ). The progress in our modeling effort can therefore be depicted as shown in Tables 12 a \& b below.

In summary we have found that with the new system layout (Fig 2) with the transport SF lens close to the relay plane. and the output transport SF magnification of 1.5 and the addition of a large pinhole at the entrance to the reverser then there is no threat of optical damage anywhere in the system and the final $1 \omega$ focus is $5 x \mathrm{DL}$ at $100 \mathrm{~J}$ output. The use of one pinhole appears to be sufficient, but other options are being investigated. such as adding large baffles in the first two passes to help with pencil beam and ghost suppression. We are encouraged by these results since these latter architectural changes permit the best and safest operation yet calculated for Mercury. 
Table 12a. Summary of the progress made in ID Modeling of the propagation of pulses in the Mercury (With 5-ns pulses unless otherwise stated)

\begin{tabular}{|c|c|c|}
\hline Design concerns & Design Change or Constraint & Outcome \\
\hline $\begin{array}{l}\Sigma B \geq 3 \text { for Sutton's thick crystal } \\
\text { windows }(1.125 \text { in) for } 1 \text {-ns } \\
\text { pulses and positive-frequency } \\
\text { PSD }=0.01 \mathrm{v}^{-1.55}\end{array}$ & $\begin{array}{l}\text { Use thinner windows and try to grow } \\
\text { crystals with this PSD (i.e.. } 2 \% \text { of NIF } \\
\text { "do-not-exceed" line) }\end{array}$ & $\begin{array}{l}\text { Moderation of } \\
\Sigma \mathrm{B} \text { at I ns to } \\
\text { minimize } \\
\text { nonlinear } \\
\text { growth }\end{array}$ \\
\hline $\begin{array}{l}\text { Many optical components are } \\
\text { damaging for estimated "reill" } \\
\text { crystal quality; cannot focus } \\
\text { much of the } 100 \mathrm{~J} \text { output through } \\
5 \times \text { DL hole }\end{array}$ & $\begin{array}{l}\text { Crystals positive-firequency PSD must } \\
\text { not exceed unity: } v^{-1.55} \text { (i.e.. } \mathrm{rms}^{-1} \\
\text { surface roughness } 0.06 \text { to }+1 \mathrm{~mm}^{-1}< \\
\text { \& nm per optical surface) }\end{array}$ & $\begin{array}{l}\text { Effective focus } \\
\text { inside }+X \mathrm{DL} \\
\text { square hole is } \\
\text { possible }\end{array}$ \\
\hline $\begin{array}{l}\text { Reverser SF mag }=0.4 \text { disallows } \\
\text { enough space for } P C / \text { Polzr.. and } \\
\text { causes pickoff mirrors to operate } \\
\text { at } 3 X \text { to } 4 X \text { and final transport } \\
\text { SF lens at } 2 X \text { respective damage } \\
\text { thresholds }\end{array}$ & $\begin{array}{l}\text { Change reverser SF mag from } 0.4 \text { to } \geq \\
0.8 \text { to have sufficient room for } \\
\text { components inside reverser, and to } \\
\text { decrease optical damage threat } \\
\text { beyond the reverser }\end{array}$ & $\begin{array}{l}\text { 1D operation at } \\
\leq 90 \% \text { of } \\
\text { damage: focus } \\
\text { of only } \sim 1 / 3 \text { of } \\
\text { maximum beam } \\
\text { energy through } \\
5 \times \text { DL hole }\end{array}$ \\
\hline $\begin{array}{l}\text { Can focus only about } 1 / 3 \text { of } 100-\mathrm{J} \\
\text { beam energy through } 5 X \mathrm{DL} \text {. } \\
\text { which is } 3 X \text { less than Mercury } \\
\text { goal }\end{array}$ & $\begin{array}{l}\text { Improve crystal quality so PSD is } 10 \% \\
\text { of that for Bruce Chai crystals (i.e., } \\
\text { positive-frequency PSD }<0.1 \mathrm{v}^{-1.55} \text { ) }\end{array}$ & $\begin{array}{l}\text { Can focus } 100 \mathrm{~J} \\
\text { through } 5 \times \mathrm{DL} \\
\text { hole using } 10^{4} \text { - } \\
\text { urad-radius } \\
\text { pinholes }\end{array}$ \\
\hline
\end{tabular}


Table I2b Summary of the progress made in 2D Modeling of the propagation of 5-ns.

\begin{tabular}{|c|c|c|c|}
\hline Design concerns & $\begin{array}{c}\text { Design Change or } \\
\text { Constraint }\end{array}$ & Outcome & Comments \\
\hline $\begin{array}{l}\text { All tinal optics (MS+ } \\
\text { transport SF lenses) are } \\
\sim 1.2 \times \text { damage threshold. } \\
\text { and can focus only } 40 \% \\
\text { of the maximum beam } \\
\text { energy through } 5 \times \mathrm{DL} \\
\text { hole }\end{array}$ & $\begin{array}{l}\text { - Reduce "as-grown" } \\
\text { crystal PSD by } \\
\text { tactor of } 70 \% \text {. } \\
\text { and/or } \\
\text { - Change transport SF } \\
\text { mag from } 1.0 \text { to } 2.0\end{array}$ & $\begin{array}{l}\text { No optic is } \\
\text { above damage. } \\
\text { but can focus } \\
\text { only }+7 \% \text { of } \\
\text { beam energy } \\
\text { - No damage } \\
\text { and can focus } \\
2 / 3 \text { of beam } \\
\text { energy }\end{array}$ & $\begin{array}{l}\text { Worst damage occurs for } \\
\text { optics placed at non- } \\
\text { relayed positions. so if } \\
\text { possible. one SF lens } \\
\text { should be located as } \\
\text { close as possible to the } \\
\text { crystals }\end{array}$ \\
\hline $\begin{array}{l}\text { Many optics are still } \\
\text { operating at } 80 \text { to } 90 \% \text { of } \\
\text { damage threshold. and } \\
\text { can focus only } 2 / 3 \text { of } \\
\text { maximum beam energy } \\
\text { through } 5 \times \text { DL hole }\end{array}$ & $\begin{array}{l}\text { Set transport SF mag = } \\
1.5 \text { to get equipment in } \\
\text { lab and move first } \\
\text { transport SF lens close } \\
\text { to crystals and use } \\
\text { pinholes in SFs: } \\
\text { - Radii } 200.300 .400 . \\
\text { and } 500 \text { on laser } \\
\text { passes } 1.2 .3 . \text { and }+ \\
\text { (tailored because } \\
\text { later passes have } \\
\text { greater energy } \\
\text { depositions for same } \\
\text { pinhole radius) }\end{array}$ & $\begin{array}{l}\text { 90) to } 93 \% \text { of beam } \\
\text { energy can be } \\
\text { focused inside } \\
5 \times \text { DL hole. } \\
\text { AND } \\
\text { - All fluences < } \\
50 \% \text { of } \\
\text { damage } \\
\text { thresholds } \\
\text { (plus better } \\
\text { ghost } \\
\text { rejection) }\end{array}$ & $\begin{array}{l}\text { Reverser pickoff mirrors } \\
\text { must be placed }>40 \mathrm{~cm} \\
\text { from SF focal plane to } \\
\text { avoid damage. } \\
\text { Pinhole deposition at } 10 \\
\mathrm{~Hz} \text { is } \geq 1 \mathrm{~W} \text {, which } \\
\text { requires some form of } \\
\text { cooling AND further } \\
\text { investigation regarding } \\
\text { pinhole closure. } \\
\text { Pinholes }>500 \mu \mathrm{rad} \text { are } \\
\text { essentially full open }\end{array}$ \\
\hline
\end{tabular}




\section{Integration Experiments}

\section{A. Gain Measurements with Surrogate Nd:Glass Amplifier Media}

The small signal gain of the gas cooled amplifier was measured with seven Nd:phosphate glass slabs mounted in the metal vanes. The glass slabs will serve as surrogate gain media until the crystal slabs are ready and will allow us to test many aspects of the pump delivery and gas cooled amplifier hardware. The phosphate LHG-8 slabs have a small amount of absorption at $900 \mathrm{~nm}$ within the wings of the absorption spectra $\left(\sim 10^{-21} \mathrm{~cm}^{2}\right)$. This allows us to perform gain measurements with the same set of diodes that have been optimized for Yb:S-FAP. The slabs were pumped with $30 \mathrm{~kW}$ of diode light with $750 \mu s$ pulses at $3 \mathrm{~Hz}$ repetition rates. The 40-bar diode tiles were mounted in one quadrant of the backplane. which accounts for the asymmetric tluorescence protiles shown below.

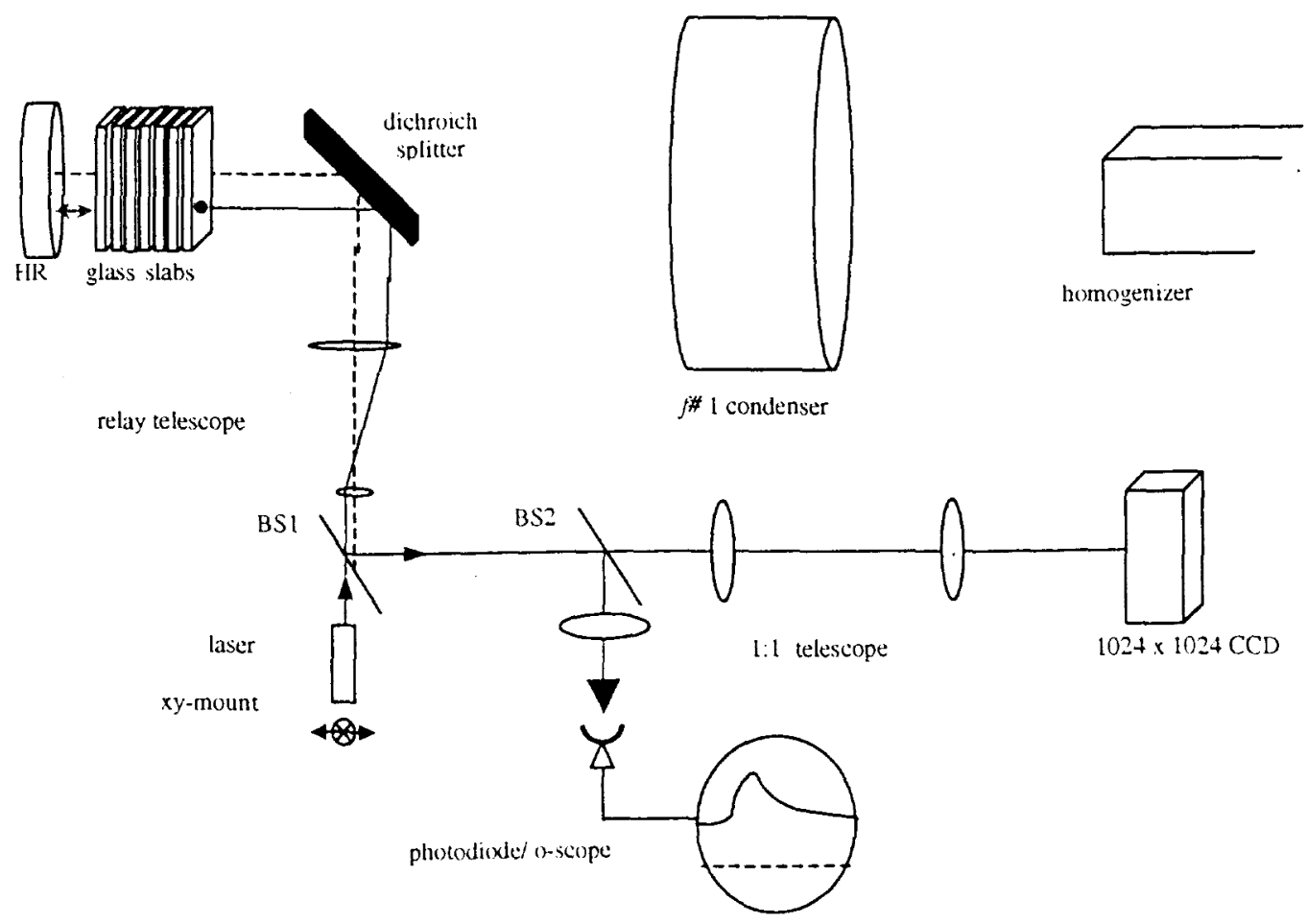

Fig. 91 Schematic diagram of the experimental setup.

Fig. 91 shows a schematic of the experimental setup. The pump light emerging from the homogenizer was imaged onto the first slab with $\mathrm{f} / \mathrm{l}$ condenser optics. The diode array was cooled to -23.5 ${ }^{\prime} \mathrm{C}$ in order to shift the output wavelength to $887.9 \mathrm{~nm}$, which is close to the $880 \mathrm{~nm}$ absorption peak in LHG-8 glass. Only $2 \%$ of the pump light incident on the amplifier head was not absorbed and leaked out the back. An image of the fluorescence was obtained and used to map the pump light distribution. The spatial gain distribution has a direct correlation to the fluorescence distribution since both are related to the upper laser level number density. The fluorescence distribution was used to assess the optimum locations of 
the small-diameter cw $1.053 \mu \mathrm{m}$ probe beam. Fig. 92 shows an image of the fluorescence and the 9 locations where the gain was measured.

The cw probe beam was double passed through the gain media and focused into a photodiode connected to an oscilloscope. The voltage signal recorded is directly proportional to the gain. As can be seen in Fig. 93. the gain signal increases during the $750 \mu \mathrm{sec}$ diode pulse length as the population inversion builds and reaches a peak value at the end of the pump pulse. The signal then decays back to its original DC level. During the measurements. a small amount of fluorescence leaked into the photodiode. This background signal was accounted for by measuring the signal level on the oscilloscope with the diode light on and probe laser oft.

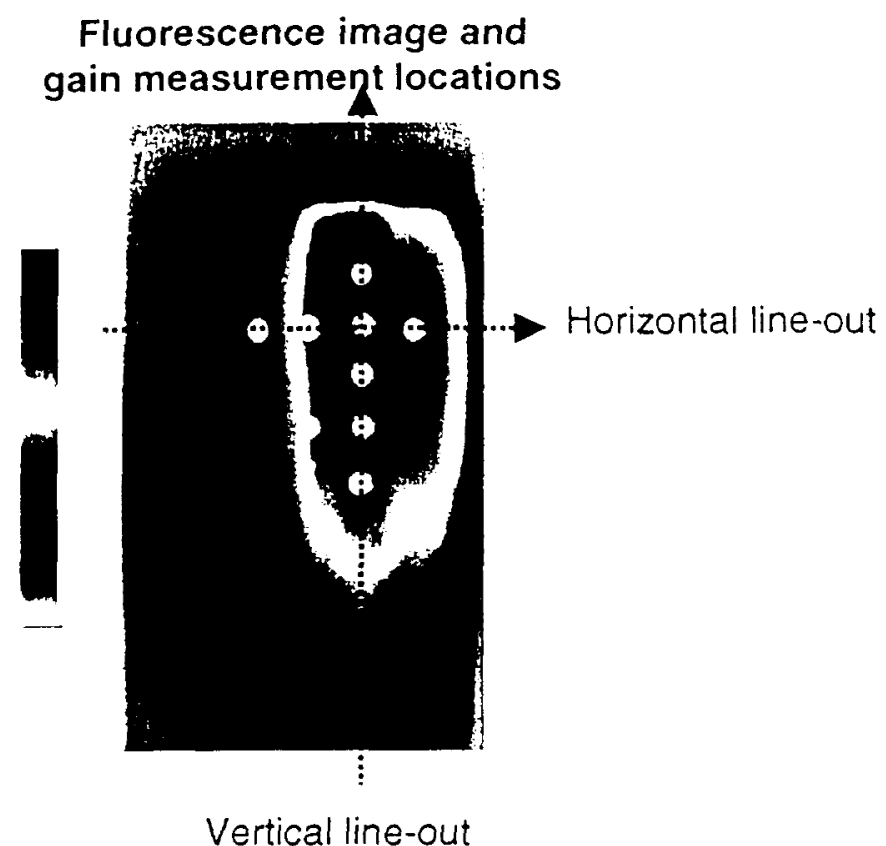

Fig. 92 Camera image of the time-integrated 2-D fluorescence profile from all 7 slabs. The white bullets mark the locations where the gain was probed. The dotted lines show the locations for the fluorescence line-outs in Fig. 94.

\section{Analysis}

The double passed probe beam experiences an absolute exponential gain given by:

$$
I(t)=I_{0} \exp \{2 N(t) \sigma L\}
$$

where $I_{v}$ is the incident probe intensity (well below the medium's saturation intensity), $N(t)$ is the upper laser level population, $\sigma$ is the stimulated emission cross section and $\mathrm{L}$ the gain length. The amplifier double-pass gain can be extracted from the oscilloscope data by dividing the waveform by the average 
DC-level immediately preceding the pump-pulse. The upper level population is governed by the differential equation:

$$
\frac{d}{d t} N(t)=S(t)-\frac{N(t)}{\tau}
$$

where $t$ is time. $S(c)$ is the volumetric source rate and $\tau$ is the upper level lifetime. The pump power $P_{\text {, }}$ incident on the amplifier window was measured with a glass calorimeter. To determine the source rate $S(t)$ we summed over the discretized fluorescence shape in Fig. 94 and then normalized the sum to the incident pump power. Because the fluorescence is observed from the same end we were pumping. the data is naturally integrated over the absorption depth $L$. The total incident power is then related to the localized pump intensity $P_{1 . j}$ at pixel i. j by:

$$
P_{0}=\delta x \cdot \delta y \cdot \sum_{j} \sum_{i} P_{i}, i
$$

where $\delta x, \delta y$ are the dimensions of aperture the CCD pixels imaged. The source rate per unit volume averaged over the absorption depth $L$ at pixel $(i, j)$ is:

$$
S(t)_{(i, j)}:=\frac{P_{i, j}}{h_{V} \cdot L} \cdot \Phi(t)
$$

where hv is the pump photon energy. $\Phi(t)$ is a step function that turns the source off after a time lapse matching the diode pump pulse length. The solution to equation (2) for any region of the aperture represented by pixel $(i, j)$ is given by:

$$
N(t)_{(i, j)}=e^{-\frac{t}{\tau}} \cdot \int_{0}^{t} \varepsilon \cdot S\left(t^{\prime}\right)_{(i, j)} \cdot e^{\frac{t^{\prime}}{\tau}} d t^{\prime}
$$

where $\varepsilon$ is the transport efficiency accounting for reflection and absorption losses at the cell window and slab. We estimate this value to be approximately 0.77 (a total of 16 surfaces and $5.25 \mathrm{~cm}$ worth of laser glass with a passive loss $~ 0.001 \mathrm{~cm}^{-1}$ ). The next step involves fitting the peak intensity of Eqn. 1 (using eqns. $2-5$ and the fluorescence profile) to the peak experimental $\mathrm{cw}$ probe data point, where the only adjustable parameter used in fitting the data was $\varepsilon$. Other parameters used in the model such as lifetime and cross section were found in the literature. The best fit the data yielded a value of $\varepsilon=0.76$. The fluorescence data was then normalized to the peak gain value and lineouts were compared with the cw probe data (Fig. 94). We found the data and model to be in good agreement. In the future, full beam gain measurements will be made to compare against our 2-D propagation codes. 


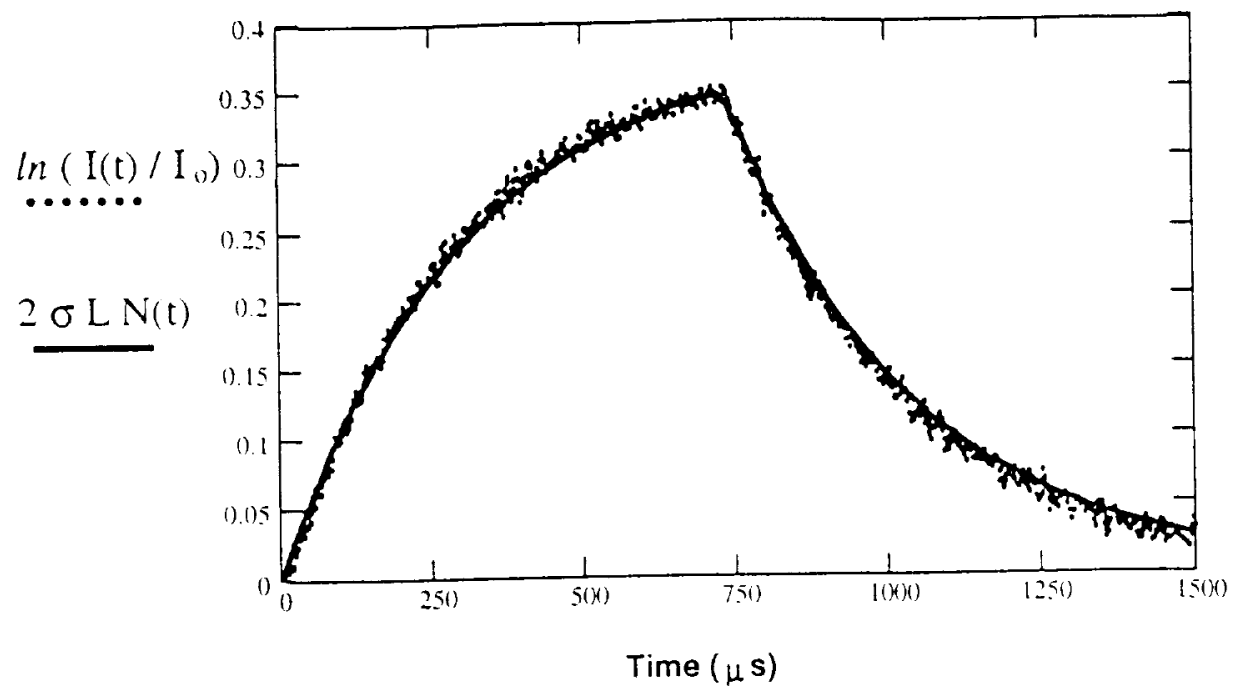

Fig. 93 The experimental trace of the intensity on the photodiode as a function of time. Note the peak of the gain curve occurs at the end of the $750 \mu \mathrm{sec}$ pump pulse.
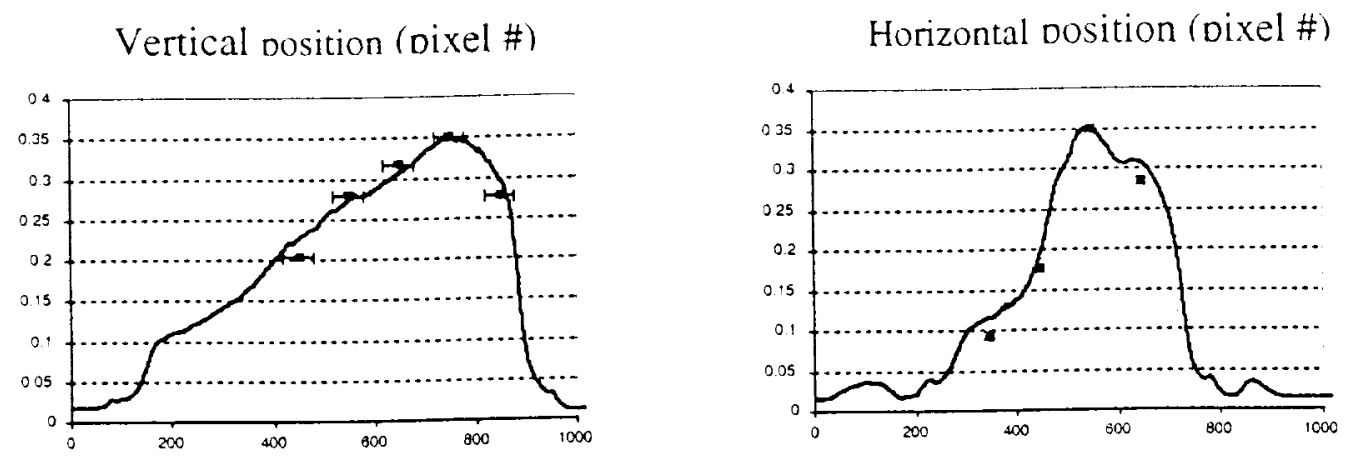

Fig. 94 The fluorescence shape was calibrated by our point gain measurement at the peak location of the tluorescence. The vertical and horizontal line-outs follow the $\mathrm{cw}$ point gain measurements. 


\section{B. Measurements of Wavefront Distortions}

An optical diagnostic system for measuring full aperture wavefront distributions has been recently activated in the Mercury laboratory. The system is based on the previously built Large Aperture Diagnostic System (LADS) developed and thoroughly tested in measuring the optical performance of the NIF prototype amplifiers in AMPLAB ${ }^{21}$. Fig 95 a shows the basic components of the wavefront measurement setup. A representative set of tringe data is shown in Fig 95 b.

(a)

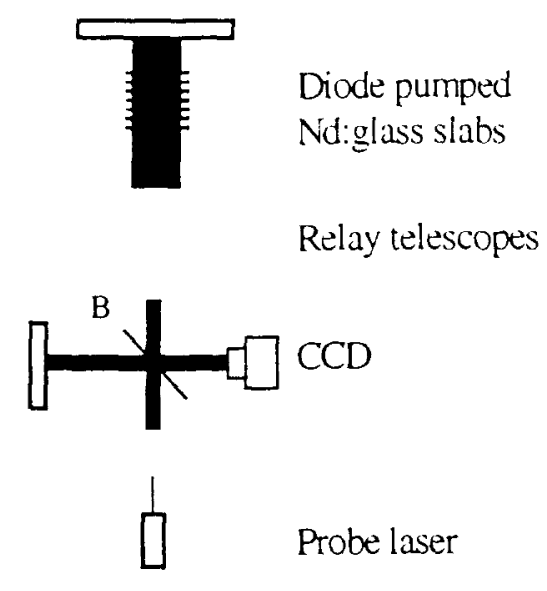

( b )

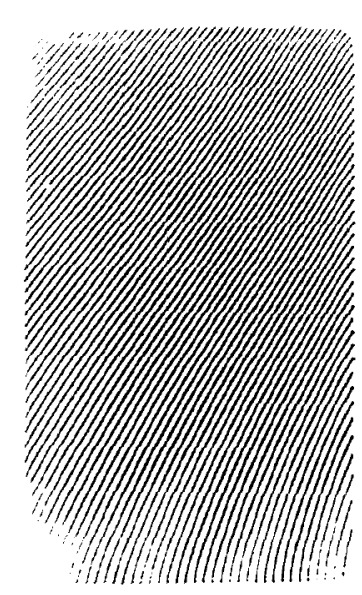

Fig. 95 (a) The basic Twyman-Green setup for measuring wavefront distortions in the Mercury Amplifier. (b) Typical fringe data. The window to the amplifier cell is the limiting aperture.

A reference beam (with the appropriate tilt) is split-off at the beam-splitter BS and re-combined with a probe beam. The interference fringes are captured with a $1024 \times 1024$ pixel CCD camera. The software program stores and analyzes the background wavefront using a Fourier transform based algorithm. The phase retrieval method ${ }^{30}$ filters the induced carrier frequency (or tilt) while retaining the spatial frequencies that cause the fringe modulations. The wavefront tilt translates the centroid of the carrier-frequency modulation away from the origin as can be seen in Fig 96. The software analysis first removes the on-axis frequencies (due to intensity variations across the aperture) and then translates the centroid back to the origin before the inverse Fourier transform calculation is performed. Note that the frequency spread observed around the centroid is due to the fact that the probe beam is not a flat wavefront. If the wavefront were flat. then the centroid would be a delta function.

The inverse Fourier transform of the modified data thus yields the corrected wavefront except for phase jumps in increments of one wave. The software corrects for the phase jumps by first assessing the sign of the slope where the phase jump occurs. It then reconstructs the phase profile by adding or subtracting a wave in order to make the phase front continuous. 


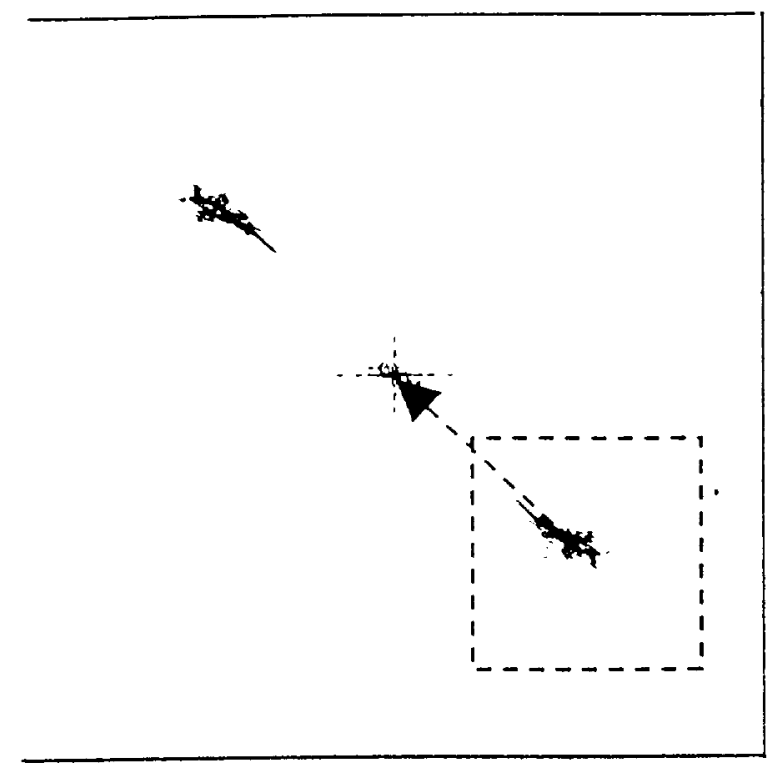

Fig. 96 The Fourier transform of the tringe data. The spread around the carrier frequency (or tilt) contains the frequency components of the wavefront. The carrier frequency is removed by shifting the centroid to the origin prior to transtorming back to the spatial domain.

The interferometer setup was recently activated and calibrated and was found to have a system resolution of $\lambda / 100$. The initial experiments to characterize the gas cooled head included:

1) The wavefront distortion due to mounting a single slab into a vane element.

2) The transmitted wavefront distortions of the fully assembled amplifier ( 7 glass slabs) under static conditions (no pressure, no flow).

3) The transmitted wavefront distortions of the assembled amplifier under pressurized (4 atmospheres) but otherwise static conditions.

4) The transmitted wavefront distortions of the assembled amplifier under the maximum operating helium gas cooling flow of $100 \mathrm{~m} / \mathrm{s}$.

5) The transmitted wavefront distortions of the amplifier under conditions of full flow, with one quadrant diode pumped at up to $3 \mathrm{~Hz}, 100 \mathrm{amp}, 750 \mu$ s pulses.

\section{Detailed Experimental set-up}

Fig. 97 shows the experimental layout of the Mercury LADS. A $150 \mathrm{~mW}$ diode-pumped single frequency cw YLF laser is expanded with three telescopes to fill the $38 \mathrm{~mm}$ by $58 \mathrm{~mm}$ window to the amplifier head containing seven $42 \mathrm{~mm} \times 62 \mathrm{~mm}$ LHG-8 glass slabs. An uncoated beam splitter before telescope T2 provides a reference beam and acts as a beam combiner for the probe and reference beams. The beams interfere and produce fringes that can be adjusted in tilt and density by adjusting the reference flat, which is also at a relay plane. The fringe-dense image is relayed to the scientific CCD by the $1: 1$ telescope $\mathrm{T} 3$. The slabs are approximately $150 \mathrm{~mm}$ from the back mirror relay plane thus mitigating the effects of edge diffraction at the CCD image plane.

The $\mathrm{Cw}$ probe beam was chopped to filter out vibrations and exposed the CCD for approximately $750 \mu \mathrm{s}$. The Hepa-filters mounted on the ceiling for cleanliness caused air turbulence and therefore beam tubes were added in order to shield the beam path. This improved the wavefront measurement RMS to 
within $0.02 \lambda$. The best wavefront RMS $( \pm 0.005 \lambda)$ was obtained by turning off the Hepa filters and averaging 16 separate wavefront images.

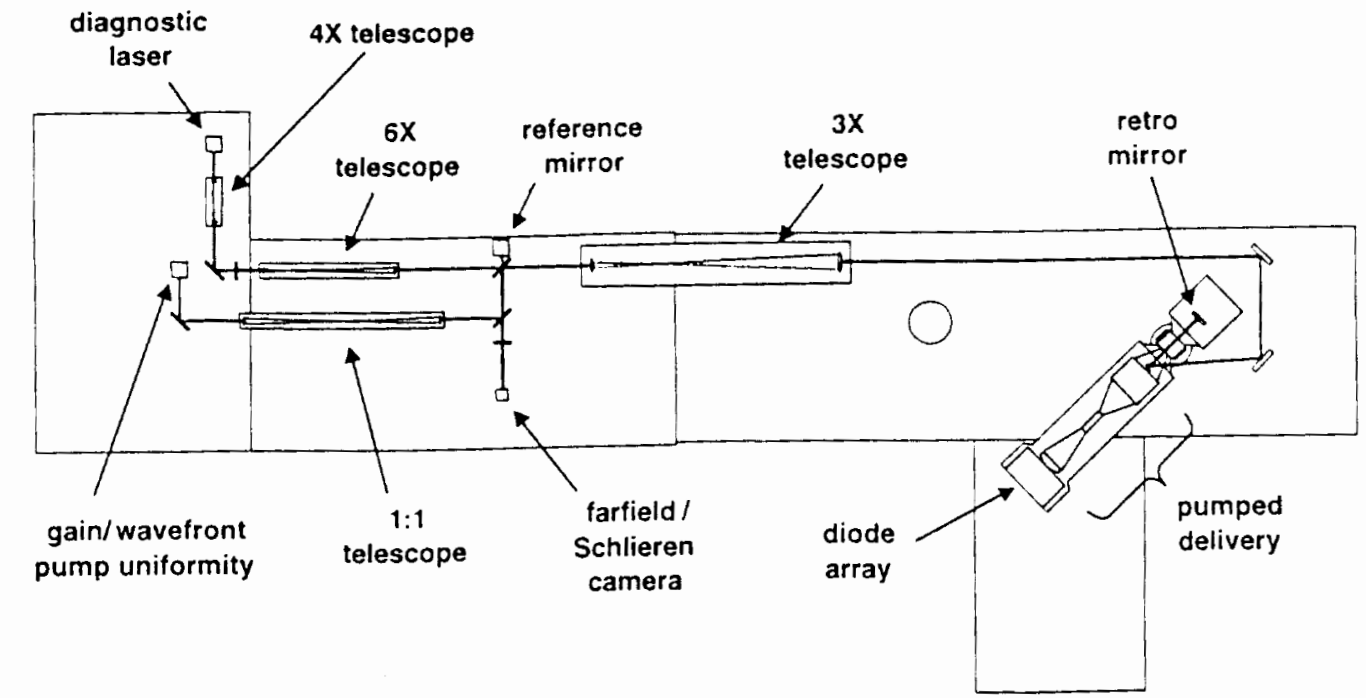

Fig. 97 Wavefront and gain LADS experimental setup.

\section{Slab mounting (Experiment 1)}

The seven amplifier slabs are individually mounted into aluminum vanes with a compliant uropol elastomer. The wavefront distortion of a single vane-mounted slab was measured (Fig. 98) and found to have a single pass wavefront error of $<0.1 \lambda$ peak-to-valley.

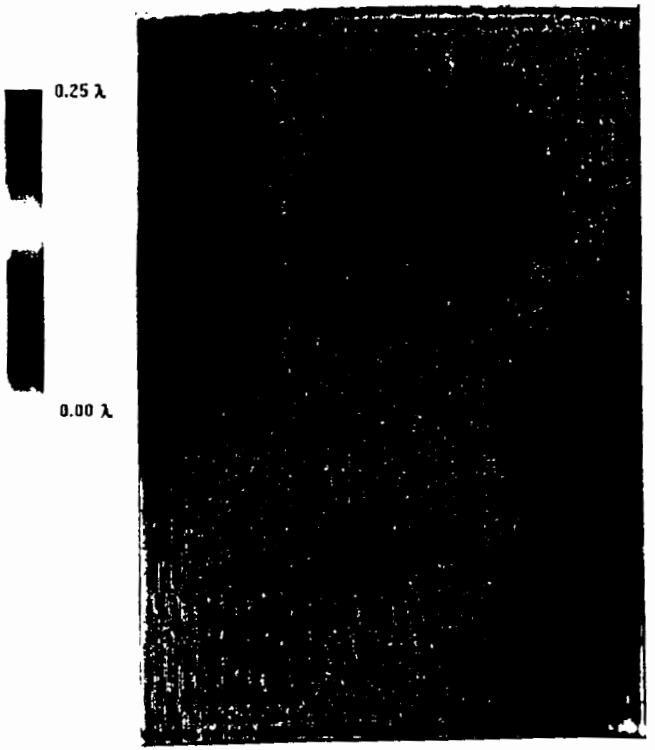

Fig. 98 Residual wavefront distortion for a single slab mounted in a flow vane. 
This data agreed with independent data collected with the same unmounted slab using a Zygo interferometer. The high frequency ripples appearing at the bottom of the wavefront-map have an amplitude of about $0.0 ! \lambda$ peak-to-valley. The source of these modulations is uncertain and we speculate that they could be due to the polishing (sleek marks) or may stem from etalon effects caused by the ARcoating reflectivity and the parallel slab arrangement.

\section{Assembled amplifier wavefront error under static, pressurized and gas-flow conditions}

\section{(Experiments 2-4)}

The wavefront error for a fully assembled amplifier head with 7 vane mounted glass slabs was measured for a variety of conditions. The static residual wavefront error of the system was measured by averaging a set of 16 wavefronts resulting from interferograms taken one minute apart. The static wavefront revealed low spatial frequencies of the order of $1 / 16 \lambda$ peak-to-valley and is shown in Fig. $99 \mathrm{a}$. The system was then pressurized to 3 atmospheres (gauge) in order to isolate the effect of bulging windows on the transmitted wavetront. Once again 16 interferograms were collected. analyzed and the resulting wavetronts averaged. The static wavefront (Fig. 99a) was then subtracted. The resulting wavefront contains only the effect of the pressurized cell and can be seen in Fig. 99b. Finally. the effect on the wavetront of the helium coolant flow was derived in the same manner. The result is shown in Fig. $99 \mathrm{c}$. More detailed measurements will be needed to fully characterize the system under diode pumping and with S-FAP crystals. However. the wavefront analysis performed so far with surrogate glass slabs show that there are no significant wavefront errors due to pointing or gas flow in the amplifier head. In addition. the single-pass wavefront error of $\sim 1 / 20 \lambda$ per pass is easily correctable with today's deformable mirror technology.

( a )

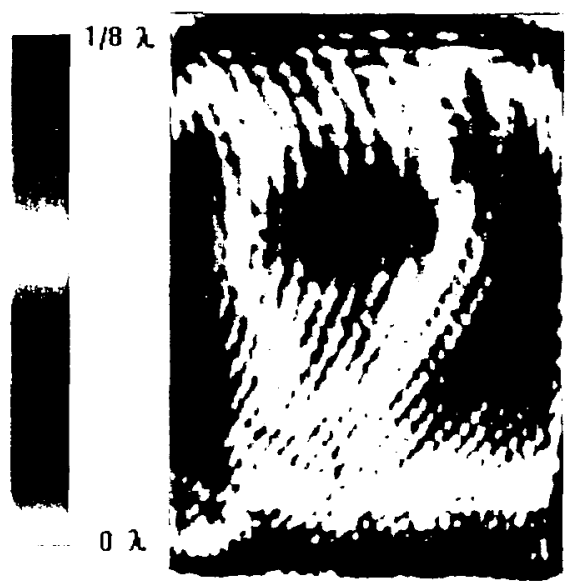

(b)

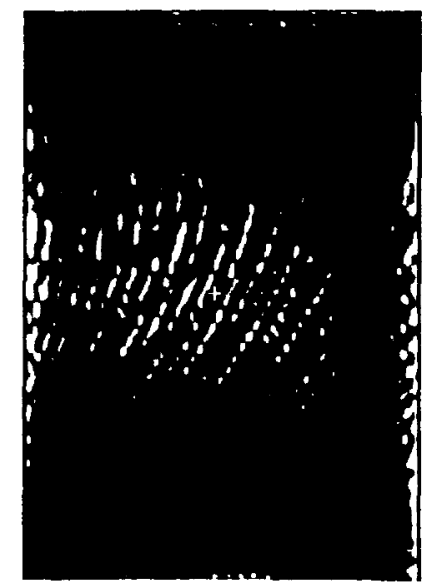

(c)

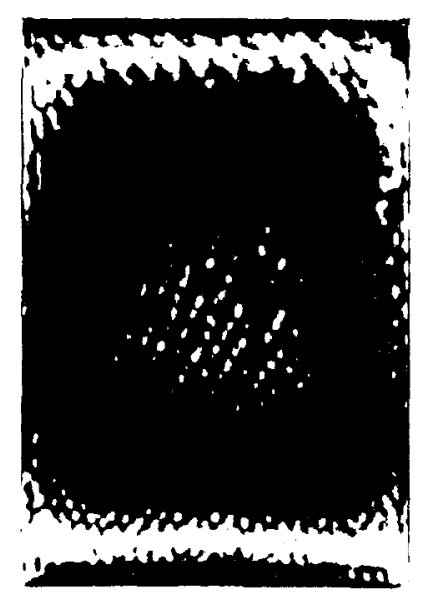

Fig. 99 (a) Single pass wavefront error for the static amplitier. (b) Differential single pass wavefront error for the pressurized but static amplifier (the static wavefront in (a) has been subtracted). (c) Differential single pass wavefront error for the pressurized amplifier with full helium flow. 

(Experiment 5)

The pump diode array consists of 400 diode bars populating one quadrant of the back plane. The wavefront error was measured while optical pumping in the first end-to-end use of the amplifier hardware. controls and diagnostics. At the maximum pumping used for the glass surrogate slabs, the diode stack parameters were set at $3 \mathrm{~Hz} .750 \mu \mathrm{s} .100 \mathrm{amps}(40 \mathrm{~kW}$ optical) and the helium coolant flowed at $100 \mathrm{~m} / \mathrm{s}$. The diodes were cooled to -30 " $\mathrm{C}$ to shift the diode array wavelength towards the peak of the $880 \mathrm{~nm} \mathrm{Nd}$ :glass absorption peak. The absorption at $888 \mathrm{~nm}$ was measured off line to be $60 \%$ per slab. Under these conditions the peak-to-valley wavetront error obtained (Fig. 100) inside the intended extraction aperture $(3 \mathrm{~cm} \times 5 \mathrm{~cm})$ is about 0.16 waves/slab/pass. The data will be used to validate our numerical design codes.

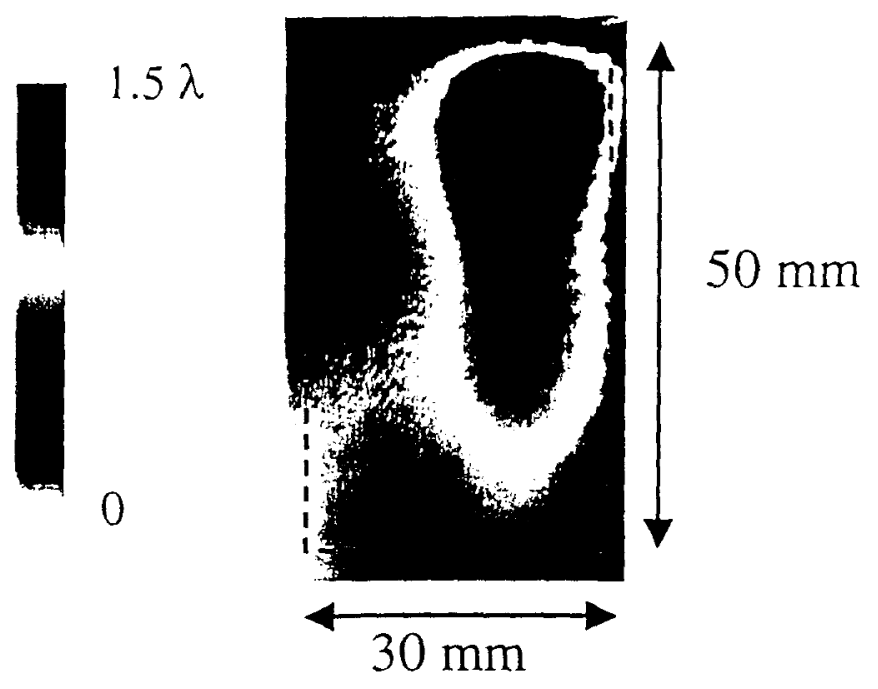

Fig. 100 Single pass wavefront error for the seven-slab fully assembled, gas-cooled, amplifier. Only one quadrant was pumped with 400 diode bars operating at $3 \mathrm{~Hz}, 750 \mathrm{~ms}, 100$ amperes. The extraction beam foot-print is depicted by the box within. 


\section{Measurements of the temperature distribution in the lens duct and homogenizer}

The advanced architecture design. discussed in Section 4B. requires that the extraction beam pass through the middle of the pump delivery components (homogenizer and duct). These components will experience a temperature rise due to the small amount of pump light absorbed and this will cause convective currents to occur within the homogenizer and duct. As a result, the extraction beam wavefront will become distorted when passing from one amplifier system to the next. A solution to this issue is to cool the duct and homogenizer and place them under vacuum. In order to ascertain the cooling capacity required an experiment was performed to measure the temperature rise and extrapolate the steady state conditions.

The Mercury diode array $(1 / 4$ filled $)$ was operated at an average power output of 90 watts for several hours. Thermocouples were attached to the outside of the duct and homogenizer to measure the temperature rise as a function of time. A lumped heat capacity model was used to derive the cooling rate from the logarithmic slope of the bulk temperature decay after the diodes were tumed-off. An extrapolation to the steady-state temperature was used to find the power dissipated from the homogenizer and duct surfaces resulting from the small amount of pump light absorbed. It was determined that the duct and homogenizer absorbed $0.6 \%$ and $3.5 \%$ respectively of the diode pump light.

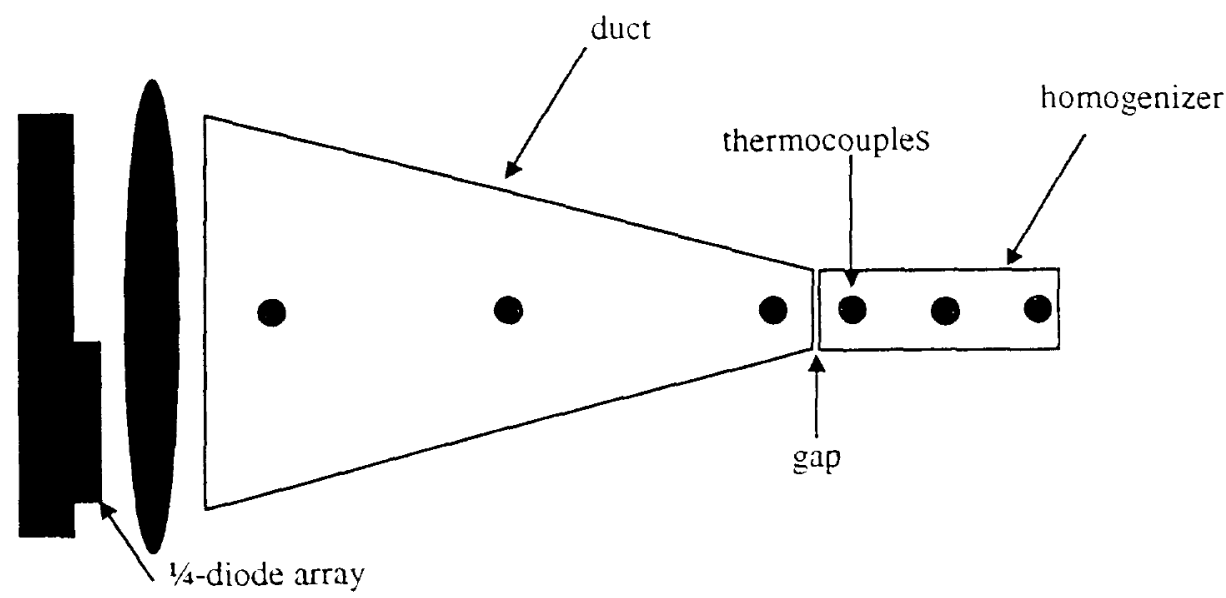

Fig. 10l. Schematic of the experiment. The diode-array was operated at a peak power output of $40 \mathrm{~kW}$ with a $0.75 \mathrm{~ms}$ pulse-width at a $3 \mathrm{~Hz}$ repetition rate. Thermocouples were mounted on all four sides of the duct and homogenizer.

The 1/4-diode-array was turned on at 100 amps generating 90 watts of optical average power. The diode light was transported by the duct and homogenizer and into a beam dump at the output. There was $1 / 2-\mathrm{mm}$ gap between the duct and homogenizer. Twenty-four thermocouples monitored the temperature at various locations (Fig. 101). The temperatures measured by the homogenizer thermocouples were similar within $\pm 0.2^{\circ} \mathrm{C}$. The temperatures measured on the duct were higher but also agreed within $\pm 0.2^{\circ} \mathrm{C}$. 


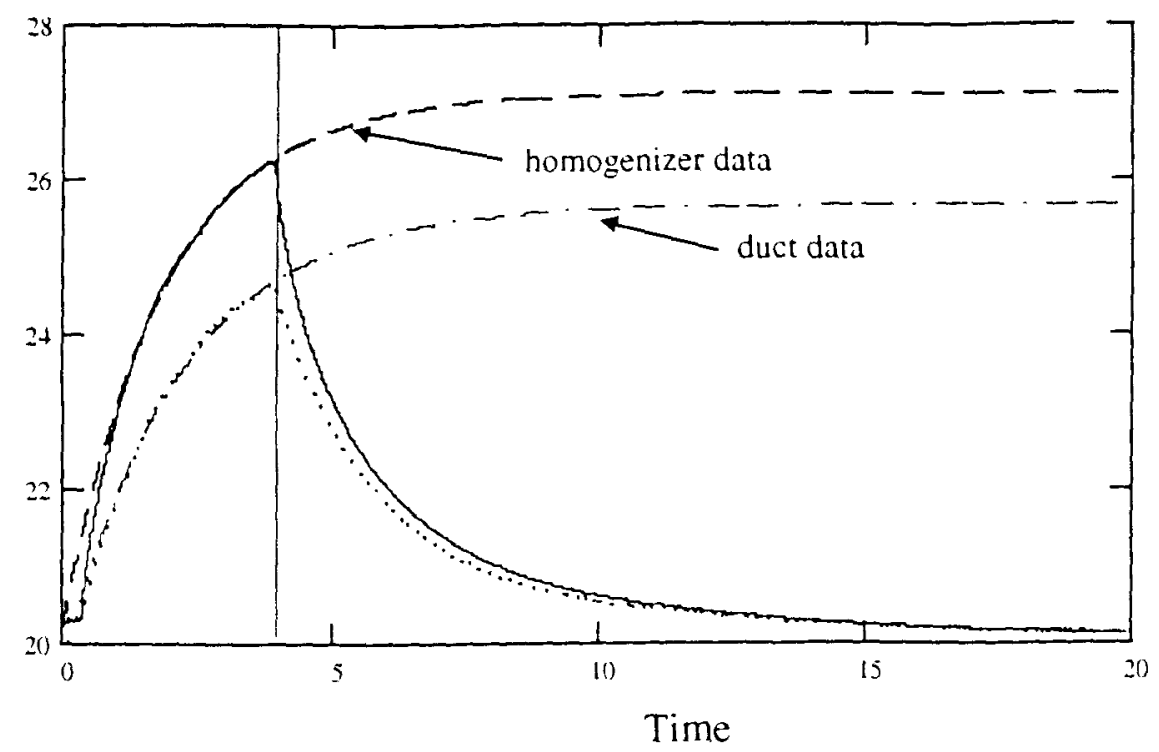

Fig. 102 The temperature excursion for the homogenizer and duct bulk temperatures is graphed. The data were fitted by a simple exponential rise function to find the steady state temperatures that would have been reached had the diodes been left on indefinitely. The dashed line indicates the start of the cooling after diodes were turned off.

\section{Lumped heat capacity model}

If the internal resistance to heat transfer within an aluminum body is assumed negligible in comparison with the external convective resistance (as in the present case), then a lumped-heat-capacity analysis can be used. Initially, the diode light that is absorbed by the walls produces a heat input $q$. The heat input is balanced by an increase in the internal energy of the body and the heat loss due to free convection into the environment and can be described by:

$$
\mathrm{q}=\mathrm{h} \mathrm{A}\left(\mathrm{T}-\mathrm{T}_{e}\right)-\rho \mathrm{C} \cdot \mathrm{V}(\mathrm{dT} / \mathrm{d} t)
$$

where $h$ is the natural convection coefficient, $A$ is the heat transfer area, $T$ is the bulk temperature, $T$, is the environment (sink) temperature, $C$ is the specific heat, $\rho$ is the density of the material, and $t$ is time. All the parameters are easily obtained except for $q$ and $h$. We tirst determine $h$ by solving the above equation for when $q=0$ (after the diodes are turned off) with the initial condition that $T=T_{0}$ at $t=0$. We find that equation (1) has the following solution:

$$
\operatorname{Ln}\left\{\left(\mathrm{T}(t)-\mathrm{T}_{e}\right) /\left(\mathrm{T}_{0}-\mathrm{T}_{e}\right)\right\}=-(\mathrm{h} \mathrm{A} / \rho \mathrm{CV}) t
$$

A straight line is fit to the data to obtain the slope, which equals the product $h \mathrm{~A}$.

To determine $q$, equation 1 is solved in the steady state regime where $d T / d t=0$. The heat flowing into the aluminum body can be obtained from knowing the steady state temperature and $\mathrm{h} \mathrm{A}$. The steady state temperature $\left(T_{s s}\right)$ was obtained by fitting a simple exponential rise (Eqn 3 ) to the data (see Fig. 102).

$$
\mathrm{q}=\mathrm{hA}\left(\mathrm{T}_{\mathrm{sis}}-\mathrm{T}_{\mathrm{s}}\right) \quad \text { where } \mathrm{T}(\mathrm{t})=\mathrm{T}_{\mathrm{sis}}(\mathrm{l}-\exp (-\lambda \mathrm{t}))
$$

\begin{tabular}{|c|c|c|c|c|c|c|}
\hline & $\begin{array}{l}\text { Slope } \\
\left(\sec ^{-1}\right)\end{array}$ & $\begin{array}{l}\mathrm{hA} \\
\left(\mathrm{W} /{ }^{\circ} \mathrm{K}\right)\end{array}$ & $\begin{array}{l}\text { Mass } \\
(\mathrm{kg})\end{array}$ & $\begin{array}{l}\text { Heat } \\
\text { (watts) }\end{array}$ & $\begin{array}{l}\Delta \mathrm{T} \\
\left({ }^{\circ} \mathrm{K}\right)\end{array}$ & $\begin{array}{l}\text { Fraction } \\
\text { absorbed }\end{array}$ \\
\hline Duct & -0.465 & 0.10 & 3.32 & 0.554 & 5.54 & $0.6 \%$ \\
\hline Homogenizer & -0.543 & 0.45 & 0.86 & 3.15 & 6.98 & $3.5 \%$ \\
\hline
\end{tabular}

The table below summarizes the results;

The fraction of heat absorbed absorbed indicates that there is 6 times more energy deposited in the homogenizer than in the duct. This seems reasonable since only a fraction of the diode radiance 
distribution (primarily the wings) sample the walls of the duct while most of the diode light reflects from the walls of the homogenizer. If we consider the full power of an entire array composed of 1600 diode bars operating at the expected $10 \mathrm{~Hz}$ with $750 \mu \mathrm{s}$ pulses with a total output peak power of $160 \mathrm{~kW}$. the cooling system for these optics should be designed to remove 7.2 watts from the duct and +2 watts from the homogenizer. 


\section{Summary}

\section{Progress}

Here we summarize our accomplishments that greatly summarize our confidence in the technical feasibility of the Mercury Laser as well as our concerns.

- Diode pump delivery has been benchmarked in terms of efficiency. heating. and measured gain in surrogate Nd:glass slabs

-Spectral sculpting concept has been developed to provide adequate beam smoothing for direct drive.

- Gas tlow in the system has been found to operate according to specifications including flow stability, vibrations. and heat removal performance

- Diode packaging issues are resolved based on using a silicon heatsink with suitable characteristics in wavelength. chirp. diode brightness. output power. and reliability. We have lost adherence to the schedule due to the abandonment of the BeO heatsink. but now we have a desirable Silicon-based technology that we intend to use

- Design of robust scalable architecture based on split backplane and nearfield placement of telescope lens. beam expansion and appropriate handling of ghosts, etalon effects, and pencil beams is complete.

- Crystal growth control of cracking, grain boundaries, anomalous absorption, and cloudiness resolved. Core is in the process of being resolved based on an increase in thermal gradients and diffusion bonding of sub-scale slabs.

- The facility is complete and operating, including the computer controls, gas handling, power supplies. an diode cooling subsystems.

\section{Value to the Laboratory}

When completed Mercury will be the highest energy/pulse diode-pumped laser ever built by an order of magnitude. It is noteworthy that the $100 \mathrm{~J}$ energy is the same as that of the Janus Laser, which is based on flashlamp-pumped Nd:glass and originally built in 1973. In addition, the Mercury Laser will be upgradable to $3 \omega$ generation, and to picosecond operation.

Use of DPSSLs offers the dimension of high repetition rate $(\sim 10 \mathrm{~Hz})$ or simply "shots-ondemand." This high rep-rated laser system could be fully exploited by employing multiple target chambers and multiplexing the laser beam between them, (and perhaps including a dedicated chamber for special materials). The high repetition rates will allow much more data averaging to reduce measurement errors, and will enable the exploration of chaotic behavior in plasmas, which is not addressable with a limited number of shots. It will also motivate the development of rep-rated targets and diagnostic capabilities. Extensive experimental studies can be executed to map out broad areas of parameter space, in contrast to the relatively limited nature of data acquired in the past. It also allows pump-probe experiments where the probe can be scanned in time to explore the detailed temporal response. This new approach could inspire a new generation of weapons research on the basis of attaining new regimes of precision and understanding.

Examples of experiments in national security that could be enhanced with kilojoule-class DPSSL target-shooters offering "cheap shots" are:

- Precise equation of state (EOS) measurements of high- $\mathrm{Z}$ materials in moderate pressure regimes (10$100 \mathrm{Mbar}$ ) of relevance to weapons physics

- Laser-plasma instability studies covering a broad range of parameter space including chaotic behavior

- Opacity of high-Z materials in "warm" temperature regimes $(0.1-1 \mathrm{keV})$

- Rep-rated $14 \mathrm{MeV}$ neutron source development for tritium production. materials testing. and science

- Target diagnostics development for NIF utilizing $x$-rays and neutrons from solid targets

- X-ray laser backlighter development and optimization to probe high-density plasmas and targets 
- Pre-staging subscale experiments prior to high-value execution on NIF

- Plasma studies such as Thompson scattering in high-pressure plasmas at high data collection rates

- Tomographic three-dimensional X-ray imaging (0.1-10 MeV) of components with high throughput. thousands of views, and small source sizes $(\sim 20 \mu \mathrm{m})$ for reconstruction of fine details

- Off-line test of laser damage and shrapnel/debris generation fro enhanced modeling of NIF-Scale performance

A major objective of the present effort relates to establishing the readiness of the Mercury DPSSL driver to proceed to the next stage and beyond to energy generation. The reliability. availability and maintainability of the laser components should be deemed to be acceptable for a future integrated research experiment or IRE ( $\mathrm{kJ}$-class laser coupled with average-power target chamber). and have a plausible means of attaining the driver requirements of inertial fusion energy (IFE) ${ }^{3 !}$. A possible program plan is sketched in Fig. 99. where efforts on NIF and fusion chamber research are included in the decision to proceed with the engineering test facility (ETF). an average-power fusion core. It is thought that addressing the IRE requirements will suggest a clear pathway for IFE DPSSL drivers for most issues. although the diode cost reduction will demand special attention. The specific long-term technology development areas can be summarized as follows:

- Increase the efficiency of the DPSSL IFE driver to between 10 to $20 \%$ by addressing improvements throughout the systerm.

- Reduce the cost of laser diode arrays to $\$ 0.50 /$ watt for the IRE and define a pathway to $\$ 0.07 /$ watt or less for IFE

- Devise and demonstrate a scheme to produce $<1 \%$ smooth irradiation on-target for direct-drive in $0.1-1$ nsec.

- Grow gain media with $>10 \mathrm{~cm}$ aperture and tabricate into laser slabs with suitable optical quality $(<$ $4 \mathrm{~nm}$ rms distortion for $0.002-2 \mathrm{~cm}$ spatial wavelengths).

- Demonstrate high-average-power frequency conversion with high efficiency (80\%) and suitable bandwidth, employing the gas cooling techniques.

- Produce beam quality of $<5 x$ diffraction-limited using active and passive wavefront correction during average-power operation.

- Demonstrate integrated performance of DPSSLs to assure engineering viability with reliable operation, including the front-end, wavefront control, gas cooling. extraction. beam smoothing, and diode arrays.

- Develop approaches for a survivable target chamber where $x$-ray yields lead to significant surface ablation and where the final optic is suitably protected from debris and $x$-rays.

- Resolve the manner in which multiple apertures and beam bundles are assembled to attain the kilojoule and megajoule level.

One possible vision of an IRE based on a $4 \mathrm{~kJ}$ DPSSL would test performance at gain-limited aperture size as well as multi-aperture bundling needed to scale to very high energy. Most of the uses described above, including the neutron source. could be accomplished with the $4 k J$ vision of an IRE.

\section{Return to the Laboratory}

Most significantly of all to LLNL. upon the successful completion of Mercury. we will be in a position to take fuller advantage of the laser technology for constructing future kilojoule-class and ultimately high repetition (shot) rate MJ-class facilities. We will then be in a financial and technological position to credibly propose the construction of rep-rated $\mathrm{kJ}$-class NIF-companion facilities by building on the technology base gleaned from our work with the Mercury laser. This system. named IRE beamlet on Fig. 103, could create a new laser facility based project over about five years with an annual budget of - $10 \mathrm{M}$ \$/year following NIF activation. A kilojoule-class system could be used for "staging" experiments prior to their implementation on the NIF. by pre-investigation some of the plasma physics issues at 
reduced scale. The next facility. named IRE in Fig. 103. could be a Nova-class $\sim 30 \mathrm{~kJ}$ diode pumped laser that could serve as a NIF companion facility for pre-staging experiments that could reasonably scaled to NIF conditions such as those currently being conducted at Nova. Since this tacility would have significantly higher repetition rate than NIF. it would allow many experiments to be thoroughly shaken down off-line prior to implementing a relatively expensive NIF shor series.

On a longer timescale. perhaps several years after first ignition on NIF. LLNL will be in a strong position to propose a follow-on laser technology to be used in a rep-rated MU facility to be constructed after the NIF. This facility. like NIF. will be a large project with initial cost estimates placing it at $\sim 2$ BS. While it may seem early to begin to think about this scenario. it is relevant to note that the NIF was actually designed and developed over a two-decade time period. If the NIF should successtully demonstrate that high fusion gain is possible in the laboratory, then the DPSSL may prove to be the route by which ICF is taken to the next level for Stockpile Stewardship and ultimately lead to an inertial fusion energy power plant in the next century that could again spawn significant new programs/projects at LLNL. Other new DOD programs would also be synergistically supported by the underlying technology developed for Mercury such as power beaming to satellites. and laser weapons for shipboard and airborne close in defense.

\section{Conclusion}

The Mercury project is developing the next-generation fusion laser at LLNL. Flashlamp-pumped $\mathrm{Nd}$ :glass systems have served as a central technology by which the physics of ICF and high energy density plasmas have been explored at the laboratory. Laser technology has progressed. however, such that it is now possible to envision systems that are not repetition-rate limited, and have much greater reliability. While this effort is primarily aimed at developing diode-pumped solid-state laser (DPSSL) technology in support of our post-NIF strategy, the abundance of near-term uses that exist strongly encourage operating of the system after the conclusion of construction. These uses include synchrotron like $x$-ray sources for $x$-ray microscopes in small laboratory environment, neutron and $x$-ray sources for NIF diagnostics development, short pulse laser development. and many other applications. This DPSSL technology would also serve as a prototypical design of an ICF driver for energy production. The time has come to begin seriously pursuing the next generation of fusion laser technology so that we are in a position to continue moving such laser technology forward for new applications in the next century. 


\section{Inertial Fusion Energy (IFE) Development Strategy:}

\section{A Look Backward from the "End Game"}

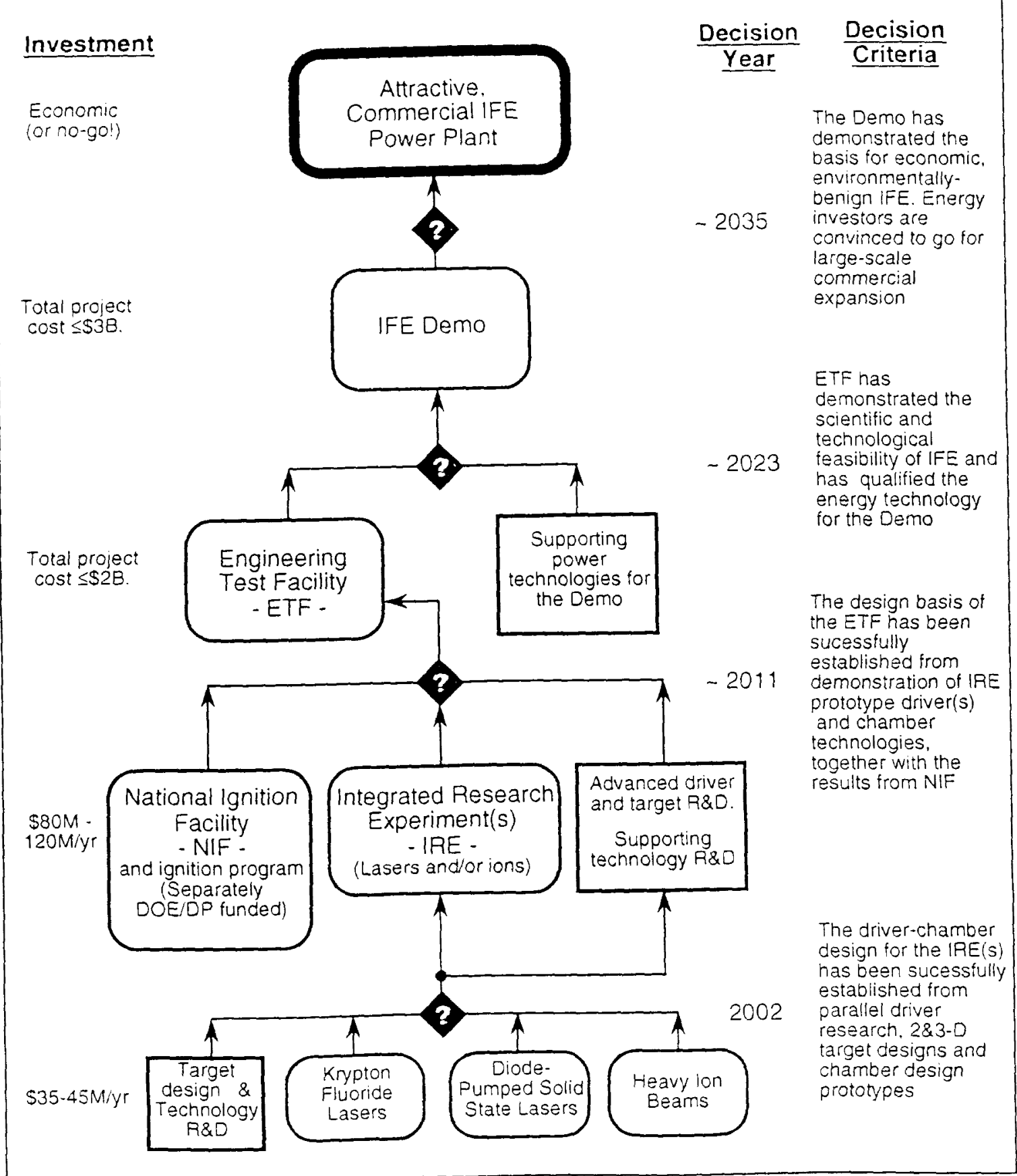

Fig. 103 IRE development strategy. 


\section{References}

W. F. Krupke. Fusion Technol. 15. 377 (1989).

2 J. L. Emmett and W. F. Krupke.. Sov. J. Quantum Electron. 13.1 (1983).

${ }^{3}$ R. J. Beach. "Theory and optimization of lens ducts". Appl. Opt.. 35. 2005 (1996).

+ S. B. Sutton. G. F. Albrecht, and H. F. Robey. "Heat Removal in a Gas Cooled Solid State Laser Disk Amplifier." AIAA Journal. vol. 30, no. 2. pp. 421-425 (1992).

${ }^{5}$ G. F. Albrecht. S. B. Sutton. H. F. Robey. and B. L. Freitas. SPIE. vol. 1040. pp. 37 (1989).

${ }^{6}$ C. P. Marshall. L. K. Smith. S. B. Sutton. M. A. Emanuel. K. J. Schaffers. S. Mills. S. A. Payne. and W.

F. Krupke. "Diode pumped gas-cooled-slab laser pertormance." OSA TOPS on Advanced Solid-State Lasers. vol. 1. pp. 208-212 (1996).

${ }^{7}$ S. B. Sutton and G. F. Albrecht. "Optimum performance considerations for a large-aperture average power solid-state laser amplifier." J. Appl. Phys.. vol. 69. no. 3. pp. 1183-1191 (1991).

"S. Sutton. "Mercury Cooling Flow Geometry - Diffuser Design." LST-ALC97-034. May 27. 1997.

"T. Woehrle and M. Eli. "Ambient Vibration Measurements of the Mercury Laser System, Bldg. 381 Rm. 1547", SM99-003TGW/MWE. (February 4. 1999).

(1) M. Eli. "Ambient Vibration Measurements of the Mercury Laser system, B-381 R-1547", SM99016MWE, (July 12. 1999).

${ }^{\prime \prime}$ C. D. Marshall, I. K. Smith, S. Sutton, M.A. Emanuel, K. I. Schaffers, S. Mills, S. A. Payne, and W. F. Krupke, "Diode-pumped gas cooled-slab laser perfromance." OSA Trends in Optics and Photonics Series, Vol. 26, pp. 635-641, (1996).

I2 L. D. DeLoach, S. A. Payne, w. L. Kway. J. B. Tassano, S. N. Dixit. and W. F. Krupke. "Vibrational structure in the emission spectra of $\mathrm{Yb}^{3+}$-doped apatite crystals," J. Luminescence, 62, 85-94 (1994).

13 R. L. Hutcheson and R. W. Equall. Scientific Materials Corporation, 310 Icepond Road, Bozeman, MT 59715 , private communication.

${ }_{14}^{14}$ B. Chalmers, Principles of Solidification (John Wiley \& Sons, Inc., New York, 1964) pp. 150-157.

${ }^{15}$ E.L. Church, "Fractal surface finish." Applied Optics, 27, 1518-1526 (1988)

${ }^{16}$ M.D. Perry, F.G. Patterson, and J. Weston, "Spectral shaping of chirped-pulse amplification," Opt. Lett. 15, p. 38I (1990).

${ }^{17}$ TEXSTAN is a variation of the STAN-5 program written by M. Crawford of the University of Texas. The reader is referred to: M. E. Crawford and W. M. Kays, "STAN-5 - A Program for Numerical Computation of Two-Dimensional Internal and External Boundary Layer Flow," NASA CR-2742, Nov. 1976.

${ }^{18}$ A. B. Shapiro, "TOPAZ3D - A three-dimensional finite element heat transfer code," LLNL, UCID-2848 , August 1985.

19 B. N. Maker, et. al., "NIKE3D - A Nonlinear, Implicit. Three-dimensional Finite Element Code for Solid and Structural Mechanics." LLNL, UCRL-MA-105268 Rev. 1, April 1995.

2) OPL, the optics code applied in this analysis is under ongoing development at LLNL by M. Rotter and S. Sutton

${ }^{21}$ S. Sutton. "Preliminary Predictions of Temperatures, Stresses, and Optical Distortions in the Mercury Laser Slabs," LST-ALC 97-058. September 1997.

22 R. A. Sacks. M. A. Henesian. S. W. Haney, J. B. Trenholme. "The PROP92 Fourier Beam Propagation Code," ICF Annual Report 1996, University of California, Lawrence Livermore National Laboratory, Report UCRL-LR-105821-96 pp.207-213 (1997).

23 J. B. Trenholme, "Damage from Pulses with Arbitrary Temporal Shapes," Lawrence Livermore National Laboratory, Livermore, CA. memo LST-LMO-94-001 (Ver. 2) L-18179-2 (1995).

${ }_{24}^{24}$ J. H. Campbell and F. Rainer. "Optical Glasses for High-Peak-Power Laser Applications." Lawrence Livermore National Laboratory, Livermore, CA. UCRL-JC-109255 (1992); and Proceedings of the 1992 SPIE Conference, SAN DIEGO. CA. July 19-24. 1992. 
${ }^{5}$ R. A. Sacks, et al., ibid.

${ }^{26}$ See for example. Chaiyu Ai and James E. Wyant. "Measurement of the inhomogeneity of a window." Optical Engineering. 30. \#9. pp. 1399-1404 (1991).

${ }^{27}$ J.K. Lawson. D.M. Aikens. R.E. English. Jr.. C.R. Wolfe. "Power spectral density specifications for high-power laser systems." SPIE Proceeding 2775-Specification. Production and Testing of Optical Components and Systems. pp. 345-356 (1996).

${ }_{2 .}$ Eugene L. Church. "Fractal Surface Finish." Applied Optics, Vol. 27. No. 8. (15 April 1988). p. 1518.

2" Physics basis for Optical Performance of the NIF amplifiers". A. Erlandson. T. Alger. S. Fulkerson. J. Horvath. K. Jancaitis. C. Marshall. M. Rotter. S. Sutton and L. Zapata. UCRL-ID-132680. NIF-001+1+2. Jan. 1999.

${ }^{13}$ A. Erlandson. T. Alger. S. Fulkerson. J. Horvath, K. Jancaitis. C. Marshall, M. Rotter. S. Sutton and L. Zapata. "Physics basis for Optical Pertormance of the NIF amplifiers". UCRL-ID-132680, NIF-001+1+2. Jan. 1999.

${ }^{31}$ C. D. Orth. S. A. Payne, and W. F. Krupke. "A diode pumped solid state laser driver for inertial fusion energy". Nuclear Fusion 36(1) 75 (1996). 
